\title{
65th ORCA Congress
}

\author{
July 4-7, 2018, Copenhagen, Denmark
}

\section{Abstracts}

\section{Contents}

Sessions 1 and 2

469 Erosion: Lab-Based Studies and De- and Re-Mineralization

Sessions 3 and 4

478 Microbiology

Sessions 5 and 6

487 Clinical Studies

Sessions 7 and 8

499 Epidemiology

Session 9

510 Fluoride and Microbiology

Session 10

519 Clinical Studies

Session 11

528 Diagnostics: Clinical Studies and Pulp-Dentinal Reactions Session 12

536 Diagnostics: Lab-Based Studies and Hard Tissues

544 Author Index 


\section{Sessions 1 and 2 Erosion: Lab-Based Studies De- and Re-Mineralization}

\begin{abstract}
1
Role of Particle Type on Toothpaste Effects on Erosion and Erosion/Abrasion in Presence of $\mathrm{NaF}$

M. Schnarr ${ }^{a *}$, N. Schlueter ${ }^{b}$, S. Rupf ${ }^{c}$, C. Ganss ${ }^{\text {a }}$

melanie.schnarr@dentist.med.uni-giessen.de aDepartment of Conservative and Preventive Dentistry, Dental Clinic, Justus-Liebig-University, Giessen, Germany; ${ }^{\text {bDivision }}$ for Cariology, Department of Operative Dentistry and Periodontology, Center for Dental Medicine, Medical Center - University of Freiburg, Faculty of Medicine, University of Freiburg, Freiburg im Breisgau, Germany; ${ }^{\mathrm{C} C l i n i c}$ of Operative Dentistry, Periodontology and Preventive Dentistry, Saarland University Medical Center, Homburg, Germany
\end{abstract}

NaF-toothpastes exhibited different effects in erosion/abrasion but also in erosion only studies. These effects are difficult to explain and may be attributed to excipients but also to the particle type. This study aimed to investigate the impact of various particle types differing in size, abrasivity and type (silica/alumina), on erosion and erosion/abrasion in the presence of NaF. Syloblanc81, Sylodent850c, PerkasilSM660, SorbosilAC77, SorbosilAC36, SorbosilAC39, and Apyral24 were investigated. Toothpastes each containing one type of these particles but no active ingredients were diluted with $\mathrm{NaF}$-solution (1400 ppm; 1:3 weight\%) to be used as slurries. In experiment 1 , enamel specimens ( $n=16$ /group) underwent cycles of de- and remineralisation ( 1 cycle: $6 \times 2$ min $0.5 \%$ citric acid, $\mathrm{pH} 2.8$, alternated by $6 \times 15 \mathrm{~min}$ remineralisation solution) and after the first/last erosion of each cycle they were immersed in slurries ( $2 \mathrm{~min}$ ). In experiment 2 , specimens were additionally brushed within immersion time (15 s, brushing machine). Controls were immersion and brushing in distilled water. Tissue loss was measured profilometrically after 3, 6, 9 and $12 \mathrm{cy}$ cles. Statistics: t-test, ANOVA, Tamhane's post hoc. In all groups and experiments, tissue loss increased significantly after 3, 6, 9 and 12 cycles ( $\mathrm{p} \leq 0.001$ each). After 12 cycles, brushing with Sylodent850c and Syloblanc81 increased values compared to slurry only $(14.7 \pm 2.5$ to $17.6 \pm 1.5 \mu \mathrm{m}$ and $16.7 \pm 3.5$ to $18.6 \pm 3.3 \mu \mathrm{m}$ resp.; $\mathrm{p} \leq 0.05$ each), all other particle types showed no significant differences between slurry only and brushing. Brushing with SorbosilAC77 caused significantly less tissue loss compared to all other particle types ( $\mathrm{p} \leq 0.05$ each), except Sylodent850c.

Interestingly, Sylodent $850 \mathrm{c}$ revealed significantly lower tissue loss compared to PerkasilSM660, SorbosilAC36 and SorbosilAC39 ( $\mathrm{p} \leq 0.05$ each) when applied as slurry only. The results emphasise the impact of the particle type on toothpaste effects both with and without brushing in presence of $\mathrm{NaF}$.

Dr. Theiss Naturwaren, Homburg, Germany, provided the experimental toothpastes as well as the particles under study. Otherwise, the study was funded by Justus-Liebig-University Giessen, Germany.

2

Interplay Between Tooth Age and Toothbrushing on Erosive Tooth Wear Susceptibility

A.A. Algarni ${ }^{\mathrm{a}, \mathrm{b}}$, F. Lippert ${ }^{\mathrm{b}}$, P. Ungar ${ }^{\mathrm{c}}$, C. González-Cabezas ${ }^{\mathrm{d}}$, J.A. Platt ${ }^{\mathrm{e}}$, G.E. Eckert ${ }^{\mathrm{f}}$, A.T. Hara*b

ahara@iu.edu

aDepartment of Restorative Dentistry, Taibah University College of Dentistry, Al Madina AI Munawarah, KSA;

${ }^{b}$ Department of Cariology, Operative Dentistry and Dental Public Health, Indiana University School of Dentistry, Indianapolis, IN, USA; ' $A$ nthropology Department, University of Arkansas, Fayetteville, AR, USA; ${ }^{d}$ Cariology Department, University of Michigan School of Dentistry, Ann Arbor, MI,

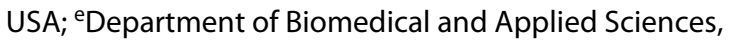
Indiana University School of Dentistry, Indianapolis, IN, USA; fDepartment of Biostatistics, Indiana University School of Medicine, Indianapolis, IN, USA

Erosive tooth wear (ETW) is a growing dental condition often associated with aging. This study investigated the effect of

* Presenting authors. 
tooth age on ETW susceptibility, under different toothbrushing-abrasion simulations. Extracted premolars had their age estimated ( 10-100 years old) using dental forensic methods [Bang and Ramm, 1970; Liversidge and Molleson, 1999]. Enamel and root dentin slabs were obtained, embedded in acrylic, flattened and polished. They were assigned into one of four toothpaste-abrasivity levels (precipitated silica) $(n=80)$ : low, medium, high or negative control (de-ionized water, DIW), following balanced randomization by age. Specimens were subjected to a daily cycling protocol consisting of 5-min erosive challenge ( $0.3 \%$ citric acid, $\mathrm{pH} 2.6)$ and 60 -min remineralization in artificial saliva (AS), 4x/day. A 15-s toothbrushing with the assigned toothpaste slurry was performed during the 1st and 4 th remineralization episodes, for $2 \mathrm{~min}$. Surface loss (SL) was measured after the 5th and 10th cycling days using optical profilometry. Effects of tooth age, toothpaste-abrasivity and time on SL were evaluated using linear mixed effects regression analysis $(\alpha=0.05)$. Increase in enamel SL with age was observed with low-abrasive and DIW groups only. Dentin SL increased with age for all treatment-time combinations. Enamel mean SL was ranked as high $>$ medium $>$ low $>$ DIW (range: $12.1-23.8 \mu \mathrm{m}$ ). For dentin, (SL range: 12.5-16.1 $\mu \mathrm{m}$ ), the low-abrasive group showed the least SL. Compared to DIW, medium (after 5 days) and high-abrasive (after 5 and 10 days) groups showed lower dentin SL at younger ages, and higher dentin SL at older ages. In conclusion, susceptibility of enamel and dentin to simulated ETW increased with age. The effect of age on enamel was more prominent when using lower abrasive systems. Simulated dentin ETW increased with age as well as toothpaste abrasive level.

This research was funded by the Oral Health Research Institute, IndianaUniversity School of Dentistry.

\section{3 \\ 3D Assessment of Erosive Tooth Wear Using Intraoral Scanner. An In Vitro Examination \\ S. Michou*a , C. Vannahme ${ }^{\mathrm{b}}$, A.R. Benetti ${ }^{\mathrm{a}}$, K.R. Ekstrand ${ }^{\mathrm{a}}$ \\ *stmi@sund.ku.dk \\ aDepartment of Odontology, Faculty of Health and Medical \\ Sciences, University of Copenhagen, Copenhagen, Denmark; \\ b3Shape A/S, Copenhagen, Copenhagen, Denmark}

This in vitro study aims to evaluate the feasibility of a 3D intraoral scanner, aided by a specific software, to detect early erosive tooth wear. Erosive tooth wear was simulated on extracted human teeth, set up in two shortened dental arches. Each dental arch (including anterior and posterior teeth) was scanned at baseline with an intraoral scanner (TRIOS ${ }^{\circledR}$, 3Shape, Denmark). The teeth were eroded with $1 \%$ citric acid solution ( $\mathrm{pH} 2.5)$ under continuous agitation (100 rpm). Every hour up to 6 hours and subsequently every 6 hours up to 24 hours, the teeth were scanned, brushed and re-scanned. The 3D models obtained from the 9 consecutive intervals were superimposed with the baseline model using a specific software (3Shape TRIOS ${ }^{\circledR}$ Patient Monitoring software, beta version, not yet commercially available). The loss of tooth substance was detected using a software tool which aligns the models relative to each other and allows measuring surface differences between the respective 3D models obtained from different time intervals. Differences above $0.05 \mathrm{~mm}$ are considered reliable in the software. Tooth substance loss was measured at 8 positions on each tooth and subsequently mean loss and standard deviation were calculated for each point in time. The software detected localized loss of tooth substance above 0.05 $\mathrm{mm}$ already after 1 hour of immersion in acid. The mean loss from baseline to 24 hours gradually increased up to $0.33 \mathrm{~mm} \pm$ $0.03 \mathrm{~mm}$. From that amount, $48 \%$ was lost during erosion while the remaining $52 \%$ was lost during subsequent toothbrushing. In conclusion, the 3D intraoral scanner system is able to detect and quantify the loss of tooth substance due to erosive tooth wear at very initial stages.

This study was funded by Innovation Fund, Denmark (Grand Solution project 13-2014-1). The authors acknowledge the Patient Monitoring Software development team at 3Shape for technical support.

\section{4 \\ Influence of NaF and SnF2 Toothpastes Abrasivity on Erosive Tooth Wear}

C.M. Assunção*a,b, N.M. Santos ${ }^{\mathrm{a}}$, N.C. Sonda ${ }^{\mathrm{a}}$, R.P. Silva ${ }^{\mathrm{a}}$, A. Lussi $^{\mathrm{b}}$, J.A. Rodrigues ${ }^{\mathrm{a}}$

crisassuncao@hotmail.com

aSchool of Dentistry, Federal University of Rio Grande do Sul, Porto Alegre, Brazil; 'b Department of Preventive, Restorative

and Pediatric Dentistry, University of Bern, Bern, Switzerland

This in vitro study investigated the effect of experimental dentifrices containing $\mathrm{NaF}$ or $\mathrm{SnF}_{2}$ fluoride with different abrasive concentration in bovine enamel wear. Ninety bovine enamel blocks $(4 \times 4 \mathrm{~mm})$ were included in epoxy resin, polished and selected by superficial microhardness (mean $369.03 \pm 70.53$ ). The specimens were submitted to 5 erosive/abrasive challenge cycles with $1 \%$ citric acid $(\mathrm{pH}=3.6), 3 \mathrm{~min}$, under agitation and brushed with electric toothbrush on an adapted device ( $2 \mathrm{~min}, 200 \mathrm{~g}$ load). The specimens were divided into different groups $(n=10)$, treated with experimental dentifrices of $1100 \mathrm{ppm}$ fluoride as $\mathrm{NaF}$ or $\mathrm{SnF}_{2}$ with different abrasivity (without abrasive silica, 10\%, 14\% and $16 \%$ abrasive silica). The placebo toothpaste had no fluoride and no abrasive silica. Superficial microhardness change $(\triangle \mathrm{SMH})$ was calculated. Data were analyzed with ANOVA, Tukey and paired $t$ tests with a significance level of $5 \% . \triangle \mathrm{SMH}$ results from different groups: NaF0\% 95.12, $\mathrm{SnF}_{2} 0 \% 178.34, \mathrm{NaF} 10 \% 127.64, \mathrm{SnF}_{2} 10 \%$ 104.4, NaF14\% 88.62, $\mathrm{SnF}_{2} 14 \%$ 85.82, NaF16\% 109.58, $\mathrm{SnF}_{2} 16 \%$ 94.72, Placebo 164.1. NaF0\% was statistically different from the placebo group $(\mathrm{p}=0.033)$ and $\mathrm{SnF}_{2} 0 \%(\mathrm{p}=0.004) . \mathrm{SnF}_{2} 0 \%$ had no statistical difference from the placebo group $(\mathrm{p}=0.999)$ and presented statistical differences from the other $\mathrm{SnF}_{2}$ groups: $\mathrm{SnF}_{2} 10 \%$ $(\mathrm{p}=0.017), \mathrm{SnF}_{2} 14 \%(\mathrm{p}=0.001)$ and $\mathrm{SnF}_{2} 16 \%(\mathrm{p}=0.004) . \mathrm{NaF}$ experimental dentifrices presented positive results in the protection against wear caused by erosive/abrasive challenge in this in vitro study. $\mathrm{SnF}_{2}$ experimental dentifrices did not show better results than the groups containing $\mathrm{NaF}$. No significant differences in $\triangle \mathrm{SMH}$ using different abrasive concentrations were found. 
The authors appreciate the support of CAPES Foundation (Ministry of Education of Brazil) for the PhD student scholarship. We are thankful to Prof A.T. Hara from, Indiana University for REA analysis.

\section{5 \\ Can the Combination of Chitosan and Hyaluronan Reduce Erosive/Erosive-Abrasive Tissue Loss in Enamel In-Vitro?}

A. Duerrschnabel*, E. Strittmatter, B. Luka, N. Schlueter

andrea.duerrschnabel@uniklinik-freiburg.de

Division for Cariology, Department of Operative Dentistry and

Periodontology, Center for Dental Medicine, Medical Center

- University of Freiburg, Faculty of Medicine, University of

Freiburg, Freiburg im Breisgau, Germany

For protection of enamel from erosion/abrasion, various mouthrinses are available. However, these preparations sometimes lead to oral sensations or mucosal burning particularly in case of xerostomia. To further increase the efficacy and, in particular, the patients' acceptance of these solutions, biopolymers could play an important role. Chitosan shows potential to protect enamel from demineralization by layer formation. For increasing efficacy, the application of different biopolymers might be an option as they theoretically could react with each other in terms of multi-layer formation. Aim of this in-vitro study was to investigate whether hyaluronan (HA) can increase the protective effect of chitosan (Ch). 256 specimens (human enamel, ground flat, polished) were divided into eight groups $(\mathrm{n}=32 \mathrm{each})$ : negative control, positive control (500 ppm F-, $\left.800 \mathrm{ppm} \mathrm{Sn}^{2+}\right)$, HAl $(50 \mu \mathrm{g} / \mathrm{ml}$, $\left.1.67 \times 10^{6} \mathrm{Da}\right), \mathrm{HA} 2\left(50 \mu \mathrm{g} / \mathrm{ml}, 1.48 \times 10^{4} \mathrm{Da}\right), \mathrm{Ch}(0.5 \%, 500 \mathrm{mPas})$, combinations of $\mathrm{Ch} / \mathrm{HA} 1, \mathrm{Ch} / \mathrm{HA} 2$ and $\mathrm{Ch} / \mathrm{HA} 1+\mathrm{HA} 2$. One half of specimens per group $(\mathrm{n}=16)$ was demineralized ( 10 days, $6 \times 2$ $\mathrm{min} /$ day, $0.5 \%$ citric acid) and exposed to solutions ( $2 \times 2 \mathrm{~min} /$ day $)$, the other half ( $\mathrm{n}=16$ per group) was additionally brushed $(2 \times 15 \mathrm{~s}$, $200 \mathrm{~g}$ ) before immersion in solutions. Substance loss was measured profilometrically (mean $\pm \mathrm{SD} ; \mu \mathrm{m})$. Under erosive conditions, Ch in combination with HA1 or HA2 could reduce tissue loss significantly ( $26 \%$ and $25 \%$ respectively) compared to the negative control $(11.8 \pm 2.2 \mu \mathrm{m}, \mathrm{p} \leq 0.05)$, whereas $\mathrm{Ch} / \mathrm{HA} 1+\mathrm{HA} 2$ had no protective effect $(11.5 \pm 1.9 \mu \mathrm{m})$. All investigated active agents could not reduce the substance loss under erosive/erosive-abrasive conditions. Even if no effect of the solutions was found under erosive/ erosive-abrasive conditions, it is quite conceivable that biopolymers could support efficacy of mouth rinses if used in combination with the standard in erosion therapy (F/Sn). Therefore, a follow-up study was performed under the same conditions with F/Sn added.

Funded by Division for Cariology, Department of Operative Dentistry and Periodontology, Center for Dental Medicine, Medical Center - University of Freiburg, Faculty of Medicine, University of Freiburg, Freiburg im Breisgau, Germany.
Effect of Hyluronan/Chitosan Additives to StannousFluoride Solutions on Erosive/Abrasive Tissue Loss in Enamel In-Vitro

\author{
B. Luka*, A. Duerrschnabel, A. Wertheimer, N. Schlueter* \\ Benedikt.Luka@uniklinik-freiburg.de \\ Division for Cariology, Department of Operative Dentistry and \\ Periodontology, Center for Dental Medicine, Medical Center \\ - University of Freiburg, Faculty of Medicine, University of \\ Freiburg, Freiburg im Breisgau, Germany
}

Chitosan can enhance the antierosive/antiabrasive effect of fluoride-stannous preparations $(\mathrm{F} / \mathrm{Sn})$ by forming protective layers on enamel and by increasing the retention of $\mathrm{Sn}$ in terms of drug delivery. Interactions between polymers with opposite charges, such as chitosan and hyaluronan, might further increase the formation of protective layers by development of multi-layers and the drug delivering effects. The aim of this study was therefore to investigate the impact of two types of hyaluronan and one chitosan on the antierosive/antiabrasive effect of F/Sn on enamel. $256 \mathrm{hu}$ man enamel specimens ( $n=32$ per group) were treated with polymer solutions and F/Sn according to a previous experiment with the difference that no F/Sn was used there: NegContr (negative control), PosContr (positive control, 500 ppm F-, $800 \mathrm{ppm} \mathrm{Sn}^{2+}$ ), HA1 $\left(50 \mu \mathrm{g} / \mathrm{ml}\right.$ Hyaluronan, $\left.1.67 \times 10^{6} \mathrm{Da}\right), \mathrm{HA} 2(50 \mu \mathrm{g} / \mathrm{ml}$ Hyaluronan, $\left.1.48 \times 10^{4} \mathrm{Da}\right)$, Ch (0.5\% Chitosan, $\left.500 \mathrm{mPas}\right), \mathrm{Ch} / \mathrm{HA} 1$, $\mathrm{Ch} / \mathrm{HA} 2$ and $\mathrm{Ch} / \mathrm{HA} 1+2$. Except for NegContr, all specimens were additionally treated with F/Sn (500 ppm F-, 800 ppm Sn ${ }^{2+}$ ). One half of the specimens ( $\mathrm{n}=16$ per group) was demineralised ( 10 days, $6 \times 2 \mathrm{~min} /$ day, $0.5 \%$ citric acid) and exposed to test solutions ( $2 \times 2 \mathrm{~min} /$ day), the other half ( $\mathrm{n}=16$ per group) was additionally brushed (2x15 s, 200g). Ch and HA were generally used separately to avoid reactions between the polymers within the solution. F/Sn was either added to the chitosan solution or used separately in groups without chitosan. Substance loss was measured profilometrically (mean $\pm S D ; \mu \mathrm{m})$. Solutions: PosContr reduced substance loss by $93 \%$ (NegContr $11.4 \pm 1.8$ ) and test solutions between $76 \%$ (Ch/HA2) and 85\% (HA2) (all p $\leq 0.001)$. Brushing: PosContr reduced substance loss by $45 \%$ (NegContr $17.9 \pm 2.6 ; \mathrm{p} \leq 0.001$ ) and test solutions between $20 \%(\mathrm{Ch} / \mathrm{HA} 1+2 ; \mathrm{p} \leq 0.05)$ and $37 \%(\mathrm{Ch} /$ HA1; $\mathrm{p} \leq 0.001)$. In conclusion, Chitosan and hyaluronan were not able to positively impact the antierosive/antiabrasive effect of F/Sn.

Funded by the Division for Cariology, Department of Operative Dentistry and Periodontology, Center for Dental Medicine, Medical Center - University of Freiburg, Faculty of Medicine, University of Freiburg, Freiburg im Breisgau, Germany. 


\section{7 \\ Dentine Erosive Wear After Brushing with Desensitizing and/or Anti-Erosive Toothpastes}

S.H. João-Souza*a,b, L.O. Sakae b, A. Lussia , T. Baumann ${ }^{\mathrm{a}}$, T. Scaramuccib ${ }^{\text {, A.C.C. Aranha }}{ }^{\mathrm{b}}$, T.S. Carvalho ${ }^{\mathrm{a}}$

samyhelen@hotmail.com

aDepartment of Preventive, Restorative and Pediatric

Dentistry, School of Dental Medicine, University of Bern, Bern,

Switzerland; 'bepartment of Restorative Dentistry, School of

Dentistry, University of São Paulo, São Paulo, Brazil

This study aimed to analyze the effect of desensitizing and/or anti-erosive toothpastes on dentine erosive wear. One hundred and sixty dentine specimens were randomly allocated into 10 groups $(n=16)$, according to the tested toothpastes, which had two main claims: desensitizing (Blend-a-Med Complete Protect Expert; Elmex Sensitive Professional; Sensodyne Rapid Relief; Sensodyne Repair and Protect); and/or anti-erosive (Candida Protect Professional; Elmex Erosion Protection; Regenerate; Sensodyne Pronamel). Artificial Saliva and Colgate Cavity Protection were used as controls. The specimens were submitted to five erosionabrasion cycles; each consisting of $60 \mathrm{~min}$ immersion in artificial saliva $\left(37^{\circ} \mathrm{C}\right), 3 \mathrm{~min}$ erosion in citric acid $\left(1 \%, \mathrm{pH} 3.6,25^{\circ} \mathrm{C}\right)$, and $25 \mathrm{~s}$ of toothbrush abrasion ( $200 \mathrm{~g}, 120$ strokes $/ \mathrm{min}, 40 \mathrm{~mm} / \mathrm{s}$ ) with a total time of $2 \mathrm{~min}$ incubation in the toothpaste slurry. Surface loss (SL, in $\mu \mathrm{m})$ was measured with an optical profilometer, and the data were analyzed with Kruskal-Wallis and Mann-Whitney tests ( $\sqrt{ }$ é $\neg \pm=0.05)$. The median and interquartile ranges for SL are presented. Lower SL values were observed for Elmex Erosion Protection (3.48; 3.32-3.89), Sensodyne Repair and Protect (3.84; $3.25-4.35)$, Artificial Saliva (4.25; 3.64-4.96), Sensodyne Pronamel (4.30; 3.75-4.51) and Elmex Sensitive Professional (4.41; 4.08$5.08)$, which did not differ from each other $(p>0.05)$. The highest SL values were observed for Regenerate $(8.17 ; 7.44-9.30)$ and Blend-a-Med Complete Protect Expert (6.40; 5.91-7.18), with no difference between them $(p>0.05)$. Elmex Erosion Protection was the only toothpaste that showed less SL than Colgate Cavity Protection $(p=0.031)$. Irrespective of their desensitizing and/or antierosive claim, the toothpastes caused different degrees of SL on dentine. No toothpaste was capable of completely protecting the dentine against erosive wear.

The authors appreciate the support of the Department of Preventive, Restorative and Pediatric Dentistry, University of Bern and the Sao Paulo Research Foundation (FAPESP, process\# 2015/23620-0).

\section{8 \\ Polyphenol-Containing Extracts from Natural Sources Have Different Effects on the Protective Properties of the Pellicle}

T. Baumann*, S. Furrer, A. Lussi, T.S. Carvalho

tommy.baumann@zmk.unibe.ch

Department of Preventive, Restorative and Pediatric Dentistry, University of Bern, Bern, Switzerland

Polyphenols interact with salivary proteins, affecting for example taste perception and the ultrastructure of the pellicle. Our aim was to investigate the effects of natural polyphenol containing extracts on the erosion protective properties of salivary pellicles. We prepared 75 enamel specimens from human molars, grinding away $200 \mu \mathrm{m}$ from the surface. They were divided into five groups $(\mathrm{n}=15)$ and initial surface microhardness $(\mathrm{SMH})$ was measured. Four different natural extracts (A-D) were purchased and solutions prepared containing $1 \mathrm{mg}$ polyphenols per $\mathrm{ml}$. The fifth group served as control (tap water). All specimens underwent five cycles consisting of pellicle formation with whole mouth saliva for $30 \mathrm{~min}\left(37^{\circ} \mathrm{C}\right)$, incubation in extract solutions or water for $30 \mathrm{~min}$ $\left(10 \mathrm{ml}, 25^{\circ} \mathrm{C}, 70 \mathrm{rpm}\right)$, another pellicle formation for $1 \mathrm{~h}$, and finally individual erosion in $10 \mathrm{ml}$ of citric acid for $1 \mathrm{~min}(1 \%, \mathrm{pH}$ 3.6, $70 \mathrm{rpm}$ ). After each cycle, $\mathrm{SMH}$ and calcium released during erosion were measured, and SMH was transformed to relative $\mathrm{SMH}$ (rSMH, in relation to initial SMH). Global differences were analyzed using a Kruskal-Wallis test, and individual differences between groups using a Mann-Whitney post-hoc test. Extract A showed the least $r S M H$ decrease $(-28 \pm 6 \%)$, followed by extract $B$ $(-41 \pm 6 \%)$ and the control $(-48 \pm 5 \%)$, with all the differences being significant $(\mathrm{p}<0.05)$. Compared to the control, which released $33.6 \mathrm{nmols} / \mathrm{mm}^{2}$ of calcium cumulatively over all cycles, extracts A $\left(18.8 \mathrm{nmols} / \mathrm{mm}^{2}\right), \mathrm{B}\left(22.7 \mathrm{nmols} / \mathrm{mm}^{2}\right)$, and C (21.1 nmols/ $\mathrm{mm}^{2}$ ) significantly reduced the cumulative amount of calcium released by $32-44 \%$ ( $p<0.01$ ), with no significant differences between them. Extract D caused erosion itself, due to its low $\mathrm{pH}$. We conclude that the polyphenol containing extracts may provide an erosion protection. This protection is likely due to a modification of the pellicle. The mechanism and nature of this modification, as well as exactly which polyphenols provide the best protection, remain to be studied further.

Funded by the University of Bern.

\section{9 \\ A Rinsing Solution Containing a Polyphenol Rich Extract and Fluoride for Erosion Protection}

\section{T.S. Carvalho, S. Furrer*, A. Lussi, T. Baumann}

tommy.baumann@zmk.unibe.ch

Department of Preventive, Restorative and Pediatric Dentistry, University of Bern, Bern, Switzerland

Polyphenols can increase the erosion protective properties of the pellicle. Our aim was to exploit this effect and develop a rinsing solution containing a polyphenol-rich extract and fluoride which 
would enhance erosion protection. We prepared enamel specimens from human molars, measured their initial surface microhardness $(\mathrm{SMH})$, and divided them into five groups $(\mathrm{n}=10)$. Five rinsing solutions were prepared or purchased: solution 1 , containing $2 \mathrm{mg} / \mathrm{ml}$ polyphenols from extract A (see abstract 8) and 500 ppm fluoride; solution 2, containing $2 \mathrm{mg} / \mathrm{ml}$ polyphenols from extract A; solution 3, containing 500 ppm fluoride; solution 4, elmex ${ }^{\circledR}$ erosion protection; solution 5 , control (tap water). All specimens underwent five cycles consisting of pellicle formation with whole mouth saliva for $30 \mathrm{~min}\left(37^{\circ} \mathrm{C}\right)$, incubation in rinsing solutions for $2 \mathrm{~min}\left(10 \mathrm{ml}, 25^{\circ} \mathrm{C}, 70 \mathrm{rpm}\right)$, another pellicle formation for $1 \mathrm{~h}$, and individual erosion in $10 \mathrm{ml}$ of citric acid for $1 \mathrm{~min}(1 \%$, $\mathrm{pH} 3.6,70 \mathrm{rpm})$. After each cycle, $\mathrm{SMH}$ was measured by performing Knoop indentations with a load of $10 \mathrm{~g}$ and a dwell time of 10 $\mathrm{s}$, and calcium release was measured by atomic absorption spectroscopy. SMH was transformed to relative SMH (rSMH) in relation to the initial SMH. Solution 1 showed the least $\mathrm{rSMH}$ decrease $(-21 \%)$, followed by solutions $4,2,3(-31 \%,-32 \%$ and $-38 \%$, respectively), and solution $5(-50 \%)$. All solutions significantly reduced final rSMH loss compared to the control ( $\mathrm{p}<0.001$ ), with solution 1 performing significantly better than all the others $(\mathrm{p}<$ 0.05 ). Solutions 1 and 4 released $22.2 \mathrm{nmols} / \mathrm{mm}^{2}$ and $16.9 \mathrm{nmols} /$ $\mathrm{mm}^{2}$ of calcium cumulatively over all cycles, which corresponded to a significant reduction ( $\mathrm{p}<0.05$ ) of $33 \%$ and $49 \%$ compared to the control ( $\left.32.9 \mathrm{nmols} / \mathrm{mm}^{2}\right)$, but they did not differ significantly from each other. In conclusion, the rinsing solution containing polyphenols and fluoride performed better than the solutions containing only one of these ingredients, and comparable to a solution known for its erosion protective properties.

Funded by the University of Bern.

\section{0 \\ Differences Between Deciduous and Permanent Enamel Demineralisation Depend on the Acid Challenge and Assessment Method}

\section{T.S. Carvalho*, B. Beyeler, A. Lussi, T. Baumann}

thiago.saads@zmk.unibe.ch

Department of Preventive, Restorative and Pediatric Dentistry, School of Dental Medicine, University of Bern, Switzerland

Previous studies have shown that deciduous teeth are more susceptible to caries-like demineralisation than permanent teeth, but studies regarding erosive demineralisation show conflicting results, probably because of the different methods used for assessments or the different strengths of acid challenges. The aim of this study was to verify the demineralisation of deciduous and permanent enamel as a function of the strength of the acid challenge. We prepared a total of 120 human enamel specimens ( 60 permanent and 60 deciduous) and divided them into groups $(n=15)$. Each group was submitted to acid challenges $(4 \times 1 \mathrm{~min} ; 10 \mathrm{ml}$ of $1 \%$ citric acid; $25^{\circ} \mathrm{C}$ ) of different strengths: $\mathrm{pH} 5$ and 3, with gentle or vigorous agitation. Relative change in surface hardness (rSMH\%) and reflection (rSRI), and the total amount of calcium (nmol) released from the enamel (CaR) were assessed. Mann-Whitney tests with Bonferroni corrections were made, and median and interquartile range (IQR) are reported. We observed significant differ- ences in the demineralisation between deciduous and permanent teeth as a function of the strength of the acid challenge. At weaker acid challenges ( $\mathrm{pH} 5 /$ gentle), differences were only observable using $\mathrm{rSMH}$, where after $4 \mathrm{~min}$ acid challenge $(\mathrm{p}=0.006) \mathrm{SMH}$ for deciduous teeth decreased 6\% (IQR: 4-7\%) and for permanent teeth 2\% (IQR: 0-3\%). Differences at stronger acid challenges ( $\mathrm{pH} 3$ /vigorous) were only detected with $\mathrm{CaR}$, where, after $4 \mathrm{~min}$ acid challenge, deciduous teeth released 35 (IQR: 32-40) nmol calcium ( $\mathrm{p}<0.001)$ and permanent teeth 26 (IQR: 24-29) nmol. No differences were observed with the rSRI. In conclusion, the differences in the demineralisation of deciduous and permanent teeth are only detectable depending on the strength of the acid challenge and the method used for the assessment of mineral loss.

Funded by the University of Bern.

11

\section{Remineralization of Early Enamel Lesions by Casein Phosphopeptide-Amorphous Calcium Phosphate}

E. Zabokova-Bilbilova*a, A. Sotirovska lvkovska ${ }^{a}$, N. Stavreva $^{\mathrm{b}}$, A. Mijoskab ${ }^{\mathrm{b}}$ V. Ambarkova ${ }^{\mathrm{a}}$

efka_zabokova@hotmail.com

aDepartment of Pediatric and Preventive Dentistry, Faculty of Dentistry,"Ss. Cyril and Methodius" University, Skopje, Republic of Macedonia; bepartment of Prosthodontics, Faculty of Dentistry,"Ss. Cyril and Methodius" University, Skopje, Republic of Macedonia

This study aimed at qualitatively evaluate the remineralization potential of casein phosphopeptide-amorphous calcium phosphate on early enamel lesions by observing treated tooth surface using scanning electron microscopy (SEM). This study was conducted on 20 subjects undergoing orthodontic treatment with premolar extraction as part of their treatment. Artificial white lesions were created with the application of $37 \%$ phosphoric acid for 20 mins. Teeth were then divided into two groups: one experimental and the other control. The customized orthodontic band with a window was bonding with the composite resin in the experimental group whereas, in the control group, the band without a window was bonding. The casein phosphopeptide-amorphous calcium phosphate (GC Tooth Mousse) paste was applied on the window region of the experimental group for 3 mins twice daily after meals for one month; no paste was applied in the control group. After 30 days, teeth were extracted carefully to avoid accidental bracket removal and stored in artificial saliva $\left(20 \mathrm{mmol} / \mathrm{l} \mathrm{NaHCO}_{3}, 3 \mathrm{mmol} / \mathrm{l}\right.$ $\mathrm{NaH}_{2} \mathrm{PO}_{4}$ and $1 \mathrm{mmol} / \mathrm{l} \mathrm{CaCl} 2$, neutral $\mathrm{pH}$ ) until analysis. The 20 premolars were prepared for SEM (JEOL JSM 5300). Determination of the calcium in enamel (40 premolars) was done by spectrophotometry. The study group lesions showed remineralization compared to the most of the teeth in the control group. Analysis of the level of calcium in enamel before and after bonding the brackets with composite resin (Dentaurum, Germany), and application of a GC Tooth Mousse clearly showed that after its application the content of calcium in enamel was significantly increased. Average value of $\mathrm{Ca}$ in the examined group of teeth is $22.38 \%$, and in the control group of teeth the average value of Ca is $20.06 \%$ ( $p<$ $0.05)$. SEM photomicrographs of the experimental groups demon- 
strated either amorphous crystals or particles scattered on the surface or lines of remineralisation along the prismatic orders. These represent the redeposited mineral phases following mobilization of calcium and phosphate from the CCP-ACP. In the control group irregularities on the enamel were observed as adherent granules or globules. These represent the redeposited mineral phases following mobilization of calcium and phosphate from the CCPACP.

In conclusion casein phosphopeptide could significantly remineralize the artificial enamel lesions. The dental cream GC Tooth Mousse could indeed be considered an effective preventive method to enhance enamel resistance against the cariogenic challenges during orthodontic therapy. Thus, topical application of GC Tooth Mousse on enamel adjacent to orthodontic accessories, incorporated as a routine clinical preventive procedure, is a simple measure of great significance. It is strongly recommended for decreasing the risk of enamel demineralization during orthodontic treatment.

\section{2 \\ Effect of High-Fluoride Dentifrice on De/ Remineralization of Root Dentine In Situ}

\author{
G.C. Vale*a, J.P. Leal ${ }^{\mathrm{a}}$, R.S. Ferreira ${ }^{\mathrm{a}}$, C.P.M. Tabchoury ${ }^{\mathrm{b}}$ \\ glauber@ufpi.edu.br

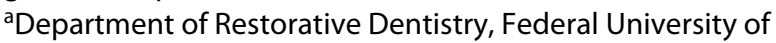 \\ Piauí, Piauí, Brazil; bPiracicaba Dental School, University of \\ Campinas, Piracicaba, Brazil
}

The aim of the present study was to evaluate the effect of highfluoride dentifrice on de and remineralization of root dentine. Ten healthy volunteers took a part on this randomized, blind, crossover and split-mouth in situ experimental study. During four phases of seven days, they wore a palatal appliance containing 4 dentine specimens ( 2 sounds and 2 in vitro demineralized) of $4 \times 4 \times 2 \mathrm{~mm}$ with initial hardness of $45.16 \pm 6.41 \mathrm{~kg} / \mathrm{mm}^{2}$ (Mean \pm $\mathrm{SD})$. The treatments were performed with silica-based dentifrice containing $0,700,1300$ and 5000 (ppm F, F as NaF). To provide a cariogenic challenge, a $20 \%$ sucrose solution was dripped three and eight times daily on the demineralized and sound specimens, respectively. After the experimental phases, the percentage of surface hardness loss (\% SHL) or recovery (\% SHR) were calculated. The statistical analysis was performed using ANOVA and Tukey test with $\mathrm{p} \leq 0.05$. The mean $( \pm \mathrm{SD})$ of $\%$ SHL and $\%$ SHR for treatments with $0 ; 700 ; 1300$ and 5000 (ppm F) dentifrices were respectively: $-66.19 \pm 7.52 \mathrm{a} ;-59.95 \pm 10.23 \mathrm{a} ;-43.19 \pm 12.62 \mathrm{~b} ;-40.71 \pm 10.25 \mathrm{~b}$; and $-15.27 \pm 6.65 \mathrm{a} ;-0.66 \pm 3.38 \mathrm{~b} ; 5.02 \pm 2.21 \mathrm{c} ; 18.00 \pm 5.32 \mathrm{~d}$. Distinct letters denote statistical difference $(\mathrm{p}<0.05)$. The results showed lower \%SHL when 5000 ppmF dentifrice was used, but without statistically significant difference from conventional dentifrice (1300 ppmF). Regarding remineralization, high-fluoride dentifrice was superior in mineral replacement compared to the others $(\mathrm{p}<0.05)$. In conclusion, high-fluoride dentifrice was effective in reducing demineralization, but its effect seems to be more important in root dentin remineralization increase.

This study was partially supported by Capes/Procad (Process num: 88881.068416/2014-01).
13

\section{Assessment of a Novel Remineralization Agents Using Optical Coherence Tomography (OCT)}

\author{
B. Sen Yavuz, B. Kargu/* \\ dtbetulsen@gmail.com \\ Department of Pediatric Dentistry, Marmara University, \\ Istanbul, Turkey
}

Previous studies have demonstrated that Optical Coherence Tomography (OCT) can be used to image the remineralization of early artificial caries lesions. OCT is able to provide sectional images of hard tissue structure, in high-resolution, being non-invasive, non-ionizing, and in real-time. The aim of study is to assessment the effect of $\mathrm{C}_{3} \mathrm{H}_{7} \mathrm{CaO}_{6} \mathrm{P}+\mathrm{MgCl}_{2}$ and $10 \%$ xylitol containing Remineralizing Gel on the remineralization of the enamel using Optical Coherence Tomography (OCT).

In our study, 20 human enamel blocks were divided into two groups as: Group-1 (R.O.C.S. Remineralizing Gel) and Group-2 (Control; Remineralization Solution contains $1.5 \mathrm{mM} \mathrm{CaCl}_{2}, 0.9 \mathrm{mM}$ $\mathrm{KH}_{2} \mathrm{PO}_{4}, 130 \mathrm{mM} \mathrm{KCl}$ and $20 \mathrm{mM}$ Hepes solution). The enamel samples were immersed in a demineralization solution maintained for 72 hours and remineralization agents were applied with $\mathrm{pH}$ cycling (ten Cate et al.,1995) for 6 days. OCT measurments was performed by OCT (1310 nm wavelength) in Anterior Segment 5 Line Raster mode at the baseline, after demineralization and after $\mathrm{pH}$ cycling. The integrated value in units was calculated in the area of peak intensity. The obtained data were analyzed statistically using ANOVA test, Kruskal Wallis test and Wilcoxen Signed Rank test with SPSS-22 program. The obtained data were evaluated at $\mathrm{p}<0.05$ significance level. A slight but significant increase in the integrated value was observed in R.O.C.S. Remineralizing Gel group. There was a decrease in the integrated reflectivity after exposure to the remineralizing gel. Images obtained from the OCT scans; the lesion depth (LD) for R.O.C.S. Remineralizing Gel $(312.7 \pm 242.7)$ was higher than control group $(203.8 \pm 111.0)$. But; no statistically significant differences were observed in the mean lesion depth between the groups ( $p>0.05)$. In conclusion, OCT images support the idea that R.O.C.S. Remineralizing Gel inhibits demineralization. These novel agents in our study can be considered effective for remineralising artificial caries lesions.

This research did not receive any specific grant from funding agencies in the public, commercial, or not-for-profit sectors.

14

\section{Comparison of the Apparent Bulk Solubility Product of Deciduous and Permanent Enamel}

\author{
L. Hassanali ${ }^{\text {a }}$, R.J. Lynch ${ }^{\text {b }}$, F.S.L. Wong ${ }^{\text {a }}$, P. Anderson*a \\ p.anderson@qmul.ac.uk \\ ${ }^{a}$ Centre for Oral Bioengineering, Institute of Dentistry, QMUL, \\ London, United Kingdom; ${ }^{b}$ Oral Healthcare, GlaxoSmithKline, \\ Weybridge, United Kingdom
}

Previous studies have suggested that deciduous enamel demineralizes more rapidly than permanent enamel, attributed to many factors including; higher carbonate content, lower mineral con- 
tent, the presence of a less mineralized neonatal line, and that deciduous enamel is substantially thinner. A fundamental contributory parameter affecting demineralization kinetics is the bulk solubility of both enamel types. A scanning microradiography (SMR) based technique has recently been published [Hassanali et al.: Front. Physiol. 2017; doi: 10.3389/fphys.2017.00714] which used SMR real-time methodology to measure rates of bulk human enamel demineralization at increasing calcium and phosphate concentrations, until the rate ceased (equilibrium), then compared with corresponding degree of saturation calculations at a range hydroxyapatite solubility values, in order to determine the apparent solubility product ( $\mathrm{pKsp}$ ) of the bulk solid. The aim was to use this SMR methodology to compare the apparent solubility product of bulk deciduous enamel, and permanent enamel, under carieslike conditions. Demineralization rates of natural surfaces of 11 human permanent and 11 deciduous enamel blocks at $\mathrm{pH}=4.0$ were measured using SMR. The temperature was maintained at $25.0 \pm 1.0^{\circ} \mathrm{C}$ throughout. $\mathrm{CaCl}_{2}$ and $\mathrm{K}_{2} \mathrm{HPO}_{4}$ were then added to the demineralization solutions yielding concentrations of $3.0 \mathrm{mM}$ calcium and $1.8 \mathrm{mM}$ phosphate $(\mathrm{Ca} / \mathrm{P} 1.67)$, and each demineralization rate re-measured. Further additions were made (maintaining $\mathrm{pH}$ ), and continued until demineralization ceased for each block. The calcium concentration required to cease demineralization for deciduous, and permanent enamel, was approximately $32.0( \pm 0.5)$ and $32.5( \pm 0.5) \mathrm{mM}$ respectively, corresponding to pKsp values of 121 for both enamel types. The similar apparent solubility products of bulk deciduous and permanent enamel contradicts previously reported differences in demineralization rates, suggesting that factors other than chemical composition contribute to the greater caries incidence reported for deciduous enamel.

Funded by GSK and BBSRC Case Award, BB/L502091/1.

\section{5}

\section{Remineralization Potential of a New Toothpaste Containing Ginger and Honey on Different Depth of Enamel Lesions}

E. Türkmen, D. Tagtekin*, F. Yanikoglu

dtagtekin@marmara.edu.tr

Department of Restorative Dentistry, Faculty of Dentistry of

Marmara University, Istanbul, Turkey

This study aimed to determine the remineralization potential of herbal toothpaste containing ginger and honey (Gumgumix ${ }^{\circledR}$, Beka-Turkey). Third molars which were extracted in the last 6 months were included in our study. The enamel samples taken from the teeth were separated into 3 groups and then immersed in the demineralization solution using shaking-incubator for different times (D1 $=18, \mathrm{D} 2=36$ and D3 = 72 hours). Different demineralization depths $(\mathrm{m}=53 \mu, \mathrm{m}=80 \mu, \mathrm{m}=140 \mu)$ were obtained respectively. The specimens were then divided into 3 subgroups $(\mathrm{R} 1, \mathrm{R} 2, \mathrm{R} 3 \mathrm{=} 35)$. Control groups was kept in artificial saliva solution; remineralization groups was brushed with toothpaste containing $\mathrm{NaF}$ and Gumgumix ${ }^{\circledR}$ at intervals. After demineralization and remineralization, all enamel samples were evaluated using light microscopy, a surface microhardness test, FluoreCam and ultrasound. Data were analyzed statistically using One way ANO-
VA. When all the demineralization depth groups are evaluated separately, the microhardness levels of the ginger-honey) applied sub-groups are significantly higher than the other groups $(\mathrm{R} 1=$ $312.06 \pm 18.80 ; \mathrm{R} 2=316.65 \pm 12.81 ; \mathrm{R} 3=260.86 \pm 7.64, \mathrm{p}<0.001$. After remineralization of the 3rd group(D3), FluoreCam size $(0.32 \pm 0.18)$, intensity $(-4.40 \pm 2.21)$ and microscope measurements $(118.4 \pm 14.15)$ were lower $(\mathrm{p}<0.05)$, microhardness levels $(260.86 \pm 7.64)$ and ultrasound $(1.03 \pm 0.15)$ were higher in the group treated with ginger-honey than in the other groups. No significant difference was found between the other variables in the groups after remineralization. In conclusion, application of herbal toothpaste containing ginger-honey showed significantly more remineralization than $\mathrm{NaF}$ in all demineralization depth groups.

This study was supported by Marmara University BAPKO (No. SAG-DUP-250416-0171).

\section{6 \\ Nano-Sized Metal Fluorides Remineralize Artificial Enamel Caries Lesions In Vitro}

\section{S. Schievelkamp ${ }^{\text {a }}$, R.J. Wierichs ${ }^{\mathrm{a}, \mathrm{b} *}$, E. Kemnitzc ${ }^{\mathrm{c}}$ J. Lauscha ${ }^{\mathrm{a}}$, M. Esteves-Oliveira ${ }^{\mathrm{a}}$, H. Meyer-Lueckeld}

rwierichs@ukaachen.de

aDepartment of Operative Dentistry, Periodontology and

Preventive Dentistry, RWTH Aachen University, Aachen,

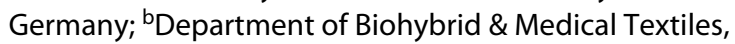
Institute of Applied Medical Engineering, RWTH Aachen University, Aachen, Germany; 'Institute of Chemistry, Humboldt University of Berlin, Berlin, Germany; ${ }^{\text {dDepartment }}$ of Restorative, Preventive and Pediatric Dentistry, University of Bern, zmk bern, Switzerland

Nano-sized materials in various forms have been proposed for remineralization of enamel and dentin in recent years. Besides small-sized calcium phosphate compounds, metal fluorides - that are synthesized via a fluorolytic sol-gel route - might bear the potential of an easily penetrating, but less dissolvable fluoride delivery source in the remineralization process of caries lesions. Thus, the aim of the study was to investigate the caries preventive effects of nano-sized metal fluorides on artificial enamel caries lesions in vitro. Bovine enamel specimens $(n=150)$ with one artificial caries lesion (median baseline mineral loss (25th/75th percentile): 9,150 $(8,631 ; 9,860)$ vol $\% \times \mu \mathrm{m})$ were prepared and randomly allocated to five groups receiving the following interventions before $\mathrm{pH}-\mathrm{cy}$ cling: application of an aqueous NaF-solution (nominal/free F-: 2,000/2,000 ppm, positive control, $[\mathrm{NaF}]$ ); ethanolic $\mathrm{CaF}_{2}$-sol (2,000/7 ppm, [CaF]); ethanolic $\mathrm{MgF}_{2}$-sol (2,000/70 ppm, [MgF]); a varnish containing $\mathrm{NaF}(22,600 / 17,700 \mathrm{ppm}$, standard control, [SC]) and no intervention (negative control, [NC]). Each sol/solution was applied five times (5x1 min) with 20 s drying in-between, whereas the varnish was applied once and removed by brushing after $48 \mathrm{~h}$. The $\mathrm{pH}$-cycling (4x60 min demineralization/day) lasted 90 days. Differences in integrated mineral loss $(\Delta \Delta \mathrm{Z})$ and lesion depth $(\Delta \mathrm{LD})$ were calculated between values after initial demineralization and after $\mathrm{pH}$-cycling using transversal microradiography. After $\mathrm{pH}$-cycling all groups showed significantly decreased $\Delta \mathrm{Z}$ values, indicating remineralization ( $\mathrm{p}<0.001$, Wilcoxon test). 
MgF and $\mathrm{CaF}[\Delta \Delta \mathrm{Z}: \mathrm{MgF}: 7,231$ (6,566; 7,491); CaF: 6,221 (5,558; $7,229)$;] showed significantly higher changes in $\Delta \Delta \mathrm{Z}$ than $\mathrm{NaF}, \mathrm{SC}$ and NC [ $\Delta \Delta \mathrm{Z}: \mathrm{NaF}: 5,190(4,050 ; 6,475)$; SC: 3,456 (2,793; 4,640); NC: 3,607 (2,927; 4,643)] ( $\mathrm{p} \leq 0.011$, Mann-Whitney test). However, no significant differences in the change of $\Delta \Delta \mathrm{Z}$ could be observed between SC and NC ( $\mathrm{p} \geq 0.786$, Mann-Whitney test). Under the condition chosen, nano-sized metal fluorides being synthesized via fluorolytic sol-gel route and showing relatively low free fluoride concentrations significantly increased remineralization of caries lesions when compared with an aqueous NaF-solution having the same nominal fluoride concentration.

This study was funded by the authors and their institutions.

\section{7}

\section{The Effect of Three Remineralizing Agents on Initial Caries Lesions - In Vitro Study}

J. Carmo*a, C. Salvado a , Q. Argi ${ }^{\mathrm{a}}$, A. Peixoto ${ }^{\mathrm{a}}$, C. Ascenso ${ }^{\mathrm{a}}$, P. Carvalho ${ }^{b}$, A.G. Manso ${ }^{\mathrm{a}}$

joanacarm@gmail.com

${ }^{a}$ CiiEM - Centro de Investigação Interdisciplinar, Instituto Universitário Egas Moniz, Egas Moniz Cooperativa de Ensino

Superior, C.R.L., Monte da Caparica, Portugal; ${ }^{b}$ CeFEMA, SINTEF

Materials and Chemistry, Oslo, Norway

The aim of the work was to analyse the effect of three remineralizing agents on initial caries lesions through scanning electron microscopy (SEM) and to compare the effect of deionized water and artificial saliva as a storage solution. 16 specimens were randomly divided into 8 groups $(\mathrm{n}=2)$. All groups were submitted to a lactic acid buffer solution ( $\mathrm{pH}=5)$ for 6 days in order to induce initial caries lesions. Groups A1; B1; C1 were then stored in deionized water and Groups A2; B2; C2 were stored in artificial saliva solution for a 7 day period, at a $37^{\circ} \mathrm{C}$ temperature. The specimens were brushed twice a day with an electric toothbrush and fluoride toothpaste (1450 ppm NaF). In Group B1-2 a high concentration calcium phosphate varnish (Clinpro ${ }^{\mathrm{TM}}$ White Varnish, 3M) was applied once, at the 7th day. In group C1-2 a CPP-ACP paste (GC Tooth Mousse, Recaldent ${ }^{\mathrm{TM}}$ ) was added daily. Group D1-2 constituted the control group i.e. no procedures were made. All specimens were analysed with SEM (JEOL JSM-700001F), a qualitative analysis. Images were compared by subjective criteria, such as interprismatic occlusion, gaps' filling and heterogeneous/homogeneous enamel surface. Images were chosen according to demineralisation and remineralisation pattern. Groups $\mathrm{A} 1 ; \mathrm{B} 1 ; \mathrm{C} 1$ : more homogenous surface images, with a greater interprismatic occlusion on $\mathrm{B} 1$ and $\mathrm{C} 1$, even greater on $\mathrm{C} 1$. Groups A2;B2;C2: more heterogeneous surface images, with an evident calcium precipitation, however there's a higher interprismatic occlusion on B2 and C2, specially on C2.

In conclusion the use of remineralizing agents showed improvements on enamel surface microscope appearance, and proved its interprismatic occlusion capacity. The combination of fluoride toothpaste with CPP-ACP produces the most homogenous enamel surface images. The storage with artificial saliva protects and helps to promote an environment much more saturated with calcium and its deposition on the enamel surface. Further studies with other techniques should be done to prove a real remineralization.

\section{8 \\ Investigating the Effect of Starch Within the Oral Environment via Constant Depth Film Fermenter}

J.M. Roberts*a, S.P. Valappil ${ }^{\mathrm{a}}$, R.J.M. Lynch ${ }^{\mathrm{a}, \mathrm{b}}$, D.J. Bradshaw ${ }^{\mathrm{b}}$, S.M. Highama

j.roberts9@liverpool.ac.uk

aHSR and School of Dentistry, University of Liverpool,

Liverpool, UK; ${ }^{b}$ GSK, Weybridge, UK

This study aimed to investigate the effect of starch within the oral environment and how this compares with the effect of sucrose using an in vitro model. Polished bovine enamel discs $(n=40)$ were placed within the dual constant depth film fermenter (CDFF) and inoculated using pooled human saliva. Both CDFFs were supplemented with artificial growth media ( $1 \mathrm{~g} / \mathrm{L}$ Lab Lemco Powder, $2 \mathrm{~g} / \mathrm{L}$ Yeast Extract, $5 \mathrm{~g} / \mathrm{L}$ Proteose Peptone, $2.5 \mathrm{~g} / \mathrm{L}$ Mucin from porcine stomach, $0.2 \mathrm{~g} / \mathrm{L} \mathrm{NaCl}, 0.2 \mathrm{~g} / \mathrm{L} \mathrm{KCl}, 0.05 \mathrm{ppm} \mathrm{F}-)$ at a constant rate $(0.38 \mathrm{ml} / \mathrm{min})$ with either $2 \%$ starch or $2 \%$ sucrose pulsed in periodically ( 30 minutes 8 times per day). 10 discs were removed on days 3, 7, 10 and 14 for bacterial analysis and enamel analysis. Bacteria samples were taken from 2 discs per pan and grown on selective agar to enumerate viable colony forming units. Enamel was analysed using quantitative light induced fluorescence (QLFD) and transverse microradiography (TMR). S. mutans growth was significantly higher when exposed to starch $(\mathrm{P} \leq 0.05)$ initially but eventually equalised $(\mathrm{P}=0.2)$, Lactobacilli and Veillonella counts were significantly higher for sucrose than starch $(\mathrm{P} \leq 0.05)$. QLF-D results show a significantly larger fluorescence loss for sucrose by days $10(\Delta \mathrm{F}=-55 \%$ vs $-33 \%, \mathrm{P} \leq 0.001)$ and $14(\Delta \mathrm{F}=-71 \%$ vs $-39 \%, \mathrm{P} \leq 0.001)$. Preliminary TMR results show from day 7 there was significantly greater mineral loss for sucrose over starch $(296 \%$. Vol. $\mu \mathrm{m}$ vs $40 \%$.Vol. $\mu \mathrm{m}, \mathrm{P} \leq 0.05)$. The results indicate that starch alone is not able to produce an environment as acidic as sucrose within the CDFF and enamel mineral loss and structure changes were significantly less with starch exposure. This may be due to limitations within the CDFF model or starch is overall less acidogenic.

This work is funded by an EPSRC/GSK studentship. 


\section{9}

\section{Quantitative Evaluation of Tooth Wear Using Autofluorescence}

S.K. Kim*a, H.S. Lee ${ }^{\text {a }}$, S.W. Park ${ }^{\text {a }}$, E.S. Lee ${ }^{\text {a }}$,

E. de Josselin de Jong ${ }^{\mathrm{a}, \mathrm{b}, \mathrm{c}}$, H.K. Kwon ${ }^{\mathrm{a}}$, B.I. Kim ${ }^{\mathrm{a}}$

drkbi@yuhs.ac

aDepartment of Preventive Dentistry and Public Oral Health, BK 21 Plus Project, Oral Science Research Institute, Yonsei University College of Dentistry, Seoul, Republic of Korea; bInspektor Research Systems BV, Amsterdam, The Netherlands; 'Department of Health Services Research, University of Liverpool, Liverpool, United Kingdom

The aim of this study was to determine the relationship of the new fluorescence parameter of Quantitative light-induced fluorescence (QLF) with the residual enamel thickness and the clinical tooth wear index (TWI). Sixty-nine permanent human molars and premolars having various occlusal worn conditions in oral cavity were collected. QLF-D images were used to determine the area of interest $(\mathrm{AOI})$ and the fluorescence parameter $\left(\Delta \mathrm{F}_{\text {wear }}\right)$ that indicate the maximum fluorescence intensity for optical quantitative evaluation of occlusal wear. Each specimen was then cross-sectioned in the buccal-lingual direction. The enamel thickness of AOI was measured using the stereomicroscope and dedicated analysis software. In addition, TWI for the initial stages of tooth wear (enamel wear stage to point of dentin exposure) was determined. Spearman's correlation analysis was conducted for determining the relationship of $\Delta \mathrm{F}_{\text {wear }}$ with enamel thickness and TWI. The $\Delta \mathrm{F}_{\text {wear }}$ values of each histological score was compared using a Mann-Whitney's U-test. A total of 76 AOI were included in the final analysis. As the enamel thickness became thinner, the $\Delta \mathrm{F}_{\text {wear }}$ values tended to be significantly higher and showed a strong correlation (Spearman's rho $=-0.825, \mathrm{P}<0.01$ ). The median $\Delta \mathrm{F}_{\text {wear }}$ values for each TWI score $1 \mathrm{a}, 1 \mathrm{~b}, 1 \mathrm{c}$ and 2 were $8.0 \%, 13.4 \%, 17.7 \%$, and $28.6 \%$, respectively. There were significant differences in the $\Delta \mathrm{F}_{\text {wear }}$ values among each of the TWI scores $(\mathrm{P}<0.01)$ and also showed a strong correlation between $\Delta \mathrm{F}_{\text {wear }}$ and TWI (Spearman's rho $=0.753, P<0.01)$. In conclusion, $\Delta F_{\text {wear }}$ values which denote the fluorescence difference using QLF showed a strong correlation with residual enamel thickness and tooth wear severity.

This study was supported by a grant from the Korea Institute of Planning and Evaluation for Technology in Food, Agriculture, Forestry and Fisheries (IPET) through High Value-added Food Technology Development Program, funded by Ministry of Agriculture, Food and Rural Affairs (MAFRA) (316071031HD020).

\section{0 \\ The Use of X-Ray Microtomography to Assess Remineralisation of Enamel by CPP-ACFP}

\author{
J.R. Fernando*, P. Shen, Y. Yuan, G.D. Walker, C. Reynolds, \\ E.C. Reynolds \\ e.reynolds@unimelb.edu.au \\ Oral Health CRC, Melbourne Dental School, The University of \\ Melbourne, Melbourne, Vic Australia
}

For decades the preferred method for assessing remineralisation of enamel has been transverse microradiography (TMR), however this method is destructive to the sample and technique sensitive. X-ray microtomography (XMT) has been increasingly used as a tool to non-destructively measure the mineral content in dental hard tissues. The objective of this study was to compare XMT and TMR analyses of enamel subsurface lesion remineralisation in vitro. Demineralised human enamel blocks had lesionhalves exposed to a $2 \%$ casein phosphopeptide stabilised amorphous calcium fluoride phosphate (CPP-ACFP) remineralisation solution at $\mathrm{pH} 5.5$ for eight days with a change of solution every two days. The enamel blocks were then assessed for percent remineralisation (\%R) using laboratory-based XMT (Bruker SkyScan 1172) (L), synchrotron-based XMT (Australian Synchrotron) (S) and TMR (T). A novel approach to segmenting lesion volumes was adopted for the XMT analyses. Statistical analysis was conducted with a repeated measure analysis of variance and Tukey comparison tests. Average $\% \mathrm{R}$ observed with $\mathrm{L}$ and $\mathrm{S}$ were found to be $20.77 \pm 7.12 \%$ and $20.55 \pm 6.27 \%$ respectively and were not significantly different $(\mathrm{p}>0.05)$. Average $\% \mathrm{R}$ observed with $\mathrm{T}$ was found to be $49.29 \pm 3.23 \%$, significantly higher than $\mathrm{L}$ and $\mathrm{S}(\mathrm{p}<$ 0.0001 ). Despite software correction, a range of artefacts including beam hardening and phase contrast were encountered in the XMT analyses that resulted in an underestimation of $\% \mathrm{R}$. It was found that TMR was more sensitive to mineral density changes in the enamel surface region and was therefore more suited than laboratory-based XMT or XMT conducted at the Australian Synchrotron for assessment of enamel remineralisation.

This study was supported by the Australian Government, Department of Industry, Innovation and Science. 


\section{Sessions 3 and 4 Microbiology}

\section{1 \\ Cariogenic and Acidogenic Potentials of Candida albicans Biofilms in Relation to Root Dentin}

C.G. de Andrade*a , G. Eidt' ${ }^{\mathrm{a}}$, T.C. Negrini ${ }^{\mathrm{a}}$, M.L. Lamers ${ }^{\mathrm{b}}$, R.A. Arthur ${ }^{\mathrm{a}}$

rodrigoarthur.ufrgs@gmail.com

aDepartment of Preventive and Community Dentistry, Federal University of Rio Grande do Sul, Porto Alegre, Brazil; ${ }^{b}$ Department of Morphological Sciences, Federal University of Rio Grande do Sul, Porto Alegre, Brazil

This study aimed to evaluate the cariogenic and acidogenic potentials of in vitro biofilms containing C. albicans associated, or not, with $S$. mutans in relation to bovine root dentin. Single-species (S. mutans ATCC 700610 - SM or C. albicans ATCC 90027 - CA) and dual-species (S. mutans + C. albicans - MIX) biofilms were grown on the surface of sound root dentin slabs with known baseline hardness during 24, 48 and 72 hours in culture supplemented with $0.12 \%$ dextrose and $0.12 \%$ sucrose, which was replaced daily. The average concentration of both SM and CA on dentin samples was approximately $0.2 \log _{10} \mathrm{CFU} / \mathrm{mm}^{2}$. The $\mathrm{pH}$ of the spent culture medium was checked every 24 hours. At each time point the percentage of surface hardness loss (\%SHL) and the percentage of structured sound remained collagen fibrils on the demineralized slabs were accessed via hardness and PicroSirius Red assays, respectively. Data were statistically analysed by two-way ANOVA $(\mathrm{p}<0.05)$. CA biofilms induced the lowest \%SHL in relation to the other biofilms, and MIX biofilms presented statistically lower \%SHL than SM biofilms, irrespective to the time of biofilm growth (CA: $-39.34 b \pm 11.53$; SM: $-80.87 \mathrm{a} \pm 7.32$; MIX: $-76.37 \mathrm{c} \pm 7.29$ ). The $\mathrm{pH}$ of spent culture medium was statistically higher in the presence of CA biofilms than SM and MIX biofilms at both 48 and $72 \mathrm{~h}$; and higher $\mathrm{pH}$ values were also found in MIX biofilms in comparison with SM biofilms (48 h-CA: 6.92b \pm 0.17 ; MIX: 4.91c \pm 0.26; SM: 4.46a \pm 0.06; 72 h-CA:6.85b \pm 0.30 ; MIX: 5.70c \pm 0.86 ; SM: $4.7 \mathrm{a} \pm 0.46)$. Remained collagen fibrils were qualitatively more organized on dentin slabs exposed to both CA and MIX biofilms. The results suggest that CA presents cariogenic potential against root dentin and the association between CA and SM reduces both the cariogenic and acidogenic potentials of SM biofilms.

This research received financial support of the Coordination for the Improvement of Higher Level Education (CAPES).

\author{
22 \\ Candida albicans and Root Caries: A Transcriptomic \\ Analysis \\ L.D. Ev*a, N. Damé-Teixeirab ${ }^{\mathrm{b}}$,T. Do ${ }^{c}$, D.A. Devine ${ }^{\mathrm{c}}$, M. Maltz ${ }^{\mathrm{a}}$, \\ D. Beighton ${ }^{\text {, C.C.F. Parolo }}{ }^{\mathrm{a}}$ \\ laisdanielaev@gmail.com \\ aFederal University of Rio Grande do Sul, Porto Alegre - Brazil; \\ bUniversity of Brasília, Brasilia - Brazil; ' University of Leeds,

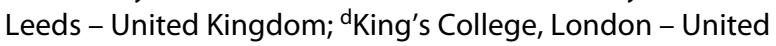 \\ Kingdom
}

Candida albicans is the most prevalent yeast found in root caries lesions. Despite that, its role in root caries pathogenesis is unknown. This study aim to observe the gene expression of C. albicans in the microbiota of root surfaces with [RC, $\mathrm{n}=9$ ] and without caries [SRS, $n=10$ ]. The differential gene expression of $C$. albicans and the specific genes related to cariogenic traits were studied in association with SRS and RC. The total bacterial RNA was extracted and the cDNA libraries were prepared and sequenced on the Illumina Hi-Seq2500. Unique reads were mapped to 162 oral microbial reference genomes (CLC Genomics workbench) including C. albicans SC5314. The putative presence of C. albicans was estimated (sum of reads/total number of genes $\geq 1$ ) in each sample. Count data were normalized using DESeq to analyse differential gene expression (DESeq2) applying the Benjamini-Hochberg correction (FDR $<0.05)$. Pearson correlation was performed to evaluate the degree and direction of correlation between C. albicans and $S$. mutans using the sum of reads assigned to species in each sample $(\mathrm{p}<0.05)$. Pearson correlation showed a significant moderate positive association $(\mathrm{r}=0.59, \mathrm{p}=0.007)$ of the putative presence of $C$. albicans and $S$. mutans. Two genes $(\mathrm{CaO} 19.610, \mathrm{FDR}=0.009$; $\mathrm{CaO} 19.2506, \mathrm{FDR}=0.018$ ) were up-regulated on SRS, and their 
functions are related to biofilm formation. Seven genes (UTP20, $\mathrm{FDR}=0.018 ;$ ITR1, FDR = 0.036; DHN6, $\mathrm{aO} 19.7197, \mathrm{CaO} 19.7838$, STT4, GUT1, FDR = 0.046) were up-regulated on RC and their functions are related to metabolic activity, sugar transport, stress tolerance, invasion and $\mathrm{pH}$ regulation. The use of alternative carbon sources, including lactate, may be an especial trait of C. albicans influencing virulence. In conclusion, C. albicans is metabolically active in biofilm of sound root surfaces and carious dentin, with different roles in health and disease.

Financial support was provided by the Brazilian National Counsel of Technological and Scientific Development (CNPQ) (process no. 482504/2013-7), the Coordination for the Improvement of Higher Level Education (CAPES) (process no 18097-120 ), and the Rio Grande do Sul State Foundation for Research Support (FAPERGS) (process no. 001/2013 PQG)and Leeds Teaching Hospitals Charitable Foundation (R\&D/PP/12011), the Dunhill Medical Trust (R245/0212).

\section{3 \\ The Cariogenicity of Core Microbiota in Early Childhood Caries}

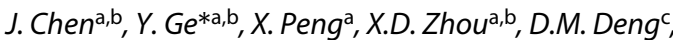 \\ L. Cheng ${ }^{\mathrm{a}, \mathrm{b}}$
}

yang_ge_dentist@hotmail.com

aState Key Laboratory of Oral Diseases, Sichuan University, Chengdu, China; ${ }^{b}$ Department of Operative Dentistry and Endodontics, West China Hospital of Stomatology, Sichuan University, Chengdu, China; 'Department of Preventive Dentistry, Academic Centre for Dentistry Amsterdam (ACTA), University of Amsterdam and Vrije Universiteit Amsterdam, Amsterdam, The Netherlands

Early childhood caries (ECC) is one of the most prevalent infectious disease in children and regarded as a consequence resulting from the disequilibrium of dental plaque ecology. Previous studies showed several species could be served as biomarkers of ECC prognosis and termed as the "core microbiota of ECC". The current study aims to verify the cariogenicity of core microbiota in comparison with Streptococcus mutans biofilms. The core microbiota (CM) biofilms comprise Fusobacterium nucleatum, Veillonella parvula, Prevotella denticola and Leptotrichia wadei. The single-species $S$. mutans biofilms with (Sm-CM) or without $\mathrm{CM}(\mathrm{Sm})$ and the $\mathrm{CM}$ biofilms alone were developed on the saliva-coated glass coverslips or enamel surfaces for $72 \mathrm{~h}$. The composition and structure of all biofilms were examined by plating and microscopy methods. The cariogenicity of the biofilms was evaluated by $\mathrm{pH}$, extracellular polysaccharide synthesis and mineral loss. The three types of biofilms were also introduced into a rat model and the modified Keyes scores were obtained after 6 weeks to indicate the severity of caries. The viable cell counts of $S$. mutans were significantly higher in SmCM biofilms ( $\log C F U 7.49 \pm 0.10$ /biofilm) than those in Sm biofilms (logCFU $6.95 \pm 0.11 /$ biofilm $)$ at $24 \mathrm{~h}(\mathrm{p}<0.05)$. Compared to Sm and CM biofilms, Sm-CM biofilms showed decreased $\mathrm{pH}$, increased total extracellular polysaccharide and mineral loss $(\mathrm{p}<$ 0.05). The rats in Sm-CM group also showed the highest Keyes scores among three groups after 6 weeks of caries-inducing diet feeding. The core microbiota itself had limited cariogenicity. However, the presence of core microbiota could promote cariogenicity of $S$. mutans biofilms and accelerate the ecologic equilibrium shifting towards the caries side. Thus, the core microbiota can be taken as a potential target for caries prevention and treatments.

This work was supported by grants from National Natural Science Foundation of China (grant number 81430011).
24
Microbial Characterization of Red Fluorescent Plaque
E.S. Lee ${ }^{* a}$, S.M. Kang ${ }^{\text {a }}$, E.de.J. de Jong ${ }^{\mathrm{a}-\mathrm{c}}$, B.I. Kim ${ }^{\mathrm{a}}$
drkbi@yuhs.ac
aDepartment of Preventive Dentistry and Public Oral Health, Yonsei University College of Dentistry, BK21 Plus Project, Republic of Korea; ' University of Liverpool, School of Dentistry, Department of Health Services Research, Liverpool, United Kingdom; 'Inspektor Research Systems BV, Amsterdam, The Netherlands

The aim of the present study was to identify the microbial characteristics of the red fluorescent plaque as detected by Quantitative light-induced fluorescence (QLF) technology using the 16S rRNA gene sequencing. Paired supragingival plaque samples were collected from each subject $(n=10)$, one was red fluorescent $(R F)$ plaque and the other was non-red fluorescent (non-RF) plaque observed by the QLF technology. Comparison of the microbial compositions between the RF plaque and non-RF plaque were performed by Illumina Sequencing analysis of the V3-to-V4 regions of the bacterial $16 \mathrm{~S}$ rRNA gene. An increase in microbial diversity was observed in the RF plaque, which had larger number of operational taxonomical units (OTUs) and had higher alpha diversity (Shannon) index values compared with the non-RF plaque. In terms of bacterial abundance and distribution based on the taxonomic composition analysis, there were significant differences in the community compositions between the two types of dental plaques. The dominant phyla of the RF plaque microbiota included Firmicutes (mean relative abundance: $24.0 \%$ ), Fusobacteria (23.6\%), and Bacteroidetes $(22.1 \%)$; those of the non-RF plaque included Firmicutes (34.5\%), Proteobacteria (30.4\%), and Actinobacteria (20.7\%). At the genus level, the 3 dominant genera of RF plaque were Prevotella (13.3\%), Leptotrichia (12.6\%), and Fusobacterium (9.3\%); those of the non-RF plaque were Streptococcus (20.5\%), Neisseria (11.6\%), and Veillonella (11.0\%). Among them, the mean relative abundance of Treponema (RF/non-RF ratio: 22.0), Selenomonas (12.3), Prevotella (9.7), and Tannerella (5.4) were significantly higher in the RF plaque as compared to the non-RF plaque ( $\mathrm{p}<$ 0.01 ), while that of the caries-related pathogens, such as Streptococcus (RF/non-RF ratio: 0.4 ) and Actinomyces (0.2), were higher in the non-RF plaque. In conclusion, RF emitted from the dental plaque is associated with changes in the microbial compositions and enrichment towards the periodontopathic pathogens.

This research was supported by Basic Science Research Program through the National Research Foundation of Korea (NRF) funded by the Ministry of Education under grant (No.2016R1D1A1A09916934). 


\section{5}

\section{Photodynamic Inactivation Activities of Chlorine e6 and Methylene Blue on Streptococcus mutans Biofilms}

Min Nie*a,b Dong Mei Deng ${ }^{\mathrm{b}}$, Yafei Wu', Kléber Thiago de Oliveirac', Vanderlei Salvador Bagnato ${ }^{\text {, }}$ Alessandra Nara de Souza Rastelli $\mathrm{e}^{\mathrm{C}}$

minniezou@hotmail.com

aState Key Laboratory of Oral Diseases, West China Hospital of Stomatology, Sichuan University, Chengdu, China; ${ }^{b}$ Department of Preventive Dentistry, Academic Centre for Dentistry Amsterdam, University of Amsterdam and Vrije Universiteit Amsterdam, Amsterdam, Netherlands; ${ }^{C}$ Federal University of São Carlos - UFSCAr, Department of Chemistry, São Paulo, Brazil; dUniversity of São Paulo - USP, Physics Institute of São Carlos - IFSC, São Paulo, Brazil; 'University of São Paulo State - UNESP, Araraquara, School of Dentistry, Department of Restorative Dentistry, São Paulo, Brazil

With the emergence of antibiotic resistance, there is a demand for alternative therapies, such as photodynamic inactivation (PDI), for the treatment of oral diseases. Recent photosensitizer developments have greatly improved the antimicrobial efficacy of PDI. The aim was to compare the killing efficacy of the second generation photosensitizer Chlorine e6 (Ce6) with the most commonly used photosensitizer, Methylene Blue (MB) on Streptococcus mutans UA159 biofilms. The biofilms were grown in an active attachment model in the presence of $0.2 \%$ sucrose. The $48 \mathrm{~h}$ biofilms were incubated with $0,25,50,100$ and $200 \mu \mathrm{M}$ of Ce 6 or MB for 5 min and irradiated with LED light source $\left(660 \mathrm{~nm}, 19 \mathrm{~mW} / \mathrm{cm}^{2}\right.$, $15 \mathrm{~J} / \mathrm{cm}^{2}$ ) for $13.25 \mathrm{~min}$. The efficacy of the treatments were evaluated by colony forming units (CFUs) and biofilm lactic acid production. Reactive oxygen species (ROS) production of MB and Ce6 after irradiation were measured using SOSG and HPF fluorescence probes. The lactic acid production of biofilms were already significantly inhibited at $25 \mu \mathrm{M}$ of Ce6 and MB. A dose dependent reduction in CFU counts was observed after the application of Ce6 with irradiation. Ce6 at $200 \mu \mathrm{M}$ with irradiation resulted in $5.1 \pm$ $0.9 \log$ reduction in biofilm viability. However, MB at $25 \mu \mathrm{M}$ with irradiation resulted in $1.8 \pm 0.5 \log$ reduction in biofilm viability, this killing efficacy did not increase with increasing concentrations of MB. The ROS measurements indicated that $\mathrm{Ce} 6$ and MB generated both type I and type II radicals. An optimum concentration of Ce6 or MB was required to achieve the highest ROS production. In conclusion, at high concentration, $\mathrm{Ce} 6$ is more effective for biofilm control than MB. The efficacy of MB cannot be improved by raising its concentrations.

\section{6}

Antibiofilm Properties of Sodium Bicarbonate and Highly Pure Chlorine Dioxide

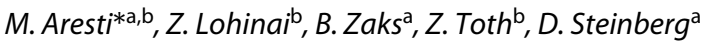 \\ mikaela.aresti92@gmail.com \\ aBiofilm Research Laboratory, Institute of Dental Sciences, \\ Faculty of Dentistry, Hebrew University, Hadassah, Jerusalem, \\ Israel; ${ }^{b}$ Department of Conservative Dentistry, Semmelweis \\ University, Budapest, Hungary
}

The control of biofilm growth is essential for the maintenance of good dental health. The aim of this research was to examine the antibiofilm properties of non-toxic substances as sodium bicarbonate $\left(\mathrm{NaHCO}_{3}\right)$ and newly developed highly pure chlorine dioxide $\left(\mathrm{ClO}_{2}\right)$ on Streptococcus mutans. S. mutans biofilm formation and preformed biofilm destruction were examined after exposure of different concentrations of $\mathrm{NaHCO}_{3}$ and highly pure $\mathrm{ClO}_{2}$ (Solumium) with crystal violet staining (total biomass analysis), MTT assays (metabolic activity) and confocal laser scanning microscopy (ratio of dead/live bacteria in different biofilm depth) and analysed by ANOVA. Our results revealed significant inhibition of $S$. mutans biofilm formation with $\mathrm{NaHCO}_{3}$ or more powerful with $\mathrm{ClO}_{2}$ in comparison with biofilm formation alone. NaH$\mathrm{CO}_{3}$ displayed much less demolition on preformed biofilm than $\mathrm{ClO}_{2}$. These observations mirrored the differences found in investigation of their minimal inhibitory concentration. Our data demonstrate that both $\mathrm{NaHCO}_{3}$ and highly pure $\mathrm{ClO}_{2}$ are powerful inhibitors of biofilm development, but show less effects on the biofilm destruction. Clinical application of these agents is suggested as effective non-toxic biocides for the prevention of dental biofilm formation and therefore the consequent tooth decay.

This study was funded in part by the Erasmus+ program and Hungarian Scientific Research Fund Grants K112364.

\section{7 \\ Candida albicans Increases Dentin Demineralization Provoked by Streptococcus mutans Biofilm

\author{
A.A. Del Bel Cury*, A.A. Sampaio, S.E. Souza, A.P. Ricomini-Filho, \\ J.A. Cury \\ delbelcury@gmail.com \\ Piracicaba Dental School, University of Campinas, Piracicaba, \\ Brazil
}

Streptococcus mutans are considered one of the most acidogenic bacteria, but it has been suggested that Candida albicans could augment its cariogenicity. However, the effect of this combination of microorganisms on dentin caries has not been experimentally evaluated. Biofilms of C. albicans ATCC 90028, S. mutans UA 159 and C. albicans + S. mutans $(\mathrm{n}=12 /$ biofilm) were grown in UTYEB medium for $96 \mathrm{~h}$ on root dentine slabs of known surface hardness ( $\mathrm{SH}$ ) and exposed $8 \mathrm{x} /$ day for $3 \mathrm{~min}$ to $10 \%$ sucrose. The medium was changed $2 \mathrm{x} /$ day, after the 8 cariogenic challenges and after the night period of famine, and aliquots were analyzed to determinate the $\mathrm{pH}$ (indicator of biofilm acidogenicity). After $96 \mathrm{~h}$, 
biofilms were collected to determine wet weight, colony forming units (CFU), and amounts of extracellular polysaccharides (soluble and insoluble). Dentine demineralization was assessed by surface hardness loss (\%SHL). The architecture of the biofilms was analyzed by confocal laser scanning (CLSM) and transmission electron microscopies (TEM). Data were analyzed by ANOVA followed by Tukey's test $(\alpha=0.05)$. The two-species $C$. albicans $+S$. mutans biofilm provoked higher \%SHL on dentine $(\mathrm{p}<0.05)$ than S. mutans and C.albicans biofilm (\%SHL $=48.8 \pm 8.5 ; 36.2 \pm 10.9$; $2.8 \pm 3.4$, respectively). This was supported by the results of biofilm acidogenicity and amounts of soluble extracellular polysaccharides $(6.4 \pm 2.14 ; 4.0 \pm 0.94 ; 1.9 \pm 0.97$, respectively) and insoluble $(24.9 \pm 9.2 ; 18.9 \pm 5.9 ; 0.7 \pm 0.5)(\mathrm{p}<0.05)$. C. albicans biofilm exhibited low cariogenicity. The images of CLSM and TEM, respectively, suggested that the $C$. albicans $+S$. mutans biofilm is more voluminous than $S$. mutans biofilm and that $S$. mutans cells interact with $C$. albicans throughout polysaccharides from the biofilm matrix. The findings show that C. albicans enhances the cariogenic potential of $S$. mutans biofilm, increasing root dentine demineralization. $5)$.

Funded by Funcamp (Proc. 4887-1); CNPq (Proc.165331/2013-

\section{8 \\ Candida albicans in Intact In Vivo Biofilm from Caries Lesions}

I. Dige*, B. Nyvad

idige@dent.au.dk

Department of Dentistry and Oral Health, Aarhus University, Aarhus, Denmark

There is conflicting evidence on the association between oral candida and caries, and information about the colonization pattern of these species in natural dental biofilms is lacking. This study investigated the spatial localization of Candida albicans in intact in vivo biofilm from caries lesions in root and occlusal surfaces. Biofilm from 8 sites from root caries surfaces in 6 extracted teeth and 4 sites from occlusal caries surfaces in 4 teeth were analyzed. The occlusal sites were selected from a previous FISH study of 11 teeth [Dige et al.: Caries Res 2014;48:451-460] showing presence of possible yeast-like organisms. The teeth were fixed (paraformaldehyde 3\%), embedded (Technovit 8100 ), sectioned and decalcified (EDTA 17\%, pH 7.0) before Fluorescence in situ hybridization [Dige et al., 2014] using oligonucleotide probes against all bacteria (EUB338), all yeast (PF2), streptococci (STR405, MUT590) and C. albicans (CAAL). Sections were analyzed using a fluorescence microscope. Yeasts, most of which were C. albicans, colonized about half of the caries lesions. Two characteristic colonization patterns were apparent: In one pattern, the biofilm presented defined areas comprising Candida hyphal networks mixed with bacteria including streptococci, often extending through the entire biofilm thickness. In the other pattern, Candida formed corncob configurations with streptococci in the biofilm surface layer. Mutans streptococci formed isolated colonies and did not typically appear in close proximity with Candida. Our observations support that Candida species are regular members of the caries microbiota. For the first time, we showed that $C$. albicans populates caries biofilm in mixed colonies with bacteria. Co-localization of Candida and non-mutans streptococci indicates a beneficial interaction between these organisms. Further studies should reveal the nature of such ecological phenomena.

We gratefully acknowledge the financial support from the Danish Dental Association and The Espen Leth-Espensens and Mehlsens Foundation.

\section{9 \\ Oral Microbiome Development During Childhood: an Ecological Succession Influenced by Postnatal Factors and Associated with Tooth Decay}

M. Dzidic a , T. Abrahamsson ${ }^{\mathrm{b}}$, A. Artacho a , M.C. Colladoc, M. Stensson ${ }^{\mathrm{d}}$, M.C. Jenmalm ${ }^{\mathrm{e}}$, A. Mira*a

mira_ale@gva.es

aDepartment of Health and Genomics, Center for Advanced Research in Public Health, FISABIO, Valencia, Spain; ${ }^{b}$ Department of Clinical and Experimental Medicine, Division of Autoimmunity and Immune Regulation, Linköping University, Linköping, Sweden; 'Institute of Agrochemistry and Food Technology (IATA-CSIC), Valencia, Spain; ${ }^{\mathrm{d} C e n t r e}$ for Oral Health, School of Health and Welfare, Jönköping University,

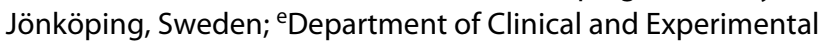
Medicine, Division of Pediatrics, Linköping University, Linköping, Sweden

Information on how the oral microbiome develops during early childhood and how external factors influence this process is scarce. In the current study, we used high-throughput DNA sequencing of the $16 \mathrm{~S}$ rDNA gene to characterise bacterial composition and diversity in saliva samples collected at 3, 6, 12, 24 months and 7 years of age in 90 longitudinally followed children, for whom clinical, dietary and health data were collected. Bacterial diversity and richness increased steadily from birth throughout childhood, ranging from an average of 392 species at 3 months of age to 452 by 7 years. Bacterial composition patterns changed through time, starting with "early colonizers" associated with the initial breastfeeding period, including Streptococcus and Veillonella; other bacterial genera such as Neisseria and Actinomyces settled after one or two years of age. Dental caries development at 9 years of age was associated with diverging microbial composition through time, especially after one year of age (CCA Anaysis, Adonis p-value = 0.0018 ). Oral pathogens like $S$. mutans or $P$. gingivalis were found to be present already in 3-month samples, before teeth eruption, at proportions of 0.01 and $0.25 \%$, respectively. Streptococcus cristatus appeared to be associated with increased risk of developing tooth decay (LefSe Analysis, $p=0.026$ at 3 months of age and $p=0.033$ at 24 months) and its role as an etiological factor and as potential biomarker of the disease should be further studied. Infants born by $\mathrm{C}$-section had initially skewed bacterial content compared to vaginally delivered infants (CCA Anaysis, Adonis p-value $=0.0016$ ), but this was recovered with age. Shorter breastfeeding habits and antibiotic treatment during the first 2 years of age were both associated with a distinct bacterial composition at later age. Taken to- 
gether, the data are consistent with oral microbiota development being an ecological succession. Thus, an altered colonization pattern during the first year of life may have long-term consequences for oral microbiome development, whose potential impact on the child's oral and systemic health should be further studied.

Funding: A.M.: Spanish Ministry of Economy andCompetitiveness (grant no.BIO2015-68711-R). M. S.: The ResearchCouncil for the South-East Sweden (grantno: 79001). M. C. J.: The SwedishResearch Council (2016-01698); the Swedish Heart and Lung Foundation (20140321); the Medical Research Councilof Southeast Sweden (FORSS-573471); theCancer and Allergy Foundation. M. C. C.: European Research Council (ERC-startinggrant 639226).

\section{0 \\ In Situ Shift of the Supragingival Microbiota in Response to Frequent Sucrose Consumption}

\section{A.C. Anderson*a, M. Rothballer ${ }^{\mathrm{b}}$, M.J. Altenburger ${ }^{\mathrm{a}}$, L. Karygiannic, J.P. Woelber ${ }^{\text {a }}$ E. Hellwig ${ }^{\text {a }}$, M. Schmid ${ }^{\text {, }}$, A. Al-Ahmad ${ }^{\mathrm{a}}$}

annette.anderson@uniklinik-freiburg.de aDepartment of Operative Dentistry and Periodontology, Faculty of Medicine, Albert-Ludwigs-University Freiburg,

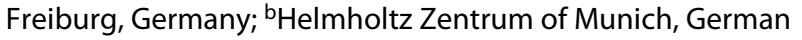
Research Center for Environmental Health, Research Unit Microbe-Plant Interactions, Neuherberg, Germany; ${ }^{C} \mathrm{Clinic}$ of Preventive Dentistry, Periodontology and Cariology, Center of Dental medicine, University of Zurich, Zurich, Switzerland

Although caries development is associated with a shift in microbial communities of dental biofilms and driven by frequent sucrose consumption, the detailed etiology is not fully understood. A diverse spectrum of acidogenic species has been linked with caries, yet to date little data is available on environmentally induced shifts in plaque in vivo. The study aimed to investigate the direct influence of a dietary change on the supragingival microbiota. Splint systems containing bovine enamel slabs were worn by eleven adults in two phases for $3 \times 7$ days with 7 -day intermissions to obtain supragingival biofilm. In phase I the adults kept their regular diet while in phase II they sucked $10 \mathrm{~g}$ rock candy per day for 3 months. The biofilm was collected during five weeks at the end of this phase. The diet of the participants was monitored. Highthroughput DNA sequencing using Illumina MiSeq of variable regions v1-v2 of the $16 \mathrm{~S}$ rDNA was applied to analyze the microbial community. Sequences of the genus Streptococcus were manually analyzed to the species level using ARB software. In addition, the surface roughness of the bovine enamel was measured with a $3 \mathrm{D}$ Laserscanning microscope. The sequencing achieved an average of 50,000 reads per sample. The results showed a significant difference in the beta-diversity and a significantly decreased species richness in phase II. Individual microbiota were dominated by Firmicutes $(58.01 \%)$, streptococci in particular (37.98\%) and the sucrose phase was marked by a significant increase of the relative abundance of streptococci, specifically $S$. gordonii $(\mathrm{p} \leq 0.001 ; \mathrm{PI}=$ $1.66 \%$, PII $=4.27 \%), S$. parasanguinis $(\mathrm{p}=0.04 ; \mathrm{PI}=1.27 \%, \mathrm{PII}=$ $2.02 \%)$ and $S$. sanguinis ( $\mathrm{p}=0.01 ; \mathrm{PI}=2.84 \%, \mathrm{PII}=4.64 \%)$ in most individuals. The roughness of the enamel surface began to increase in phase II. The results showed an increased prevalence of nonmutans streptococci owing to increased consumption of sucrose, hence supporting the extended ecological plaque hypothesis.

This study was supported by the GermanResearch Foundation (DFG, AL-1179/2-1).

\section{1 \\ Expression of Lactate Dehydrogenase in Lactobacillus spp. from Root Caries}

\author{
C.C.F. Parolo*a, N. Damé-Teixeirab , A.C. Cardoso ${ }^{\mathrm{a}}$, T. Doc, \\ D.A. Devine ${ }^{\mathrm{c}}$, D. Beighton ${ }^{\mathrm{d}}$, M. Maltz $^{\mathrm{a}}$ \\ fatturiparolo@yahoo.com \\ ${ }^{a} F e d e r a l$ University of Rio Grande do Sul, Porto Alegre - Brazil; \\ bUniversity of Brasília, Brasilia - Brazil; ' University of Leeds,

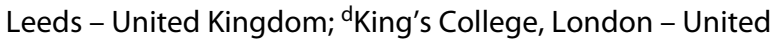 \\ Kingdom
}

The ability of lactobacilli to ferment a variety of carbohydrates and to survive in a low $\mathrm{pH}$ environment is the major virulence trait related to dental caries progression. Lactobacillus species present a constitutive gene lactate dehydrogenase (ldh), responsible for the conversion pyruvic acid into lactic acid, one of the responsible for the $\mathrm{pH}$ drop in the cariogenic biofilm. The objective of this study was to observe the gene expression of ldh genes in lactobacilli found in root caries ( $\mathrm{RC}, \mathrm{n}=30$ patients, $\mathrm{n}=9$ libraries). The total bacterial RNA was extracted and the cDNA libraries were prepared and sequenced on the Illumina Hi-Seq2500. Unique sequence reads were mapped to 162 oral microbial reference genomes including 20 species of Lactobacillus spp (CLC Genomics workbench). The putative presence of Lactobacillus was estimated (sum of reads/total number of genes $\geq 1$ ) in each sample. The genes (EC Number 1.1.1.27 and EC Number 1.1.1.28), that code for ldh, were located in the Data bank for each studied species. The percentage of the ldh expression was calculated as a proportion of all reads by species transcriptome. The ldh expression was variable in the studied samples. Higher values of ldh expression were found in some samples of L. amylolyticus (mean $2 \%$, range $0.00 \%-8.27 \%$ ), L. salivarius (mean $0.97 \%$, range $0.23 \%-3.45 \%$ ), L. johnsonii (mean $0.66 \%$, range $0.07 \%-2.39 \%)$ and $L$. jensenii $(0.80 \%$, range $0.05 \%-$ $2.31 \%)$. Ldh expression values lower than $1 \%$ were predominant in most species. In the comparison of ldh expression, a difference between the studied species was found, being lower in L. gastricus $(p=0.017)$. The fact that the ldh genes are expressed shows that acid production is active and relevant. Metabolic active Lactobacillus spp. from root caries play an important role in the biofilm virulence by the ldh expression and, consequently, acid production.

Financial support was provided by the Brazilian National Counsel of Technological and Scientific Development (CNPQ) (process no. 482504/2013-7), the Coordination for the Improvement of Higher Level Education (CAPES) (process no 18097-120 ), and the Rio Grande do Sul State Foundation for Research Support (FAPERGS) (process no. 001/2013 PQG) and Leeds Teaching Hospitals Charitable Foundation (R\&D/PP/12011), the Dunhill Medical Trust (R245/0212). 


\section{2}

\section{Gene Expression Profile of Scardovia spp. and Comparison to Other Cariogenic Bacteria in Root Caries}

N. Dame-Teixeira*a, C.C.F. Parolob, M. Maltz ${ }^{\text {b }}$ D.A. Devine ${ }^{c}$, T. Do ${ }^{c}$ nailedame@hotmail.com

aDepartment of Dentistry, University of Brasília, Brasília, Brazil;

${ }^{b}$ Department of Social and Preventive Dentistry, Federal

University of Rio Grande do Sul, Porto Alegre, Brazil; 'Division of Oral Biology, School of Dentistry, University of Leeds, Leeds, UK

Scardovia spp. has been identified as a predominant member of caries lesion biofilms; however, little is known regarding its involvement in caries pathogenesis. The purpose of this study is to assess the gene expression profile of Scardovia spp. in root caries, and compare it with that of S. mutans and Lactobacillus spp., which are well-established cariogenic microorganisms. Clinical samples from active root caries lesions were collected. Total microbial RNA was isolated and cDNA sequenced on the Illumina Hi-Seq2500 ( $\mathrm{n}=30$ patients; $\mathrm{n}=9$ libraries). The function and composition of the metabolically active microbiota were investigated using two methods: a) reads mapping to 162 oral bacterial reference genomes (CLC Genomics workbench), and b) denovo assembly of the read data and mapping to contigs and annotation of the contigs using the ncbi nr protein database with diamond software. Normalization was performed and the relative proportion of species was calculated (sum of unique reads/all reads of the sample*100). Pearson correlation was performed to evaluate the degree and the direction of correlation between Scardovia spp., S. mutans and Lactobacillus spp. $(\mathrm{p}<0.05)$. Results showed a proportion of S. inopinata and $S$. wiggisae ranging from $0.00-10.42 \%$ and $0.00-33.61 \%$ in root caries, respectively. There is a positive correlation between the transcriptome of Lactobacillus spp. and Scardovia spp. (analysis a = $0.97 ; \mathrm{p}=6.265 \mathrm{e}-06$; analysis $\mathrm{b}=0.92 ; \mathrm{p}=0.0004)$. Analysis of the carbohydrate pathways suggests the utilization of the "Bifid shunt" by $\mathrm{S}$. inopinata in root caries. The key enzyme for this metabolic pathway is expressed, fructose 6-phosphate phosphoketolase (Median $=0.83 \% ; 25$ th -75 th $=0.2 \%-1.3 \%$ ). This pathway allows the production of more energy from carbohydrates compared to that produced by the Embden-Meyerhof-Parnas pathway. Many genes coding $\mathrm{ABC}$ transporters have high levels of expression in both species. In conclusion, there is a correlation between the presence of Lactobacillus spp. and Scardovia spp. in root caries. It could suggest a symbiotic relation between these species. Bifid shunt and ABC transporters are involved in carbohydrate metabolism.

Financial support was provided by Leeds Teaching Hospitals Charitable Foundation (R\&D/PP/12011), the Dunhill Medical Trust (R245/0212), the Brazilian National Counsel of Technological and Scientific Development (CNPQ) (process no. 482504/20137 ), the Coordination for the Improvement of Higher Level Education (CAPES) (process no 18097-12-0), and the Rio Grande do Sul State Foundation for Research Support (FAPERGS) (process no. 001/2013 PQG).

\section{3}

\section{Temporal Development of the Saliva Microbiome in} Infants

S.G. Dashper*a , H.L. Mitchell ${ }^{\mathrm{a}}$, K.-A. Le Cao $^{\mathrm{b}}$, P. Solange ${ }^{\mathrm{b}}$,

L. Carpenter ${ }^{\mathrm{c}}$, M. Gussy ${ }^{\mathrm{d}}$, D. Catmulla ${ }^{\mathrm{a}}$, E. Waters ${ }^{\mathrm{c}}$, E.C. Reynolds ${ }^{\mathrm{a}}$

stuartgd@unimelb.edu.au

${ }^{a}$ Oral Health CRC, Melbourne Dental School, The University of Melbourne, Australia; belbourne Integrative Genomics, The University of Melbourne, Australia; 'School of Population and Global Health, The University of Melbourne; ${ }^{\text {R Rural Health }}$ School, La Trobe University; Australia

Early Childhood Caries (ECC) is major public health problem affecting well over $50 \%$ of Australian children. Saliva is an easily obtained secretion that contains oral bacterial species that can potentially act as biomarkers for the bacterial dysbiosis in supragingival plaque that leads to the clinical manifestation of early childhood caries. The aim of this study was to characterise the temporal development of the saliva microbiome over the first four years of life. We characterised the saliva microbiome of 143 children enrolled in a cohort study at six time points from the age of two months until four years of age. Next-Generation sequencing based community profiling targetting the $\mathrm{V} 4$ region of the $16 \mathrm{~S}$ RNA genes of the bacteria was used to analyse and quantify the microbial communities in saliva. The sequenced saliva samples generated 33 million 16S DNA sequences, on average $\sim 35,000$ sequences were identified per saliva sample with 356 operational taxonomic units at a species level identified. Over time there was an increase in the number of bacterial taxa at a species level in the saliva of infants from a mean of 31 at 2 months of age to 72 at 18 months and then a slower increase to 85 at 48 months of age. Principal component analysis of the temporal development of the saliva microbiome showed a distinct shift in the composition of the microbiome over time. Increased abundances of Streptococcus mutans and TM7 (Candidatus Saccharibacteria) in saliva at 18 months of age were predictive of active disease whilst the abundance of $\mathrm{Mo}$ raxella caprae was associated with health. In conclusion, the saliva microbiome of infants contains biomarker species that may predict the onset of early childhood caries.

Funded by Australian National Health and Medical Research Council; Oral Health Cooperative Research Centre. 


\section{4}

\section{Quantitative Analysis and Gene Expression of Lactobacillus casei Group in Children with Early Childhood Caries}

\author{
A.C. Martins Reis ${ }^{\mathrm{a}}$, D.S. Bezerra ${ }^{\mathrm{b}}$, B.G. Neves ${ }^{\mathrm{b}}$, S.F.F. Guedes ${ }^{\mathrm{b}}$, \\ E. Harth-Chúc, L.K.A. Rodrigues*a,b \\ lidianykarla@ufc.br \\ aPostgraduate Program in Medical Microbiology, Federal \\ University of Ceará, Fortaleza, Brazil; bPostgrauate Program \\ in Dentistry, Federal University of Ceará, Fortaleza, Brazil; \\ 'Piracicaba Dental School, UNICAMP, Piracicaba, SP, Brazil
}

Lactobacillus casei, Lactobacillus paracasei, and Lactobacillus rhamnosus form a closely related taxonomic group (Lactobacillus casei group) and are strongly associated in caries progress, especially in dentin. This study aimed to detect and quantify L. casei group, L. casei/paracasei and L. rhamnosus and the expression of selected L. casei group genes in active and arrested dentin carious lesions of children with early childhood caries (ECC). Dentine samples (25 active and 13 arrested carious lesions) were collected from pre-school children aged 2-5 years. Dentinal-cavitated lesions were evaluated by the Nyvad criteria. Total RNA was extracted from the dentine samples, converted to CDNA and the reverse transcription quantitative real-time PCR (RT-qPCR) analyses were performed for the bacterial quantification and for analyses of genes expression associated with survival $(\mathrm{spxB})$ and virulence (spaC, spaE and wzB). Data were analyzed with BioEstat 5.3 program. The Student t-test and Mann-Whitney test were used. Our findings revealed that species of the $L$. casei group are part of the viable microbial community in dentin caries lesions, being $L$. case $i$ group $(\mathrm{p}=0.004)$, L. casei/paracasei $(\mathrm{p}=0.001)$, L. rhamnosus $(\mathrm{p}=$ 0.022 ) more abundant in active dentin lesions. Data related to gene expression revealed that $\mathrm{spaC}, \mathrm{spxB}$ and $\mathrm{spaE}$ gene expression was detected equally in active and arrested lesions ( $p>0.05)$, while and wzB gene $(\mathrm{p}=0.006)$ was more prevalent in active dentin lesions. This study provides insights about oral microbiota related to dentin caries activity status, indicating that $L$. casei group has a different behavior in active and inactive lesions and may be linked to dentin lesion progression and activity.

Funded by Cearense Foundation for Scientific and Technological Development Support-FUNCAP.

\section{5}

Association of Adhesin P1 with Salivary AgglutininMediated Adherence in Streptococcus mutans Strains

\author{
J.M. Yang ${ }^{\mathrm{a}, \mathrm{b}}$, A.J. Ligtenberg ${ }^{\mathrm{c}}$, Y.F. Wu ${ }^{\mathrm{d}}$, W. Crielaard ${ }^{\mathrm{b}}$, \\ D.M. Deng*b \\ d.deng@acta.nl \\ aState Key Laboratory of Oral Diseases, National Clinical \\ Research Center for Oral Diseases, West China Hospital \\ of Stomatology, Sichuan University, Chengdu, China; \\ bepartment of Preventive Dentistry, Academic Centre for \\ Dentistry Amsterdam, University of Amsterdam and Vrije \\ Universiteit Amsterdam, Amsterdam, The Netherlands; \\ 'Department of Oral Biochemistry, Academic Centre for \\ Dentistry Amsterdam, University of Amsterdam and Vrije \\ Universiteit Amsterdam, Amsterdam, The Netherlands; \\ dDepartment of Periodontology, West China College of \\ Stomatology, Sichuan University, Chengdu, China
}

The bacterium Streptococcus mutans adheres to salivary agglutinin (SAG) on the dental surface through adhesin P1. Hence, this adhesin is a popular candidate for vaccine development. The variation of P1 in different streptococci has been well documented. However, the variation of this protein in various $S$. mutans strains and the effect on adherence is still unclear. This study aims to link the $\mathrm{P} 1$ protein sequence diversity of 9 S. mutans strains with SAGmediated adherence of these strains. Genomic DNA of $9 S$. mutans strains was isolated for PCR and the P1 gene sequenced. The similarities of the 9 protein sequences were analyzed using the Clustal Omega multiple sequence alignment tool. For adherence analyses, each strain was grown to mid-log phase and the cells were incubated with a SAG-coated surface for $1 \mathrm{~h}$. The amount of adhered bacterial cells was quantified by a metabolic activity assay. The adherence capability of each strain is presented as the percentage relative to the adherence of strain UA159. Out of 9 strains, the P1 sequences of two strains (GS5 and HG723) were identical and contained a stop codon due to one nucleotide insertion. The sequences of the other strains showed at least $97 \%$ similarity. Adherence of strains GS5 and HG723 was $1-3 \%$ of that of the reference strain UA159. The adherence of the other strains varied between $41 \%$ and $185 \%$ of UA159. Strains with highly similar P1 sequences did not demonstrate similar adherence capability. In conclusion, although the P1 protein sequence is conserved among S. mutans strains. variation in adherence capacity exists. Disruption of the P1 protein destroys the adherence capability of S. mutans.

This research was funded by the Department of Preventive dentistry of Academic Center for Dentistry Amsterdam (ACTA). 


\section{6}

\section{Photodynamic Therapy Using Gallium and Curcumin on Streptococcus mutans NCTC10449}

\author{
S.P. Valappil*a, W. Paul' , C.Brown ${ }^{\text {a }}$, C.K. Hope a , S.M. Higham ${ }^{\text {, }}$, \\ C.P. Sharma ${ }^{\mathrm{b}}$
}

S.Valappil@liverpool.ac.uk

aDepartment of Health Services Research and School of Dentistry, University of Liverpool, Liverpool, United Kingdom;

${ }^{b}$ Central Analytical Facility, Biomedical Technology Wing, Sree Chitra Tirunal Institute for Medical Sciences \& Technology,

Trivandrum, India

Antibacterial assay as per the CLSI performance standard was conducted on Mueller-Hinton agar with 5\% sheep's blood which were inoculated with standardised cultures of $S$. mutans NCTC10449, and incubated for $48 \mathrm{~h}$ aerobically at $37^{\circ} \mathrm{C}$. The diameters of zones of inhibition around test samples and controls (Erythromycin; $15 \mu \mathrm{g}$ ) were measured in triplicate. Minimum inhibitory concentration (MIC) of the materials were analysed using broth dilution assay. PDT assay, based on colony-forming-unit (CFU), was conducted on planktonic suspension of S. mutans treated with curcumin, gallium or Cur-c-Gal at concentrations from $0-40 \mu \mathrm{g} / \mathrm{ml}$. Light irradiation with a center wavelength of $450 \mathrm{~nm}$ was applied using an LED (power density of $100 \mathrm{~mW}$ for $5 \mathrm{~min}$ at an energy density of $\left.12.3 \mathrm{~W} \cdot \mathrm{cm}^{-2}\right)$. $\log _{10}(\mathrm{CFU} / \mathrm{ml})$ values were analyzed using one-way ANOVA followed by the Tukey test $(\mathrm{p}<0.05)$ using the SPSS software (New York, USA). Antibacterial assay established zones of inhibition of $S$. mutans to be $18.3 \pm$ $0.3 \mathrm{~mm}$ for Cur-c-Gal, compared with erythromycin $(45 \pm 1.0$ $\mathrm{mm})$, gallium $(29.3 \pm 1.3 \mathrm{~mm})$ and curcumin $(11.0 \pm 0.0 \mathrm{~mm})$. MIC values were established and PDT analyses suggested that the antibacterial effect to be significant $(\mathrm{p}<0.05) ; 86.72 \%$ reduction in CFU compared with a $64.33 \%$ reduction in the absence of PDT. Gallium demonstrated a significant reduction in colony formation, with $12 \mu \mathrm{g} / \mathrm{ml}$ concentration; showing $100 \%$ reduction in CFU both in the presence and absence of PDT $(\mathrm{p}<0.05)$. Curcumin, gallium and Cur-c-Gal displayed growth inhibition of $S$. mutans compared with controls and the antibacterial activity was enhanced in the presence of photosensitizers. This suggest the potential use of this technology in caries treatment.

This work was supported by UKIERI (IND/2012-13/EDU-UKIERI/118).

\section{7 \\ Ratiometric Analysis of pH in Different Layers of 48-h and 120-h Dental Biofilms}

\author{
S. Schlafer*, V. Bælum, I. Dige \\ sebastians@dent.au.dk \\ Department of Dentistry and Oral Health, Aarhus University, \\ Aarhus, Denmark
}

Confocal microscopy based $\mathrm{pH}$ ratiometry allows monitoring $\mathrm{pH}$ development inside dental biofilms in real-time. To date, however, the methodology has only been applied to measure $\mathrm{pH}$ at the bottom of 48-h-old in-situ-grown biofilms. The aim of the present work was to compare $\mathrm{pH}$ development upon exposure to glucose in four different layers of 48-h and 120-h-biofilms. 48-h $(\mathrm{n}=4)$ and 120 -h-biofilms $(\mathrm{n}=3)$ were grown in situ on custom-made glass slabs placed on individually designed lower jaw splints. After removal, the biofilms were incubated with $0.4 \%(\mathrm{w} / \mathrm{v})$ glucose, and extracellular $\mathrm{pH}$ was determined using confocal microscopy imaging of the ratiometric probe C-SNARF-4 and subsequent image analysis. $\mathrm{pH}$ was measured in 9 microscopic fields of view in four different layers of each biofilm, 15 and $45 \mathrm{~min}$ after exposure to glucose. Within the first $15 \mathrm{~min}, \mathrm{pH}$ dropped quickly in all layers of both 48-h and 120-h-biofilms (48-h: $\varnothing=5.91, \mathrm{p}<0.05$; 120-h: $\varnothing=5.67, \mathrm{p}<0.05$; t-test). In the following $30 \mathrm{~min}, \mathrm{pH}$ continued to drop slowly (48-h: $\varnothing=5.74, \mathrm{p}<0.05 ; 120$-h: $\varnothing=5.53, \mathrm{p}=0.07$ ), but the drop was only significant in 48 -h-biofilms. While the average $\mathrm{pH}$ was lower in 120 -h-biofilms after 15 and $45 \mathrm{~min}$, the differences did not reach statistical significance $(\mathrm{p}=0.15 ; \mathrm{p}=0.29)$. Likewise, the average thickness of 120-h-biofilms $(\varnothing=27.16 \mu \mathrm{m}$ $\pm 14.23 \mathrm{SD})$ was higher than the one of 48 -h-biofilms $(\varnothing=15.47$ $\mu \mathrm{m} \pm 4.09 \mathrm{SD})$, but not significantly $(\mathrm{p}=0.17)$. There was no correlation between $\mathrm{pH}$ and biofilm height (person-coefficients ranging from -0.8 to 0.6 ), and under the chosen static conditions no systematic differences between top and bottom layers of the biofilms were observed. In conclusion, the acidogenic potential of 120 $\mathrm{h}$ smooth surface biofilms is only slightly elevated compared to 48-h-biofilms under static conditions.

\section{8 \\ The Use of pH Ratiometry to Measure Extracellular pH in Biofilms of Candida spp.}

J.E. Garcia*a, A. Kamp b ${ }^{\text {, S. Schlafer }}{ }^{\text {a }}$

sebastians@dent.au.dk

aDepartment of Dentistry and Oral Health, Aarhus University,

Aarhus, Denmark; ${ }^{b}$ AIAS, Aarhus Institute of Advanced Studies, Aarhus University, Aarhus, Denmark

Candida spp., such as Candida albicans and Candida dubliniensis have been shown to be implicated in the caries process as part of cross-kingdom biofilms. We have developed a confocal microscopy based methodology that permits to monitor extracellular $\mathrm{pH}$ in microbial biofilms at the microscale in real-time using the ratiometric dye C-SNARF-4. To date, the method has only been employed in bacterial biofilms, and the aim of the present work was to adapt the procedure to allow for quantification of $\mathrm{pH}$ in the matrix of Candida biofilms. To test the staining properties of $\mathrm{C}$ SNARF-4 on fungal cells, we imaged immobilized cells of C. albicans (NCPF 3179) and C. dubliniensis (DSM 13268) with confocal microscopy. Counterstaining with BacLight showed that CSNARF-4 visualized dead cells, as well as the walls of viable cells, leaving the cytoplasm unstained. In 24-h-monospecies biofilms, the same staining pattern was observed. The biofilm matrix showed an intermediate level of fluorescence, and we developed an image analysis procedure with a two-intensity-threshold segmentation to remove all cells from the confocal images and determine extracellular $\mathrm{pH}$. As a proof of principle, we measured $\mathrm{pH}$ developments in monospecies biofilms for $1 \mathrm{~h}$ after exposure to glucose $(0.4 \%$ 
$\mathrm{w} / \mathrm{v})$. Experiments were performed in technical triplicates and biological duplicates. Within 5 minutes, $\mathrm{pH}$ in $C$. albicans biofilms dropped to around $5(\varnothing=5.08)$, whereas $\mathrm{pH}$ drops in C. dubliniensis biofilms were slower and less pronounced, as determined by 2 -sample t-tests $(\varnothing=6.10$ after 5 min; $\varnothing=5.79$ after 60 min; $\mathrm{p}<$ $0.05)$. Only moderate differences between different microscopic fields within the same biofilm were observed and the young biofilms showed no vertical $\mathrm{pH}$ gradients. $\mathrm{pH}$ ratiometry with $\mathrm{C}$ SNARF-4 is a valuable tool to explore $\mathrm{pH}$ developments inside fungal biofilms and potentially cross-kingdom biofilms.

This work was supported by AIAS, Aarhus Institute of Advanced Studies, Aarhus University and Aarhus University Research Foundation (AUFF).

\section{9 \\ Evaluation of Severe Dental Caries Induced by Streptococcus mutans to Development of Infective Endocarditis in Rats}

R. Nomura, M. Otsugu, S. Matayoshi, K. Nakano

rnomura@dent.osaka-u.ac.jp

Department of Pediatric Dentistry, Division of Oral Infection

and Disease Control, Osaka University Graduate School of

Dentistry, 1-8 Yamada-oka, Suita, Osaka 565-0871, Japan

Streptococcus mutans, a major pathogen of dental caries, is considered to be a causative agent of infective endocarditis (IE). Although the bacterium has been detected in heart valves of IE patients, whether its presence in severe dental caries is a possible risk factor for that disease remains unknown. We evaluated the virulence of $S$. mutans harbored in severe dental caries for IE development using a novel rat model. Ten rats (18 days old) were fed a caries-inducing diet containing 56\% sucrose until the end of the experiment to induce severe dental caries. Each received a bacterial suspension of $S$. mutans strain SA31 via the oral cavity once a day for 5 days to colonize the oral cavity. At 90 days old, heart valves in 6 rats were injured using a sterile catheter and wire under general anesthesia, while the remaining 4 did not undergo that procedure. All rats were euthanized at 120 days of age and severe dental caries was confirmed. Next, hearts were extirpated and transversely sectioned, then one half was cut into small pieces and placed onto Mitis-Salivarius-Bacitracin agar plates. Analysis showed that $S$. mutans (approximately $2000 \mathrm{CFU}$ ) was recovered from rats with injured heart valves, whereas no bacterial organisms were recovered from rats without injury. Furthermore, histopathological observation of the remaining halves of the sectioned hearts revealed the presence of $S$. mutans only in rats that underwent the injury procedure. Our findings suggest that $S$. mutans harbored in severe dental caries is a risk factor for bacterial mass formation, especially in injured heart valves, thus contributing to the pathogenicity of IE.

This work was supported by JSPS KAKENHI (grant no. JP15K11363).

\section{0}

The Antimicrobial Profile of Thymus capitatus as Raw Essence and Employing Nanovesicles Targeting Streptococcus mutans and Candida albicans

R. Pinna*a , C. Juliano ${ }^{b}$, M. Manconic, G. D'hallewin ${ }^{\mathrm{d}}$, G. Petretto ${ }^{\mathrm{d}}$, M. Maiolia, , C. Caddeoc, M. Manca ${ }^{c}$, A. Bortonef, P. Usai ${ }^{\mathrm{a}}$, E. Milia ${ }^{g}$

caesareus83@yahoo.it

aDepartment of Biomedical Sciences, University of Sassari,

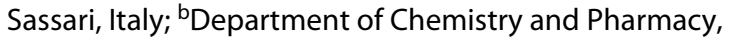
University of Sassari, Sassari, Italy; ' Department of Life and Environmental Sciences, University of Cagliari, Cagliari, Italy;

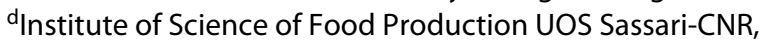

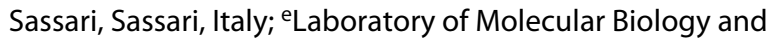
Stem Cell Engineering, National Institute of Biostructures and Biosystems, Bologna, Italy; ${ }^{\mathrm{f}}$ Azienda Ospedaliero-Universitaria, Sassari, Italy; ${ }^{9}$ Department of Clinical and Experimental Medicine, University of Sassari, Sassari, Italy

Antibiotic resistance is an increasing serious issue to global health. As an alternative to chemicals, the use of natural molecules have been proposed to reduce the cariogenicity of Streptococcus mutans, which virulence is increased by the presence of Candida albicans in the oral biofilm. The aim was to assess the antimicrobial capacity of Thymus capitatus (TC) essential oil in the neutralization of $S$. mutans and $C$. albicans and that of three different vesicle nano systems (VNCs) as delivery systems. TC was obtained from the aerial parts of the plant by steam-distillation. Chemical characterization was carried out using gas chromatography. The antimicrobial profile of TC (group A) and that of TC incorporated into liposomes (group B), glycerosomes (group C) and propylen glycol-penetration enhancer containing vesicles (PG-PEVs) (group D) was carried out by disc diffusion assays, MICs, MBC/ MFC and TEM. Planktonic cells culture of S. mutans (ATCC 35668), and C. albicans (ATTC 10231) were used. The cytotoxicity of all the agents was assessed using human primary gingival fibroblasts undergoing $48 \mathrm{~h}$ of exposure using the MTT colorimetric assay. Carvacrol (90.1\%) was the main component of the essential oil followed by linalool (1.1\%), caryophyllene oxide $(1.3 \%)$, p-cymene and terpinen-4-ol (1.1\%), and thymol (1.1\%). The VNCs all demonstrated homogeneous distribution of the particles size and highly negative zeta potential. None of the agents expressed cytotoxicity. Antimicrobial susceptibility discs showed that TC had similar antimicrobial activity to that of VNCs against $\mathrm{SM}(» 12 \mathrm{~mm})$, whereas inhibition was higher toward CA $(12 \mathrm{~mm})$ in comparison to VNCs $(» 10 \mathrm{~mm})$. MBC/MIC ratios demonstrated TC and PG-PEVs exerted antibacterial effects on SA, while only TC effectively killed the yeast as confirmed morphologically. In conclusion the TC oil delivered using PG-PEVs can be useful against S.mutans. In addition, the antifungal activity of TC oil could be valuable as a natural and safe compound to neutralize $C$. albicans.

This study was carried out with the support of the "Fondazione di Sardegna”. 


\section{Sessions 5 and 6 Clinical Studies}

\begin{abstract}
41
Oral and Ocular Dryness Evaluation of Non-Sjögren Patients in the Lublin Region (Poland) Cohort

R. Chałas a , D. Wróbel-Dudzińskab , D. Rykwab ${ }^{\mathrm{b}}$, A. Kubik-Komar', T. Żarnowskib

drrzenia@wp.pl

aDepartment of Conservative Dentistry and Endodontics, Medical University of Lublin, Lublin, Poland; ${ }^{b}$ Department of Diagnostics and Microsurgery of Glaucoma, Medical University of Lublin, Lublin, Poland; 'Department of Applied Mathematics and Computer Science, University of Life Sciences in Lublin, Lublin, Poland
\end{abstract}

The aim of this study was to first demonstrate an application of the modified Schirmer tests for salivary gland hypofunction in non-Sjögren patients. The comparison and correlation the results achieved from the eye Schirmer test and modified Schirmer tests with the healthy control group was the second objective. The third aim was to determine the correlation between those tests and subjective and objective symptoms of the ocular surface and mouth dryness. The study was conducted on 642 patients (405 females and 237 males) from the Lublin district area (Poland) with or without subjective or/and objective symptoms of dry eye or mouth who did not fulfil the criteria for diagnosis of Sjögren syndrome. Patients were divided on the basis of history and comprehensive oral and ocular examination into the different groups. The eye Schirmer test (ST) and the modified mouth Schirmer tests (MST) were performed. The results were recorded after 1 minute and 3 minutes for MSTs and 5 minutes for ST. The eye ST and modified Schirmer tests scores were considerably higher in the healthy group than in other, ( $p<0.001$ for each group combinations). The results of MST tests decreased with the appearance of subjective and objective symptoms $(\mathrm{p}<0.001)$. The differences between the results obtained by ST and MSTs between the groups were highly significant $(\mathrm{p}<0.001)$. There were positive correlations between ST and MST outcomes between the groups $(\mathrm{p}<0.001)$. This is the initial report comparing the Schirmer test results in eye and mouth simultaneously performed in non-Sjögren symptomatic patients vs asymp- tomatic healthy individuals. According to the achieved results, the modified Schirmer tests seem to be rapid, convenient and reliable objective screening tools for salivary gland hypofunction in nonSjögren patients.

\footnotetext{
42

Gender Differences in Self-Evaluation and Implementation of Flossing Skills

S.-C. Schmidt ${ }^{\text {a }}$ K. Giese-Kraft*a, N. Schlueter ${ }^{\text {b }}$, C. Ganss ${ }^{\text {a }}$

katja.giese-kraft@dentist.med.uni-giessen.de aDepartment of Conservative and Preventive Dentistry, Dental Clinic, Justus-Liebig-University, Giessen, Germany ${ }^{b}$ Division for Cariology, Department of Operative Dentistry and Periodontology, Center for Dental Medicine, Medical Center - University of Freiburg, Faculty of Medicine, University of Freiburg, Freiburg im Breisgau, Germany
}

So far, gender aspects have been rarely addressed in oral hygiene education. The aim was therefore to investigate gender-related effects of a video-instruction on self-evaluation of flossing skills and performance of flossing. A convenience sample of students ( $\mathrm{n}=97,31$ male, 66 female, $24.3 \pm 3.0$ years) not involved in medicine/dentistry was included and received an instruction how to floss correctly. At baseline, after instruction (T2) and a third time (7-day intervals, T3) subjects flossed while being video-filmed through a mirror and answered a questionnaire. Videos were analysed with observation software INTERACT regarding reached proximal spaces and correct flossing technique (vertical strokes, proper handling, and cleaning mesial and distal surfaces). Questionnaires were based on an analogue scale (0-10) assessing selfrating of subjects as to reached proximal spaces. Statistics: MannWhitney-test, Spearman-correlation coefficients, cross-tabulations and chi-square-test. At baseline, less than 20\% showed a correct flossing technique with no significant differences between females and males (vertical strokes: 19.7\%/16.1\%; proper handling: 6.1\%/16.1\%; mesial-distal: $18.2 \% / 19.4 \%$ resp.). The number of reached spaces was similar for both genders. At T2, more fe- 
males showed a correct flossing technique compared to males (vertical strokes: $73.8 \% / 43.3 \%$; $\leq 0.01$; proper handling: $96.9 \% / 80.0 \%$; $\mathrm{p} \leq 0.05$; mesial-distal: $92.3 / 80.0$; n.s.) but there was no difference as to reached spaces. At T3, females did not improve further as to technique, but males reached levels of females. At all time points, self-evaluation and video-observed number of reached proximal spaces correlated better in males than in females (baseline: 0.71/0.56; T2: 0.51/0.37; T3: 0.55/0.32 resp.). Females implemented technique more efficiently than males, but performed similar as to reached proximal spaces. However, females assessed themselves inferior to the factual results while males evaluated themselves more realistically. Gender aspects might be relevant in oral hygiene instructions.

Funded by Justus-Liebig-University, Giessen.

\section{3 \\ Level of Enamel Temperature During $\mathrm{CO}_{2}$ Laser Irradiation $\left(100-500^{\circ} \mathrm{C}\right)$ Influences Erosion Resistance}

M. Esteves-Oliveira*a, J. Glökler ${ }^{\mathrm{a}}$, S. Wollgarten ${ }^{\mathrm{b}}$, J. Stollenwerk ${ }^{\mathrm{c}}$, R. Poprawe ${ }^{\mathrm{b}, \mathrm{c}}$ H. Meyer-Lueckeld

mestevesoliveira@ukaachen.de aDepartment of Operative Dentistry, Periodontology and Preventive Dentistry, RWTH Aachen University, Aachen, Germany; ${ }^{b}$ Chair of Laser Technology, RWTH Aachen

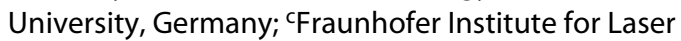
Technology (ILT), RWTH Aachen University, Germany; dDepartment of Restorative, Preventive and Pediatric Dentistry, zmk bern, University of Bern, Switzerland

The increase of enamel acid resistance after $\mathrm{CO}_{2}$ laser irradiation seems to be related to the increase of enamel temperature. Therefore, the aim of the present study was to investigate the effect of several surface temperature levels $\left(100-500^{\circ} \mathrm{C}\right)$ caused by laser irradiation on enamel erosion resistance. Ninety-six polished bovine enamel samples were randomly allocated to one of six groups $(\mathrm{n}=16)$ : no treatment, control $(\mathrm{C}) ; \mathrm{CO}_{2}$ laser irradiation heating up enamel to $100{ }^{\circ} \mathrm{C}$ (L100), $200^{\circ} \mathrm{C}$ (L200), $300{ }^{\circ} \mathrm{C}$ (L300), $400{ }^{\circ} \mathrm{C}$ (L400), and $500{ }^{\circ} \mathrm{C}$ (L500). CO2 laser irradiation was performed at $10.6 \mu \mathrm{m}$ wavelength $\left(20 \mu \mathrm{s} ; 0.1-1.3 \mathrm{~J} / \mathrm{cm}^{2} ; 2-8 \mathrm{KHz}\right)$ and over an area of $7 \times 2 \mathrm{~mm}$ (automatic scanning modus). After surface treatment samples were submitted to an erosive cycling over six days, including immersion in citric acid $(2 \mathrm{~min} / 0.05 \mathrm{M} / \mathrm{pH}=2.3)$ six times daily and storage in remineralization solution $(\geq 1 \mathrm{~h})$ between erosive attacks. After treatment (baseline) and at the end of four cycling days (D) namely, D2, D4, D6 ad D10 enamel surface loss $(\mu \mathrm{m})$ was measured using a 3D laser profilometer. Data were statistically analyzed with a two-way repeated measures ANOVA and post-hoc comparisons $(\alpha=0.05)$. At D10 temperature levels from $100-500{ }^{\circ} \mathrm{C}$ caused signifcant increase in enamel erosion resistance as compared to $C(-31.6 \pm 2.9 \mu \mathrm{m}, \mathrm{p}<0.05)$ and a linear decrease in substance loss was observed with increasing temperatures $\left(\mathrm{r}^{2}=0.9717\right)$. L500 enabled the lowest substance loss $(-10.9 \pm$ $2.8 \mu \mathrm{m})$, which was also significantly lower than for all other laser groups $(\mathrm{p}<0.05)$. Between L400 $(17.1 \pm 3.3 \mu \mathrm{m})$ and L300 $(-19.3 \pm$ $2.7 \mu \mathrm{m})$ no significant difference was observed ( $\mathrm{p}>0.05)$. In con- clusion laser heating of enamel from 100 to $500{ }^{\circ} \mathrm{C}$ levels indeed significantly increased enamel erosion resistance in vitro and the highest erosion reduction compared to the non-treated control was observed for L500.

This study was funded by the DFG (German Research Foundation, Grants Nr.: ES 426/2-1, PO 591/40-1).

\section{4 \\ New Caries Risk Assessment System "CRASP" - Report from a Japanese Private Dental Clinic}

\section{S. Sugiyama}

sdcss@pastel.ocn.ne.jp

Sugiyama Dental Clinic, Tokyo, Japan

Caries Risk Assessment (CRA) is one of the important components in the caries management, but it is not common in Japan. Sugiyama Dental Clinic (SDC) first adopted CRA based on Dentocult system in 1995, but the continuous use in practice was difficult because of the cost. This time we developed a new CRA system, Caries Risk Assessment Share with Patient (CRASP). CRASP is a simple system, focusing on sharing clinical information with patients, at low cost. CRASP assesses the fluoride usage, dietary habit by questionnaire on a computer screen, plaque control, filling due to caries within one year, and caries activity test (Cariostat, Willdent Japan). These data can be input into the database at chair side. The patients and their family can visually see the result of the assessment, caries risks indicated by color - blue (low), yellow (medium), red (high) upon data input. We started applying CRASP to both maintenance and treatment patients (excluding one-time emergency patients) in 2017 , and the implementation rate was $67.4 \%$ after 12 months (1106 out of 1640 patients, 4 - to 88 -yearold). In comparison between under 19-year-old (U19) and 60- to 79 -year-old patients (G60), there were some risk factors in G60; over three times rinsing after brushing (U19: 18.4\%, G60: 54.7\%), not enough fluoride toothpaste (U19: 18.0\%, G60: 47.8\%), and high caries activity indicated by Cariostat (U19: 7.4\%, G60: 18.4\%); meanwhile, other risk factors were more prominent in U19; sweet beverage at home (U19: 18.4\%, G60: 12.2\%), and not enough plaque control (U19: 79.3\%, G60:52.9\%). In conclusion, we implemented CRASP into more than half patients and shared the caries risk with patients. CRASP may become a common CRA procedure in Japanese dentistry after suitable validation.

This study was supported by The Japan Health Care Dental Association. 
45

\section{Selective Carious Tissue Removal Using Hand Excavation or Polymer Burs: Preliminary Results of a RCT}

M.G. Marques a, K.M. Demarchi ${ }^{\mathrm{a}}$, S.C. Leal', A.P.D. Ribeiro ${ }^{\mathrm{a}, \mathrm{b}}$, F. Schwendicke ${ }^{c}$, L.A. Hilgert*a

leandrohilgert@gmail.com

aUniversity of Brasília, Brasília, Brazil; bUniversity of Florida, Gainesville, USA; ${ }^{C}$ Charité Universitä tsmedizin Berlin, Berlin, Germany

The aim was to compare immediate outcomes of selective caries removal of deep lesions in primary molars using subjective (hand instruments) and objective (polymer burs) protocols. 115 6-7 year-old children presenting radiographically inner-half dentin carious lesions in teeth with vital pulps were randomized into groups $\mathrm{S}$ (subjective) or $\mathrm{O}$ (objective). 177 teeth $(\mathrm{S}, \mathrm{n}=91$ and $\mathrm{O}$, $\mathrm{n}=86$ ) were treated by two trained dentists. Carious dentin selective removal was performed using hand instruments (S) or a selflimiting polymer bur (O, Polybur, Komet). All cavities were restored with a high-viscosity glass-ionomer (Equia Forte, GC). Immediate post-treatment data: pulpal exposures, need of local anesthesia, treatment time, patients satisfaction (5-points Likert faces scale) were recorded. Generalized linear multivariable mixed models, accounting for multi-layer clustering, were used for statistical analysis. No pulpal exposures occurred. Only two restorations required anesthesia. Treatment times [Mean $(95 \% \mathrm{CI})$, S:432.95 s(404.02-461.87) and O:411.63 s(382.50-440.76)] were not significantly different between protocols $(\mathrm{p}=0.378)$. Posttreatment patients satisfaction was high (median score 1: very satisfied) for both selective carious tissue removal protocols, with no significant difference between them $(\mathrm{p}=0.164)$. Concerning occurrence of pulpal exposures, need of local anesthesia, treatment time, and patients immediate post-treatment satisfaction, there are no significant differences between hand excavation and polymer burs for selective carious tissue removal. Restorations longevity and possible complications will be analyzed at the RCT followups.

This study was funded by FAPDF, FINATEC, University of Brasília and Komet. Polymer burs were donated by Komet. HVGIC was donated by GC.

\section{6}

\section{Benefits of Using Virtual Reality in Cariology Teaching}

H.H. Hamama*, K.Y. Tse, S. Murbay

hamdy@connect.hku.hk

Faculty of Dentistry, The University of Hong Kong, Hong Kong S.A.R., China

This study was designed to test the hypothesis of that students who receive virtual reality training prior to practicing on natural teeth exhibit high self-confidence levels compared to their peers who receive post-practice VR training. The Null-hypothesis is that
Virtual reality training cannot totally replace the 'traditional' cariology teaching methods. A total number of 76 students voluntarily participated on this study during their normal cariology course practical sessions. The students were assigned into 2 groups ( 38 each). In the test group, students performed the caries excavation on haptic machines (Cariology software, Simodont Dental Trainer, Moog Dental Simulation, NY, USA) prior to practicing on extracted natural carious teeth. Conversely, in the control group, students were exposed to virtual reality training after practicing on extracted natural teeth. An evaluation questionnaire, prepared by professional applied psychologist, was disseminated among the students to measure their satisfactory and confidence levels. The students were informed to anonymously complete this questionnaire and they were allowed to express their feelings about overall learning experience through an open-end question at the end of the questionnaire. Students were informed that their feedback will be used for course development/research purposes. The results of chi-square test showed no significant difference in responses among the two groups ( $p>0.05)$. Nevertheless, the responses of both groups showed that virtual reality is considered as a useful educational tool, most of the students (92.5\%) superiorly ranked experience gained from practicing on natural carious teeth compared to VR. Furthermore, they clearly stated that virtual reality allowed them to practice more and improve their self-confidence level as well as eye-hand coordination. In light of the results of this study, the null-hypothesis of this study was accepted. In conclusion Virtual reality is a very useful adjunct tool to conventional caries excavation practice on extracted natural teeth. Although this method has numerous advantages, it cannot provide the same tactile sensation compared to conventional caries excavation on natural teeth.

\section{7 \\ Differences on Children's Treatment Planning When Detecting Initial and Moderate Active Caries: A Before-and-After Study
I. Floriano*a,b, R.A. Gomes ${ }^{\mathrm{a}}$, G.M. Machado ${ }^{\mathrm{a}}$, N.M. Ladewig ${ }^{\mathrm{a}}$, E.S. Rocha ${ }^{\mathrm{a}}$, R.A. Pereira ${ }^{\mathrm{a}}$, T.K. Tedesco ${ }^{\mathrm{a}, \mathrm{c}}$, L. Yoshioka ${ }^{\mathrm{a}}$, D.P. Raggio a , F.M. Mendes ${ }^{\mathrm{a}}$, M.M. Braga ${ }^{\mathrm{a}}$, CARDEC Collaborative Group a \\ isabelafloriano@usp.br \\ aDepartment of Pediatric Dentistry, University of São Paulo, São Paulo, Brazil; bentistry Course, Uninovafapi University Centre, Piauí, Brazil; ' Dentistry Course, Ibirapuera University, São Paulo, Brazil}

This before-and-after study evaluated changes observed in treatment planning when active initial and moderate caries lesions in primary teeth were considered to be treated in decision-making. This study is nested in a RCT (NCT02473107, CARDEC-02). 3-to6-year-old children allocated to one arm of this trial were examined twice using ICDAS: $1^{\text {st }}$ ) only moderate and extensive caries lesions were recorded despite their activity status; $2^{\text {nd }}$ ) the exam included initial lesions and activity status assessment. The same operator prepared 2 treatment plans based on examinations using guidelines established for the trial. Then, both plans were com- 
pared looking for cases in which, using the $2^{\text {nd }}$ strategy, non-operative treatment were added and/or operative treatments avoided. Poisson regression analyses were used to test the impact of diagnosis strategy on child treatment. The prevalence ratios were calculated for each case with 95\% CI. Expected costs for the planned treatment were compared with paired t test. 130 children were included. Only $6 \%$ presented no caries lesions. Detection and treatment of initial active lesions would increase the need for non-operative treatment by $35 \%$. Changes were less common in children with previous caries experience $(\mathrm{dmft}>0)$. The activity assessment of ICDAS score 3 lesions would save operative treatments for only 6 patients (5\%). Treating active initial and moderate lesions results in a slightly additional expense with non-operative procedures (mean: US\$1.67 \pm 3.20 ) while a higher amount could be saved by avoiding some operative interventions (mean: US\$11.75 \pm 30.21 ). Savings could be even higher if only children with $\mathrm{dmft}>0$ were considered (mean: US $\$ 16.18 \pm 4.70$ ). In conclusion, detecting and treating active initial and moderate caries lesions can reasonably modify the management of dental caries lesions in primary dentition and a possible reduction in costs could be expected.

Funded by Fapesp (2013/27206-8) and CNPq (448013/2014-2) supported this study.

\section{8 \\ Antibacterial Effect of a Bioactive Cement with Selective Caries Removal - An In-Vivo Study}

J. Schmidt*a, S. Krohn b , L. Buenger ${ }^{\mathrm{a}}$, T. Berg' ${ }^{\text {b }}$, F. Krause ${ }^{\mathrm{a}}$, K.J. Park ${ }^{\mathrm{a}}$, H. Schneider ${ }^{\text {a }}$ D. Ziebolz ${ }^{\text {a }}$ R. Haak ${ }^{\text {a }}$

jana.schmidt@medizin.uni-leipzig.de

aDepartment of Cariology, Endodontology and

Periodontology, University of Leipzig, Leipzig, Germany;

bepartment of Gastroenterology and Rheumatology, Section

Hepatology, University of Leipzig, Leipzig, Germany

This study investigated the antibacterial effect of a bioactive cement on carious dentin after selective caries removal. Microbial community profiling of carious lesions was performed. In this randomized, controlled clinical trial 11 patients were included showing deep primary carious lesions at two teeth without pulpal symptoms that radiographically extend up to the inner third of dentin. At baseline visit (BL) carious tissue was selectively removed and carious dentin was sampled with a standardized sterile roundbur. A bioactive cement (Biodentine, BD; Septodont, Paris) or guttapercha as control (CTR) were randomly applied before sealing (Optibond FL, Kerr, Rastatt) and restoration with a composite filling (SDR, Ceram X; DENTSPLY DeTrey GmbH, Konstanz). At re-entry (RE) after 8 weeks carious dentin was sampled again. By DNAse degradation prior bacterial DNA isolation, only DNA from intact cells was detected and quantified with $16 \mathrm{~S}$ rRNA gene based PCR per roundbur load. Terminal-restriction fragment length polymorphism analysis (tRFLP) was used for microbial community profiling. In both treatment groups, fewer intact bacterial cells $\left(\mathrm{BD}: \mathrm{BL}=7.8^{*} 10^{6}, \mathrm{RE}=7.5^{*} 10^{3}\right.$; CTR: $\mathrm{BL}=2.9^{*} 10^{6}$, $\mathrm{RE}=3.1^{*} 10^{5}$ ) were found after 8 weeks (BD: $\mathrm{p}=0.000275$; CTR: $p=0.033$ ). However, the decrease of the bacterial DNA content was significantly higher if the carious dentin was covered with BD compared to CTR $(\mathrm{p}=0.028)$. Considering the microbial composition at BL intra-individual differences between the lesions were found in 8 of 9 considered patients. Regardless of the treatment strategy, a microbiological shift from BL to RE was detected. In conclusion Biodentine had an additional antibacterial effect after selective caries removal. The microbiological composition in carious lesions differs intra-individually and over time. TRFLP is suitable for investigating differences in the microbial community of carious lesions.

The authors thank Septodont for financial support. This study was supported by the dgpzm CP GABA Science Fund.

\section{9 \\ A Comparative Study of Dental Caries Prevalence in Former Orthodontic Patients}

\author{
E. Boteva $a^{\text {a* }}$ G. lordanova \\ e_boteva@abv.bg \\ ${ }^{a} \mathrm{NCL}$ Conservative Dentistry, ${ }^{\mathrm{b}} \mathrm{D}$. Orthodontics, Faculty of \\ Dental Medicine, Sofia, Bulgaria
}

In the 21 century dental caries is not a multifactorial disease on an individual level and community approaches are far away in the past. A dental caries process can be "hidden" for a few years on a subclinical level. The caries experience outcomes after orthodontic treatment has led to several publications in 30 years with contradictory results. The aim of the present study is to evaluate dental caries experience in subjects aged 21.39 years, dental students, in relation to previous orthodontic treatments. 98 Subjects ( 60 female and 38 male), 38 of them with various orthodontic treatments before the age of 18 attend the survey. Questionnaire: form with 14 questions was filled. Fixed and removable appliances were not recorded separately due to the relative compensation between the intraoral time and volume of retentive surfaces. D, M, F, T counts were recorded separately, gingivitis or gingival bleeding were also recorded. Exclusion criteria: chronical diseases and long term medication. Radiographs for diagnostic purposes. The significantly lower DMFT scores $(t=6,0085, \mathrm{p}<0.001)$ among orthodontically treated volunteers shows high quality of dental hygiene education and practices. Among volunteers with past orthodontic treatments the proportion of those who don't snack sweets and candies between meals is higher. The former female orthodontic patients are with lower DMFT scores related to higher fluoride retention, in all volunteers caries activity is in low proportions (7.7$11.7 \%)$. Specification of brushing, rinsing and washing methods have to be individually specified related to DMFT counts, to dental caries activity. The follow up caries occurrence in orthodontic patients few years after the treatment is essential. 


\section{0}

\section{One Year Clinical Follow-Up of Stepwise Technique in Deep Dentin Carious Lesions}

\section{S. Oglakcioglu*, T. Pamir}

oglakcioglusinem@gmail.com

Department of Restorative Dentistry, Ege University, Izmir, Turkey

The purpose of this clinical follow-up was to evaluate the stepwise excavation technique in deep dentin lesions. Fifteen permanent teeth with caries lesions, penetrating $75 \%$ or more into dentin, were included. Periphery of cavities including enamel-dentin junction was cleaned with low speed steel bur, until hard dentin was reached. Selective removal to soft dentin over the pulp was manually applied in the first step excavation. Following excavation to soft dentin, cavities were covered with pure calcium hydroxide and zinc oxide eugenol cement (Alganol, Kemdent ${ }^{\circledR}$ ). Conventional glass ionomer cement (KetacTM Molar Quik Applicap, 3M ESPE) was used for temporary filling. Clinic and radiographic evaluations were performed at the 6th month according to the clinical signs (i.e. vitality, pain/discomfort, sensitivity to percussion or bite test) and radiographical signs as per periapical index of Dag Orstavik. Then, cavities were re-entered and selective removal was applied to firm dentin. Permanent restorations were performed with resin composite (G-aenial ${ }^{\circledR}$ Posterior, GC). As a result of the re-entry procedure, pulp exposure occurred in the $54 \%$ of teeth. Root canal therapy was applied to two teeth due to chronic pulp inflammation whereas other five teeth survived with direct pulp capping. At the end of 12 months, thirteen teeth were still vital without any clinical and radiographical complications. It was concluded that stepwise excavation led to pulp exposure more than expected and it may be better to reconsider re-entry.

There is no company support. Materials are the routines of the restorative department of university.

\section{1}

\section{Fluoride on the Oral Mucosa and in Saliva - A Pilot Study}

\section{Staun Larsen*, A. Richards, B. Nyvad}

line.staun@dent.au.dk

Department of Dentistry and Oral Health, Aarhus University, Aarhus, Denmark

For control of caries fluoride has to be present in the liquid phase surrounding the teeth. Therefore, knowledge on potential fluoride reservoirs contributing to maintaining an elevated concentration of fluoride in the oral fluids are of interest. The oral mucosa may represent a reservoir for fluoride. One study, in a few individuals, showed higher fluoride levels on buccal mucosa than in mixed saliva after a fluoride mouth rinse. The aim of this study was to evaluate and compare fluoride concentrations on the oral buccal mucosa with fluoride concentrations in unstimulated whole saliva in 12 healthy volunteers who had brushed with 1,450 ppm fluoride toothpaste 1-2 hours earlier. Unstimulated saliva was col- lected over a 5-minute period and mucosa samples were collected by scraping the buccal mucosa (avoiding the parotic duct) with a custom made spatula (acetate film). All samples were analyzed with a fluoride electrode adapted for microanalysis following centrifugation, dilution (mucosa samples only) and addition of TISABIII (1:10). Fluoride concentrations on the buccal mucosa were higher than those measured in saliva for all subjects. The mean fluoride concentration from mucosa samples was $0.39 \mathrm{ppm}(95 \%$ CI 0.31 to 0.47 ), and for saliva samples $0.12 \mathrm{ppm}$ (95\% CI 0.05 to 0.18 ) (paired t-test $\mathrm{p}<0.0001)$. We conclude that the concentration of fluoride on the oral buccal mucosa is approximately three times higher than the fluoride concentration in unstimulated mixed saliva 1-2 hours after brushing with regular fluoride toothpaste. More studies are required to explore the relationship between fluoride in saliva and on the oral mucosa, and to investigate to which extent the relationship may vary with time after tooth brushing and concentration of fluoride in the toothpaste.

\section{2 \\ Lesion Activity in Cavitated Carious Lesions in Primary Teeth - A Clinical Scoring System \\ E. Garcia ${ }^{\mathrm{a}}$, S.C. Leal ${ }^{\mathrm{a}}$, L. Bjørndal*b \\ labj@sund.ku.dk \\ aDepartment of Dentistry, Faculty of Health Science, University of Brasília, Brasília, Brazil; ' Department of Odontology, \\ University of Copenhagen, Copenhagen, Denmark}

A 1-step selective carious removal procedure has gained evidence to manage deep carious lesions in primary teeth. However, the potential influence of lesion activity prior intervention toward outcome is unclear. The aim of this clinical pilot study was to test if a preoperative caries activity scoring system can differentiate carious lesion activity in cavitated primary molars. Clinical assessment followed by the analysis of 5 cavities in primary molars presenting different clinical conditions were used to construct an activity point scoring system: Biofilm inside cavity [complete covering 2 points(p), partly $1 \mathrm{p}$; no biofilm $0 \mathrm{p}$ ], central and peripheral carious dentine colour (yellow/light brown 2p, dark brown/black $1 \mathrm{p}$ ), moisture (wet $2 \mathrm{p}$, dry $0 \mathrm{p}$ ), dentine hardness (soft $2 \mathrm{p}$, firm/ leatherly $1 \mathrm{p}$, hard $0 \mathrm{p}$ ), EDJ demineralization (present $2 \mathrm{p}$, not present $0 \mathrm{p}$ ). Combined radiographic lesion depth (RLD) of dentine thickness (\%) and child age: RLD >50\% (2p), 3-4 yr (4p); RLD $>50 \%$ (2p) 5-6 yr (3p); RLD < 50\% (1p) 3-4 yr (2p), RLD < $50 \%(1 \mathrm{p}), 5-6 \mathrm{yr}(1 \mathrm{p})$. The intervals of 5-11p and 12-18p, defined low and high activity scores, respectively, by dividing the scale in halves. The examiners used the system to assess 12 cavities showing excellent intra- and inter-examiner reliability $(\mathrm{k}>0.90)$. The mean scores of $9.2 p$ defined relatively slow progressing lesions, whereas the high lesion activity had a mean of 13.2p. In conclusion, it seems possible to differentiate lesion activity prior intervention in cavitated lesions. The potential influence on this variable needs further investigation for longevity of restorations as well as pulp complications.

This study was partially supported by the National Council for Scientific and Technological Development of the Brazilian Government, Grant 306852/2016. 


\section{3}

\section{French Dentist's Restorative Treatment Decisions in Immature Permanent Molars}

\author{
M. Muller-Bolla*a, C. Coulot ${ }^{\text {b }}$ S. Doméjean ${ }^{c}$ \\ muller@unice.fr \\ aUFR d'Odontologie de Nice Sophia Antipolis, CHUN Fr, 2 \\ Laboratory URB2i EA 4662, Paris Descartes, Montrouge, France; \\ bUFR d'Odontologie de Nice Sophia Antipolis, France; 'UFR \\ d'Odontologie, CROC EA 4847, Université Clermont Auvergne, \\ CHU Clermont-Ferrand, France
}

If many questionnaire surveys all around the globe investigated the management of carious lesions in adults, there is a lack of data in children in general and on immature permanent (IP) teeth in particular. The aim of the present questionnaire survey undertaken among French general practitioners (GPs) who regularly treat children (pediatric dentistry is not a specialty in France) was to investigate the management decisions for approximal and occlusal carious lesions in IP molars. Design - A newly developed questionnaire was sent by e-mail to the members of the French Society of Pediatric Dentistry $(\mathrm{n}=250)$. The structured questionnaire assessed the restorative thresholds and management strategies of various carious conditions on IP molars. Statistical analyses (descriptive, $\mathrm{X}^{2}$ ) were performed. The response rate was $80.4 \%$. Restorative thresholds differed markedly for occlusal and approximal lesions. Indeed, respectively $13 \%$ and $58 \%$ of the respondents would surgically intervene for occlusal (ICDAS 1-2) and approximal (on radiograph) enamel lesions that could have benefit from non-invasive therapies. The preferred restorative material, whatever the lesion severity and the site (occlusal versus approximal) considered, was composite (79.5\%). Respectively $8 \%$ and $5.5 \%$ of the respondents would use glass ionomer cements for the occlusal and approximal lesions in a child with risk factors under control; still $2 \%$ would prefer amalgam restorations. Management decisions were not influenced by respondent demographic characteristics (age, gender, type of practice). The study, the first of its nature in France, shows the need to develop minimum intervention dentistry based on scientific evidence in particular for approximal carious lesions in IP molars.

\section{4 \\ Children's Discomfort in Deep Caries Lesion Management - A Randomized Clinical Trial}

T.K.Tedesco*a,b , G.S.Q. Silva ${ }^{\mathrm{b}}$, T. Gimenez ${ }^{\mathrm{a}, \mathrm{b}}$, A.C.V. Mello-Moura ${ }^{\mathrm{b}}$, G. Machado ${ }^{b}$, J.P. Conceiçãao ${ }^{b}$, I. Floriano ${ }^{c}$, D.P. Raggio a

tamarakt@usp.br

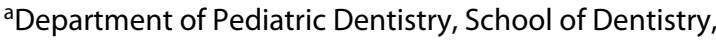

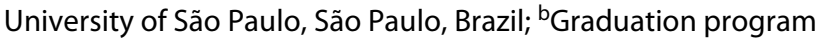
in Dentistry, Ibirapuera University, Sao Paulo, Brazil; 'School of Dentistry, University center of Uninovafapi, Teresina, Brazil

The aim of this randomized clinical trial (NCT02903979) was to evaluate the impact of different restorative techniques to treat deep caries lesion of primary molars in children's discomfort. 4-8 years-old children with at least an occlusal or occlusoproximal deep caries lesion in primary molar were selected from Pediatric Dentistry Clinic of the Ibirapuera University, Sao Paulo, Brazil. Teeth were randomized into two groups, according to the intervention on deep caries lesions: (1) High-viscosity glass ionomer cement (HVGIC) restoration or (2) restoration with hydroxide calcium cement with posterior HVGIC (HCC). Immediately after the final caries management, children reported their discomfort during the restorative technique to an external examiner by WongBaker faces scale. One-way ANOVA were performed to compare the means of children's discomfort between the groups. The level of significance adopted was $5 \%$. One hundred and eight children fulfilled the eligibility criteria and were randomized in the two groups $(n=54)$. Most of children that received HCC restorations reported no or minimal discomfort (score 0) (83.3\%), whilst $92.6 \%$ of the children treated with HVGIC restoration reported similar score. ANOVA showed no statistical significance difference between the restorative techniques ( $p \geq 0.05)$. Mean of children's discomfort for HCC restorations was 0.37 (1.01). For HVGIC restoration group, the mean was 0.41 (1.01). It can be concluded that both restorative techniques result in no or minimal children's discomfort for deep caries lesion management.

Funded by Conselho Nacional de Desenvolvimento Científico e Tecnológico (CNPq - Process 423184/2016-4).

\section{5 \\ Longitudinal Study on Sealing of Progressing Occlusal Caries Lesions in Adolescents}

P.F. Bittencourt*a, N.R. Aimee ${ }^{\mathrm{a}}$, A.C. Queiroz ${ }^{\mathrm{a}}$, H.D. Mestrinho ${ }^{\mathrm{a}}$, J.C. Carvalho

patriciafernanda@hotmail.com

aFaculty of Health Sciences, University of Brasília (UnB), Brasília, Brazil; bCatholic University of Louvain (UCL), Brussels, Belgium

The aim of this study was to assess after 1 year the efficacy of sealing progressing occlusal caries lesions in Brazilian adolescents enrolled in a comprehensive Oral Health Care Program. At the 2-year follow-up of the controlled clinical trial $(n=500), 46$ adolescents, 12-17 year-old, showed caries progression that potentially could be treated with sealants. A total of 53 occlusal lesions clinically diagnosed as active non-cavitated lesion (5.7\%), small active cavitated lesion (66.0\%), inactive non-cavitated lesion (9.4\%) and small inactive cavitated lesions (18.9\%) were all indicated for sealing. These occlusal lesions were radiographically assessed as no radiolucency (17.0\%), radiolucency at the enamel dentine junction $(32.0 \%)$, at the outer third of dentine $(34.0 \%)$ and at the middle third of dentine (17.0\%). Progression was established either clinically, radiographically or both. Reliability for clinical and radiographic assessments were 0.79 (95\% CI 0.73-0.84, nonweighted Kappa) and 0.91 (95\% CI 0.88-0.94, weighted kappa), respectively. Sealant application was preceded by tooth prophylaxis and isolation with cotton rolls. The sealant was applied on specific occlusal sites that showed caries progression. Occlusion was checked and adjusted if required. After 1 year, 4 patients moved to another city ( 4 teeth) and 5 others ( 5 teeth) had their teeth restored by another dentist. For the remaining 44 teeth, the clinical examination showed that $79.6 \%(\mathrm{n}=35)$ sealants were op- 
timal, $9.0 \%(n=4)$ were partially lost and in need of repair, $4.6 \%$ $(\mathrm{n}=2)$ were totally lost and in need of replacement by a new sealant and other $6.8 \%(\mathrm{n}=3)$ in need of restoration. The radiographic assessment of the lesions indicated that the majority of the lesions presented no changes $(88.6 \%), 2$ lesions showed regression $(4.6 \%)$ and 3 other showed progression (6.8\%). In conclusion, sealing seemed to be an effective strategy for arresting progressing non-cavitated and small cavitated occlusal lesions, but a closer monitoring is required.

Funded by The Brazilian National Council for Scientific and Technological Development (CNPq), Brazil, Grant number 445982/2014-4.

\section{6 \\ Secondary Caries Risk in Patients with Fixed Dental Prostheses}

A. Mijoska*a , N. Stavreva a , J. Nikolovska ${ }^{\text {a }}$ E. Zabokova-Bilbilovab, A. Sotirovska lvkovskab

amijoska@yahoo.com

aFaculty of Dental Medicine, University St. Cyril and Methodius, Majka Tereza, PO BOX 17, 1000 Skopje, Republic of Macedonia; bUniversity Dental Clinical Centre Ss Panteleimon in Skopje, Skopje, Macedonia

Secondary caries in the margin region of the abutment teeth in fixed dental prostheses (FDP) is an unwanted complication which may lead to premature tooth extraction. Poor oral hygiene in the buccal region leads to plaque accumulation, and a poorly fitting margin favors the initiation of secondary caries. The aim of the study was to evaluate the secondary caries risk, in teeth undergoing prosthetic restorations by determining the Plaque Formation Rate Index (PFRI) and $\mathrm{pH}$ of the adhering plaque. 30 patients from the University Dental Clinic with fixed four unit prostheses in the molar region on the one side of the dental arch, and healthy natural teeth on the contralateral side were recruited. We determined the PFRI score and plaque $\mathrm{pH}$ on the both sides. Plaque Indicator Kit (GC) was used for determination of the plaque $\mathrm{pH}$ as a caries risk factor. $\mathrm{pH}$ below 5.5 suggested increased bacterial lactic acid producing activity and hard tissue demineralization. The mean value of the plaque $\mathrm{pH}$ in FDP side was $6.16 \pm 0.30$ correlated to natural teeth group $6.75 \pm 0.20$ ( $\mathrm{t}$-test, $\mathrm{p}<0.05)$. The PFRI scores in the tested bridge group (score 4-5 high risk $=46$, $66 \%$, score 3 moderate risk $=36,68 \%$, and score $1-2$ low risk $=16$, $66 \%$ ) were higher than in the control healthy teeth group (score $4-5=20 \%$, score $3=30 \%$ and score $1-2=50 \%$ ). The mean PFRI was 3, 76 with SD 1, 20 on the test side and 2, 9 with SD 1, 14 on the control side. The mean percentage of reaccumulated plaque was at mesiobuccally and distobuccally sides of the first and second upper molars. The correlation between the value of the PFRI score and plaque $\mathrm{pH}$ was $\mathrm{r}=-0,01$ which showed weak negative relationship. Patients with FDP in high risk group (score 4-5) showed faster plaque forming at the restoration area, then opposite healthy side and need more frequent oral hygiene measures than slow plaque formers (score 1-2). The higher values of PFRI on the restoration site are slightly correlated with lower plaque $\mathrm{pH}$ values. These findings suggest that it is important to improve oral hygiene habits in patients with FDP in order to decrease the plaque index and cariogenic bacteria in the perspective of secondary caries prevention.

57

\section{Does the Restoration Failure Compromise the Primary Tooth Longevity? ART vs HT 2-yr RCT}

\author{
M.P. Araujo*a, I.C. Olegário ${ }^{\text {a }}$ D. Hesse ${ }^{\text {b }}$, N.P. Innes ${ }^{c}$, \\ C.C. Bonifácio ${ }^{\mathrm{b}}$, D.P. Raggio ${ }^{\mathrm{a}}$ \\ mariana.pinheiro.araujo@usp.br \\ ${ }^{a}$ Faculdade de Odontologia USP, São Paulo, Brazi; ${ }^{\text {b ACTA, }}$ \\ Amsterdam, The Netherlands; ' University of Dundee, United \\ Kingdom
}

The objective of this study is to assess the success of two restorative approaches, Atraumatic Restorative Treatment (ART) and the Hall Technique (HT) in occluso-proximal carious lesions in primary molars. After local Ethical Board approval (\#1.293.935) and ClinicalTrials.gov registration (NCT02569047), children aged 5-10 yrs $(\mathrm{n}=131)$ with primary molar occluso-proximal dentin lesions were recruited (Tietê, Brazil). One tooth per child was randomly allocated to ART (Equia Forte, GC Corp.) or HT (stainless steel crown). Evaluations were performed after 1, 6, 12, 18 and 24 months. Overall treatment "success" was absence of Major and Minor Failures. Minor Failures were restoration/crown defect, not interfering with tooth health and Major Failures were signs/symptoms of irreversible pulp damage, fistula/abscess, tooth fracture or irreparable failures. Kaplan-Meier survival analysis and log rank tests were carried out, while Cox regression test investigated associations between success and clinical variables $(\alpha=5 \%)$. Outcomes for 2 years are reported. The overall treatment success rate was $62.19 \%(\mathrm{HT}=93.4 \%$ and $\mathrm{ART}=36.1 \%)$ after 24 months $(\mathrm{HR}=$ $0.05 ; \mathrm{p}<0.001, \mathrm{CI}=0.01$ to 0.22 ). There were no Major Failures in $94.61 \%$ of the treated teeth $(\mathrm{HT}=98.44 \%$ and $\mathrm{ART}=91.46 \%)$ and no statistically significant difference between the groups $(\mathrm{HR}=$ $0.26 ; \mathrm{p}=0.23 ; \mathrm{CI}=0.029$ to 2.35 ). In primary molars with occlusoproximal cavities, HT shows better overall treatment success than ART after 2 yrs. Although there is a higher percentage of minor failures in ART group, the resulting 7\% difference in major failures, at 2 years, is similar and not significant.

This study has the support from FAPESP (\#2015/18098-2). 


\section{8}

\section{Sealing of Occlusal Dentinal Caries Lesions in Primary Molars: A 12-Month Follow-Up RCT}

N.M. Santos*a, C.S. Sartia , D.B. Gouvêa ${ }^{\text {a }}$, C.B. Azevedo ${ }^{\text {a }}$, J. Toniolo ${ }^{\text {, }}$, S.C. Leal ${ }^{\mathrm{b}}$, L. Casagrande ${ }^{\mathrm{a}}$, J.A. Rodrigues ${ }^{\mathrm{a}}$

nicolemarchioros@googlemail.com

aSchool of Dentistry, Federal University of Rio Grande do Sul, Porto Alegre, Brazil; 'bSchool of Dentistry, Federal University of Brasília, Brasília, Brazil

The aim of this RCT was to evaluate the efficacy of caries sealing (CS) in occlusal surfaces of primary molars compared to restoration (R) after selective caries removal. Thirty-two children aged 3 to 9 years old $(5.8 \pm 1.4$ years $)$ with high caries experience (mean dmf- $\mathrm{t}=8 \pm 3.2$ ) were included and 59 primary molars with dentinal lesions were selected and randomly divided into two groups (CS and R): lesions included in the CS group were sealed with lighted-cured glass ionomer cement (GC Fuji II LC capsule, GC Corporation - Tokyo, Japan) and in the R group were restored with the same material after selective caries removal technique. Clinical evaluation was performed based on modified USPHS criteria. Kaplan-Meier survival analysis was used to estimate the longevity of restorations and multivariate Cox regression with shared frailty was used to assess risk factors (treatment, age, gender, dmf$t$, teeth, location and lesion depth and extent). A total of 27 CS and $32 \mathrm{R}$ treatments were analyzed. The 12 -month overall survival rate reached $51.08 \%$. In the CS group the survival rate was $36.35 \%$ and in the R group $61.16 \%(\mathrm{p}<0.001)$. Patients in the CS group had more failures $(\mathrm{p}=0.02)$ than those in the $\mathrm{R}$ group (hazard ratio $0.20,95 \%$ CI: $0.04 ; 0.85)$. In conclusion, sealing caries-affected dentin using light-cured glass ionomer was more prone to failure compared to restorative treatment after selective caries removal in high caries experience children within 1 year.

\section{9 \\ Changes in Adolescent's Oral Health Status: Responsiveness of The Child Perception Questionnaire 11-14}

N.R. Aimée*a, A.J. van Wijk ${ }^{\mathrm{b}}$, M.M. Varjão a , A.B.Paes ${ }^{\mathrm{a}}$, J. Lourençoc, H.D. Mestrinho a , J.C. Carvalho ${ }^{\mathrm{d}}$

nicole.aimeerodrigues@gmail.com

aFaculty of Health Sciences, University of Brasília, Brasília, Brazil; ${ }^{b}$ Department of SocialDentistry, Academic Centre for Dentistry Amsterdam (ACTA), University ofAmsterdam and Vrije Universiteit Amsterdam, Amsterdam, The Netherlands; 'Health Departmentof the Federal District of Brazil, Brasilia, Brazil; ${ }^{d}$ Faculty of Medicine and Dentistry, CatholicUniversity of Louvain, Louvain, Belgium

Responsiveness refers to the sensitivity of an instrument to detect minimal but important clinical changes. This study assessed the responsiveness of the Child Perception Questionnaire (CPQ 11-14) to detect changes in oral-health-related quality of life
(OHRQoL) and oral health status of adolescents enrolled in a treatment program. The hypothesis tested was that improvement in OHRQoL would be observed after 1 year. The study was a controlled clinical trial with pre-test/post-test design. A total of 618 10-15 year olds were enrolled at baseline and 560 after 1 year. The test-retest of the CPQ 11-14 in 5\% of the sample had interclass correlation values of 0.63 and 0.70 . Reliability of the clinical and radiographic assessments of dental status showed kappa values between 0.79-0.91. At the baseline examination 374 adolescents needed only non-operative treatment (control group) whereas 274 needed non-operative and operative treatments (test group). After 1 year the adolescents were regrouped according their treatment needs fulfilled in Group 1 ( $\mathrm{n}=363$, control group) received nonoperative treatment; Group $2(\mathrm{n}=98)$ received both non- and operative treatments; Group $3(n=99)$ only received non-operative treatment but needed also operative treatment. The CPQ 11-14 total and subscale mean change scores by the global transition judgment indicated overall improvement of OHRQoL. There were significant differences of the total ( $p<0.001$, ANOVA) and subscale $(\mathrm{p}<0.005)$ mean changes scores within all five categories of global transition judgment. The hypothesis tested was accepted. The effect of the program for the treatment groups was significant $(\mathrm{p}=0.049$, ANCOVA). Group 3 had the smallest improvement, significantly smaller than Group $1(\mathrm{p}=0.014)$. In conclusion the CPQ 11-14 was sensitive enough to detect changes in OHRQoL and oral health status of adolescents after 1 year treatment. This finding supports the use of OHRQoL instrument to measure treatment program outcome.

Funded by the Brazilian National Council for Scientific and Technological Development (CNPq).

\section{0}

\section{Can Saliva Secretion Affect Oral Malodour and Oral Health-Related Quality of Life?}

\author{
Amin Homayouni*a, Behzod Tashbayeva, Shermin Rusthen", \\ Lene Hystad Hove ${ }^{\mathrm{b}}$, Morten Rykke ${ }^{\mathrm{b}}$, Preet Bano Singh ${ }^{\mathrm{a}}$, \\ Bente Brokstad Herlofson a, Janicke Liaaen Jensen ${ }^{a}$, Alix Young ${ }^{b}$ \\ amin.homayouni@odont.uio.no \\ aDepartment of Oral Surgery and Oral Medicine, Faculty of

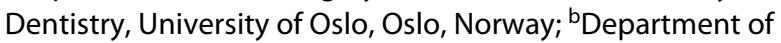 \\ Cariology and Gerodontology, Faculty of Dentistry, University \\ of Oslo, Oslo, Norway
}

The aim of this study was to investigate the relationship between saliva secretion, halitosis and oral health-related quality of life (HRQoL). Informed consent was obtained from 65 participants including 24 patients with primary Sjögren's Syndrome (pSS, $53 \pm 12 y$ ), 17 patients with dry eyes and dry mouth not fulfilling the classification for pSS (non-SS-sicca, $53 \pm 11 \mathrm{y}$ ), and 24 healthy controls ( $49 \pm 11 \mathrm{y}$ ). Unstimulated (UWS) and stimulated (SWS) whole saliva secretion rates were recorded as low (hyposalivation: SWS $<0.7 \mathrm{ml} / \mathrm{min}$, UWS $<0.1 \mathrm{ml} / \mathrm{min}$ ) or normal (SWS $>0.7 \mathrm{ml} / \mathrm{min}$, UWS $>0.1 \mathrm{ml} / \mathrm{min}$ ). Volatile sulfur compounds (VSC: hydrogen sulphide- $\mathrm{H}_{2} \mathrm{~S}$, methylmercaptan- $\mathrm{CH}_{3} \mathrm{SH}$ ) in mouth air were measured by gas chromatography. HRQoL was assessed using the Oral Health Impact Profile-14 (OHIP-14). Mann- 
Whitney test was used in data analysis $(\mathrm{p}<0.05)$. In general, for UWS, subjects with hyposalivation had significantly higher mean $\mathrm{CH}_{3} \mathrm{SH}$-levels than those with normal salivation $(27.3 \pm 39.3$ vs $9.9 \pm 13.6 \mathrm{ppb}$ ). For SWS, subjects with hyposalivation had significantly higher mean $\mathrm{H}_{2} \mathrm{~S}$-levels $(184.5 \pm 464.8$ vs $43.9 \pm 64.1$ $\mathrm{ppb})$ and $\mathrm{CH}_{3} \mathrm{SH}$-levels (27.2 \pm 37.4 vs $\left.6.5 \pm 8.9 \mathrm{ppb}\right)$. Within the non-SS patient group, those with hyposalivation had significantly higher $\mathrm{H}_{2} \mathrm{~S}$-levels than those with normal salivation. In general, there were no significant differences in OHIP-scores based on VSC levels. However, within the non-SS group, those with high $\mathrm{H}_{2} \mathrm{~S}$ levels $(>112 \mathrm{ppb})$ reported significantly more often that they had "difficulties relaxing due to oral problems", and that "life has been less satisfactory due to oral problems", than those with low $\mathrm{H}_{2} \mathrm{~S}$ levels ( $<112 \mathrm{ppb})$. In conclusion, hyposalivation was generally associated with increased levels of oral VSC, indicating oral malodour. Interestingly, the patients with dry eyes and dry mouth who did not fulfill the classification for pSS seemed to be more affected in their daily life than the patients who fulfilled the criteria.

Funded by a Research grant from the University of Oslo.

\section{1 \\ ICDAS II vs. Intraoral Camera in Early Occlusal Caries Detection: A Pilot Study \\ D. Corridore*a, M. Capocci ${ }^{\mathrm{a}}$, F. Rinaldo ${ }^{\mathrm{a}}$, F. Cocco ${ }^{\mathrm{b}}$, F. Guerra ${ }^{\mathrm{a}}$, G.M. Nardi ${ }^{\text {, L. Ottolenghi }}{ }^{\mathrm{a}}$ \\ denise.corridore@uniroma1.it \\ aDepartment of Oral and Maxillofacial Science "Sapienza" University of Rome, Rome, Italy; 'bepartment of Surgery, Microsurgery and Medical Sciences - School of Dentistry, University of Sassari, Sassari, Italy}

The aim of this in vivo study was to compare two performances of ICDAS II and Intraoral camera for the detection of pit and fissures' early caries lesions. Permanent premolars and molars were selected and evaluated in this in vivo study at the "Sapienza" University of Rome. Two blind operators inspected the occlusal surfaces of each tooth and the first one assigned an ICDAS II code (training was conducted by a senior ICDAS trainer in dental caries trained by a core member of the ICDAS Coordinating commitee), while the second one assessed the VistaCam score by using VistaCam ix Proof, Durr Dental. Level of agreement between the two procedures was expressed by using Cohen's/Fleiss' kappa statistic. 653 permanent teeth (306 premolars and 347 molars) in 13-30 years old patients were evaluated in this in vivo study. $27.2 \%$ of the assessed teeth was ICDAS II code $0,29.9 \%$ code $1,19.9 \%$ code 2 , $19.7 \%$ code 3 , 3.3\% code 4 . VistaCam assessed in $26.1 \%$ sound enamel, $50.3 \%$ initial enamel demineralization, $23.3 \%$ deep enamel decay, $0.3 \%$ dentin caries. The weighted kappa test showed coefficients ranging from 0.529 to 0.631 with an estimate of 0.582 . These results showed a moderate agreement between the two methods. Overall, our data suggest that the agreement between the two procedues is moderately reliable. Future randomized study will better define the role of VistaCam. Until then, combined usage of ICDAS II and fluorescence-based cameras should continue to be verified to define the standard of care.

\section{2 \\ Video-Observed Randomised Intervention Study: Improvement of Flossing Skills Through Video- Instruction versus Individualised Hands-On Training}

S.-C. Schmidt*a , N. Schlueter ${ }^{\mathrm{b}}$, C. Ganss $^{\mathrm{a}}$

sophie-charlotte.schmidt@dentist.med.uni-giessen.de aDepartment of Conservative and Preventive Dentistry, Dental Clinic, Justus-Liebig-University, Giessen, Germany; ${ }^{b}$ Division for Cariology, Department of Operative Dentistry and Periodontology, Center for Dental Medicine, Medical Center - University of Freiburg, Faculty of Medicine, University of Freiburg, Freiburg im Breisgau, Germany

Flossing correctly is challenging, so efficient instruction is crucial for its performance. To achieve this, observational data would be an expedient help. However, almost no observational data are available on how proper flossing is implemented. The aim was to assess flossing skills and their improvement after two different types of instruction (video-instruction versus video-instruction plus individualised hands-on training).

A random selection of students ( $24.3 \pm 3.0$ years) was included, not being involved in human medicine and/or dentistry was mandatory. Group $1(\mathrm{n}=49)$ watched a video demonstrating correct flossing of the whole dentition followed by an individualised hands-on training, group $2(\mathrm{n}=48)$ watched the video only. At baseline, after instruction (T2) and a third time (7-day intervals; T3) subjects flossed while being video-filmed through a mirror. Videos were analysed with the observation software INTERACT regarding reached proximal spaces (median; min,max) and flossing technique (vertical strokes, proper handling and cleaning mesial and distal surfaces). Statistics: Wilcoxon-test, McNemar-test. At baseline, $18.6 \%$ of the students used the right flossing technique (vertical strokes) to clean the tooth surface, $18.6 \%$ flossed the proximal spaces in whole (mesial and distal separately). 9.6\% handled the floss correctly. The median number of reached proximal spaces was 22 (1.25). At T2 technique improved significantly: $64.2 \%$ used vertical strokes, $88.4 \%$ flossed the proximal spaces in whole and $91.6 \%$ handled the floss correctly ( $\mathrm{p} \leq 0.001$ each). Reached proximal spaces increased significantly ( $\mathrm{p} \leq 0.001)$ to $23(2.26)$. At T3 no further enhancement occurred with all parameters. There were no significant differences between the two instruction types. To sum it up, single video instruction led to highly enhanced flossing skills especially with respect to the flossing technique. Handson instruction did not further improve the results.

Funded by Justus-Liebig-University, Giessen. 


\section{3}

\section{Orthodontic White Spot Lesions (WSLs) Can Affect Patients' Aesthetic Satisfaction? A Spectrophotometric Approach}

\author{
M. Mazur*, F. Rinaldo, D. Salvi, A. Ndokaj, L. Ottolenghi,
}

G.M. Nardi, F. Guerra

marta.mazur@uniroma1.it

Department of Oral and Maxillofacial Sciences, "Sapienza"

University of Rome, Rome, Italy

The aim of this in vivo study was to evaluate the colourimetric changes between sound enamel and post orthodontic WSLs and to correlate these findings with human eye colour perception threshold using spectrophotometry. Experimental approach Seventy-six permanent teeth with post orthodontic WSLs in 12-16 years old patients were evaluated in this in vivo study at "Sapienza"University of Rome. The spectrophotometric differences in colour $\left(\Delta \mathrm{L}^{*}, \Delta \mathrm{a}^{*}\right.$, $\Delta \mathrm{b}^{*}$; Color Difference $\Delta \mathrm{E} 00$; Whiteness Index $\left.\Delta \mathrm{WIO}\right)$ between sound enamel and clinically detected WSLs in each tooth were calculated. A correlation between Acceptability Threshold (AT = 3.3) and the calculated CIEDE2000 colour difference was performed. The $\mathrm{L}^{*}$ value is measure of the lightness ranging from 0 (black) to 100 (white), the $\mathrm{a}^{*}$ value of redness/greenness $\left(\mathrm{a}^{*}><0\right)$, and the $\mathrm{b}^{*}$ value of yellowness/blueness $\left(b^{*}><0\right)$. The colour change between sound and affected enamel for each tooth was defined by the CIEDE2000 colour space $(\Delta \mathrm{E} 00)$. WIO Index is the most up to date and appropriate index to measure tooth whiteness. Spectrophotometric differences in colour between sound enamel and clinically detected post orthodontic WSLs show a decrease in $\Delta \mathrm{L}^{*}(-1.06)$ and $\Delta$ WIO $(-11.88)$ and an increase in $\Delta \mathrm{a}^{*}(0.43)$ and $\Delta \mathrm{b}^{*}(2.94)$. Overall colour change $\Delta \mathrm{E}$ (4.38) was always higher than the Acceptability Threshold. In conclusion white spot lesions due to orthodontic fixed therapy affect colourimetric properties of teeth and the amount of the calculated colour difference is on average always higher than 3.3, indicating a detectable and aesthetically unacceptable, colour difference.

\section{4}

\section{Effects of an Oral Care Programme for Adults-Evaluation After 15 Years}

C. Witecy*a, M. Heins ${ }^{\mathrm{a}}$, N. Schlueter ${ }^{\mathrm{ab}}$, C. Ganss ${ }^{\mathrm{a}}$

Corinna.Witecy@dentist.med.uni-giessen.de

aDepartment of Conservative and Preventive Dentistry,

Dental Clinic of the Justus-Liebig-University Giessen, Giessen,

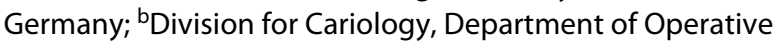
Dentistry and Periodontology, Center for Dental Medicine, Medical Center - University of Freiburg, Faculty of Medicine, University of Freiburg, Freiburg im Breisgau, Germany

Effects of oral hygiene education are mostly investigated in children/adolescents, but little is known about effects of prevention programmes in adults. The present study is a retrospective analysis of our Oral Care Programme (OCP) aiming to evaluate its effect on oral hygiene (API) and gingivitis (PBI) scores. The OCP consisted of four modules: oral hygiene instruction, nutrition counselling, fluoridation/re-motivation, and re-instruction when needed. Patients were not recalled but organised appointments themselves. Data were extracted from records (1999-2014) including 1665 patients. Age, gender, type/date of modules, and API/PBI were recorded. Patients were stratified in good (API $\leq 0.05 / \mathrm{PBI}$ $\leq 0.03$ ), fair (API $>0.5$ to $\leq 0.75 / \mathrm{PBI}>0.03$ to $\leq 0.14$ ) and poor oral hygiene (API $>0.75 / \mathrm{PBI}>0.14$ ) at baseline. Time periods were stratified in 5-year-intervals and instruction intervals in $\leq 4$ weeks, $4 \leq 8$ weeks, 8 weeks $\leq 1$ year and $>1$ year. API/PBI is given as median (min;max). Statistics: Wilcoxon-test, Mann-Whitney-U-test, cross-tabulations, chi-square-test. Overall, $60.2 \%$ attended the OCP once, $19.1 \%$ twice, $20.7 \%$ three times or more (max. 36 ). At the first visit API/PBI was $0.68(0 ; 1) / 0.08(0 ; 1)$ resp. which decreased at visit two $(0.62(0 ; 1) / 0.06(0 ; 1)$ resp.; $\mathrm{p} \leq 0.001$ each $)$ with no further decrease over next visits. Patients with poor oral hygiene improved at the next visit, but those with good oral hygiene worsened ( $\mathrm{p} \leq 0.001$ each). API and PBI improved ( $\mathrm{p} \leq 0.001$ each) with shorter ( $\leq 4$ or $\leq 8$ weeks) compared to longer intervals (8 weeks $\leq 1$ year and $>1$ year). User behaviour changed significantly over the 5-year-intervals: earlier, more and more different modules were attended; later, patients attended predominantly the oral hygiene instruction module at longer intervals. The best effect of the OCP was in patients with poor oral hygiene and when shorter instruction intervals were used.

Funded by Justus-Liebig-University, Giessen.

65

\section{Evaluation of a New QLF Plaque Scoring Algorithm,} SHS

D.A.Kahharova*a, B.O. Khudanov a , B.I. Kimb ${ }^{\text {, E.S. Lee }}$, E. de Josselin de Jong ${ }^{\mathrm{b}, \mathrm{c}}$

donokahharova@gmail.com

aPreventive and Pediatric Dentistry Department of Tashkent

State Dental Institute, Tashkent, Uzbekistan; ' ${ }^{\circ}$ Department of

Preventive Dentistry \& Public Oral Health, Yonsei University,

Seoul, South Korea; 'Inspektor Research Systems B.V,

Amsterdam, The Netherlands

Qraycam, based on QLF technology, is a tool to identify early intra-oral anomalies. To capture and quantify the plaque tooth coverage software Qray (v1.32 Inspektor Research Systems) was used. The software comprised of a plaque analysis tool called Simple Hygiene Score (SHS), which assessed the plaque coverage and severity in a score from 0 to 5 . The aim was to compare the SHS with the conventional Quigley-Hein plaque index (QHI). Fluorescent frontal images of the upper and lower anterior teeth were made of 77 dental students at the age of 18-26. Plaque was disclosed using a cotton swab with a two-tone dye solution (Mira2-Ton; Hager \& Werken, Duisburg, Germany). The dye solution was gently applied on the vestibular tooth surfaces. The dye induced two colors: red indicating young plaque and blue indicating young and mature plaque. The QHI (0 to 5) was scored based on the red and blue dyed areas combined $(\mathrm{QHIR}+\mathrm{B})$, and on the blue areas only (QHIB) for each individual tooth surface from element $\# 13$ to $\# 23$, and from \#33 to \#43. The SHS values were plotted 
against the respective QHI values and linear regression analysis was applied. For SHS vs QHIB the slope was 1.45 and correlation $0.71(\mathrm{p}<0.001)$ indicating a moderate to strong relationship. For SHS vs QHIR+B the slope was 0.93 and correlation 0.58 ( $\mathrm{p}<$ $0.001)$, moderate. It was concluded that the Simple Hygiene Score (SHS) correlated moderate to strong with the Blue Quigley-Hein Index (QHIB), mature plaque. The SHS correlation with the Red+ Blue Quigley-Hein Index $(\mathrm{QHIR}+\mathrm{B})$, young plaque, was moderate. SHS might be useful to quantitatively assess mature plaque coverage and severity.

Funded by AIOBIO company, Seoul, South Korea

\section{6 \\ Examiners' Agreement and Reliability for the Measurement of Caries Activity with Bio-Luminescent Marker Images and Ex-Vivo}

S. Martignon ${ }^{\mathrm{a}, \mathrm{b}}$, A. Cortés*b , N.B. Pitts ${ }^{\mathrm{a}}$, K.R. Ekstrand ${ }^{\mathrm{c}}$, V. Avila ${ }^{\mathrm{b}}$, G.A. Castiblanco ${ }^{\text {b }}$ L.F. Gamboa ${ }^{\text {b }}$, D. F. Cabrera ${ }^{\mathrm{b}}$

cortesandrea@unbosque.edu.co

aDental Innovation and Translation Centre, Kings College London, London, UK; 'bNICA - Caries Research Unit, Research Vice-rectory, Universidad El Bosque, Bogotá, Colombia; 'Cariology \& Endodontics, Department of Odontology, Faculty of Health and Medical Sciences, University of Copenhagen, Copenhagen, Denmark

This study aimed to establish the agreement (Perfect Agreement: PA) and reliability (weighted Kappa values: K) of initial caries (ICDAS 1-2) activity assessments with bio-luminescent marker images $\left(\right.$ Calcivis $\left.{ }^{\circledR}\right)$, and clinically ex-vivo, according to dentition and surface location. Following IRB approval and with patients/ caregivers' signed consents, 44 subjects with teeth scheduled for extraction were enrolled. An external trained examiner selected sound/initial-caries specific sites on teeth surfaces. After extraction, stereomicroscopic photographs plus bench-top-prototype Calcivis ${ }^{\circledR}$-system bio-luminescent marker images were obtained and organized in slides indicating assessment sites plus fluorosis, plaque and gingival-bleeding information from clinical exam. Four-trained examiners independently assessed (and re-assessed one week later) randomly organized images. One week later one examiner assessed (and re-assessed four days later) activity clinically ex-vivo with randomly organized sites. In total 78 teeth were included $(\mathrm{n}=35$ primary, $\mathrm{n}=43$ permanent; $\mathrm{n}=51$ posterior, $\mathrm{n}=$ 27 incisor) with 350 sites ( $\mathrm{n}=164$ primary, $\mathrm{n}=186$ permanent teeth; $\mathrm{n}=239$ smooth surfaces, $\mathrm{n}=111$ pit-and-fissure system). Most (84.8\%) had mild-moderate fluorosis (TFI). Examiners categorized in bio-luminiscent marker images a mean of $290.5 \pm 17.2$ sites as sound/inactive lesions and $59.5 \pm 17.2$ as active lesions. Mean activity inter-examiner data: $\mathrm{PA}=83.7 \%(77.8 \%-89.7 \%)$, $\mathrm{K}=0.66 \pm 0.10$. Correspondent data regarding dentition: Primary teeth: $\mathrm{PA}=86.32 \%(79.9 \%-92.6 \%), \mathrm{K}=0.70 \pm 0.12$; Permanent teeth: $\mathrm{PA}=81.2 \%(75.8 \%-87.4 \%) ; \mathrm{K}=0.62 \pm 0.09$. Regarding surface location: Smooth surfaces: PA $=83.7 \%(79.1 \%-88.4 \%)$ vs. Pitand-fissure system: $\mathrm{PA}=84.1 \%(75.0 \%-93.1 \%)$, and $\mathrm{K}=0.67 \pm$ 0.08 vs. $\mathrm{K}=0.62 \pm 0.16$, respectively. Intra-reproducibility PA: $77.4 \%-91.0 \%$ and $\mathrm{K}: 0.51-0.73$. Ex-vivo distribution of sites cor- responded to: Sound: $\mathrm{n}=228$, Initial-Inactive-Caries lesions: $\mathrm{n}=$ 52, and Initial-Active-Caries lesions: $\mathrm{n}=70$. Ex-vivo general activity intra-examiner were: $\mathrm{PA}=96.5 \%$ and $\mathrm{K}=0.89$. In conclusion the assessment of activity for caries initial lesions showed between examiners an almost Perfect Agreement and substantial weighted Kappa values in this teeth sample with a high prevalence of dental fluorosis.

This study was partially funded by Universidad El Bosque, Colombia; King's College London, UK; and University of Copenhagen, Denmark, and Calcivis ${ }^{\circledR}$.

\section{7 \\ Success Rates of Hall Crowns for Managing Carious Primary Molars: Retrospective Analysis \\ R. Midani*, C.H. Splieth, M. Mustafa Ali, J. Schmoeckel, M.S. Mourad, R.M. Santamaria \\ midanirama@gmail.com \\ Department of Preventive and Paediatric Dentistry University of Greifswald, Greifswald, Germany}

The aim of this retrospective study was to evaluate the clinical efficacy (minor/major failures) and survival rates (successful cases without any failures) of stainless steel crowns (SSCs) placed on primary molars using the Hall Technique (HT) at the Department of Preventive and Pediatric Dentistry at Greifswald University/ Germany. Patient records of children receiving SSCs placed according to the HT (with no tooth preparation or with minimal proximal slice, without caries removal), between 2013 and 2017 by 29 dentists were reviewed. For the analysis, only carious primary molars with sufficient documentation on clinical examinations at baseline assessing no clinical or radiographic evidence of pulp involvement (pulpitis/necrosis/abscess) and at least six months follow-up were included. Only one tooth per child was included for the analysis. Kaplan-Meier survival analyses with Mantel-Cox statistics were carried out. 143 Hall crowns performed in 2-10-yearolds (mean $6.05 \pm 1.9 \mathrm{yrs}$ ) with mean follow-up data of 22 months $( \pm 15.7)$ were included for analysis. In these 54 girls and 89 boys the mean d3mft was 6.38. Overall, $132(92.3 \%)$ crowns were successful, three $(2.1 \%)$ presented at least one minor failure (reversible pulpitis, crown lost, or secondary caries), and eight (5.6\%) a major failure (irreversible pulpitis or abscess). The majority of failures occurred during the first year (55\%) after crown placement. By comparing crowns performed without tooth preparation $(n=119)$ to crowns performed with proximal slice $(n=24)$, no differences were observed ( $\mathrm{p}=0.70, \mathrm{CI}=0.68$ to 0.83 ). The probability of surviving one year without having a minor/major failure was $94 \%$, and for three years $85 \%$. Survival rate and clinical efficacy of Hall crowns for asymptomatic carious primary molars in high caries risk children were high in a secondary care-based setting.

This study was funded by the Department of Preventive and Pediatric Dentistry, University of Greifswald. 


\section{8}

\section{Salivary Cortisol Response to Dental Treatment of Anxious Children with Early Childhood Caries}

\section{A. Osokina*, E. Maslak}

osoka_as@mail.ru, asrodionova@bk.ru

Pediatric Dentistry Department, Volgograd State Medical

University, Volgograd, Russia

The aim was to compare salivary cortisol levels (CL) of anxious children before and after dental treatment. The study was carried out in Volgograd Pediatric Dental Clinic with approval from the regional Ethics Committee. The parents' informed consents were obtained. 45 children aged 18-83 months with Early Childhood Caries (ECC) with negative or definitely negative behavior (according to Frankl scale) were included into the study. The children were randomly selected in 2 treatment groups: CT $(n=22)-$ conventional (drilling and filling) and NIT $(n=23)$ - non-invasive sealing cavitated lesions. Cases in each group were stratified into subgroups according to the children's age: CT1 $(n=11)$ and NT1
( $\mathrm{n}=12$ ) included $18-48$ months old children (mean age $26.3 \pm$ 9.1), CT2 $(\mathrm{n}=11)$ and NT2 $(\mathrm{n}=11)$ included $49-83$ months old children (mean age $58.3 \pm 10.5$ ). Unstimulated saliva was collected before and after dental treatment. The CL level in saliva was analyzed using immunoassay,"Hema", Russia. Wilcoxon test was used to compare the results, with significance at $\mathrm{p}$-value $<0.05$. In children aged 18-36 months the post-treatment CL was significantly higher than the pre-treatment level in both groups (CT1: $4.57 \mathrm{ng} / \mathrm{ml}$ and $7.37 \mathrm{ng} / \mathrm{ml}$ respectively, $\mathrm{p}=0.007$; NT1: $3.51 \mathrm{ng} /$ $\mathrm{ml}$ and $8.08 \mathrm{ng} / \mathrm{ml}$ respectively, $\mathrm{p}=0.0022)$. In children aged 49 83 months the post-treatment CL in NT2 was significantly lower than the pre-treatment level $(4.51 \mathrm{ng} / \mathrm{ml}$ and $2.56 \mathrm{ng} / \mathrm{ml} \mathrm{respec-}$ tively, $\mathrm{p}=0.005)$ and in CT2 pre- and post-treatment CL were similar $(1.21 \mathrm{ng} / \mathrm{ml}$ and $1.18 \mathrm{ng} / \mathrm{ml}$ respectively, $\mathrm{p}=0.043)$. The increase of CL after treatment in both group of the children aged 18-36 months demonstrated a disruptive behavior of young children during the dental treatment. The decrease of CL was only revealed after non-invasive treatment in the children aged 49-83 months.

Funded by Pediatric Dentistry Department, Volgograd State Medical University, Russia. 


\section{Sessions 7 and 8 Epidemiology}

\author{
69 \\ Prevalence of Hypersensitivity in Teeth Affected by \\ Molar-Incisor Hypomineralization (MIH) \\ F. Raposo*, E.N. Lia, A.C. Rodrigues, S.C. Leal \\ fe.raposo6@gmail.com \\ Department of Dentistry, Faculty of Health Science, University \\ of Brasília, Brasília, Brazil
}

This study aimed to investigate the prevalence of hypersensitivity in MIH-affected molars through a census that was carried out in 8-years-old schoolchildren. Examinations were conducted by one calibrated examiner, using the Nyvad criteria for caries' diagnosis and a new criterion for MIH assessment. For hypersensitivity assessment, all MIH-affected molars were evaluated. The none $\mathrm{MIH}$-affected molars were used as controls. Air blast reaction was measured using the Visual Analogue Scale (VAS) and the SCASS (Schiff Cold Air Sensitivity Scale) scale, while tactile hypersensitivity was scored using VAS only. Statistical analysis was performed using Kruskal-Wallis test followed by Dunn's multiple comparisons test for quantitative data. Chi-square was used for comparison of categoric data. 631 children were assessed, of whom 102 had MIH-affected teeth (16.1\%). Of these, $51.7 \%$ and $8.7 \%$ children presented enamel and dentin carious lesions, respectively. Regarding the number of teeth, 239 molars were MIH-affected (59.8\%), with $188(78.7 \%)$ classified as mild (opacities only), $20(8.4 \%)$ as moderate (post-eruptive enamel breakdown), and 31 (13\%) as severe (post-eruptive breakdown involving dentin). Hypersensitivity was not recorded in any control teeth, while the prevalence of hypersensitivity in MIH-affected molars was $34.7 \%$, being of low intensity and more prevalent in moderate $(51.6 \%)$ and severe cases $(55 \%)$ than in mild cases $(29.8 \%, p=0.008)$. An association between hypersensitivity and the presence of mild and moderate cases was observed, but not in severe cases, as $90 \%$ of $\mathrm{MIH}$-affected molars with post-eruptive breakdown involving dentin were affected by dentin carious lesions. In conclusion, hypersensitivity was significantly higher in $\mathrm{MIH}$-affected molars than in non-affected molars, being associated to MIH teeth presenting opacities and enamel breakdown.

65th ORCA Congress
This study was partially supported by National Council for Scientific and Technological Development (CNPq) and Coordination of Improvement of Higher Level Personnel (CAPES) from the Brazilian government, respectively, under the grants: 306852/2016 and 1752526.

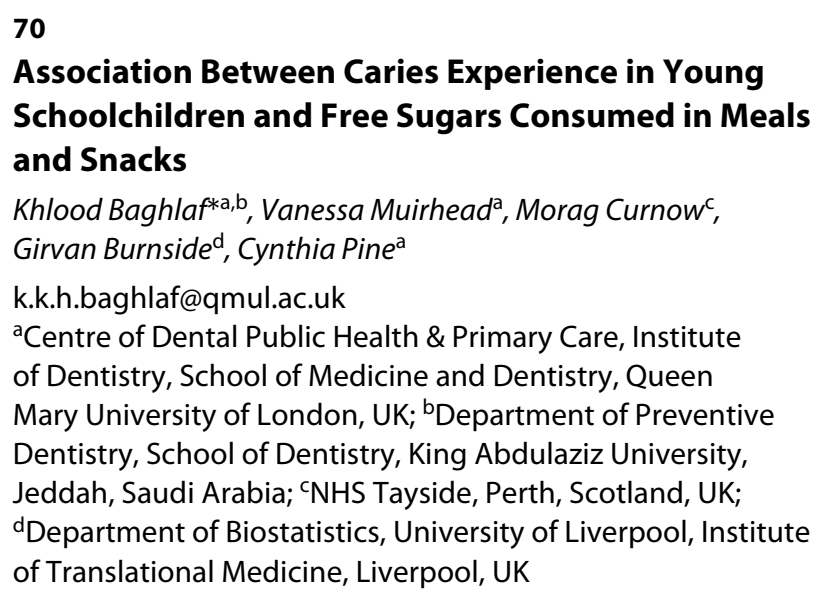

k.k.h.baghlaf@qmul.ac.uk

${ }^{a}$ Centre of Dental Public Health \& Primary Care, Institute of Dentistry, School of Medicine and Dentistry, Queen Mary University of London, UK; ${ }^{b}$ Department of Preventive Dentistry, School of Dentistry, King Abdulaziz University, Jeddah, Saudi Arabia; ' NHS Tayside, Perth, Scotland, UK; dDepartment of Biostatistics, University of Liverpool, Institute of Translational Medicine, Liverpool, UK

The aim was to examine any associations in five-six-year old children between caries experience $\left(\mathrm{d}_{3} \mathrm{mft}\right)$; untreated dental caries $\left(d_{3} t\right)$ and: their daily total free sugars (FS); average daily frequency of FS; FS consumed at meals and snacks. 149 children aged five-six years whose parents completed a 3-day diet diary. Children comprised a sub-group of participants within 25 primary schools in Tayside, Scotland of the Bedtime Brushing and Read Together to Sleep (BBaRTS) cluster randomized controlled trial at baseline. Dental examinations by calibrated dentists using the British Association for the Study of Community Dentistry (BASCD) criteria. Diaries were input into INTAKE24: an online dietary assessment tool. Outputs calculated: energy intake, total FS, frequency of FS. 143 of the 149 children who completed 3-day diet diaries had dental examinations: 80 girls and 63 boys: mean $\mathrm{d}_{3} \mathrm{mft} 0.8$ (s.d. $=1.6$ ); $\mathrm{d}_{3} \mathrm{t}=0.5$ (s.d. $=1.1$ ); $27 \%$ of children had caries experience. Mean daily FS consumption was $77.8 \mathrm{~g}(\mathrm{SD}=49.5)$. The highest intake 
of FS was at lunchtime $(18.9 \mathrm{~g})$ and the lowest recorded for bedtime snacks $(6.3 \mathrm{~g}) .123(86 \%)$ of children consumed more than the WHO recommended sugar intake of $10 \%$ of their total energy intake. The mean frequency of FS consumption was four times per day and was significantly correlated with FS total daily amount (Spearman rho $(\mathrm{rs})=0.5 \mathrm{p}<0.001)$. No correlation was found between $\mathrm{d}_{3} \mathrm{mft}$ and the frequency of FS consumption or between $\mathrm{d}_{3} \mathrm{mft}$ and $\mathrm{FS}$ total $\left.(\mathrm{rs})=0.1 \mathrm{p}=0.2\right)$. In conclusion no significant associations were found between FS frequency; FS amount and caries experience in this population of children who consumed free sugars mainly at meal times.

This study was funded by an unrestricted grant from GlaxoSmithKline.

\section{1 \\ Diet and Caries Experience in the Netherlands Among Adults from Western or Non Western Cultural Background \\ D.Y. Tang ${ }^{\mathrm{a}, \mathrm{b}}$, M.H. van der Veen*a \\ Elise.Tang@inholland.nl \\ aOral Hygiene, Inholland University of Applied Sciences, Amsterdam, The Netherlands; ${ }^{b}$ Clinical Epidemiology, AMC, Amsterdam, The Netherlands}

This study investigated the effect of socioeconomic status (SES) and cultural background, Western (W) or Non Western (NW); i.e. with a migration background from one of the countries in Africa, Latin America or Asia (excluding Indonesia and Japan) or Turkey) on dietary pattern and how these may affect caries in a sample of adults in the Netherlands. Subjects in the study were aged 30 to 60 years, enrolled at a dental practice with recent data on DMFT $(<1 \mathrm{yr})$. All subjects gave informed consent. Data on demographics, including SES and cultural background, as well as dietary pattern (intake of fruit, vegetables, water and snacking behaviour) were asked by means of a questionnaire and linked to DMFT data recorded from patient records. Respondents were $123 \mathrm{~W}$ Subjects (47.4 yr $\pm 9.1 \mathrm{yr} ; 49.6 \%$ male; $60.2 \%$ high SES) and $30 \mathrm{NW}$ subjects (45.5 yr $\pm 10.0 \mathrm{yr} ; 36.7 \%$ male, $50 \%$ high SES). No differences were found between W or NW background for dietary intake ( $p>0.05)$. Differences were found between SES groups for vegetable consumption among W subjects $(\mathrm{p}=0.003$, Kruskal Wallis, High SES $>$ Low or Middle SES) and snacking frequency $(p=0.043$, Kruskal Wallis, Middle SES<low or high SES). Among NW subjects no differences in dietary pattern were observed between SES groups. DMFT and number of fillings were significantly higher in W subjects than NW subjects (DMFT:6.1 \pm 5.2 and $3.9 \pm 4.6, \mathrm{p}=0.039$; $\mathrm{D}: 0.2 \pm 0.7$ and $0.1 \pm 0.3, \mathrm{p}=0.530 ; \mathrm{M}: 0.2 \pm 1.5$ and $0.4 \pm 1.2, \mathrm{p}=$ $0.521 ; \mathrm{F}: 5.7 \pm 4.9$ and $3.4 \pm 4.4, \mathrm{p}=0.022)$. Among $\mathrm{W}$ subjects DMFT and number of fillings differed among SES groups ( $\mathrm{p}=$ 0.013 respectively $\mathrm{p}=0.029$, One Way ANOVA, Low $\mathrm{SES}>$ Middle SES and High SES=Middle or Low SES). No effect of SES was found in NW subjects. The DMFT and number of fillings among subjects with Non Western background is lower than that of subjects with Western background. No clear cut relation with diet or SES is seen.

\section{2 \\ Caries Development in 12 Years Old Children in Germany: Projection vs. Reality}

R. Basner*, M.S. Mourad, M. Mustafa Ali, A. Basta, A. Al-Ani, C.H. Splieth

roger.basner@uni-greifswald.de

Department of Preventive and Pediatric Dentistry, University

of Greifswald, Greifswald, Germany

The aim of this study was to check the accuracy of a dental caries projection model for 12 years old children (Derkach 2013) which classified this group according to the educational level and the socioeconomic status. Based on the work of Derkach (2013), in which the caries indices, extractions and restorations from epidemiological data (IDZ 1997-2005) were projected to 2030 for a caries risk group (30\%, low SES) and the main group (70\%, moderate/ high SES), a comparison with the recent data [IDZ 2016] was made. Caries prevalence in 12 years old children permanent teeth has been showing a saturation function for the DMFT for years, which was described by Derkach in 2013 using the formula $y=$ $8 \mathrm{E}+57 \mathrm{x}^{-12.68}$ for the main group. In this model, a DMFT value of 0.24 was predicted for 2015 which was slightly lower than the real data (DMFT 0.35) from IDZ study. For the low SES group the formula of $y=7 \mathrm{E}+38 \mathrm{x}^{-8.498}$ resulted with a predicted DMFT value of 0.4 for 2015, while in the IDZ study recorded a higher DMFT value was of 0.7 DMFT. The predicted mean value for all 12-year-olds would have been 0.29 DMFT which is also lower than the actual 0.5 DMFT from 2015. In conclusion, the caries decline in 12-yearolds in Germany seems to reach a saturation point on a low, but slightly higher level and polarization than a previous projection of prior data would suggest.

\section{3 \\ National Oral Health Survey on Refugees in Germany 2016/2017 (children and adolescents) \\ C.H. Splieth*, M. Takriti, J. Schmoeckel, M. Alkilzy, A. Al-Ani \\ splieth@uni-greifswald.de \\ Department of Preventive and Pediatric Dentistry, University of Greifswald, Greifswald, Germany}

Due to the marked increase in refugees and asylum seekers and a lack of health data, a multicentre survey on the oral health status was initiated in Germany. 256 children and adolescents from age 3 to 17 were examined in different age categories in 2016/17 in 10 registration institutions well distributed over Germany by two calibrated dentists. They came mostly from Syria (30.8\%), Iraq (14.4\%), and Afghanistan (15.6\%). The examination included the $\mathrm{dmft} / \mathrm{DMFT}$ index according to WHO criteria [2013] and the pufa/PUFA index [Monse et al. 2009]. Caries experience increased continuously in the primary dentition from 3-year-olds $(2.54 \pm$ $3.6 \mathrm{dt} ; 2.62 \pm 3.6 \mathrm{dmft})$ to $6-7$-year-olds $(4.21 \pm 3.4 \mathrm{dt} 4.21 \pm 3.4$; $5.22 \pm 3.4 \mathrm{dmft}$ ). Hardly $50 \%$ of the 3 -year-olds and $7 \%$ of the 6-7-year-olds had never exhibited caries on a defect level in the primary dentition $(\mathrm{dmft}=0)$. Mean DMFT values in 12-year-olds 
were $2.00( \pm 1.9$; DT $1.12 \pm 1.3 ; 12 \% \mathrm{dmft} / \mathrm{DMFT}=0$; comparison Germany 0.5 DMFT, 81\% DMFT $=0$ [IDZ 2016]) and increase for 13 -17-year-olds to $2.87( \pm 2.7$; DT $1.93 \pm 2.01 ; 23 \%$ DMFT $=0$; mean age $14.9 \pm 1.33$ ). The pufa index was especially high in the primary dentition (6-7-year-olds $0.86 \pm 1.38$ ) and less pronounced in the permanent dentition of adolescents (13-17-yearolds; PUFA $0.18 \pm 0.55)$. In conclusion children and adolescents who came to Germany recently as refugees and asylum seeker exhibit higher numbers of carious defects and total caries experience, and also more fistulas, abscesses etc. than the resident German population, especially in the primary dentition. For a successful integration, it would be of importance to close this prevention and treatment gap.

The study was funded by Deutsche Gesellschaft für Zahn-, Mund- und Kieferheilkunde, Bundeszahnärztekammer, Kassenzahnärztliche Bundesvereinigung and the Wrigley Company Foundation and was approved by the Ethics Committee of the University of Greifswald (BB021/17).

\section{4 \\ National Oral Health Survey on Refugees in Germany 2016/2017 (Adults)}

J. Schmoeckel*, A. Al-Ani, M. Takriti, M. Alkilzy, C.H. Splieth

julian.schmoeckel@uni-greifswald.de

Department of Preventive and Pediatric Dentistry, University

of Greifswald, Greifswald, Germany

Due to the marked increase in adult refugees and asylum seekers and a lack of health data, a multicentre survey on the oral health status was initiated in Germany. 283 adults aged 18 to 64 years were examined (18-34-year-olds: $\mathrm{n}=123$; 35-44-year-olds: $\mathrm{n}=87$; 45-64-year-olds: $\mathrm{n}=73$ ) in 2016/17 in 10 registration institutions well distributed over Germany by two calibrated dentists. Most adult refugee participants came from Middle Eastern $(n=111)$ and Asian countries $(n=100)$ and fewer came from Africa $(n=37)$ and Eastern Europe $(n=34)$. The examination included the DMFT index according to WHO criteria [2013] and the PUFA index [Monse et al. 2009]. Caries experience increased from 18-34-yearolds $(7.43 \pm 5.7$ DMFT; $3.72 \pm 3.0$ DT) over 35-44-year-olds $(10.55 \pm 7.1$ DMFT; $3.13 \pm 3.0$ DT, comparison Germany: 11.2 DMFT [IDZ 2016]) to 45-64-year-olds (3.64 \pm 4.1 DT; $14.92 \pm 7.7$ DMFT, comparison Germany: for 65-74-year-olds 17.7 DMFT [IDZ 2016]). Less than $5 \%$ of the adult refugees had a caries free dentition $(\mathrm{DMFT}=0)$. The proportion of participants with PUFA $>0$ and the mean PUFA value were almost constant for the different adult age groups (18-34-year-olds: 25\% PUFA $>0$, $\varnothing$ PUFA $0.40 \pm 0.8$; 35-44-year-olds: $23 \%$ PUFA $>0$, $\varnothing$ PUFA $0.37 \pm 0.9$; 45-64-year-olds: $31 \%$ PUFA $>0$, Ø PUFA 0.45 \pm 0.8 ). In 35-44-yearolds, African refugees had clearly lower caries experience than Middle Eastern, Eastern European and Asian refugees (3.5 DMFT $\pm 1.41,9.24 \pm 5.59,15.44 \pm 6.11$ and $11.65 \pm 8.93$, resp.). In conclusion adult refugees and asylum seekers who came to Germany recently exhibit higher numbers of carious defects, dental pain, fistulas and abscesses. With increasing age, similar overall DMFT values compared to the German residential population were found, but higher MT and DT components in refugees reveal a focus on extraction therapy and persistent higher treatment needs.

The study was funded by Deutsche Gesellschaft für Zahn-, Mund- und Kieferheilkunde, Bundeszahnärztekammer, Kassenzahnärztliche Bundesvereinigung and the Wrigley Company Foundation and was approved by the Ethics Committee of the University of Greifswald (BB021/17).

\section{5 \\ Caries Development in 35-44 Years Old Adults in Germany: Projection vs. Reality}

M.S. Mourad, M. Mustafa Ali, A. Basta, A. Al-Ani, C.H. Splieth

mhd.mourad@uni-greifswald.de

Department of Preventive and Pediatric Dentistry, University

of Greifswald, Greifswald, Germany

The aim of this study was to check the accuracy of a dental caries projection model for 35-44 years old adults (Derkach 2013) which classified this group according to the educational level and the socioeconomically status. Based on the work of Derkach (2013), in which the caries indices, extractions and restorations from epidemiological data (IDZ 1997-2005) were projected to 2030 for a caries risk group (30\%, low SES) and the main group (70\%, moderate/high SES), a comparison with the recent data [IDZ 2016] was made. For the first time in the 35-44-year-olds, the current IDZ study (2016) found a significant increase in healthy teeth $(2.5 \%)$. In this projection model the DMFT value was described using the formula $\mathrm{y}=2 \mathrm{E}+07 \mathrm{x}^{-1.315}$ for the main group. A DMFT value of 12.6 was predicted for 2015 which was higher than the real data (DMFT 10.8) from IDZ study. For the low SES group the formula of $y=33445 \mathrm{x}^{-0.726}$ resulted with a predicted DMFT value of 14.7 for 2015, while in the IDZ study recorded a lower DMFT value was of 12.4 DMFT. The predicted mean value for all 35-44-year-olds would have been 13.2 DMFT which is also higher than the actual 11.2 DMFT from 2015. In conclusion, the caries decline in 35-44-year old adults in Germany seems to be more pronounced than prior data would suggest, possibly due to aging of cohorts exposed to structured preventive programs in childhood (start 1989).

\section{6}

\section{Withdrawn}




\section{7}

\section{MIH has a Negative Impact on the Quality of Life of 8-10 Year-Old Children}

T.T. Guimarães , G.C.A. Americano ${ }^{\mathrm{a}, \mathrm{b}}$, J.A. Machado, G.S.A. Gonçalves ${ }^{\mathrm{a}}$, V.M. Soviero*a,b

verasoviero@gmail.com

aPediatric Dentistry Department, Universidade do Estado do Rio de Janeiro, Rio de Janeiro, Brazil; 'b School of Dentistry,

Faculdade Arthur Sá Earp Neto, Petrópolis, Brazil

Children with molar-incisor-hypomineralization (MIH) experience dental treatment significantly more frequent than children without MIH. Other usual clinical implications include dental fear, esthetic problems, and sensitive teeth. The aim of this study was to evaluate the impact of MIH on oral health related quality of life (QoL) in 8-10 year-old children. Sixty children with MIH, who was attending their first dental appointment at the clinic of the Pediatric Dentistry Department, were enrolled in the study. A comparative group without MIH was formed matching age and gender. Two calibrated examiners assessed $\mathrm{MIH}$ and dental caries using EAPD and WHO criteria, respectively. The child's perception questionnaire (CPQ8-10) was used to evaluate the impact of $\mathrm{MIH}$ on the QoL. CPQ8-10 consists of 29 questions divided among four domains: oral symptoms (OS), functional limitation (FL), emotional well-being (EWB), and social well-being (SWB). Non-parametric tests were used to test the association between CPQ and independent variables ( $\mathrm{MIH}$, affected incisors, molars severely affected, caries experience, treatment need, gender, and age). Poisson regression with robust variance was used for multivariate analysis to test the association between the CPQ8-10 domains and the independent variables in terms of rate ratio and $95 \%$ confidence interval (CI). Overall CPQ8-10, SO, and EWB scores were significantly higher for children with $\mathrm{MIH}(\mathrm{p}<0.05)$. The multivariate analysis showed that SO (RR: 1.37; 95\% CI: 1.02-1.84) and FL (RR: 1.56; $95 \%$ CI: $1.04-2.33$ ) were significantly affected by the presence of severely affected molars with treatment need. MIH had a negative impact on the QoL of children with MIH who showed higher values of CPQ8-10. The presence of severely affected molars with treatment need represented the main impact factor.

Funded by CAPES.

\section{8}

Factors Associated with Dental Caries Among a Population of Moroccan Adolescents: Principal Component Analysis

S. Chala ${ }^{\mathrm{a}-\mathrm{d}}$, F. Abdallaoui ${ }^{\mathrm{a}}$, A. Bour ${ }^{\mathrm{d}}$, R. Abouqal ${ }^{\mathrm{b}}$

Dr_chala@yahoo.fr

aDepartment of Endodontics and Restorative Dentistry, Faculty of Dentistry, Oral Biology and Biotechnology Laboraty, Mohammed V University in Rabat, Rabat, Morocco; bLaboratory of Biostatistics, Clinical and Epidemiological Research, Faculty of Medicine and Pharmacy, Mohammed V University in Rabat, Rabat, Morocco; ' ${ }^{\top}$ Mohammed V Military Teaching Hospital, Rabat, Morocco; ${ }^{\mathrm{d}}$ Research team on dietary and nutritional transition, University Ibn Tofail, Kenitra, Morocco

Factors associated with DMFT index has extensively been studied using regression analysis methods. Many of this studies used quantitatives variables as covariates, however, no study used data reduction tools like principal component analysis. The aim of the study was to summarize multidimensional data and provide continuous scores to be used for regression analysis. This cross-sectional study involves 540 adolescents recruited from secondary schools in Temara city, Morocco. Variables studied were oral health behavioral factors, including brushing habits, fluoridated toothpaste use, the overall frequency of sugar intake, smoking status, demographics and parents' educational level. Clinical variables included the DMFT according to WHO, Gingival index and Plaque Index. Principal component analysis using decayed teeth, missing teeth, filling teeth, Gingival index and Plaque Index for the extraction of factors that account for the maximum proportion of the variance in the data. Linear regression analysis was then performed to identify factors associated with generated sub-scores. Principal components analysis identified three components which explained $76 \%$ of the variance. These factors are labelled: a decayed factor, missing and filling factor, and gingival and plaque factor. Factors associated with decayed factor using multiple linear regression analysis were father primary education level $(p=0.01)$, mothers education level $(\mathrm{p}=0.03)$ and daily sugar consumption (0.05). In conclusion missing and filling teeth loaded onto a different factor from decayed teeth. These finding suggest that different components can be identified in populations, and may be used either to study associations with different aspects of oral health measures or help in the design of longitudinal studies of trajectories of dental caries risks.

Funded by Mohammed V university in Rabat, CNRST, Morocco. 
79

Oral Health and Socio-Demographic Characteristics at Geriatric Patients in Macedonia with Different Types of Edentulism

\author{
N. Stavreva*a , B. Kapusevska a , A. Sotirovska Ivkovska ${ }^{\text {, }}$ \\ A. Mijoska ${ }^{\mathrm{a}}$, E. Zabokova Bilbilova ${ }^{\mathrm{b}}$, V. Ambarkova ${ }^{\mathrm{b}}$ \\ natasha_stavreva@yahoo.com \\ aDepartment of Prosthodontics, Faculty of Dentistry, \\ University St. Cyril and Methodius, Skopje, Macedonia; \\ ${ }^{b}$ Department of Pediatric and Preventive Dentistry, Faculty \\ of Dentistry, University St. Cyril and Methodius, Skopje, \\ Macedonia
}

Health is the main component of quality of life, while the oral health is component of general health. The socio-demographic characteristics for epidemiological studies are important factors for perception of oral health and quality of life. Main purpose of this study was to perform an evaluation of the quality of life at geriatric patients (older than 65) with built-in oral prosthetic dentures with different type of edentulism (upper/lower total denture, upper/lower partial denture, upper partial and lower total denture, upper total and lower partial denture, only upper or only lower total denture, only upper or only lower partial denture) depending on the variables such as: affiliation, level of education and place of living, as socio-demographic characteristics. The survey was a prospective transversal epidemiological study conducted among 165 institutionally sheltered patients at Gerontology Institute (inspected group - IG) and 170 patients from the dental specialist clinics (control group CG) at age 65 and older. Geriatric Oral Health Assessment Index (GOHAI indicator) was used for obtaining the epidemiological data from the geriatric patients. Statistical program SPSS for Windows ver 13.0 was used for statistical processing. Chisquare test, $t$-test, Anova-Manova analysis were used for testing the significance of the differences between the variables, where the values for $\mathrm{p}<0.05$ were statistically significant. Patients with upper and lower total dentures dominated (43.6\% vs. $26.5 \%)$. The highest GOHAI score was observed in "other ethnics" of IC (32.08) and Romani-Gyspies of IG (31.00), while the lowest was in the Albanians of CG (25.91). Highest value of 30.15 had the IG of patients living in urban areas.

The quality of life and oral health of geriatric patients are at unsatisfactory level, with the major significant influence being place of residence while ehnicity and level of education were not significant factors.
80

Salivary Parameters Among COPD Patients

E. Dolenec ${ }^{\mathrm{a}}$, M. Mastnak ${ }^{\mathrm{b}}$, J. Krapežăb ${ }^{\mathrm{b}}$, M. Terčelj ${ }^{\mathrm{c}}$, B. Salobirc, J. Jan*a,b

janja.jan@mf.uni-lj.si

aDepartment of Dental Diseases and Endodontics, Medical Faculty, University of Ljubljana, Ljubljana, Slovenia;

bDepartment of Dental Diseases, University Medical Centre Ljubljana, Ljubljana, Slovenia; 'Department for Respiratory and Allergic Diseases, University Medical Centre Ljubljana, Ljubljana, Slovenia

Chronic obstructive pulmonary disease (COPD) is a highly prevalent illness. We have shown that COPD patients have higher caries prevalence. The aim of this pilot study was to evaluate salivary parameters among COPD patients. The study population consisted of 53-83-year old COPD patients $(n=50$, mean age 65.9) stage B-D according to GOLD guidelines. Fifty healthy controls were matched for age, sex, and smoking status. Measurements of dental caries, salivary flow, buffer capacity, colony densities of mutans streptococci and lactobacillus in stimulated saliva, and unstimulated salivary $\mathrm{pH}$ were conducted. The two groups were compared statistically using Chi-Square and Mann-Whitney-U tests. $\mathrm{D}_{12} \mathrm{MFT}$ index values were higher in COPD patients (COPD: $24.62 \pm 3.61$; controls: $21.35 \pm 4.35 ; \mathrm{p} \leq 0.01)$. Stimulated salivary flow rate was significantly $(\mathrm{p} \leq 0.05)$ lower in COPD patients $(1.07 \pm 0.65 \mathrm{~mL} / \mathrm{min})$ than in their matched controls $(1.62 \pm 0.66$ $\mathrm{mL} / \mathrm{min})$. Similarly, unstimulated salivary flow rate was lower (COPD: $0.28 \pm 0.18 \mathrm{~mL} / \mathrm{min}$; controls: $0.43 \pm 0.23 \mathrm{~mL} / \mathrm{min} ; \mathrm{p} \leq$ $0.05)$. In COPD patients higher colony densities of mutans streptococci (COPD: $2.72 \pm 0.83$; controls: $2.05 \pm 0.97$; $\leq$ 0.05) and of lactobacillus (COPD: $2.84 \pm 0.86$; controls: $2.00 \pm 0.94$; $\leq 50.01$ ) were found. Unstimulated salivary $\mathrm{pH}$ was lower in COPD patients (COPD: $6.93 \pm 0.61$; controls: $7.38 \pm 0.63$; $\mathrm{p} \leq$ 0.05). Buffer capacity of stimulated saliva samples was not significantly different (COPD: $1.67 \pm 0.51$; controls: $1.46 \pm 0.50$ ). The results showed that COPD patients had lower salivary flow, higher mutans streptococci and lactobacillus colony densities, and lower unstimulated salivary $\mathrm{pH}$.

Supported by Slovenian Ministry of Science and Education (P3-0374) and University Medical Centre Ljubljana (grant No. 20140257). 
81

\section{School Beverage Policies in Dutch-Speaking Belgium}

F. Marro*a, W. Jacquet ${ }^{\mathrm{b}, \mathrm{c}}$, P. Bottenberg ${ }^{\mathrm{b}}$, L. Martens ${ }^{\mathrm{a}}$

franmarrom@gmail.com

${ }^{a}$ Department of Paediatric Dentistry, PAECOMEDIS research

cluster, Gent University, Gent, Belgium; ' Oral Health Research Group ORHE, Faculty of Medicine and Pharmacy VUB Vrije

Universiteit Brussel, Brussels, Belgium; 'Department of

Educational Sciences EDWE-LOCl, Faculty of Psychology and

Educational Sciences, VUB Vrije Universiteit Brussel, Brussels, Belgium

Recently a prevalence study of Erosive tooth wear (ETW) in Belgium showed that type of education (vocational/technical) was a predictive variable for ETW risk in adolescents (OR: 1.49; 95\% CI: 1.03-2.13). Therefore, the aim of this study was to survey school policies related to the consumption of acidic beverages, and identify socio-demographic differences in such policies with respect to school type in Flanders, Belgium. All Flemish schools $(\mathrm{n}=$ 680 ) were asked to complete a validated questionnaire concerning school policies or regulations for acidic beverage consumption in their establishments. For data analysis, schools were grouped by type of education (General education: GSE or Vocational/technical education: VSE) and Chi-square t test was used to compare differences between groups. Thirty-four percent of the schools $(\mathrm{n}=$ 236) completed correctly the survey. Overall, $50 \%$ of the schools in Flanders reported to have written regulations concerning the offer and consumption of acidic beverages. Comparisons between type of educations indicated that significantly more GSE schools promoted the consumption of water in their curriculum $(p<0.05)$ and reported to limit the consumption of soft drinks and fruit juices to their pupils when compared with the VSE schools $(\mathrm{p}<0.05)$. In conclusion, school's policies concerning acidic beverage consumption varied significantly among education type in Flanders, which could influence the consumption habits of their pupils.

\section{2 \\ Relationship Between Caries Patterns in Early Childhood Caries in Bengaluru, India}

Latha Anandakrishna, S. Rachana, G. Shwetha

latha.pe.ds@msruas.ac.in

Department of Pedodontics and Preventive Dentistry, Faculty of Dental Sciences, MS Ramaiah University of Applied Sciences, Bengaluru, India

Early Childhood Caries(ECC) affecting toddlers and young children has become a major dental disease with prevalence rate of over $50 \%$ in India. Hence, it is imperative that any risk factor specific to ECC is addressed and mitigated. Children with ECC exhibit certain patterns of caries in both anterior and posterior region. These include the proximal, labial and occlusal surfaces of the primary dentition with differing etiological or risk factors. It could be hypothesized that specific caries pattern in the primary anterior could be associated with caries in posterior teeth.
The aim of the study was to assess the distribution of caries surface patterns in children with ECC and S-ECC and to check for association if any. Total of 1952 children aged between 24-71 months were screened to obtain the statistically determined sample of 375 with ECC and S-ECC. Oral Examination was conducted by a single examiner who was calibrated with kappa value of 1.0 for the criteria specified for WHO for dental caries for d component. Descriptive analysis of the data revealed that for children with ECC in the anterior region, maxillary anterior caries was more than caries in the mandibular anterior ( 279 vs 60 children). Anterior Proximal caries pattern was more prevalent than labial caries (252 vs 181 children). However, in the posterior region, mandibular teeth were more affected than maxillary (290 Vs 242 children). Pit and fissure caries was more prevalent than posterior proximal caries (307 vs 149 children). Association between anterior and posterior caries patterns was found to be statistically significant (chi square value $=20.057, \mathrm{df}=1, \mathrm{p}=0.000$ ). The research findings can be utilized to develop protocols to include stringent caries detection of both anterior and posterior regions for prevention strategy in ECC.

\section{3}

\section{Withdrawn}

\section{4}

\section{Application of CAST Index in Adult Population - Pilot} Study

J. Baginska a , E. Rodakowskab , I. Kaminskac, A. Masłowska a , M. Szpakowicz d , M. Łapińska ${ }^{\text {d }}$, Z. Stachurska d , J. Jamiolkowskid, A. Kierklo ${ }^{\mathrm{a}}$, K. Kaminskid

jbaginska@wp.pl

aDepartment of Dentistry Propaedeutics, Medical University

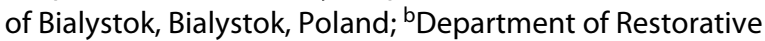
Dentistry, Medical University of Bialystok, Bialystok, Poland; 'Department of Integrated Dentistry, Medical University of Bialystok, Bialystok, Poland; 'Department of Population Medicine and Civilization Diseases Prevention, Medical University of Bialystok, Bialystok, Poland

The Caries Assessment and Spectrum Index (CAST) is intended for epidemiological analysis, but reports on its application in adult populations are lacking. The aim of this study was to evaluate the caries experience in adult population according to the CAST index. The pilot study was conducted as part of the project "Bialystok Polish Longitudinal University Study; Bialystok PLUS”. 134 randomly selected people were examined. They were divided into 3 age groups: $20-44$ years ( 46 people), $45-64$ years ( 42 people), and 65 and above ( 46 people). The mean numbers of teeth with particular CAST codes were calculated and analysed with the KruskalWallis test. The mean values of CAST according to the age group, respectively, were: CAST 0: $12.27 \pm 5.87,9 \pm 5.61$ and 5.09 \pm 4.73; CAST 1: $1.26 \pm 3.64$ in $20-44$ year-olds and 0 in other groups; CAST $2: 10.7 \pm 5.4,8.33 \pm 5.24$ and $5.13 \pm 5.43$; CAST $3: 0.78 \pm$ 
$2.25,0.57 \pm 1.35$ and $0.09 \pm 0.41$; CAST $4: 0.13 \pm 0.5,0.14 \pm 0.68$ and 0; CAST 5: $0.39 \pm 0.91,0.48 \pm 1.06$ and $0.26 \pm 0.8$; CAST 6 : $0.09 \pm 0.41,0.48 \pm 1.06$ and $0.09 \pm 0.41$; CAST 7: 0 ; CAST $8: 1.28 \pm$ $1.7,7.71 \pm 6.84$ and $14.98 \pm 9.18$; CAST 9: $0.8 \pm 2.65,1.24 \pm 2.54$ and $1.98 \pm 3.43$. There were statistically significant differences among the age groups in the number of healthy teeth (CAST 0), teeth with a fissure sealant (CAST 1), filled teeth (CAST 2), teeth with a pulp reaching cavity (CAST 6 ) and extracted teeth (CAST 8 ). The mean number of teeth with CAST $0,1,2$ decreased with age, CAST 6 was highest in the middle age group and the mean number of extracted teeth increased with age. Our results proved that CAST could be applied in adults. The CAST index helps to reveal age-related changes in the caries profile.

This study was supported by the Bialystok Municipal Office that financed the project - "Community Assessment Concerning the Risk of Lifestyle Diseases in the Population of Bialystok".

\section{5 \\ Fluoride Content Knowledge and Toothpaste Use Among Moscow 12 and 15-Year-Olds}

D. Said*, E. Kouzmina, P. Kuznetsov, I. Kuzmina

irinakuzmina.dent@mail.ru

Preventive Dentistry Department, Moscow State University of Medicine and Dentistry, Moscow, Russia

The aim of present study was to investigate the knowledge of fluoride content in toothpaste among the 12- and 15-yr-olds in Moscow. 735 of 12 and 15-year-olds were randomly selected to be involved in the study as part of 3rd National Oral Health Survey. Among them, 149 were interviewed regarding their knowledge of fluoride content in toothpaste. $47 \%$ of children used fluoridated toothpaste, $21 \%$ used non-fluoridated, $32 \%$ were unsure of F-content. Among those using F-toothpaste, only 5\% knew the F-concentration. For $16 \%$, the toothpaste was adviced by of dentists, $2 \%$ by hygienists, $59 \%$ by parents and $9 \%$ by advertising. The caries prevention effect of fluoride is known by $27 \%$. Toothpaste was recommended by dentists in $48 \%$ of cases, and among them $42 \%$ recommended that toothpaste should contain fluoride. However, only $14 \%$ of dentists advised a specific concentration of F. Among them, $13 \%$ recommended 500 ppm, 3\% 1450 ppm, 5\% 1600 ppm, and in $79 \%$ of cases there was no answer. Among the interviewed children, $14 \%$ based their choice of toothpaste on flavor, $29 \%$ on content, $6 \%$ on package design, $24 \%$ on recommendation, $10 \%$ on parents' choice, and $1 \%$ on price. In conclusion, despite the wide availability of fluoridated toothpaste, there is variance in F-concentration. Moreover, the patients' knowledge is insufficient, with a possible lack of clarity from the dentist based on patient feedback. Therefore attention should be paid to the correct F toothpaste usage while analyzing and planning oral health promotion programs among the 12 and 15-yr-olds in Moscow.

\section{6}

Association Between Obesity and Dental Caries Among Schoolchildren from South Brazil: A Longitudinal Study

\author{
N.C. Lock ${ }^{\mathrm{a}}$, C. Susin ${ }^{\mathrm{b}}$, C.D. Brusius ${ }^{\mathrm{c}}$, M. Maltz' , L. S. Alves*a \\ luanaseal@gmail.com \\ aDepartment of Restorative Dentistry, School of Dentistry, \\ Federal University of Santa Maria, Santa Maria, Brazil; \\ ${ }^{b}$ Department of Periodontics and Oral Biology, The Dental \\ College of Georgia, Augusta, USA; 'Department of Social \\ and Preventive Dentistry, Faculty of Odontology, Federal \\ University of Rio Grande do Sul, Porto Alegre, Brazil
}

The aim was to assess the association between weight status at 12 years and changes in DMFS among schoolchildren from South Brazil after a 2.5 -year period.

A total of 801 schoolchildren attending 42 public and private schools were examined at baseline (12 years of age) and after 2.5 years $(S D=0.3)$. Data collection included a structured questionnaire on socio-demographic information and oral hygiene habits, the recording of anthropometric measures (height and weight) and caries examination (non-cavitated and cavitated, inactive and active caries lesions). Two examiners performed caries examination, one at baseline (intra-examiner kappa value $\geq 0.80$ ) and another at follow-up (intra-examiner kappa value $\geq 0.81$ ), one calibrated by the other (inter-examiner kappa value $=0.78$ ). The primary outcome was change in DMFS, defined as the difference between follow-up and baseline DMFS. Weight status based on BMI (Body Mass Index)-for-age Z-score was considered the main predictor variable. Preliminary analysis used the Wald test. The association between predictor variables and DMFS changes used negative binomial regression models (unadjusted and adjusted). Rate ratios and their $95 \%$ confidence intervals were estimated. A multivariable fractional polynomial model was used to further explore the relationship between obesity and dental caries. Baseline DMFS (+increment) were $2.80(+0.86), 2.40(+0.91)$, and 2.43 $(+0.42)$ for normal weight, overweight, and obese schoolchildren, respectively. Obese adolescents had significantly lower DMFS changes than normal weight ones $(0.42$ versus 0.86 , respectively, $\mathrm{p}<0.05)$. In the risk assessment analysis, no association between weight status and DMFS changes was observed (overweight, adjusted $\mathrm{IRR}=0.98,95 \% \mathrm{CI}=0.73-1.31$; obese, adjusted $\mathrm{IRR}=0.77$, $95 \% \mathrm{CI}=0.51-1.16)$. However, the multivariable fractional polynomial model showed an inverted $U$ shaped relationship with a decreasing DMFS with increasing BMI $(\mathrm{p}<0.05)$. In conclusion, this population-based longitudinal study showed that obese adolescents showed lower DMFS changes over a 2.5-year follow-up period than normal weight individuals.

Funded by CAPES and PROPESQ/UFRGS. 


\section{7}

\section{Progression of Non-Cavitated Caries Lesions According to Lesion Activity: A 2.5-Year Longitudinal Study}

\author{
F.L. Leal', L.S. Alves a , M.B. Wagner ${ }^{\text {b }}$, J.E.A. Zenkner*a, C. Susin', \\ C.D. Brusius ${ }^{\mathrm{d}}$, M. Maltz ${ }^{\mathrm{d}}$ \\ jezenkner@gmail.com \\ ${ }^{\text {aS }}$ Chool of Dentistry, Federal University of Santa Maria, Santa \\ Maria, Brazil; b Faculty of Medicine, Federal University of Rio \\ Grande do Sul, Porto Alegre, Brazil; 'The Dental College of \\ Georgia, Augusta, USA; dFaculty of Odontology, Federal \\ University of Rio Grande do Sul, Porto Alegre, Brazil
}

The aim of this longitudinal study was to evaluate the pattern of progression of inactive non-cavitated (INC) and active non-cavitated (ANC) caries lesions in a population-based sample of South Brazilian adolescents in order to validate the visual caries activity assessment. A total of 801 schoolchildren were examined at baseline ( 12 years) and after a mean time interval of 2.5 years. Data collection included the application of questionnaire and clinical examination. The primary outcome of this study was caries progression, defined as the presence of cavity, dark shadow from dentine, filling or extraction at the follow-up examination. Negative binomial regression models (unadjusted and adjusted) were used to estimate the risk for caries progression over the study period. Models were fitted using generalized estimating equations following a hierarchical approach. The main predictor variable was the status of the surface at baseline: sound, INC lesion, or ANC lesion. At baseline, a single examiner obtained an overall unweighted Cohen's kappa value of 0.84 for caries examination (per category, it was $0.90,0.75$, and 0.81 for sound surfaces, INC, and ANC lesions, respectively). A total of 19,438 permanent teeth and 46,238 tooth surfaces were included. After 2.5 years, progression rates of $1.0 \%$, $9.0 \%$, and $12.6 \%$ were found for sound surfaces, INC lesions, and ANC lesions, respectively. In the adjusted model, it was observed that INC and ANC lesions had an approximately 5-fold increased risk for caries progression than did sound surfaces (IRR $=5.35$, 95\% CI $=4.20-6.80$ and $\mathrm{IRR}=4.97,95 \% \mathrm{CI}=3.44-7.19$, respectively). In conclusion, this longitudinal study found a similar pattern of progression for inactive and active non-cavitated caries lesions among South Brazilian adolescents. Our findings emphasize the difficulty of classifying caries activity in low caries prevalence populations.

Funded by CAPES and PROPESQ/UFRGS.

\section{8 \\ Objective and Subjective Evaluation of Oral Health in Coronary Patients from Euroaspire Project}

E. Rodakowska*a, J. Baginska ${ }^{\text {b }}$, I. Kaminskac, J. Jamiolkowski ${ }^{\text {, }}$, M. Lapinska ${ }^{d}$, M. Szpakowicz d , Z. Stachurska ${ }^{\text {d }}$, K. Kaminski $^{\mathrm{d}}$

ewarodakowska@interia.pl

aDepartment of Restorative Dentistry, Medical University of Bialystok, Bialystok, Poland; ${ }^{b}$ Department of Dentistry Propaedeutics, Medical University of Bialystok, Bialystok, Poland; 'Department of Integrated Dentistry, Medical University of Bialystok, Bialystok, Poland; dDepartment of Population Medicine and Civilization Diseases Prevention, Medical University of Bialystok, Bialystok, Poland

The relationship between oral health and general health is particularly important in the group of patients with cardiovascular diseases. The aim of the study was to evaluate association between objective (dentist) and subjective (OHIP-14 scale) assessment of oral health in coronary patients from Euroaspire project. This was cross-sectional study. Objective evaluation included oral examination provided by dentist: number of teeth $(\leq 20 ;>20)$, dental status (dentate/edentate), decay, filled teeth, API index. Subjective evaluation included Polish version of Oral Health Impact Profile-14. Other variables included age $(<65 ; \geq 65)$, education, dry mouth, chewing problems. The final sample consisted of 146 patients (mean age $65.17 ; 73.2 \%$ men). The mean number of teeth was 12.75. Majority of patients had secondary education, had less than 20 teeth, $83 \%$ had API over $70 \%$, declared chewing problems and dry mouth, mean number of decay teeth was 1.07 , filled teeth was 3.62; mean OHIP-14 was 20.29. Only chewing problems ( $\mathrm{p}=$ $0.000)$ and dry mouth $(\mathrm{p}=0.015)$ were significantly associated with mean OHIP-14. Conclusions: The condition of oral health affects the course and prognosis of cardiovascular diseases. Besides, risk factors of myocardial infraction include poor oral hygiene and tooth loss. The objective oral evaluation demonstrated need of improvement of oral health. The subjective evaluation provided by patients did not fully reflect their oral health problems.

Funded by grant from Medical University of Białystok, Poland.

89

Caries Experience Assessed by ICDAS and DMFS Index in 6-, 12-, and 15-Year-Olds in Moscow

I. Kuzmina*a, D. Said ${ }^{\mathrm{a}}$, K.R. Ekstrand ${ }^{\mathrm{b}}$

irinakuzmina.dent@mail.ru

aDepartment of Preventive Dentistry, Moscow State University of Medicine and Dentistry, Moscow, Russia; ${ }^{b}$ Research area

for Cariology and Endodontics, Department of Odontology,

Faculty of Health and Medicine, University of Copenhagen,

Copenhagen, Denmark

This study aimed to evaluate the caries experience using ICDAS and the DMFS index in 6-, 12- and 15-year-olds in Moscow. As a part of the 3rd National Oral Health Survey, 1229 children aged 6-, 12- and 15-years old were randomly selected in Moscow. 
Among them, 216 children were examined using both the ICDAS (D1-D6) and the D3MFS index. Examinations were done by two dentists, previously trained by an experienced examiner. Reexaminations of 20 patients were done, where the second examinations were performed three days after the first ones. The reproducibilities (inter/intra) of the ICDAS-scores assessed by Kappa were $\geq 0.70$. Similar data for the traditional DMFS were $\geq 0.85$. Combining D1-D2 scores the means were 1.37 (1.53), 4.27 (5.50), 7.44 (7.34), respectively. Combining D3-D4 scores the means were 0.37 (0.87), 0.43 (1.10), 1.29 (2.35), respectively. Finally, combining D5D6 scores the mean was $0.14(0.77)$ and was observed only in 15-years-olds. The MFS scores were 0.48 (1.96), 1.65 (2.41), and 2.76 (3.70) in the three age groups, respectively. More than $70 \%$ of the 15-yr-olds had one or more surfaces with ICDAS scores or surfaces which were filled or extracted due to caries. The conventional mean D3MFS for corresponding age were 0.85 (2.16), 2.08 (2.52) and 4.24 (4.72), respectively. Thus, the ICDAS system increased the mean caries level by about 3 times for all three age groups examined. The data indicate that the caries experience is moderately high among children in Moscow. The use of ICDAS gives us much more information about the caries status compared to the traditional DMFS index. The extra information is of paramount interest for better planning of prevention strategies among the young population in Russia.

\section{0 \\ The Use of Caries Risk Assessment Among Dutch Dentists \\ M.H. van der Veen ${ }^{\mathrm{a}, \mathrm{b}}$, J.J. de Soet ${ }^{\mathrm{a}}$, S. Döpp ${ }^{\mathrm{b}}$, C.M.C. Volgenant*a \\ m.vd.veen@acta.nl \\ apreventive Dentistry, ACTA, Amsterdam, The Netherlands; \\ bOral Hygiene, Inholland University of Applied Sciences, \\ Amsterdam, The Netherlands}

The aim of this study was to determine the percentage of the Dutch dentists using caries risk assessment (CRA) and the relevance they give to specific risk factors.

A questionnaire [Doméjean et al.: Clin Oral Invest, 2016] was distributed among a random sample of 1500 Dutch dentists. Questions were asked regarding demographics, use of CRA and relevance of risk factors. The relevance of specific risk factors was ranked on a 5 point Likert scale and as top 3 for permanent and primary/mixed dentition. Three hundred and fifteen dentists responded (52\% male), of which $78.8 \%$ apply CRA. $11.8 \%$ use a standard form. Women apply CRA more often than men (Women $83.8 \%$, Men $73.2 \%$; $\left.\chi^{2}=4.489, \mathrm{p}=0.034\right)$. CRA is more often applied by dentists working in group practices compared to solo practices (Group 84.7\%, Solo 73.8\%; $\chi^{2}=4.742, \mathrm{p}=0.029$ ). The mean age of dentists applying CRA $(44.5 \mathrm{yr} \pm 12.1 \mathrm{yr})$ is lower than that of those who do not $(49.1 \mathrm{yr} \pm 12.9 \mathrm{yr}, \mathrm{U}=4748, \mathrm{p}=0.013)$. The five risk factors given most often as part of the top 3 for permanent dentition were oral hygiene (246), diet (161), presence of large restorations (132), recent caries development (120) and patient's knowledge about caries (46) and for primary/mixed dentition: oral hygiene (200), diet (142), patient's/parental knowledge about caries (138) presence active caries lesion (100) and recent caries development (70). This did not match the ranking of individual risk factors on a Likert-scale, indicating a lack of knowledge among dentists. In conclusion, the majority of Dutch dentists in the study sample use CRA. The factors included in CRA may differ among dentists. Knowledge on relevance of risk factors and their contribution to caries risk should be improved and may be aided by increasing the use of a standard form.

\section{1}

\section{Colombian Clinicians Implementation of ICCMS ${ }^{\text {TM }}$ Recommended Caries Diagnosis and Management Behaviours}

N. Abreu Placeres*a,b, J.T. Newton ${ }^{c}$, N. Pitts ${ }^{\mathrm{d}}$, K.R. Ekstrand ${ }^{\mathrm{e}}$, V. Ávila ${ }^{\mathrm{a}}$, S. Martignon ${ }^{\mathrm{a}, \mathrm{d}}$

n.abreu@prof.unibe.edu.do

aUNICA - Caries Research Unit, Research Vice-rectory, Universidad El Bosque, Bogotá, Colombia; ${ }^{b}$ Biomaterials and Dentistry Research Center (CIBO-UNIBE), Academic Research Department, Universidad Iberoamericana, Santo Domingo, Dominican Republic; 'Social \& Behavioural Sciences, Kings College London Dental Institute, London, United Kingdom; ${ }^{\mathrm{d} D e n t a l}$ Innovation and Translation Centre, Kings College London Dental Institute, London, United Kingdom; 'Section of Cariology and Endodontics, School of Dentistry, Faculty of Health and Medical Sciences, University of Copenhagen, Copenhagen, Denmark

The Alliance for a Cavity Free Future-Colombian Chapter (ACFF-CC) has been working assiduously in the adoption of best caries management practices. Proof of this is the implementation of the Cariology Consensus in Colombia's dental schools. The International Caries Classification and Management System (ICC$\mathrm{MS}^{\mathrm{TM}}$ ) intends to deliver best-evidence caries management recommendations for clinicians to offer patients better dental care; the ACFF-CC promotes ICCMS ${ }^{\mathrm{TM}}$. The aim was to assess Colombian clinicians implementation of ICCMS ${ }^{\mathrm{TM}}$ recommended caries diagnosis and management behaviours, and to determine which COM$B$ model components better explain these behaviours. Experimental approach: A previously validated 79-item questionnaire that measured Capability, Opportunity, and Motivation to conduct caries diagnosis and management Behaviours (COM-B model), was answered by 489 randomly-selected clinicians from the eight largest Colombian cities. The Capability, Opportunity, and Behaviour items were answered via a 1-5 scale, while a $0-5$ scale was used for the Motivation items. The most-often performed diagnosis behaviour was initial/moderate caries-lesion assessment (4.17 \pm $0.99)$, while bitewing-radiograph was the least $(3.22 \pm 1.04)$. In both cases Opportunity (Resources) was the best predictor of these behaviours $(\beta=0.364$ and $0.461 ; p<0.001)$. For caries management, tooth-preserving-operative care $(4.74 \pm 0.57)$ and restoration reparation $(4.32 \pm 0.89)$ were the most frequently conducted. Here, Capability was the best predictor $(\beta=0.420$ and 0.317 ; $<$ $0.001)$. Conversely, sealants as treatment for initial-caries lesions $(2.66 \pm 1.21)$ and moderate-caries lesions non-operative treatment $(2.94 \pm 1.34)$ were the least implemented. For both, Opportunity 
(Relevance) was the best predictor $(\beta=0.335$ and $0.414 ; \mathrm{p}<0.001)$. In conclusions, in general clinicians report that they are conducting ICCMS ${ }^{\mathrm{TM}}$ best practices for caries diagnosis and management 'most of the time', with room for improvement on some recommended behaviours. An improvement in the Capability and Opportunity components may be needed to increase the implementation of some ICCMSTM recommended behaviours.

Funded by the Alliance for a Cavity Free Future, Universidad El Bosque, King's College London and Universidad Iberoamericana.

\section{2 \\ Calibration Outcomes of Paediatric Dentists and Master Students on the Merged-ICDAS Visual Caries Criteria}

R.M. Santamaria*a, C.H. Splieth ${ }^{\mathrm{a}}$, N. Pitts ${ }^{\mathrm{b}}$, M. Alkilzy ${ }^{\mathrm{a}}$, J. Schmoeckel', A. Al-Ani ${ }^{\text {a }}$, M.S. Mourad ${ }^{\mathrm{a}}$, S. Martignon ${ }^{\mathrm{b}, \mathrm{c}}$

ruth.santamaria@uni-greifswald.de

${ }^{a}$ Department of Preventive and Pediatric Dentistry, University

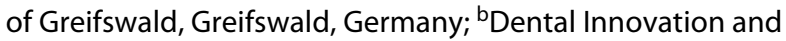
Translation Centre, King's College London Dental Institute, London, UK; ' CUNICA Caries Research Unit, Research Vicerectory, Universidad El Bosque, Bogotá, Colombia

The ICDAS visual caries criteria provide a standardised method for comprehensive caries classification proposed for adoption into clinical practice. ICDAS evolved into The International Caries Classification and Management System (ICC$\mathrm{MS}^{\mathrm{TM}}$ ) to provide a patient-centred caries management system. Recently, ICCMS ${ }^{\mathrm{TM}}$ developed an e-learning tool to support the training and calibration of examiners. To examine the effect of a two-day calibration on the reliability of coronal caries assessment on natural teeth using the merged-ICDAS criteria (initial [1-2], moderate [3-4] and extensive [5-6]) by a group of paediatric dentists (PDs) and PD master students (PDMS) not familiar with the system. Three experienced PDs and 10 PDMS attending a 3-year programme participated in the training. One week before training, participants had access to the e-learning system introduction programme. First-training day included: caries and ICDAS lectures and a pre-clinical workshop. Secondtraining day: pre-clinical/clinical workshops with time-structured examinations of natural teeth. Participants assessed 150 coronal surfaces of 31 primary/permanent teeth. To assess the intra-examiner reliability, 100 surfaces were re-examined in a different random order. Inter-examiner weighted Kappa rank values were $0.51-0.85$ for PDMS and $0.64-0.72$ for PDs. All PDs and most PDMS (60\%) reached a substantial agreement $(\mathrm{K}=$ 0.61-0.80), two PDMS (20\%) an almost perfect agreement $(\mathrm{K} \geq$ $0.81)$, while two reached only a moderate agreement $(\mathrm{K}=0.41-$ $0.60)$. Intra-examiner reliability ranks were: PDMS: $0.60-0.72$ and PDs: $0.60-0.67$. A substantial agreement $(K=0.61-0.80)$ was reached by $90 \%$ and $66 \%$, respectively. The level of experience (PDs vs. PDMS or study year) did not influence the calibration outcomes. These findings suggest that with the e-learning system introduction programme and a two-day calibration on assessment of coronal caries lesions using the ICDAS system it is possible to reach a substantial level of inter- and intra-examiner reliability.

Funded by the Department of Preventive and Pediatric Dentistry, University of Greifswald/Germany, Greifswald \& Global Collaboratory for Caries Management, ICCMS(TM), Dental Innovation and Translation Centre, King's College London Dental Institute, London, UK.

\section{3}

\section{Dental Health of Elderly People in Nursing Homes in Bogotá}

M.V. Usuga-Vacca*a, D.J. Marín ${ }^{\mathrm{b}}$, L.F. Gamboa ${ }^{\mathrm{a}}$, S. Martignon ${ }^{\mathrm{a}, \mathrm{c}}$

usugamargarita@unbosque.edu.co

aUNICA - Caries Research Unit, Research Vice-rectory, Universidad El Bosque, Bogotá, Colombia; 'bniversidad Nacional de Colombia, Sede Bogotá, Dental School, Colombia; 'Dental Innovation and Translation Centre, Kings College London, London, United Kingdom

The study aimed to describe the dental status of institutionalized elderly in Bogotá.

The study counted with Ethics approval and informed consent forms. A sample size of 180 subjects was calculated. Nursing-homes registered in the Bogota's Secretary of Health were invited to participate. Aged people ( $\geq 60$ years old) without severe mental disability or mouth-opening limitation were clinically assessed by three trained examiners in the nursing homes without tooth surfaces airdrying (epi), in terms of: -total/partial tooth loss, use of dental prosthesis; -Root caries lesions with ICDAS-epi modified system (R-ICDAS: Sound; Initial (depth $<0.5 \mathrm{~mm}$ ); Moderate-Extense (depth $\geq 0.5 \mathrm{~mm}$ ); -Coronal caries lesions with ICDAS-epi system (C-ICDAS-epi: Sound, Initial-epi; Moderate-Extense); Fillings (F) and Missing (M) surfaces/teeth, to account for: 1 . Coronal Conventional caries experience (Moderate-Extense caries lesions (D) plus $M$ and F: DMF): C-Conventional-DMF; 2. Coronal ICDAS caries experience (including Initial-epi caries lesions): C-ICDAS-epi-DMF; 3. Root Conventional caries experience (Root-caries-Index): RCI, and 4. Root ICDAS-epi caries experience (R-DF): R-ICDAS-epiDF. Of 29 institutions contacted, 23 were visited with 293 residents approached, of which 58 were excluded, for a sample size of $\mathrm{n}=235$. The subjects' mean age was of $82.8 \pm 8.8$ years $(69.8 \%$ female). A total of 133 participants $(56.5 \%)$ had at least one remaining tooth, with a mean of $6.8 \pm 8.2$ teeth. The prevalence of C-ConventionalDMF was of $100 \%$ with a mean number of teeth (T) C-Conventional-DMFT of $24.7 \pm 4.4$ and a mean number of surfaces (S) C-Conventional-DMFS of $108.5 \pm 25.6$. When including Initial-epi lesions (C-ICDAS-epi-DMFS) the later only increased to $108.7 \pm 25$. The prevalence of RCI was of $30.6 \%$ with a mean RCIT of $0.04 \pm 0.11$ and RCIS $0.6 \pm 1.5$. When including Initial lesions (R-ICDAS-epi-DF) prevalence increased to $31.5 \%$ and mean of surfaces (R-ICDAS-epiDFS) to $1.1 \pm 2.1$. Of the whole sample $43.4 \%(n=102)$ presented total tooth loss, of which $58.4 \%$ used total-denture prosthesis. Of those with no/partial tooth loss $(n=133), 32.3 \%$ used partial-removable bi/uni-maxillary prosthesis. Most prosthesis were defective (97.5\%). Dental health conditions in Bogotá nursing-home elders were unfavorable and don't meet the 2000 -WHO goal. 
This study was funded by Colciencias grant number 721-2016; Universidad El Bosque, Colombia; and Universidad Nacional de Colombia, Colombia.

\section{4}

\section{Withdrawn}

\section{5}

Association between Dental Health, Type of School, Socioeconomic- and Nutritional Status in 10-18 Year-Old Adolescents

F. Krause*a, J. Schmidta , M. Kreuz ${ }^{\mathrm{b}}$, C. Hirsch ${ }^{\mathrm{c}}$, M. Löffler ${ }^{\mathrm{b}, \mathrm{d}}$, W. Kiess ${ }^{\mathrm{d}-\mathrm{f}}$, K.-J. Park ${ }^{\mathrm{a}}$, D. Ziebolz ${ }^{\mathrm{a}}$, R. Haak ${ }^{\mathrm{a}}$

felix.krause@medizin.uni-leipzig.de

aDepartment of Cariology, Endodontology and

Periodontology, University of Leipzig, Leipzig, Germany;

b Institute for Medical Informatics, Statistics, and Epidemiology, Universität Leipzig, Leipzig, Germany; ' $D$ epartment of Pediatric and Preventive Dentistry, University of Leipzig, Leipzig, Germany; ${ }^{\mathrm{d}}$ Research Center for Civilization Diseases, University of Leipzig, Leipzig, Germany; ${ }^{\text {eDepartment }}$ of Women and Child Health, Hospital for Children and Adolescents and Center for Pediatric Research (CPL), University of Leipzig, Leipzig, Germany; Integrated Research and Treatment Center Adiposity Diseases, University of Leipzig, Leipzig, Germany

The aim was to assess caries experience among 10-to-18-yearolds, including initial lesions and dentine caries associated with school type, socioeconomic and nutritional status. Within the ep- idemiologic LIFE Child study, 1116 adolescents (10 to 18 years) were included in the present investigation. Dental caries (ICDASII criteria) and fillings were recorded on surface basis of six index teeth $(16,11,26,36,31,46)$. Additionally, univariate analyses were performed to determine the associations between caries experience and school type, socioeconomic status (SES) and nutritional status. Information about SES and school type was obtained by questionnaire survey. SES was grouped into "high", "middle" and "low". Nutritional status was recorded by the body-mass-indexstandard-deviation-score (BMI-SDS) using $1.5 \mathrm{SD}$ as the cut-off point. The associations between variables were analysed using Mann-Whitney-U and Kruskal-Wallis-test. Mean DF-S values $\left(\mathrm{D}_{5 \& 6} \mathrm{~F}-\mathrm{S}\right.$ and $\left.\mathrm{D}_{1-6} \mathrm{~F}-\mathrm{S}\right)$ among adolescents were age-related: 10 -to12 -year-olds had scores of 0.33 and 3.56 surfaces, whereas in the groups of $13-15$ and $16-18$ years $0.64 / 4.67$ and $1.00 / 5.65$ were found respectively $(\mathrm{p}<0.001)$. Type of school (grammar school vs. middle school) significantly influenced mean $\mathrm{D}_{5 \& 6} \mathrm{~F}-\mathrm{S}(0.5$ vs. 0.79, $\mathrm{p}<0.001$ ) and a trend was observed for $\mathrm{D}_{1-6} \mathrm{~F}-\mathrm{S}$ (4.3 vs. 5.0, $\mathrm{p}=$ $0.063)$. Regarding low vs. high SES, $\mathrm{D}_{5 \& 6} \mathrm{~F}-\mathrm{S}(0.98$ and $0.35, \mathrm{p}<$ $0.001)$ and $\mathrm{D}_{1-6} \mathrm{~F}-\mathrm{S}(5.74$ and $3.68, \mathrm{p}=0.001)$ were significantly different. Additionally, nutritional status significantly influenced mean values of $\mathrm{D}_{5 \& 6} \mathrm{~F}-\mathrm{S}(0.44$ (BMI-SDS $\leq 1.5)$ vs. 0.86 (BMI-SDS $>1.5 ; \mathrm{p}<0.001)$ and of $\mathrm{D}_{1-6} \mathrm{~F}-\mathrm{S}(4.02$ vs. $5.02 ; \mathrm{p}=0.001)$. Conclusions: There is association between dental caries experience and type of school, socioeconomic- and nutritional status among adolescents aged 10 to 18 years.

The study is supported by LIFE - Leipzig Research Center for Civilization Diseases, University of Leipzig. This project was funded by means of the European Social Fund and the Free State of Saxony. 


\section{Session 9 Fluoride Microbiology}

\section{6 \\ A Comparative Evaluation of Ion Release Characteristics of Three Different Fluoride Containing Dental Varnishes}

A. Sleibi*, A.R. Tappuni, N. Karpukhina, R. Hill, A. Baysan

dr_ahmedsm75@yahoo.com

Barts and The London School of Medicine and Dentistry, Institute of Dentistry, QMUL, London, UK

To assess the cumulative fluoride, calcium and phosphorus ion release characteristics from three dental fluoride varnishes either with CPP-ACP or Bioglass or fluoride alone using X-ray diffraction (XRD), Fourier-transform infrared spectroscopy (FTIR), and Nuclear magnetic resonance spectroscopy (NMR).

Three fluoride varnishes were selected; MI Varnish (GC, CPP$\mathrm{ACP}+5 \% \mathrm{NaF}$ ), Experimental (Dentsply Sirona, Bioglass $+5 \% \mathrm{NaF}$ ), and NUPRO White (Dentsply Sirona, $5 \% \mathrm{NaF}$ ). A thin layer of each varnish $(0.0674 \pm 0.0005 \mathrm{~g})$ was spread on an area of $20 \times 25 \mathrm{~mm}$ of a rough glass $(n=7)$. These were separately immersed in $10 \mathrm{ml}$ Tris buffer $(0.06 \mathrm{M}, \mathrm{pH}=7.30)$ and kept in a shaking incubator at $37^{\circ} \mathrm{C}$. The immersion solution was changed after 1, 2, 4, 6, 24 and $48 \mathrm{hrs}$. $\mathrm{F}$ ion concentration was measured at each time using an Ion Selective Electrode F-ISE (Orion 9609BNWP, Thermo Fisher, USA). Calcium and phosphate ion release was determined using Optical Emission Spectroscopy (Vista-PRO, Oxford, UK). XRD, FTIR and NMR analysis were performed before and following immersion. The cumulative F ion release was significantly higher in CPP-ACP $(1.113 \mathrm{mmol} / \mathrm{g})>$ bioglass $(0.638)>$ fluoride alone $(0.112)(\mathrm{p}<$ $0.001)$. The cumulative calcium and phosphorous ion were higher in the CPP-ACP $(0.137 \mathrm{mmol} / \mathrm{g}, 0.119)$ than bioglass $(0.067,0.015)$ respectively. The XRD and ${ }^{19} \mathrm{~F}$ MAS-NMR confirmed the presence of $\mathrm{NaF}$ peaks in all cases before immersion that turn into less prominent signal with the appearance of hydroxyapatite crystals after immersion. ${ }^{19} \mathrm{~F}$ MAS-NMR revealed $\mathrm{CaF}_{2}$ formation after immersion in both CPP-ACP and bioglass. ${ }^{31} \mathrm{P}$ MAS-NMR showed phosphate signal in both the CPP-ACP and bioglass before immersion. FTIR failed to show any sign of apatite formation. Either CPP-ACP or bioglass enhanced without compromising the bio- availability of fluoride ion release. CPP-ACP varnish was the most promising for fluoride, calcium and phosphate ion release.

This study was financially supported by the Ministry of Higher Education in Iraq as part of a PhD project. The dental companies GC (Japan) and Dentsply Sirona (USA) are appreciatively acknowledged for providing of the materials for this study.

\section{7 \\ Total, Soluble and Ionic Fluoride in Toothpastes from Uzbekistan and Belgium \\ P. Bottenberg*a,b, C. Vercruysse ${ }^{\mathrm{b}}$, A.G. Schulte ${ }^{\mathrm{c}}$, B. Khudanov ${ }^{\mathrm{d}}$ \\ pbottenb@vub.be \\ ${ }^{a}$ Oral Health Research Group, VUB, Brussels, Belgium; ${ }^{\text {bTissue }}$ Engineering and Biomaterials, University of Ghent, Ghent, Belgium; 'Department of Special Care Dentistry, Witten/ Herdecke University, Witten, Germany; ${ }^{\mathrm{d}}$ Tashkent State Dental Institute, Tashkent, Uzbekistan}

Many toothpastes in non-market-economy countries have low total or ionic fluoride content. The aim was to determine fluoride in various forms in toothpastes available in Uzbekistan. 12 different toothpaste samples were acquired in Tashkent (Uzbekistan), 3 from domestic production and 9 were import products from PR of China (5), Russian Federation (3) and EU (1). Two toothpastes were acquired in Belgium, one with Na-monofluorophosphate (MFP) and one with NaF which served as control. Fluoride was assayed according to the method of Cury et al. [Braz. Dent. J 2010; 21:369-400]. Toothpaste slurry was hydrolyzed with $2 \mathrm{~N} \mathrm{HCl}$ (total fluoride, TF) as well as its supernatant (Total Soluble Fluoride, TSF). Supernatant without acid hydrolysis was regarded as Ionizable Fluoride (IF). Fluoride and ionic calcium were determined potentiometrically. Of the test toothpastes only 6 had TF and TSF values above 1000 ppm (median TF: 1033, IQR: 814-1370; median TSF: 922, IQR: 423-1265). One toothpaste from domestic production had a TF of $740 \mathrm{ppm}$, two below the detection limit. Ionizable fluoride (median: 229, IQR: 229-926) was significantly lower than TF $(\mathrm{p}<0.001)$ or TSF $(\mathrm{p}<0.05$, Dunn's test). Only one of the tooth- 
pastes sold in Uzbekistan (EU produced) showed an IF value of $>80 \%$ of TF. The Belgian MFP toothpaste had low IF (280 ppm) although TF was in agreement with declaration, whereas the $\mathrm{NaF}$ toothpaste had high IF. None of the toothpastes contained ionizable calcium. Many toothpaste packages did not mention fluoride concentration or compound. If the compound was mentioned, it was mostly MFP. Most toothpastes acquired in Uzbekistan showed low ionizable fluoride. Some domestic products even failed to contain measurable quantities of fluoride. Quality control and labelling are issues that deserve the attention of healthcare professionals, industry and authorities, particularly in non-market-economy countries like Uzbekistan.

\section{8 \\ Fluoride Concentration and Reactivity with Carious Dentine of Silver Diamine Fluoride Products}

A.L. Soares-Yoshikawa*, J.A. Cury, C.P.M. Tabchoury

alaignier@yahoo.com.br

Piracicaba Dental School, University of Campinas, São Paulo, Brazil

The aim of this in vitro study was to determine the fluoride concentration in silver diamine fluoride (SDF) products and to evaluate their reactivity with carious dentin. The SDF products were: I. Advantage Arrest (45,283-51,013 ppm F); II: Ancárie 12\% (14,100 ppm F); III: Ancárie 30\% (35,400 ppm F), IV: Cariestop $12 \%$ (14,100 ppm F); V: Cariestop 30\% (35,400 ppm F) and VI: Saforide $38 \%$ (45,283 ppm F). Fluoride concentration in the products was evaluated with ISE (ion-specific electrode) by direct technique and after microdiffusion. For reactivity, dentin slabs (7 x 4 $\mathrm{mm}$ ) with caries-like lesions were sectioned in two halves. One half was used as control (treated with purified water) and the other for treatment with the products $(\mathrm{n}=10$ blocks/group; applied for 1 min). Loosely-bound (" $\mathrm{CaF}_{2}$ ") and firmly-bound fluoride (FA) were determined in both half slabs. The results found in the treated half slab was subtracted from the control and expressed in $\mu \mathrm{g} \mathrm{F}$ / $\mathrm{cm}^{2}$. Data were analyzed by one-way ANOVA and Tukey test. The fluoride concentration in the products (mean $\pm \mathrm{SD} ; \mathrm{ppm} \mathrm{F}$; w $/ \mathrm{v}$ ) by the ISE direct technique was: I. 57,249.4 $\pm 1,851.1$; II. 4,814.2 \pm 267.6; III. 5,726.2 \pm 42.9 ; IV. 10,144.5 \pm 467.5 ; V. 11,858.4 \pm 575.4 ; VI. $53,490.9 \pm 554$. These values were confirmed using the microdiffusion technique. The concentration (mean $\pm \mathrm{SD}$ ) of " $\mathrm{CaF}_{2}$ " and FA formed were, respectively: I. $89.9 \pm 34.1 \mathrm{~A}$; II. $72.8 \pm 26.2 \mathrm{AB}$; III. $98.0 \pm 28.5 \mathrm{~A}$; IV. $40.6 \pm 17.2 \mathrm{~B}$; V. $82.9 \pm 23.2 \mathrm{~A}$; VI. $75.9 \pm$ 25.4A; and I. $1.8 \pm 1.0 \mathrm{AB}$; II. $2.1 \pm 1.3 \mathrm{AB}$; III. $3.0 \pm 1.1 \mathrm{~B}$; IV. $1.6 \pm$ $1.2 \mathrm{~A}$; V. $1.2 \pm 0.6 \mathrm{~A}$; VI. $2.1 \pm 1.1 \mathrm{AB}$. The results showed that the reactivity of products analyzed with carious dentin was low and was not a function of fluoride concentration present in the product. The data suggest that the cariostatic effect of SDF products is not fluoride-dependent.

Funded by Capes.

\section{9}

\section{Accelerated Aging Protocol to Obtain $\mathrm{MFP} / \mathrm{CaCO}_{3}-$ Based Toothpastes with Different Percentages of Insoluble Fluoride}

C.S.S. Coelho*, C.P.M. Tabchoury, J.A. Cury

csscoelhoo@gmail.com

Piracicaba Dental School, University of Campinas, Piracicaba, Brazil

Chemically soluble in a formulation toothpaste is the fraction of total fluoride (TF) potentially available to be released in the oral cavity during toothbrushing or to be absorbed in the gastrointestinal tract. MFP/CaCO${ }_{3}$-based toothpastes are not a fully chemical stable formulation and show during storage gradual reduction of total soluble fluoride (TSF $=$ MFP ion $+\mathrm{F}^{-}$ion) with concomitant increase of insoluble fluoride $\left(\mathrm{F}_{\text {ins }}\right)$. Accelerated aging protocols are useful tests to estimate the stability of fluoride in toothpaste or for research purposes. The aim of this study was to develop a protocol to obtain $\mathrm{MFP} / \mathrm{CaCO}_{3}$-based toothpaste with different percentages of $F_{\text {ins }}$ to test the relevance of bioavailable fluoride in terms benefits/risks. Fluoride concentration in fresh samples of $\mathrm{MFP} / \mathrm{CaCO}_{3}$-based toothpaste Sorriso Dentes Brancos $(1,450$ ppm of TF; Colgate $\left.{ }^{\circledR}\right)$ was determined and the tubes $(n=6)$ were kept at $55^{\circ} \mathrm{C}$ overtime. TF, TSF and ionic fluoride $\left(\mathrm{F}^{-}\right)$were chemically determined with specific electrode using standardized protocol of analysis. MFP ion and $\% \mathrm{~F}_{\text {ins }}$ were further calculated. The concentrations (mean $\pm \mathrm{SD} ; \mathrm{n}=6 ; \mu \mathrm{g} / \mathrm{g}$ ) in fresh samples and after 40 days of aging were: TF: $1447.5 \pm 48.9$ and $1509.3 \pm 12.3$; TSF: $1387.0 \pm 45.0$ and $1118.6 \pm 35.3 ; \mathrm{F}^{-}: 45.6 \pm 2.2$ and $272.7 \pm 4.2$; MFP: $1341.3 \pm 43.1$ and $845.9 \pm 31.1 ; F_{\text {ins }}=4.1$ and $21.7 \%$. The results suggest that, for this specific toothpaste, temperatures greater than $55^{\circ} \mathrm{C}$ should be tested to obtain higher percentage of $\mathrm{F}_{\text {ins }}$ in shorter times.

Funded by Funcamp 4887.1 and Capes.
100

\section{Histidine Increases the Formation of Products of} Reaction on Carious Enamel
C.P.M. Tabchoury*, L.A.S.P.F. Marques, J.A. Cury
cinthia@fop.unicamp.br
Piracicaba Dental School, UNICAMP, Piracicaba, São Paulo, Brazil

It was previously shown that histidine increases the reactivity of fluoride with carious enamel and dentine, but the mechanism of action is unknown. Thus, the aim of this study was to study whether this effect is dependent on fluoride concentration. Bovine enamel slabs ( $n=7$ /group) with caries-like lesions were randomized and submitted to treatment with fluoridated solutions containing 226 (groups 1 and 2) or $1900 \mu \mathrm{g} \mathrm{F} / \mathrm{mL}$ (groups 3 and 4). The solutions of groups 2 and 4 were buffered with $0.1 \mathrm{M}$ histidine at $\mathrm{pH} 5.0$, and the $\mathrm{pH}$ of the solutions of groups 1 and 3 was adjusted to $\mathrm{pH} 5.0$ with $\mathrm{HCl}$. Loosely bound (" $\mathrm{CaF}_{2}$ ") and firmly bound $\mathrm{F}$ (FA) formed in enamel were determined after $10 \mathrm{~min}$ of 
reaction of the slabs with the solutions. " $\mathrm{CaF}_{2}$ " was extracted with $\mathrm{KOH}$ and FA with $\mathrm{HCl}$. Two enamel layers were extracted for FA determination and the $\mathrm{F}$ concentration in both was added up. The concentrations of " $\mathrm{CaF}_{2}$ " and FA formed were analyzed by ANOVA and Tukey test. The concentration (mean $\pm \mathrm{SD} ; \mu \mathrm{g} \mathrm{F} / \mathrm{cm}^{2}$ ) of " $\mathrm{CaF}_{2}$ " in groups 1 to 4 were: $10.4 \pm 2.2 \mathrm{a} ; 23.6 \pm 3.7 \mathrm{~b} ; 135.6 \pm 9.9 \mathrm{c}$; $202.5 \pm 25.4 \mathrm{~d}$ and for FA: $1.4 \pm 0.4 \mathrm{a} ; 1.8 \pm 0.5 \mathrm{a} ; 6.0 \pm 1.4 \mathrm{~b} ; 47.0 \pm$ $24.6 \mathrm{c}$. The effect of histidine was significant $(\mathrm{p}<0.05)$, with higher concentrations of " $\mathrm{CaF}_{2}$ " (an increase of 2.3 times for $226 \mathrm{ppm} \mathrm{F}$ and 1.5 for $1900 \mathrm{ppm} \mathrm{F}$ ) and FA (an increase of 1.3 times for 226 ppm F and 7.8 for 1900 ppm F) in both concentrations of F. The results suggest that the effect of histidine, increasing fluoride reactivity with carious enamel, is independent of the concentration of fluoride.

Funded by Fapesp process number 2017/02133-9.

\section{1 \\ Fluoride Deposits Formed on Dental Surfaces and Dental Biofilm by Conventional and High-Fluoride Concentration Toothpastes}

C.E. Fernandez*, L.M.A.Tenuta, D.F. Nobrega. A.A. Del Bel Cury, J.A. Cury

cfernandez.go@gmail.com

Piracicaba Dental School, Piracicaba, São Paulo, Brazil

Evidence suggests that high-fluoride toothpastes (FT) $(5,000$ $\mathrm{ppm})$ are more effective than standard concentration FT $(1,000$ $1,500-\mathrm{ppm})$ to control dental caries. Since the mechanisms of action have not been clarified, we evaluated in situ if the superior effect of 5,000-FT may be explained by loosely-bound fluoride deposits (" $\mathrm{CaF}_{2}$ ") formed on dental slabs surface and by fluoride retained in biofilm solids. Eighteen subjects wore palatal appliances containing sound and carious enamel and dentin slabs. Placebo-F, 1,100-FT or 5,000-FT were used 2x/day during 14 days. To simulate demineralizing and remineralizing conditions, slabs/biofilms were exposed to a cariogenic challenge by dropping $20 \%$ sucrose $8 x$ /day on sound slabs or $3 x /$ day on carious ones, respectively. After each experimental phase, " $\mathrm{CaF}_{2}$ " deposited on the slabs and $\mathrm{F}$ retained in dental biofilm solids were evaluated. The effect of concentration was analyzed by linear regression. F deposited on dental substrates and in the solid portion of the biofilm increased as a function of FT concentration $(0 ; 1,100 ; 5,000)$ for all substrates/conditions $(\mathrm{p}<0.001)$. " $\mathrm{CaF}_{2}$ " $\left(\mu \mathrm{g} / \mathrm{cm}^{2}\right)$ formation ranged from $0.28 \pm 0.13$ to $1.71 \pm 1.73(0$ to $5,000-F T ; r=0.38)$ on sound-enamel; $0.36 \pm 0.49$ to $1.80 \pm 1.16$ $(\mathrm{r}=0.48)$ on carious-enamel; $0.56 \pm 0.26$ to $3.18 \pm 3.64(\mathrm{r}=0.34)$ on sound dentine; $0.41-0.16$ to $1.51 \pm 0.75(\mathrm{r}=0.53)$ on carious dentine. $\mathrm{F}$ accumulated in biofilm solids ( $\mathrm{nmol} \mathrm{F/g}$; wet weight) ranged from: $33.6 \pm 37.1$ to $254.6 \pm 275.7$ ( 0 to $5,000-\mathrm{FT} ; \mathrm{r}=0.47$ ) on sound-enamel; $42.9 \pm 75.3$ to $1052 \pm 1402(\mathrm{r}=0.47)$ on carious-enamel; $31.6 \pm 36.4$ to $322.9 \pm 459.2(\mathrm{r}=0.47)$ on sound dentine; $61.1 \pm 96.6$ to $734.8 \pm 834.1(\mathrm{r}=0.44)$ on carious dentine. Considering that " $\mathrm{CaF}_{2}$ " deposited on dental surface and $\mathrm{F}$ accumulated in the solid portion of biofilm are fluoride reservoirs, they may contribute to the superior effect of high-F concentration toothpastes to control dental caries.
This study was partially supported by FUNCAMP (Conv. 4252). The first author receiveda scholarship during this study from the National Commission for Scientific and Technological Research (CONICYT), Chile (Res. 1166/2011).

\section{2 \\ Fluoride Content in the Toothpastes Most Commonly Used in Households of the ELEMENT-Cohort in Mexico}

G.A. Castiblanco*a, C. Buckley ${ }^{\mathrm{a}}$, G.J. Eckert ${ }^{\mathrm{b}}$, F. Lippert ${ }^{\mathrm{a}}$, N. Basuc, A. Benito ${ }^{d}$, A. Cantoral ${ }^{d}$, A. Mercado-Garcia ${ }^{\text {, }}$,

M. Hernandez-Avila ${ }^{\mathrm{d}}, H$. Hu ${ }^{\mathrm{e}}$, B. Sanchez ${ }^{\mathrm{f}}$, M. Tellez-Rojo ${ }^{\mathrm{d}}$, K.E. Peterson' ${ }^{9}$, E.A. Martinez-Mier

ginacast@iu.edu

aDepartment of Cariology, Operative Dentistry and Dental Public Health, Indiana University School of Dentistry, Indianapolis, Indiana, USA; bepartment of Biostatistics, Indiana University School of Medicine, Indianapolis, Indiana, USA; 'School of Dietetics and Human Nutrition, McGill University, Montreal, Canada; ${ }^{\mathrm{N}}$ National Institute of Public Health, Mexico City, Mexico; ' Dalla Lana School of Public Health University of Toronto, Toronto, Ontario, Canada; fDepartment of Biostatistics, University of Michigan School of Public Health, Ann Arbor, Michigan, USA; ' Department of Environmental Health Sciences, University of Michigan School of Public Health, Ann Arbor, Michigan, USA

We determined total fluoride content in the five toothpaste formulations most commonly used by households with child/ adolescent-mother pairs participating in cohorts of the Early Life Exposures in Mexico to Environmental Toxicants study (ELEMENT), collected as part of a cross-sectional market-basket study. Child/adolescents were 9-to-19-year-olds at the time of sample collection. We also tested toothpaste-compliance with labeled total fluoride (1450 $\mu \mathrm{g}$ F-/g) and adherence to Mexican regulations $(\leq 1500 \mu \mathrm{g} \mathrm{F-/g).} \mathrm{A} \mathrm{sample} \mathrm{size} \mathrm{of} 34$ per formulation $(\mathrm{n}=$ 170) was determined to be sufficient to test differences $(80 \%$ power, two-sided t-test, $\alpha=0.05$ ). A study nurse visited the home of participants and transferred a sample of toothpaste from the original container to vials that were shipped to the laboratory. Toothpaste slurries (1:100 dilution) were prepared and the ADA Standard Test Method 1 (microdiffusion) and 2a (direct) were utilized for sodium monofluorophosphate- $(\mathrm{n}=1)$ and sodium fluoride-containing formulations $(\mathrm{n}=4)$, respectively. Standard curves were prepared for each test method and used for determination of the fluoride content. Fluoride was measured with an ion-selective electrode coupled to a $\mathrm{pH} / \mathrm{ISE}$ meter. Descriptive statistics and two-sided t-tests $(\alpha=0.05)$ were performed. Results for total fluoride ( $\mu \mathrm{g}$ F-/g;mean $\pm \mathrm{SD}$ ) in sodium fluoride formulations were: Colgate Triple Acción (1568.4 \pm 41.5$)$, Crest Complete $(1431.1 \pm 300.4)$, Colgate Total $12(1550.1 \pm 108.9)$, Colgate MaxFresh (1523.8 \pm 71.5$)$, and for the sodium monofluorophosphate formulation, Colgate Máxima Protección Anticaries, $(1442.3 \pm 157.6)$. We found that three out of four sodium fluoride formulations had fluoride levels significantly higher than stated 
on the label ( $p<0.05)$. Furthermore, at least $59 \%$ of fluoride levels of samples from sodium fluoride and $38 \%$ of the monofluorophosphate formulations were above the allowed threshold of $\leq 1500 \mu \mathrm{g} \mathrm{F}-/ \mathrm{g}$. These results provide a cross-sectional estimate of fluoride content of toothpastes representative of the ELEMENT cohort.

Funded by NIH RO1ES021446: Prenatal and Childhood Exposure to fluoride and Neurodevelopment and the Department of Cariology, Operative Dentistry and Dental Public Health, Indiana University School of Dentistry.

\section{3 \\ Comparison of Three Methods for Analysing Fluoride in Microlitre-Volume Plasma Samples}

F.V.Zohoori*a, A. Martinez-Mier ${ }^{\text {b }}$, A. Maguire ${ }^{c}$, M. Buzalaf ${ }^{\text {, }}$, R. Sanderson ${ }^{\mathrm{e}}$, G.J. Eckert ${ }^{\mathrm{b}}$

v.zohoori@tees.ac.uk

aSchool of Health and Social Care, Teesside University,

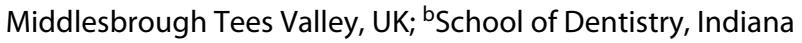
University, US; 'C Centre for Oral Health Research (COHR), School of Dental Sciences, Newcastle University, Newcastle upon Tyne, UK; ${ }^{\mathrm{d} B a u r u}$ School of Dentistry, University of São Paulo, São Paulo, Brazil; e'School of Natural \& Environmental Science, Newcastle University, Newcastle upon Tyne, UK

The aim was to compare potential methods for fluoride analysis in microlitre-volume plasma samples containing nano-gram amounts of fluoride. A group of four laboratories analysed a set of standardised samples as well as plasma to determine fluoride concentration using three methods. In Phase 1, fluoride analysis was carried out using the established HMDS-diffusion method (1 ml-aliquot/analysis) [Martinez-Mier et al.: Caries Res 2011;45:3-12] to obtain preliminary measurement of agreement between the laboratories. In Phase 2, the laboratories analysed the same samples using a micro-diffusion method [Martinez-Mier et al.: J Dent Res 2010;89(Spec IssB):931] and known-addition technique [Ekstrand J.: Calc Tiss Res 1977;23:225-228] with $200 \mu \mathrm{l}$-aliquot/analysis. Coefficients of Variation (CVs) and intra-class correlation coefficients (ICCs) were estimated using ANOVA to evaluate the amount of variation within- and between-laboratories. Based on the results of the Phase 2 analysis, 20 human plasma samples were analysed and compared using the HMDS-diffusion method and known-addition technique in Phase 3. Comparison of Phase 1 results showed no statistically significant difference among the laboratories for the overall data set. The mean between- and within-laboratory CVs and ICCs were $<0.13$ and $<0.99$, respectively, indicating very low variability and excellent reliability. In Phase 2 , the overall results for between-laboratory variability showed a poor CV (1.16) and ICC $(0.44)$ for the micro-diffusion method, whereas the corresponding values were 0.49 and 0.83 with the known-addition technique. Phase 3 results showed no statistically significant difference in fluoride concentrations of the plasma samples measured with HMDS-diffusion method and known-addition technique with a mean (SE) difference of $0.002(0.003) \mu \mathrm{g} / \mathrm{ml}$. In conclusion the known-addition technique could be an alternative option for measurement of fluoride in plasma with microlitre-volume samples.

This study was supported by a grant from The Borrow Foundation.

\author{
104 \\ Fluoride Concentration in Toothpastes from the \\ Philippines Sold in Sachets \\ W. Plett*a, A.G. Schulte ${ }^{\text {b }}$ \\ walterplett@gmx.de \\ aDepartment of Conservative Dentistry, University of \\ Heidelberg, Heidelberg, Germany; bepartment for Special \\ Care Dentistry, Witten/Herdecke University, Witten, Germany
}

In a previous study we found that some of the toothpastes sold in the capital of the Philippines had low F-concentrations. Interestingly, in the Philippines, toothpastes are available not only in tubes but also in sachets which contain a lower amount of toothpaste and are cheaper than tubes. So far, no study presenting data on F-concentration in toothpastes sold in sachets has been published. The aim of this study was to determine the F-concentration of toothpastes sold in sachets or tubes in a remote area of the Philippines. In street markets of two small islands in the Philippines (Camiguin and Siquijor) 27 different toothpastes were purchased randomly. Out of these 11 were packed in tubes and 16 in sachets. All were labeled to contain either $\mathrm{NaF}$ or NaMFP or NaMFP and NaF. In the laboratory of the University of Heidelberg Dental School Fconcentrations were measured in $\mathrm{mV}$ with the aid of an F-specific electrode by applying the $\mathrm{HCl}$-method [Cury et al.: Braz Dent J 2010; 21:396-400] and the AcP-method [Van Loveren et al.: Caries Res 2005; 39:224-230] for all samples. The program "GraphPad Prism“ (Version 7, GraphPad Software, Inc. La Jolla, USA) was used to transfer the F-values from $\mathrm{mV}$ into $\mathrm{mg} / \mathrm{kg}$ by taking into account the values of the calibration curve. For the statistical analysis the Mann-Whitney Test was used and p-values $<0.05$ were set as statistically significant. In sachets, the medians of F-concentrations $(\mathrm{HCl} / \mathrm{AcP})$ were $1377 / 1308 \mathrm{mg} / \mathrm{kg}$ with a 0.25 -quartile of $1004 / 943 \mathrm{mg} / \mathrm{kg}$ and a 0.75 -quartile of $1450 / 1440 \mathrm{mg} / \mathrm{kg}$. In tubes, a statisically significant lower F-concentration was found ( $\mathrm{p}=$ 0.006 for the $\mathrm{HCl}$-method and $\mathrm{p}=0.03$ for the AcP-method). Here the medians of F- concentrations (Hcl/AcP) were $894 / 830 \mathrm{mg} / \mathrm{kg}$ with a 0.25 -quartile of $750 / 621 \mathrm{mg} / \mathrm{kg}$ and a 0.75 -quartile of $1134 / 1151 \mathrm{mg} / \mathrm{kg}$. So far there is no explanation why toothpastes sold in sachets contain more fluoride than those sold in tubes. Toothpastes available in sachets should be considered in further studies and its quality and frequency of usage should be investigated in comparison to toothpastes in tubes.

Funded by the Department of Conservative Dentistry of Heidelberg University Dental School, Heidelberg, GermanyAcademic foundation "Studienstiftung des Deutschen Volkes". 
105

\section{Fluoride Content in Mineral Waters from Germany}

P. Schmidt*a, E.A. Naumova ${ }^{\text {b }}$, A.G. Schulte ${ }^{\text {a }}$

peter.schmidt@uni-wh.de

aDepartment of Special Care Dentistry, Dental School, Witten/

Herdecke University, Witten, Germany; ${ }^{\text {b}}$ Department of

Biological and Material Sciences in Dentistry, Faculty of Health,

Witten/Herdecke University, Witten, Germany

According to the German Order for Mineral Waters (GOM) companies which bottle mineral water from natural sources are not obliged to label the fluoride content but have to print the term "contains fluoride" as soon as the F-concentration exceeds $1 \mathrm{mg} / \mathrm{L}$. It is allowed to label the mineral water as "applicable for the preparation of baby food" when the F-concentration of the mineral water is $<0.7 \mathrm{mg} / \mathrm{L}$. This regulation makes it difficult in Germany to take a complete fluoride exposure history. The aim of the study was to measure the F-concentration in mineral waters available in German supermarkets. In the year 2017, bottles with different mineral waters were bought in several German regions. The Fconcentration was measured in $\mathrm{mV}$ with the aid of an F-selective electrode (Thermo Scientific 9609BNWP) after having mixed a sample of $5 \mathrm{ml}$ with Low-Level-TISAB. The program "GraphPad Prism" (Version 7, GraphPad Software, Inc. La Jolla, USA) was used to transfer the F-values from $\mathrm{mV} \mathrm{F}$-values to $\mathrm{mg} / \mathrm{L}$ by taking into account the values of the calibration curve and to determine the mean values. In total, 111 brands of mineral water from $75 \mathrm{Eu}$ ropean sources (69 sources from Germany) were included. The mean F-concentration of all brands was $0.26 \mathrm{mg} / \mathrm{l}$ (SD 0.2, range $0.05-1.21 \mathrm{mg} / \mathrm{l})$. The mean value of the brands which labeled the F-concentration $(\mathrm{n}=43)$ was $0.30 \mathrm{mg} / \mathrm{l}(95 \%$ CI $0.18-0.33)$ while the respective values according to the labels were $0.31 \mathrm{mg} / \mathrm{l}(95 \%$ CI 0.19-0.35). The mean F-concentration of the mineral waters without labeling this value was $0.23 \mathrm{mg} / \mathrm{l}$ (95\% CI $0.19-0.28)$. In 104 brands (93.6\%) the F-concentration was below $0.7 \mathrm{mg} / \mathrm{l}$ and in one case beyond $1.0 \mathrm{mg} / \mathrm{l}$. In general, mineral waters from German sources do not represent an important source for the intake of fluoride. According to the GOM and regarding the F-concentration the majority of these mineral waters are applicable for the preparation of baby food. In order to make the fluoride content of mineral waters more transparent it is recommended to change the GOM so that producers will be obliged to label the actual F-concentration.

This study was funded by the Department of Special Care Dentistry and by the Department of Biological and Material Sciences in Dentistry of the Witten/Herdecke University.

\section{6 \\ Mineral Distribution Within Oral Biofilm Which Was Grown on Mineral-Releasing Composite Resin Containing S-PRG filler}

K. Kato*, K. Tamura, Y. Shimazaki

kazkato@dpc.aichi-gakuin.ac.jp

School of Dentistry, Aichi Gakuin University, Nagoya, Japan

A three-way crossover trial using depth-specific analysis was conducted to estimate the distribution of mineral ions released from composite resin (CR) containing surface pre-reacted glassionomer (S-PRG) filler within the oral biofilms grown on the CR. Three kinds of CR disks were prepared; "S-PRG" disk contained $70 \mathrm{wt} \%$ of S-PRG fillers which releases several ions (Al, B, F, Si, Sr), positive control (PC) disk contained $3.2 \% \mathrm{NaF}$ which could release the same level of fluoride as "S-PRG" disk, and negative control (NC) disk contained no active ingredients. Sixteen healthy participants wore in situ plaque-generating devices (a pair of $4 \mathrm{~mm}$ diameter disks) on their upper molars, and biofilm was allowed to form for four days. Devices were collected and samples were serially sectioned, with $2 \times 2-\mu \mathrm{m}$ sections taken first, followed by $44-\mu \mathrm{m}$ sections for separation into outer, middle and inner layered fractions $(300 \mu \mathrm{m}$ thick). This procedure was repeated until the required thickness was obtained. Fluoride and the four other mineral ions extracted from the thicker sections were quantified using a fluoride-selective electrode and ICP-atomic emission spectroscopy, respectively. The results were corrected for biomass volume estimated by area measurement of stained $2-\mu \mathrm{m}$ sections. Detected levels $\left(\mathrm{ng} / \mathrm{mm}^{3}\right)$ of $\mathrm{Al}, \mathrm{B}, \mathrm{F}, \mathrm{Si}$ and $\mathrm{Sr}$ within layered samples were within the following ranges: S-PRG) 51.8-142.6, 70.2-74.5, 4.6$16.6,14.8-16.7$ and 55.3-77.8, PC) 8.2-26.6, 0, 23.0-59.3, 1.3-10.3 and 14.9-20.3, NC) 9.2-13.4, 0, 1.5-1.8, 10.0-32.9 and 29.3-43.6. Although the same levels of fluoride were expected between S-PRG and PC samples, only PC contained significantly higher fluoride than NC. These results suggest that fluoride behavior within oral biofilm might be effected when fluoride is applied at the same time as other mineral ions released from the same resources.

This study was supported by KAKENHI (C) No. 15K11437, from the Japan Society for the Promotion of Science.

\section{7 \\ Acid Treatment to Determine Fluoride Concentration in NaF/Silica-Based Toothpastes}

\author{
J.A. Cury*, A.P. Ricomini-Filho, C.P.M. Tabchoury \\ jcury@unicamp.br \\ Piracicaba Dental School, University of Campinas, Piracicaba, \\ Brazil
}

Hydrochloric acid has been used to determine total fluoride (TF) and total soluble fluoride concentrations in toothpastes [Cury et al.: Rev APCD 1981;35:142-7] and its role is to hydrolyze MFP (monofluorphosphate ion) and to dissolve insoluble fluoride salts bound to abrasive in $\mathrm{MFP} / \mathrm{CaCO}_{3}$-based toothpastes. However, acid treatment is not necessary to analyze $\mathrm{NaF} / \mathrm{Silica}$-based tooth- 65th ORCA Congress 
pastes and in addition the concentration could be underestimated due to the loss of hydrofluoric acid $\left(\mathrm{BP}=19.5^{\circ} \mathrm{C}\right)$. We evaluated $\mathrm{TF}$ concentration in $\mathrm{NaF} /$ Silica-based toothpastes by analyses made with (Ac+) or without (Ac-) acid pre-treatment. TF concentration was determined in 3 tubes of 7 brands of NaF/Silica-based toothpastes $(n=21)$. The declared TF concentration in the label of the toothpastes ranged from 1,000 to $1,450 \mathrm{ppm} \mathrm{F}$ ( $\mu \mathrm{g} \mathrm{F} / \mathrm{g}$; w/w). One hundred milligrams of each toothpaste tube was homogenized in $10.0 \mathrm{~mL}$ of purified water. Duplicates of the volumes of 0.25 and $1.0 \mathrm{~mL}$ of the suspension were transferred to tubes marked TF-Ac+ and TF-Ac-, respectively. To the tubes TF-Ac+, $0.25 \mathrm{~mL}$ of $2.0 \mathrm{M} \mathrm{HCL}$ was added and after $1 \mathrm{~h}$ at $45^{\circ} \mathrm{C}$ the extracts were buffered with $0.50 \mathrm{~mL}$ of $1.0 \mathrm{M} \mathrm{NaOH}$ plus $1.0 \mathrm{~mL}$ of TISAB II [Cury et al.: Braz Dent J 2010;21:396-400]. To the tubes TF-Ac-, only $1.0 \mathrm{~mL}$ of TISAB II was added. Fluoride was analyzed with specific electrode calibrated with fluoride standard solutions prepared in the same conditions that the samples (Ac+ or Ac-). The concentrations found (mean $\pm \mathrm{SD} ; \mathrm{n}=7$ ) of $971.3 \pm 191.2$ and $982.4 \pm 201.3 \mu \mathrm{g} \mathrm{F} / \mathrm{g}$, respectively for the analyses Ac+ and Ac-, did not differ statistically ( $\mathrm{p}=0.22$, $\mathrm{t}$ test). The finding confirms that the determination of fluoride in $\mathrm{NaF} / \mathrm{Silica}$-based toothpastes can be made using the same protocol to analyze $\mathrm{MFP} / \mathrm{CaCO}_{3}$-based toothpastes.

This study was funded by Funcamp (Proc 4887.1).

\section{8 \\ Potential of Human Milk to Provoke Enamel Demineralization \\ M.G.B. Aragão*a , A.P. Ricomini-Filho ${ }^{\mathrm{a}}$, A.B. Alvarado ${ }^{\mathrm{a}}$, A.A. Del Bel Cury ${ }^{\mathrm{a}}$, C.S.S. Coelho ${ }^{\mathrm{a}}$, A.V.L. Bandeira ${ }^{\mathrm{b}}$, A.M.C. Leal ${ }^{\mathrm{b}}$, C.P.M. Tabchoury a, J.A. Cury ${ }^{\mathrm{a}}$ \\ magebrito.wku@gmail.com \\ apiracicaba Dental School, University of Campinas, Piracicaba, Brazil; ${ }^{\text {}}$ Federal University of Piaui, Teresina, Brazil}

Human milk as a risk factor for caries is an issue in debate, although lactose has not the cariogenic properties of sucrose, the most cariogenic dietary sugar. Thus, we evaluated if human milk fermentation is able to provoke in situ enamel demineralization. The study had a 4-phase crossover, double blind design in which eight volunteers wore palatal appliances containing four bovine enamel blocks of known surface hardness ( $\mathrm{SH})$. The blocks were covered with a test plaque of Streptococcus mutans Ingbritt-1600 and placed in palatal appliances. The volunteers wore the appliance for $30 \mathrm{~min}$. After that, in each phase, the appliances were immersed extra-orally for $5 \mathrm{~min}$ into one of the following treatments: $0.9 \% \mathrm{NaCl}$ solution (negative control), human milk (test), $7 \%$ lactose solution (active control) or 7\% sucrose solution (positive control). The appliances were replaced into the mouth and after 45 min the enamel blocks were collected. The demineralization provoked was determined by the percentage of SH loss (\%SHL) in relation to baseline. Data were analyzed by ANOVA one-way and Tukey's test $(\alpha=5 \%)$. The \%SHL of the groups human milk and $7 \%$ lactose did not differ from the negative control ( $p>0.05)$, being all the values under $6.8 \%$. However, for the $7 \%$ sucrose group, the $\%$ SHL differed from all groups $(\mathrm{p}<0.05)$ reaching a maximum of $29.8 \%$. The findings, using this short-term in situ model, suggest that human milk does not present a potential to demineralize enamel.

This study was funded by Funcamp (Proc 4887.1).

\section{9 \\ Effect of Linoleic Acid on the Competition Between Streptococcus mutans and Streptococcus sanguinis in a Caries-Biofilm Model}

\author{
R.A. Giacaman*, M.J. Valenzuela-Verdugo, H. Vera-Bueno, \\ N. Díaz-Garrido. \\ giacaman@utalca.cl \\ Cariology Unit, Department of Oral Rehabilitation and \\ Interdisciplinary Excellence Research Program on Healthy \\ Aging (PIEI-ES), University of Talca, Talca, Chile
}

We previously reported an anticaries effect of the polyunsaturated linoleic acid. In the context of the current ecological conception of caries, how this fatty acid may create environmental conditions for the development of healthy biofilms has not been reported. The aim was to study the consequences of linoleic acid exposure on the competition between a commensal and a cariogenic species of the dental biofilm, under a cariogenic environment. A dualspecies caries-biofilm model with Streptococcus mutans UA159 (Sm) and Streptococcus sanguinis SK36 (Ss) was used. Saliva-coated enamel slabs were inoculated with equal amounts (1:1) of cells $\left(1 \times 10^{8} \mathrm{CFU} / \mathrm{mL}\right)$ for both bacteria and distributed in 4 groups by inoculation sequences: 1) Ss followed by Sm (Ss-Sm), 2) Sm and Ss inoculated at the same time $(\mathrm{Sm}=\mathrm{Ss})$ and the controls 3) Sm followed by $\mathrm{Sm}(\mathrm{Sm}-\mathrm{Sm})$ and 4$)$ Ss followed by Ss (Ss-Ss). Mature dual-species biofilms were exposed $3 \mathrm{x} /$ day to $10 \%$ sucrose followed by either $100 \mathrm{mM}$ linoleic acid, $0.05 \% \mathrm{NaF}$ or $0.9 \% \mathrm{NaCl}$ (caries-positive control). Acidogenicity and demineralization by microhardness were assessed and biofilms analyzed for biomass and viable cells. Triplicates for each condition in two independent experiments were assayed $(n=6)$ and analyzed by ANOVA followed by post-hoc at $\mathrm{p}$-value $<0.05$. Linoleic acid exposure after sucrose resulted in the lowest acidogenicity and demineralization in all inoculation sequences. The polyunsaturated fatty acid significantly reduced demineralization from $49.9 \%, 33.9 \%, 33.6 \%$ and $46.3 \%$ to $3.2 \%, 2.2 \%, 2.2 \%$ and $3.6 \%$ for Sm-Sm, Ss-Ss, Ss-Sm and $\mathrm{Ss}=\mathrm{Sm}$, respectively $(\mathrm{p}<0.05)$. Sucrose-induced demineralization in the linoleic acid groups was lower than that exhibited by $0.5 \%$ $\mathrm{NaF}$ and only slightly higher than the caries-negative control $(\mathrm{p}<$ 0.05 ). Although biomass and viable microorganisms were lower in all inoculation sequences when compared to Sm-Sm, no significant differences were detected across the treatment groups, suggesting a metabolic rather than an antibacterial effect. In conclusion, linoleic acid seems to greatly reduce the cariogenic potential of sucrose and appears to create favorable conditions for the onset of commensal biofilms.

Funded by the Chilean Government Grant Fondecyt \#1140623 to RAG. 


\section{0}

\section{Antimicrobial Efficacy of Natural Antimicrobials Towards Biofilms from Cariogenic Bacteria In Vitro}

\author{
F. Cieplik*a ,E. Kara ${ }^{\text {a }}$ D. Muehler ${ }^{\text {a }}$, J. Enax ${ }^{\text {b }}$, K.-A. Hillera, \\ T. Maisch', W. Buchalla ${ }^{\text {a }}$
}

fabian.cieplik@ukr.de

aDepartment of Conservative Dentistry and Periodontology, University Medical Center Regensburg, Regensburg, Germany;

${ }^{b}$ Research Department Oral Care, Dr. Kurt Wolff GmbH \&

Co. KG, Bielefeld, Germany; 'Department of Dermatology,

University Medical Center Regensburg, Regensburg, Germany

For caries-active patients, antimicrobial measures may be useful in addition to mechanical biofilm-removal. Hereby, the use of natural antimicrobials may be a biomimetic option. This study investigates the antimicrobial efficacy of citrus-extract and four essential oils (EOs) as compared to gold-standards chlorhexidine (CHX) and cetylpyridinium chloride (CPC) towards biofilms in vitro. Monospecies-biofilms from Streptococcus mutans (DSM20523) and polymicrobial biofilms from Actinomyces naeslundii (DSM-43013), Actinomyces odontolyticus (DSM-19120) and S. mutans were formed under anaerobic conditions in 96-well-plates for total culture periods of 24 or $72 \mathrm{~h}$ employing a modified Zurich biofilm culture broth. First, the antimicrobial efficacy of all compounds was screened in $24 \mathrm{~h} \mathrm{~S}$. mutans biofilms (treatment period: $10 \mathrm{~min}$ ). Then, the most effective compounds were further investigated in various concentrations in $72 \mathrm{~h}$ polymicrobial biofilms (treatment period: $10 \mathrm{~min}$ ). Antimicrobial efficacy was evaluated by colony-forming-units ( $\mathrm{CFU} ; \mathrm{n}=12$ ). For polymicrobial biofilms, bacterial species were differentiated according to their respective colony morphology. Additionally, biofilms were exemplarily visualized by scanning electron microscopy (SEM; highvacuum-mode). All four EOs showed $<2 \log 10$ reduction of CFU in $S$. mutans biofilms after treatment for $10 \mathrm{~min}$. In contrast, citrus-extract exhibited $\geq 5 \log 10$ and was therefore tested further in polymicrobial biofilms. In polymicrobial biofilms, all compounds showed concentration-dependent efficacy: $\mathrm{CHX}$ was the most effective compound ( $\geq 5 \log 10$ at $0.2 \%)$ followed by CPC ( $\geq 4 \log 10$ at $0.1 \%)$. Citrus-extract exhibited $\geq 1 \log 10$ reduction of CFU at $0.25 \%$. SEM showed debris on the top layers of the biofilms after treatment with CHX and CPC, most likely originating from dead cells. Although no tested substance was as effective as gold-standards CHX and CPC, citrus-extract may still be a valuable alternative for use in oral care.

This work was funded in part by Dr. Kurt Wolff GmbH \& Co. KG, Bielefeld, Germany.

\section{1 \\ Cariogenic Potential of Human and Bovine Milk and Its Effect on Enamel Demineralization}

\author{
A.P. Ricomini-Filho*, A.C.M. de Assis, B.E. Costa-Oliveira, J.A. Cury \\ ricomini@unicamp.br \\ Piracicaba Dental School, University of Campinas, Piracicaba, \\ Brazil
}

The cariogenicity of milk is a controversial issue and in addition human milk (HM) has been considered more cariogenic than bovine milk (BM). Therefore, we evaluated the effect of HM and $\mathrm{BM}$ on biofilm composition and enamel demineralization using a cariogenic biofilm model. Streptococcus mutans UA159 biofilms $(\mathrm{n}=8)$ were grown on human saliva-coated bovine enamel slabs of known surface hardness $(\mathrm{SH})$. The biofilms were grown in UTYEB medium ( $\mathrm{pH} 7.0$ ) and were exposed $8 \mathrm{x} /$ day to $0.9 \%-\mathrm{NaCl}$ solution (negative control), human milk (HM), bovine milk (BM), 7\%-lactose solution (HM-control), 4.5\%-lactose solution (BMcontrol) or $10 \%$ sucrose solution (positive control). The culture medium was changed twice daily and the $\mathrm{pH}$ was analyzed. After 5 days of formation, biofilms were collected to evaluate $\mathrm{CFU}$ counts and extracellular polysaccharides (EPS), soluble and insoluble. Enamel slabs were used to quantify the percentage of enamel SH loss (\%SHL). Data were analyzed by ANOVA one-way and Tukey's test, or t-test $(\alpha=5 \%)$. Only the sucrose group presented lower $\mathrm{pH}$ values than the negative control for all time points after $32 \mathrm{~h}$ of biofilm growth $(\mathrm{p}<0.05)$. The sucrose group was also the only to show higher $(\mathrm{p}<0.05)$ bacterial counts $(9.4 \pm 0.2 ; \log 10)$ in comparison to the negative control group $(7.8 \pm 0.6)$. Soluble and insoluble EPS were only formed in the biofilm exposed to sucrose, $19.5 \pm 10.8$ and $164.0 \pm 12.6 \mu \mathrm{g} / \mathrm{biofilm}$, respectively. The $\% \mathrm{SHL}$ values for the groups HM (7.5 \pm 5.0$), \mathrm{BM}(8.7 \pm 6.3)$, HM-control $(13.3 \pm 7.5)$ and BM-control $(15.3 \pm 8.2)$ did not differ from the negative control $(7.7 \pm 3.1)(p>0.05)$, and sucrose presented the highest enamel demineraliztion $(55.1 \pm 5.4)(\mathrm{p}<0.05)$. The findings suggest that $\mathrm{HM}$ and $\mathrm{BM}$ have low cariogenic potential in terms of biofilm composition and they are not able to provoke enamel demineralization in comparison with sucrose in this model with this short exposure period.

This study was funded by Funcamp (Proc 4887.1). 


\section{2 \\ The Inhibitory Role of $\boldsymbol{W}$. cibaria CMU on Dental Biofilm Formation: In Clinical Studies}

A.O. Kim*a, H.J. Kim ${ }^{\text {b }}$, H.R. Park

siki-s@daum.net

aDepartment of Preventive and Public Health Dentistry, Chonnam National University of Gwangju, Gwangju, Republic of Korea; ${ }^{b}$ Department of Pediatric Dentistry, School of Dentistry, Chonnam National-University of Gwangju, Gwangju, Republic of Korea; 'Division of Liberal Arts and Teacher Training, Kwangju Women University of Gwangju, Gwangju, Republic of Korea

Weissella cibaria has been found in Korean kimchi fermentation and other sources, including fermented foods such as sausages, Greece salami, and healthy human infants. We attempted to verify the possibility of inhibition of dental biofilm formation on the enamel surface by using lactic acid bacteria of $W$. cibaria CMU. As a measuring method, modified patient hygiene performance index (m-PHP index) was measured to quantify oral hygiene status among 3 subject groups consisting of ora CMU, a commercial product containing W. cibaria CMU, the same commercial product but removing W. cibaria CMU as a placebo control, and ProDentis (BioGaia ProDentisTM) as a positive control. Each group had 30 subjects. Study subjects are required to diet an aforementioned product for 4 weeks. The measurement of m-PHP index was performed every 2 weeks starting from the first day of the experiment. Also, to know the effects of after-diets, its measurements were continued every 2 weeks for 4 weeks. The analysis of m-PHP index measurements showed 38.2 ( 0 week), 37.6 ( 2 week), 32.8 (4 week) for ora CMU, with the m-PHP index decreasing. Meanwhile, the m-PHP index of the placebo control showed 35.6 (0 week), 45.0 ( 2 week), 36.0 ( 4 week) with its index relatively increasing. In particular, there was a statistically significant difference $(\mathrm{p}<0.05)$ between ora CMU and placebo control groups during both of the 2-week and 4-week. Also, statistical significances between the ora CMU and ProDentis groups during both of the 2 -week and 4-week were found when the m-PHP indexes were compared. Taken together, our results demonstrated that only ora CMU diet could suppress biofilm formation on the enamel surface in oral hygiene.

Funded by Oradentics Research Institute.

\section{3 \\ Energy Dispersive X-Ray Spectroscopy Can Measure pH and Cation Dependent Enamel Fluoride Precipitation}

\author{
K.J. Scholz*, M. Federlin, H. Ebensberger, G. Ferstl, K.-A. Hiller, \\ W. Buchalla
}

\section{Konstantin.Scholz@ukr.de}

Department of Conservative Dentistry and Periodontology, University Medical Center Regensburg, Regensburg, Germany

This in vitro study investigates (A) energy dispersive $\mathrm{x}$-ray spectroscopy (EDX) as a semiquantitative analytical method to detect enamel fluoride precipitation (B), with/without pellicle, of $\mathrm{NaF}$ in acidic and neutral gelbase, (C) compared to an acidic amine-fluoride/ $\mathrm{NaF}$ combination. Crowns of 25 freshly extracted human caries-free third molars were quartered vertically and the enamel ground flat (FEPA 4000). Two quarters each were stored in human saliva (120 min pellicle formation). Teeth were randomly allocated to 5 treatment groups $(n=5)$ : Elmex_a (acidic amine and sodium fluoride; Elmex Geleé, CP-GABA GmbH, 12500 $\mathrm{ppmF}^{-}, \mathrm{pH}$ 4.75), NaF_a (acidic sodium fluoride; $12500 \mathrm{ppmF}^{-}$, pH4.75), NaF_n (neutral sodium fluoride; $12500 \mathrm{ppmF}^{-}, \mathrm{pH} 7.0$ ), GB_a (acidic gel base; 0 ppmF $^{-}$, pH4.75), GB_n (neutral gel base; $0 \mathrm{ppmF}^{-}, \mathrm{pH} 7.0$ ). Each gel was applied to one quarter with and one quarter without pellicle, left for 60 seconds and removed using deionized water. Two quarters served as controls (no gel, with/without pellicle). Atomic percent fluorine $(\mathrm{At} \% \mathrm{~F})$ was measured consecutively (EDX; FEI Quanta 400 FEG, EDAX micro analysis system, TEAM Enhanced v.4.3, $10^{-4}-10^{-6}$ Torr, $10 \mathrm{kV}$, spot $5-6$, $\mathrm{WD}=10 / 12 \mathrm{~mm}$, ap 4 ; non-parametrical statistics, $\alpha=0.05)$. Without pellicle [At\%F median, (25-75\% percentile)]: Elmex_a 12.6 (9.9-15.4); NaF_a 11.8 (10.9-15.0); NaF_n 0 (0-0.1); GB_a 0 (00); GB_n 0 (0-0). With pellicle: Elmex_a 7.4 (7.1-12.9); NaF_a 9.9 (9.0-10.3); NaF_n 0.1 (0.1-0.2); GB_a 0 (0-0); GB_n 0 (0-0). No At\%F was found using gels without fluoride or no gel, with/without pellicle. Differences between acidic $\mathrm{NaF}$ and amine-NaF combination (Elmex) were not significant. Pellicle had significant influence on At $\% \mathrm{~F}$ in NaF_a $(\mathrm{p}=0.016)$. In conclusion, (A) EDX can measure enamel fluoride precipitation influenced by $\mathrm{pH}$ and pellicle semiquantitatively in vitro. (B) Fluoride precipitation was generally lower with pellicle present. (C) Low $\mathrm{pH}$-values increased fluoride precipitation significantly.

The authors are grateful to Chemische Fabrik Kreussler \& Co. $\mathrm{GmbH}$ (Wiesbaden, Germany) for supporting this investigation. 


\section{4}

\section{Short-Term Effects of Preventive Applications on Salivary Parameters in Mentally and Psychosocially Handicapped Children}

Z.C. Pehlivan-Celik*a, B. Tarcin' ${ }^{\text {a }}$ S. Peker ${ }^{\text {b }}$, O. Hayran', B. Alev-Tuzuner ${ }^{\mathrm{d}}$, A. Yarat ${ }^{\mathrm{d}}$

zcerenpehlivan@gmail.com

aDepartment of Restorative Dentistry, Faculty of Dentistry, Marmara University, Istanbul, Turkey; ${ }^{b}$ Department of Pediatric Dentistry, Faculty of Dentistry, Marmara University, Istanbul, Turkey; ' ${ }^{\text {Department }}$ of Public Health, Medical School of

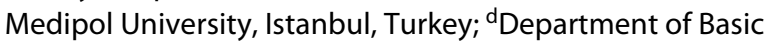
Medical Sciences, Biochemistry, Faculty of Dentistry, Marmara University, Istanbul, Turkey

Mental Retardation (MR) and Attention Deficit Hyperactivity Disorder (ADHD) are two main chronic neurodevelopmental disorders diagnosed in childhood with periods of exacerbations. This study aims to investigate the short-term effects of preventive applications such as topical fluoride and Oral Hygiene Instruction (OHI) on salivary parameters of these children. Forty-five disabled children aged 4-12 with ADHD $(n=20)$ or MR $(n=25)$ was included in the study. Detailed medical history and DMFT values were recorded after informed consent was taken from the parents. Prior preventive applications, unstimulated saliva samples were collected 2 hours after the breakfast. At the end of one month sampling procedures was repeated. All samples were kept at $-20^{\circ} \mathrm{C}$ until analyzed. Before analysis samples were thawed and centrifuged for 10 minutes at $4000 \mathrm{rpm}$. Saliva $\mathrm{pH}$ was measured by $\mathrm{pH}$ meter and the buffering capacity by the Ericsson method. $600 \mu \mathrm{L}$ of $\mathrm{HCl}(0.0033 \mathrm{M})$ was added to $200 \mu \mathrm{L}$ saliva sample and mixed by vortexing. After incubated at room temperature for $10 \mathrm{~min}$. the $\mathrm{pH}$ of the samples was measured again. Data were analyzed by $\mathrm{X}^{2}$ test and Pearson's correlation analysis. The mean DMFT of all children was 4.6. There were significant positive correlations between initial $\mathrm{pH}$ and buffering capacity $(\mathrm{r}=0.343 ; \mathrm{p}=0.021)$, and measured $\mathrm{pH}$ and buffering capacity following preventive applications $(\mathrm{r}=$ $0.398 ; \mathrm{p}=0.007$ ). Final buffering capacity of both MR and ADHD children significantly increased among the groups $62.5 \%$ and $62.1 \%$ respectively and the difference between two groups was not statistically significant. Exacerbation periods of mental disorders may increase the risk of new developing caries. Minor preventive precautions, especially $\mathrm{OHI}$ and parent motivation are advisable.

This project was funded by The Commission of Health, Youth Assembly, Istanbul Metropolitan Municipality.

\section{5 \\ Effect of Altitude on Fluoride Excretion and Retention in Children}

O. Sah*a , G. Atkinson ${ }^{\mathrm{a}}$, A. Maguire ${ }^{\mathrm{b}}$, F.V. Zohoori ${ }^{\mathrm{a}}$

o.sah@tees.ac.uk

aschool of Health and Social Care, Teesside University, Middlesbrough, UK; ${ }^{b}$ Centre for Oral Health Research, School of Dental Sciences, Newcastle University, Newcastle upon Tyne, UK

A greater prevalence of dental fluorosis has been reported in higher- versus lower-altitude communities. This may be due to specific changes in the body burden of fluoride which occurs in humans living at high altitude. This study aimed to quantify the effect of altitude on fractional urinary fluoride excretion (FUFE) and total daily fluoride retention (TDFR) in children living at lower $(<78 \mathrm{~m})$ and higher $(>1487 \mathrm{~m})$ altitudes in Nepal. Eighty-nine children, aged $4-5$ years completed the study: 42 living at lowerand 47 at higher altitude. Total daily fluoride intake (TDFI) was calculated from diet and toothpaste ingestion. Daily urinary fluoride (DUFE) excretion was measured by collecting a 24-hour urine sample and total daily fluoride excretion (TDFE) estimated as "DUFE + faecal fluoride excretion", assuming 10\% of TDFI was excreted through faeces. TDFR was estimated by subtracting TDFE from TDFI. FUFE was calculated as the ratio between DUFE and TDFI. A multivariable-adjusted general linear model was used to investigate differences in fluoride intake, excretion and retention between the 2 altitudes. TDFI was significantly greater at lower- compared with higher altitude (Mean \pm SD: $0.066 \pm 0.029 \mathrm{v}$ $0.045 \pm 0.020 \mathrm{mg} / \mathrm{kgbw} /$ day (95\% CI: +0.009 to $-0.030 \mathrm{mg} / \mathrm{kgbw} /$ day; $\mathrm{P}=0.001)$, and FUFE was significantly greater at lower- $\mathrm{v}$ higher altitude (Mean \pm SD: $54 \pm 19 \%$ v $46 \pm 16 \%$ (95\% CI: +0.626 to $+15.23 \%$; $\mathrm{P}=0.044)$. No statistically significant difference was observed for DUFE (adjusted to TDFI) or TDFR between lowerand higher altitude children. Effects of altitude on TDFR were small and non-significant. However, the statistically significant greater FUFE (\%) seen at lower altitude suggests greater body retention of fluoride at higher altitude which may increase the risk of dental fluorosis.

This study was supported by a grant from The Borrow Foundation. 


\section{Session 10 Clinical Studies}

\author{
116 \\ How Feasible Is Cross-Toothbrusing Implementation \\ Directed to Erupting Molars in Prescholar Children? \\ M.M. Braga*a,b, L.A. Pion a,c, B.A. Bernardino, \\ C.M. Castelo-Branco ${ }^{\mathrm{a}}$, D.P.Raggio ${ }^{\mathrm{a}}$, F.M.Mendes ${ }^{\mathrm{a}}$, J.C. Imparato ${ }^{\mathrm{a}, \mathrm{d}}$ \\ mmbraga@usp.br \\ aDental School, University of São Paulo, São Paulo, Brazil; \\ ${ }^{b}$ Centre for Health Economics and Policy Analysis (CHEPA), \\ McMaster Univeristy, Hamilton, Canada; ${ }^{\text {CRibeirao Preto Dental }}$ \\ School, University of Sao Paulo, Ribeirao Preto, SP, Brazil; dSao \\ Leopoldo Mandic, Campinas, SP, Brazil
}

This study investigated the operational and economic feasibility of introducing the cross-toothbrushing technique as a directed care to erupting 1st molars. This study was nested in a RCT focused on controlling initial caries lesions (SDFTrials-02NCT01508611). 194 children presenting erupting molar were randomly allocated to: anteroposterior vs. cross-toothbrushing. Children were trained to toothbrushing techniques by video demonstration and verbal instruction using a model. They were advised to use the techniques directed to their erupting molars and was assisted to brush after instructions. To evaluate the operational feasibility, children were watched toothbrushing after 6 and 12 months and their performance was checked (repetitions and position of toothbrush). We assumed that if the children failed in one of checked items, they would require reinforcement of toothbrusing instructions. The need for reorientation was compared between techniques using Poisson regression analysis and Relative Risks were calculated (RR; 95\% CI). To evaluate the economic feasibility, costs related to both techniques were considered, including the costs of reorientations during 12 months. The dentist was set as the provider of these instructions and Intention-To-Treat analyses used. 179 children were evaluated at 6-month and 170 at 12 -month follow-up (dropout: $6.7 \%$ and $11.4 \%$, respectively). Children took similar time to be orientated by both techniques (approximately $4 \mathrm{~min}, \mathrm{p}=0.73$ ). However, the cross-toothbrushing required twice more reinstruction than anteroposterior technique after 6 months $(\mathrm{RR}=1.96$; $1.53-2.50)$ and 12 months $(R R=1.52 ; 1.19-1.95)$. Less than 8 -year mother's schooling were associated with more need of reorientation $(\mathrm{RR}=0.80 ; 0.66-0.97)$. Costs of implementing a 12 -month cross-toothbrushing program were similar to the anteroposterior toothbrushing program (approximately, US\$15). Lower education did not impact on this parameter in any group $(\mathrm{p}=0.31 / 0.41)$. In conclusion, cross-toothbrushing is economically feasible to be indicated to 1st erupting molars. However, it may demand closer professional participation due to increased need of reorientation.

This study is the first to measure the operational impact of cross-toothbrushing compared to anteroposterior toothbrushing and to show it is an economic feasible option.

This project is funded by CNPq $(203632 / 2017-6 ; 309817 / 2015$ 3), CAPES, FAPESP (2012/50716-0, 2014/06925-9) and USP ProRectories.

\section{7 \\ Double-Blind, Placebo-Controlled Clinical Study to Asses the Efficacy of Streptotoccus dentisani in Caries Risk Reduction \\ M.D. Ferrer*a, A. Lopez-Lopez ${ }^{\mathrm{a}}$, C. Llena ${ }^{\mathrm{b}}$, T. Nicolescu ${ }^{\mathrm{b}}$, S. Perez-Vilaplana ${ }^{\text {b }}$ A. Boix-Garcia ${ }^{\mathrm{a}}$, M. Dzidic ${ }^{\mathrm{a}}$, A. Mira $^{\mathrm{a}}$ \\ ferrer_mde@gva.es \\ aFoundation for the promotion of Biomedical Research in the Valencian Community (FISABIO), Genomics and Health Area, Avda. Catalunya, 21. 46020 Valencia, Spain; ${ }^{b}$ Clínica Odontológica, Fundación Lluís Alcanys, Universitat de Valéncia, Valéncia, Spain}

Previous work from our laboratory has shown that $S$. dentisani was able to colonize the oral cavity for 2-4 weeks after application. Colonization was confirmed after detecting an increase between visits in the cfus of $S$. dentisani per ng of DNA measured in the supragingival plaque. The aim of the present work was to evaluate its clinical efficacy in reducing caries risk by a randomized, doubleblind, placebo-controlled parallel group study. Sixty volunteers enrolled in the study and were randomly assigned to a treatment or placebo group. S. dentisani colonization were not taken into ac- 
count for the recruitment. The treatment consisted of a buccoadhesive gel application $\left(2.5 \times 10^{9} \mathrm{cfu} /\right.$ dose $)$ with a dental splint during 5 minutes every 48 hours, for a period of 1 month (i.e. 14 doses). Dental plaque and unstimulated, drooling saliva samples were collected at baseline, 15 and 30 days after first application, and 15 days after the end of treatment. There were no significant differences between placebo and probiotic groups in any of the variables studied at baseline time. Stimulated salivary flow was significantly higher in the probiotic group at day 15 compared with the placebo ( 4.4 and $3.4 \mathrm{ml} / 5$ mins, respectively, $\mathrm{p}<0.05$, paired t-test). In the probiotic group, there was a $20 \%$ decrease in Plaque Index at day 30 (from 1.1 at baseline to 0.8 at day 30 ) which remained at day 45 but no significant differences were observed in the placebo group at any time point. The inter observer Kappa value for the plaque index was 0.85 . Only $64 \%$ of the participants in the probiotic group showed colonization of $S$. dentisani at day 30 . In the colonized patients, the salivary $\mathrm{pH}$ after a $10 \%$ sugar rinse was significantly higher in the probiotic group $(\mathrm{pH}=6.5)$ compared to the placebo $(\mathrm{pH}=6.1)$ at day $15(\mathrm{p}<0.05$, paired $\mathrm{t}-$ test). The difference in plaque $\mathrm{pH}$ after a $10 \%$ sugar exposure was also significantly lower in the probiotic group $(\mathrm{p}<0.05$, paired $\mathrm{t}$ test), which had on average 0.3 points lower decrease ( 0.5 in probiotic v.s 0.8 in placebo). In conclusion, the application of $S$. dentisani improved several oral health parameters associated with a lower dental caries risk, supporting its use as a probiotic to help in tooth decay prevention.

This work was funded by grantRTC-2015-4292-1 from the SpanishMinistry of Economy and Competitiveness.

\section{8 \\ Progression of Early Proximal Caries Lesions in Primary Molars After Resin Infiltration: A 12-Month Follow-Up RCT}

C.S. Sarti ${ }^{\mathrm{a}}$, C.C. Bonifacio ${ }^{\mathrm{b}}$, M.B. Vizzotto ${ }^{\mathrm{a}}$, J.A Rodrigues*a

jorodrigues@ufrgs.br

aSchool of Dentistry, Federal University of Rio Grande do Sul, Porto Alegre, Brazil; ' bepartment of Cariology Endodontology and Pedodontology, Academic Centre for Dentistry

Amsterdam (ACTA), Amsterdam, The Netherlands

The prevalence of proximal caries lesions in primary molars is high in many countries. This split-mouth controlled randomized clinical trial aimed to assess the progression of early proximal caries lesions on primary molars after resin infiltration. Twenty-eight children aged 3 to 8 years old took part of this study. Children had at least two proximal lesions radiographically extending to enamel or outer dentine. Digital bitewing radiographs were taken, visual plaque (VPI) and gingival bleeding indexes (GBI) were recorded and visual examination of caries was performed (baseline assessments). Proximal lesions were randomly allocated to two groups: resin infiltration (test) and no infiltration (control). Patients were classified as low (def-s $=0)$, moderate (def-s $=1-4)$ or high (def-s $>4$ ) caries experience. All children received 4-week topical fluoride application, dietary counseling, toothbrushing and flossing instructions. After a 12 -month period, three children were lost to follow up. The other 25 children had the baseline assessments re- peated, including digital radiographs. Digital images were compared analyzed by a blinded evaluator concerning lesion progression, an unweighted kappa value of 0.77 was found for intra-examiner reproducibility, and no statistical significant differences were observed between test and control groups (McNemar test, $\mathrm{p}=$ 0.45). Out of 25 lesions in the control and test groups, 19 and 15 had progressed, respectively. No significant relation between lesion progression and VPI, GBI and caries experience was observed (Mann Whitney Test, $\mathrm{p}>0.05$ ). This study showed no effect of infiltrating proximal caries lesions in primary molars after 12-month follow-up period.

\section{9 \\ Oral Colonization and pH Buffering by Streptococcus dentisani, A Probiotic Bacterium to Prevent Tooth Decay \\ A. López-López*a, M. Ferrera , C. Llena ${ }^{\mathrm{b}}$, T. Nicolescu $^{\mathrm{b}}$, A. Mira $^{\mathrm{a}}$ \\ mira_ale@gva.es \\ aGenomics and Health Area, FISABIO Foundation. Avda. Catalunya, Valencia, Spain; ${ }^{b}$ Dental clinic of the Lluís Alcanys \\ Foundation, University of Valencia, Valencia, Spain}

The bacterial species Streptococcus dentisani, isolated from dental plaque of caries-free individuals, has a double effect that has been proposed to potentially prevent tooth decay: an antimicrobial activity against oral pathogens and a $\mathrm{pH}$ buffering capacity through the arginolytic pathway. The aim of this work was to evaluate the colonization efficiency of $S$. dentisani and $\mathrm{pH}$ buffering in a pilot clinical study. Eleven adult volunteers with basal salivary $\mathrm{pH}$ lower than 7 were enrolled, in which the quantification of $S$. dentisani was determined for comparisons (baseline values). The probiotic was applied at a total dose of $10^{10} \mathrm{CFUs}$, administered in a buccoadhesive gel for 5 minutes, either in a single dose or daily for a week. It was also tested whether a professional cleaning improved colonization (split mouth model). Dental plaque and saliva samples were collected after 15 and 30 days of first application where amounts of $S$. dentisani and $\mathrm{pH}$ were measured by qPCR and reflectometry, respectively. As determined by Wilcox analyses, there was a significant increase in S. dentisani cells in supragingival dental plaque at day 15 from baseline but not at day 30 under both dosing schedules. Paired t-test analyses were applied to reveal differences in the $\mathrm{pH}$ values. Differences among cleaned and intact quadrants were not significant. There was a significant increase in basal salivary $\mathrm{pH}$ at day 14 (from 6.95 at baseline to 7.20 at day 14, $\mathrm{p}=0.046)$ in multidose patients, and in the $\mathrm{pH}$ after tooth brushing both at day $14(\mathrm{p}=0.026)$ and day $28(\mathrm{p}=0.027)$ also in multidose patients (from 7.0 at baseline to 7.19 and 7.39, at day 14 and 28 respectively). Differences among the two different dose regimes were not significant. The results indicate the S. dentisani is transiently able to colonize the oral cavity and to buffer oral $\mathrm{pH}$, especially after multiple dosing, suggesting a promising probiotic potential.

This work was funded by the Project RTC-2015-4292-1 of the Ministry of Economy and Competitiveness. 
120

\section{Glass lonomer Cement, Glass Carbomer and Compomer for ART Occlusoproximal Restorations: 3 Year RCT}

\author{
D.P. Raggio*a, I.C. Olegário a , D. Hesse ${ }^{\mathrm{b}}$, C.C. Bonifáciob, \\ F.M. Mendes ${ }^{\mathrm{a}}$
}

danielar@usp.br

aSchool of Dentistry, University of São Paulo, São Paulo, Brazil;

${ }^{b}$ ACTA, Amsterdam, The Netherlands

The aim was to evaluate the survival of Atraumatic Restorative Treatment (ART) restorations using high viscosity glass ionomer cement (GIC), compomer (COM) and glass carbomer (CAR) for occlusoproximal cavitated dentin caries lesions in primary molars. A total of 287 4-7 y old children were selected in Barueri, Brazil. The participants were randomly allocated in three groups: GIC, COM and CAR. All treatments were performed on school setting following ART premises. Evaluations were performed after 6, 12, 18,24 and 36 months by a single calibrated independent examiner using Roeleveld et al., 2006 criteria. Failure was considered if restoration needed repair, if it was missing or tooth showed signs/ symptoms of pulp involvement. Restoration survival was evaluated using Kaplan-Meier survival analysis and Log-rank test, while Cox regression analysis was used for testing association with clinical factors such as DMFT/dmft, jaw, side, occlusal contact, adjacent tooth $(\alpha=5 \%)$. The overall survival rate after 3 years of occlusal ART restorations was $51 \%(\mathrm{GIC}=58 \%$; $\mathrm{COM}=58 \%$; $\mathrm{CAR}=$ $38 \%)$. CAR restorations were less successful than GIC and COM $(\mathrm{p}<0.05)$. No difference was found between GIC and COM $(\mathrm{HR}=$ $0.88 ; \mathrm{CI}=0.56-1.37 ; \mathrm{p}=0.577)$. No association with clinical factors was observed. In conclusion, GIC and Compomer are clinically more successful than CAR in occlusoproximal ART restorations in primary molars.

Acknowledgement of sources of funding: FAPESP (2015/005653) and CNPq.

\section{1}

3-Year Effectiveness of ICCMS ${ }^{\mathrm{TM}}$ vs. Colombian NHS Caries-Management Schemes RCT in Schoolchildren

S. Martignon*a,b, A. Cortes ${ }^{\mathrm{a}}$, M.V. Usuga-Vacca ${ }^{\mathrm{a}}$, L.F. Gamboa a , M.C. Arango-De la Cruz', O.L. Cifuentes-Aguirred, N. Fortich-Mesa ${ }^{\mathrm{e}}$, K. Ramos-Martínez ${ }^{\mathrm{f}}$, J.A. Sanjuán-Acero ${ }^{\mathrm{g}}$, L. Alfaro ${ }^{\mathrm{a}, \mathrm{f}}$, L. Mejía ${ }^{\mathrm{a}, \mathrm{h}}$

martignonstefania@unbosque.edu.co

aUNICA - Caries Research Unit, Research Vice-rectory, Universidad El Bosque, Bogotá, Colombia; bental Innovation and Translation Centre, Kings College London, London, United Kingdom; ' Universidad del Valle, Cali, Colombia; ${ }^{\text {UUniversidad }}$ Autónoma de Manizales, Manizales, Colombia; ${ }^{\mathrm{e} C o r p o r a c i o ́ n}$ Universitaria Rafael Núñez, Cartagena, Colombia; fUniversidad de Cartagena, Cartagena, Colombia; 9 Universidad Colegio Odontológico Colombiano, Bogotá, Colombia; ${ }^{\text {hUniversidad }}$ Santo Tomas, Bucaramanga, Colombia

The aim of this study was to compare in a multicentre RCT setting the 3-year effectiveness of A) adjusted-ICCMS ${ }^{\mathrm{TM}}$ and B) Colombian-standard NHS caries-management schemes. After ethical approval and parent-informed consents, 240 7-year olds from 6 Colombian dental-school clinics were assessed by 6-trained examiners for: ICCMSTM caries-lesion likelihood (low/moderate/severe); visual plus radiographic caries lesions: ICDAS Initial (ICDAS-1/2) and Conventional dentine/cavitated Moderate/Extensive (ICDAS-3/6) plus fillings and missing teeth to account for the ICDAS caries experience (ICDAS-dmfs and ICDAS-DMFS), and caries activity assessment (active/inactive). The children were randomly allocated to management scheme (A or B) delivered by trained dentists.

Three-year follow-ups were conducted by 5-blind trained examiners on the remaining 187 subjects $(77.9 \%$; Scheme-A: $\mathrm{n}=92$; Scheme-B: $n=95)$. Baseline and 3-year prevalence of ICDAS-dmf and ICDAS-DMF combined was over $96 \%$. Overall mean of ICDAS-dmfs and ICDAS-DMFS combined was around 15 without significant differences between schemes and examination times.

From baseline respective Scheme-A and Scheme-B ICCMS ${ }^{\mathrm{TM}}$ caries-risk likelihood subjects' distribution (High: $\mathrm{n}=56, \mathrm{n}=52$; Moderate: $\mathrm{n}=30, \mathrm{n}=36$; Low: $\mathrm{n}=6, \mathrm{n}=7$ ), most decreased significantly from high/moderate likelihood with significant higher decrease in Scheme-A (Scheme-A: 73.3\%; Scheme-B: 64.7\%) (p < $0.05)$. In both groups there was a significant reduction in the mean number of active caries lesions (Initial/Moderate/Extensive) from baseline to 3-year follow-up (Scheme-A: $11.54 \pm 9.86$ to $5.17 \pm$ 4.51; Scheme-B: $12.02 \pm 9.56$ to $6.12 \pm 6.12)(\mathrm{p}<0.05)$, together with a consequent increase in the mean number of inactive caries lesions, without significant differences between schemes. Mean number of sealants increased significantly in both groups from baseline to 3 years (Scheme-A: $0.73 \pm 1.54$ to $1.90 \pm 2.37$; Scheme$B: 0.73 \pm 1.59$ to $2.75 \pm 2.74)$ with a significant higher figure in Scheme-B vs. Scheme-A $(\mathrm{p}<0.05)$. Finally, mean number of appointments was significantly higher in Scheme-A $(10.9 \pm 6.7)$ vs. Scheme-B $(10.0 \pm 5.3)(\mathrm{p}<0.05)$. Scheme-A showed a higher 3 -year caries-management effectiveness than Scheme-B in terms of caries-lesion likelihood decrease and less sealants application, but with more appointments. 
This study was partially funded by Universidad El Bosque, Kings College London, ACFF Colombian Chapter, Corporación Universitaria Rafael Núñez, Universidad Autónoma de Manizales, Universidad Colegio Odontológico Colombiano, Universidad de Cartagena, Universidad del Valle.

\section{2 \\ Atraumatic Versus Conventional Restorative Treatment for Root Caries Lesions in Older Patients: a Systematic Review \\ G. Göstemeyer*a , C. da Matab , G. McKenna ${ }^{\text {c }}$, F. Schwendicke ${ }^{\mathrm{a}}$ \\ gerd.goestemeyer@charite.de \\ aDepartment of Operative and Preventive Dentistry, Charité -

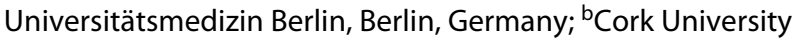 \\ Dental School and Hospital, University College Cork, Cork, Ireland; 'Centre for Public Health, Queens University Belfast, Belfast, UK}

Given the limited instrumental and material requirements, atraumatic restorative treatment (ART) might be advantageous over conventional restorative treatment $(\mathrm{CT})$ of root caries lesions in institutionalized older patients. The comparative clinical efficacy of both approaches and the robustness of underlying evidence is unclear. We aimed to systematically review this evidence using pairwise meta-analyis and trial sequential analysis (TSA). Randomized controlled trials reporting on survival of ART vs. CT for root caries restorations in older patients $\geq 60$ years) were included. Three electronic databases (PubMed, Embase, Cochrane CENTRAL) were systematically screened, and hand searches and crossreferencing performed. Trial selection, data extraction, and risk of bias assessment were conducted independently by two reviewers. Fixed- or random-effects per-protocol (PP), intention-to-treat (ITT) and scenario meta-analyses were performed, and TSA used to control for risk of random errors. From 235 studies identified, 4 trials (follow-up range 6-24 month) involving 233 patients (544 restorations) were included. All but one trial had high or moderate risk of bias. Risk of failure was borderline significantly increased for ART (OR [95\% CI] 1.72 [1.00/2.96]) in PP analysis but not in ITT analysis (1.32 [0.92/1.89]). Scenario analyses found great uncertainty introduced by attrition. According to TSA, no firm evidence was reached. There is insufficient data to clearly rule out whether differences between ART and CT for restoration of root caries lesions in older patients exist. Based on available data, ART might be associated with an increased risk of failure.

This study was funded by the authors and their institutions.

\section{3}

\section{Are Caesarean Section and ABO Group Related to Caries Severity? A Retrospective Cross-Sectional Study on Italian Children}

F. Cocco ${ }^{\mathrm{a}, \mathrm{b}}$, M. Arrica*a, M.G. Cagettia,c, P. Lingström ${ }^{\mathrm{b}, \mathrm{d}}$, S. Dessole ${ }^{\mathrm{e}}, \mathrm{G}$. Capobianco ${ }^{\mathrm{e}}, \mathrm{G}$. Campus ${ }^{\mathrm{a}, \mathrm{b}}$, Italian study group on ECC, M. Columbano, J. Davi, G. Frasconi, S. Nieddu, R. Pinna, L. Satta

mariantonietta.arrica@uniss.it aDepartment of Surgery, Microsurgery and Medical Sciences - School of Dentistry, University of Sassari, Sassari, Italy; bWHO Collaborating Centre of Milan for Epidemiology and Community Dentistry, University of Milan, Milan, Italy; 'Department of Biomedical, Surgical and Dental Sciences, University of Milan, Milan, Italy; ${ }^{\mathrm{d}}$ Department of Cariology, Institute of Odontology, The Sahlgrenska Academy, University of Gothenburg, Gothenburg, Sweden; eDepartment of Surgery, Microsurgery and Medical Sciences - Gynaecologic and Obstetric Clinic, University of Sassari, Sassari, Italy

The mode of delivery interplays with children's general health; moreover a connection among several diseases and different blood groups has been lately speculated. This preliminary survey aimed to investigate the potential association among mode of delivery, $\mathrm{AB} 0$ blood group and ECC in Italian toddlers. Data regarding the mode of delivery and blood groups were extracted from medical birth charts (2013-2014) of 6775 toddlers (24-30 months) living in Sardinia, Italy. Power analysis was performed (3.1.9.2-Apple $\mathrm{G}^{*}$ Power $($ ) adding a prevalence ratio of $40 \%$ to safeguard against the risk of disease spread. Error probability of 0.05 and actual power of 0.95 were considered. The total sample size was set to 236 . Invitations reached 2191 toddlers and 244 were eventually examined. Prior calibrated dentists from the Italian study-group on ECC $(\mathrm{K}=0.85-0.91)$ recorded the International Caries Detection and Assessment System (ICDAS) scores. Caries severity (CS) was calculated as the summation of ICDAS multiplied by the number of lesions [ $\Sigma(0-6)$ ICDAS(score)*n(lesions)]. The Mann-Whitney $\mathrm{U}$ test was applied to between-group comparisons. $47.80 \%$ ( $\mathrm{n}=$ 117) of children was delivered via cesarean section, while $52.20 \%$ $(\mathrm{n}=127)$ was vaginally delivered. CS was higher in children delivered by $\mathrm{C}$-section $(3.66 \pm 6.59)$ compared to the ones vaginally delivered $(\mathrm{CS}=3.03 \pm 5.52 ; \mathrm{p}>0.05)$. CS was statistically significantly lower $(\mathrm{p}<0.02)$ in toddlers with $\mathrm{AB}$ blood group $(\mathrm{CS}=$ $1.86 \pm 2.34)$, followed by toddlers with A blood group $(C S=2.29 \pm$ $4.41)$ and toddlers with 0 blood group $(\mathrm{CS}=2.75 \pm 5.34)$. B blood group showed the highest caries severity $(C S=3.60 \pm 6.24)$. In conclusion a possible bias related to the high CS frequency in our sample might contribute to reject our hypotheses. A higher CS level was observed in toddlers with B blood group.

All authors declare no conflict of interest and no financial support to this study. 


\section{4}

\section{Trial Sequential Analysis on Sealing or Infiltration of Proximal Carious Lesions}

Falk Schwendicke*, Gerd Göstemeyer, Seif Reda, Joachim Krois

falk.schwendicke@charite.de

Department of Operative and Preventive Dentistry, Charité Universitätsmedizin Berlin, Berlin, Germany

Micro-invasive (sealing, infiltration) treatment seems more efficacious for arresting early (non-cavitated) carious lesions than non-invasive treatment (NI). Uncertainty remains as to robustness of the underlying evidence. We aimed to perform trial sequential analysis (TSA) on data obtained from a systematic review of this evidence. Three electronic databases (Medline, Embase, Cochrane Central) had been searched, complemented by hand searches and cross-referencing. Randomized controlled trials comparing micro-invasive strategies against each other, NI or placebo for managing early (non-cavitated) proximal carious lesions had been included. The primary outcome was radiographically assessed lesion progression. After accounting for clustering (ignoring split-mouth effects), TSA employing a random-effects DL-model was applied. For TSA, a type I error risk of $\alpha=0.05$ and a type II error risk of $\beta=0.20$ were assumed. A relative risk reduction (RRR) based on interventional effect of $20 \%$ was defined as worthwhile. The required information size (RIS) was adjusted for diversity (DARIS). Firm evidence was assumed to be reached when the Z-curve crossed the TSMB for benefit or harm before the DARIS was reached. Thirteen split-mouth studies (486 participants), some of them additionally clustered, were included. Mean follow-up was 25 months ( $\min / \max 12 / 36$ months). Firm evidence on the superior efficacy of sealing/infiltration over NI was reached (OR; $95 \%$ CI: $0.24 ; 0.18-0.32,1236$ lesions) with the Z-curve crossing the TSMB for benefit before the DARIS (970 lesions) was reached. Firm evidence was also reached on the superior efficacy of infiltration (OR; 95\% CI: 0.29; 0.18-0.46, 728 lesions) and sealing (OR; 95\% CI: 0.22; 0.15-0.33, 508 lesions) over NI. There is robust evidence that sealing and infiltration are more efficacious than NI for arresting proximal carious lesions.

This study was funded by the authors and their institutions.

\section{5 \\ Music May Improve Toothbrushing in Teens \\ Mohammad Alkilzy*, Beatrice Formann, Christian Splieth \\ alkilzym@uni-greifswald.de \\ Department of Preventive and Pediatric Dentistry/University of Greifswald, Greifswald, Germany}

Performing proper toothbrushing is a complicated process for children and adolescences to become competent in which possibly could be improved with motivation by music. The aim was to investigate the effect of music for improving toothbrushing in early teenagers. In this prospective, controlled, singleblinded, randomized clinical trial, 35 children between 11-13 years old (mean age $12.0 \pm 0.9$ years) were randomly assigned to the test $(n=18)$ and control group $(n=17)$. All children were provided with manual toothbrushes, fluoridated toothpaste and oral health instructions including two min. toothbrushing twice a day. Only the children of the test group were instructed to brush their teeth in combination with two minutes of music. At baseline and recall after three months, plaque and gingival indices (QHI, PBI) were recorded and transferred to WinStat 3.1. KolmogorovSmirnov-Test showed normal distribution of the values and, therefore, $\mathrm{t}$-tests and $\mathrm{X}^{2}$-test were used for analysing data for the two groups. At baseline, there were no significant differences between test and control group regarding plaque and gingival indices (QHI: $3.18 \pm 0.53$ and $3.41 \pm 0.76$, resp., $\mathrm{p}=0.94$; PBI: $0.45 \pm$ 0.18 and $0.58 \pm 0.22$, resp., $\mathrm{p}=0.59$ ). At the 3 -month recall, test and control groups showed statistically significant improvement in oral health indices to baseline: QHI $2.33 \pm 0.71$ and $2.82 \pm 1.02$ resp., $\mathrm{p}<0.01$, the probability of error was in test group lower than control $(\mathrm{p}=0.000087$ vs. $\mathrm{p}=0.00104)$. PBI $0.36 \pm 0.15$ and $0.50 \pm 0.24$, resp., $\mathrm{p}<0.01$, the reduction was highly significant $(\mathrm{p}=0.00092)$ in the test group, but less significant $(\mathrm{p}=0.037)$ in the control. In conclusion, music may present a successful motivation tool for improving toothbrushing in children and adolescences.

\section{6 \\ Effect of a Daily Dose of Snacks Containing Stevia Rebaudiana or Maltitol in High Caries Risk School Children}

F. Cocco ${ }^{\mathrm{a}, \mathrm{b}}$, M.G. Cagetti ${ }^{\mathrm{b}, \mathrm{c}}$, R. Livesu ${ }^{\mathrm{a}}$, N. Camoni ${ }^{\mathrm{c}}$, P. Lingström ${ }^{\mathrm{b}, \mathrm{d}}$, G. Campus*a,b

gcampus@uniss.it

aDepartment of Surgery, Microsurgery and Medical Sciences, School of Dentistry, University of Sassari, Viale San Pietro 43/C, I-07100 Sassari, Italy; ${ }^{b}$ WHO Collaborating Centre of Milan for Epidemiology and Community Dentistry, University of Milan, Milan, Italy; 'Department of Biomedical, Surgical and Dental Sciences, University of Milan, Milan, Italy; ${ }^{\text {d Department of }}$ Cariology, Institute of Odontology, Sahlgrenska Academy, University of Gothenburg, Gothenburg, Sweden

A randomised clinical pilot study was designed to evaluate the capacity of sugar-free snacks to modify plaque $\mathrm{pH}$ and salivary mutans streptococci (ms) and Lactobacilli ( $\mathrm{Lb})$ concentration in a group of schoolchildren who received products containing nosugar or sugary snacks for 6 weeks. A total of 121 subjects $(38.5 \%$ boys $/ 61.5 \%$ girls, age range $6-8$ yy; $1-4$ dentine lesions and a $\mathrm{ms}$ concentration of $\geq 10^{5} \mathrm{CFU} / \mathrm{mL}$ ) were randomly assigned to three groups: stevia group, maltitol group and sugar group. Children were instructed to consume the snacks twice a day, one in the morning and one in the afternoon, with other dietary modifications. Ms and Lb counts in saliva were assessed using the dip-slide technique (CTR bacteria, Ivoclar Vivadent, Germany) and interproximal plaque $\mathrm{pH}$ evaluated using $\mathrm{pH}$ indicator strips $(\mathrm{pH}$ range 4.0-7.0; Merck, Germany), following a 10\% sucrose-rinse. The area under the curve between reference $\mathrm{pH}$ line and the $\mathrm{pH}$ curve (AUC5.7 and AUC6.2) was calculated. Children were eval- 
uated at baseline, at the end of the administration ( 6 weeks) and re-evaluated after 18 weeks. The area under the curve at pH 5.7 was statistically significantly lower $(\mathrm{p}=0.01)$ in the stevia group, varying from $10.5 \pm 0.4$ at baseline to $9.2 \pm 0.3$ after the experimental period. The comparison of AUC5.7 values recorded in the three groups was also statistically significantly different at 6 weeks ( $p=0.04)$. In stevia and maltitol groups, ms and Lb concentrations decreased, but the reduction was statistically significant different only in the stevia group $(p<0.01)$. In conclusion the main result of the study is that the use of a stevia-based snack improves the oral health status by both controlling the plaque acidogenicity and reducing the number of caries-associated bacteria.

The study was sponsored by Galbusera SpA Italy.

\section{7 \\ Efficacy of Propolis Toothpaste Against Streptococcus mutans in Orthodontic Patients: A Randomized and Double-Blind Study}

\author{
M.A.L. Lotif*a, L.A.R. Valadasa, J.H.C. Furtado Jra , F.O. Chagasa, \\ I.S. Arrudab , S.G.C. Fonseca a , F.V. Fechine, , M.H.R. de Aguiar ${ }^{\text {b }}$, \\ T.C. Simoes ${ }^{\mathrm{b}}$, J.F. Mendonça ${ }^{\mathrm{b}}$, G.L. Bezerra ${ }^{\mathrm{d}}$, P.L.D. Lobo ${ }^{\mathrm{b}}$, \\ M.M.F. Fonteles ${ }^{\mathrm{a}, \mathrm{c}}$ \\ mara_lotif@hotmail.com \\ aPharmacy, Dentistry and Nursery College, Federal University \\ of Ceara, Fortaleza, Brazil; ${ }^{b}$ Department of Dentistry, Federal \\ University of Ceara, Fortaleza, Brazil; ' Department of \\ Pharmacology, Federal University of Ceara, Fortaleza, Brazil; \\ dPaulo Picanço College, Fortaleza, Brazil
}

The objective of this study was to evaluate the clinical efficacy of a fluoridated toothpaste (1500 ppm) containing 1\% Brazilian Red Propolis (BRP) extract against salivary Streptococcus mutans in orthodontic patients. Experimental approach: This was a randomized and double-blind study. Forty participants, caries-free (ICDAS 0), aged 12-18 years, from both genders were selected and randomized into two groups. The first group received BRP toothpaste and the second group received fluoridated common toothpaste. The participants brushed their teeth for $2 \mathrm{~min}$ thrice a day for 30 days. Saliva stimulated was then collected before starting treatment (D0), 30 days after day 0 (D30) and 90 days after day 0 (D90) to verify the effectiveness of BRP toothpaste treatment in the reduction of $S$. mutans. The microbiological analysis was repeated twice, establishing dilutions of 1:100 mL and 1:1000 mL on Mitis salivarius-bacitracin agar (MSB). S. mutans isolates were identified by its characteristic colony morphology (dark blue, small, irregularly margined and adherent) and the values were expressed as $\log _{10}(\mathrm{CFU} / \mathrm{mL})$. Statistics were carried out by applying Friedman and Mann-Whitney tests. We found the $S$. mutans levels were as follows on the first dilution: BRP Group [D0: 14.68 (23.96); D30: 4.87 (9.63); D90: $2.55(4.42)^{*}$; Common toothpaste [D0: 5.68 (12.10); D30: 2.71 (5.29); D90: $\left.18.54(23.47)^{*}\right]$. On the second dilution: BRP Group [D0: 12.29 (26.03); D30: 3.94 (7.85); D90: 1.76 $(2.98)^{*}$ ]; Common toothpaste [D0: 3.34 (4.69); D30: 2.01 (2.51); D90: 8.96 (12.69)]. BRP Group demonstrated a significant $S$. $m u$ tans reduction on the first dilution: D90 versus D0 $(\mathrm{p}=0.0052)$, when compared to common toothpaste group. In conclusion, these data indicate that the BRP toothpaste showed antimicrobial activity against $S$. mutans for up 90 days in orthodontic patients.
128
Clinical Efficacy of a $1 \%$ Brazilian Red Propolis Toothpaste for Gingivitis Control in Orthodontic Patients
L.A.R. Valadas*a, J.H.C. Furtado Jra , F.O. Chagas ${ }^{\mathrm{a}}$, I.S. Arruda ${ }^{\mathrm{b}}$, S.G.C. Fonseca ${ }^{\mathrm{a}}$, F.V. Fechine ${ }^{\mathrm{c}}$, M.H.R. de Aguiar ${ }^{\mathrm{b}}$, T.C. Simoes ${ }^{\mathrm{b}}$, J.F. Mendonça ${ }^{\mathrm{b}}$, G.L. Bezerra ${ }^{\text {d }}$, P.L.D. Lobo ${ }^{\mathrm{b}}$, M.M.F. Fonteles ${ }^{\mathrm{a}, \mathrm{c}}$
lidiavaladas@gmail.com
aPharmacy, Dentistry and Nursery College, Federal University

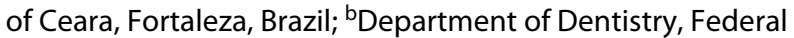 University of Ceara, Fortaleza, Brazil; ' Department of Pharmacology, Federal University of Ceara, Fortaleza, Brazil; dPaulo Picanço College, Fortaleza, Brazil

The objective of this study was to evaluate the clinical efficacy of a fluoridated toothpaste (1500 ppm) containing 1\% Brazilian Red Propolis (BRP) extract for gingivitis in orthodontic patients. This was a randomized and double-blind study. 100 participants, caries-free (ICDAS 0, kappa $=0.91$ ), aged $12-18$ years, from both genders were recruited in public schools, selected after clinical examination performed by two calibrated examiners and randomized into two groups. The first group (G1) received BRP toothpaste and the second group (G2) received a fluoridated common toothpaste. The participants brushed their teeth for 2 min thrice a day for 30 days. Data on gingival bleeding index (GBI) were recorded on days $0($ kappa $=0.88)$ and $30($ kappa $=0.94)$ to verify the effectiveness of the G1 toothpaste treatment in the GBI reduction. Comparisons between different times were carried out by Wilcoxon test and Tukey's multiple comparisons test. The GBI (mean \pm standard deviation) were as follows: G1 (D0: $50.58 \pm 16.37$; D30: $28.34 \pm$ 14.05); G2 (D0: $43.75 \pm 16.95$; D30: $39.43 \pm 16.60)$. As compared with common toothpaste, GBI significantly decreased in the BRP group $(\mathrm{p}<0.05)$. There were no reported side effects. In conclusion, these data indicate that the BRP toothpaste decreased the GBI for up 30 days in orthodontic patients presenting gingivitis. 


\section{9}

Enhancing Fluoride Varnish Efficacy and Compliance by Means of Calcium-Containing Gummy Bears

F. Lippert*a, L. Al Dehailan ${ }^{\text {a }, ~ G . A . ~ C a s t i b l a n c o ~}{ }^{\text {a }}$, A. Tagelsira, C. Buckley ${ }^{\mathrm{a}}$, G.J. Eckert ${ }^{\mathrm{b}}$

flippert@iu.edu

aDepartment of Cariology, Operative Dentistry and Dental

Public Health, Indiana University School of Dentistry, Indianapolis, Indiana, USA; ${ }^{\circ}$ Department of Biostatistics, Indiana University School of Medicine, Indianapolis, Indiana, USA

This in vivo study determined whether consumption of calcium-containing gummies prior to fluoride varnish application enhances fluoride retention in plaque and compliance with post-varnish application instructions. The present study followed a multicenter, parallel, randomized, and laboratory analyst-blind design. Following IRB approval, parent consent and child assent, 44 subjects (7-12 years) were enrolled. Subjects were randomized to either a gummy or no-gummy study group. A baseline plaque sample was obtained after a wash-out period. Fluoride varnish (CavityShield, 3M ESPE) was applied to all teeth in both groups; subjects in the gummy group received two calcium-containing gummies (Gummy Cuties Calcium, Natural Dynamix) prior to varnish application. Subjects were given two questionnaires to complete (subject and parent) to investigate adherence to post-treatment instructions. Three days after varnish application, a second plaque sample was obtained. Plaque samples were analyzed for plaque fluid and solid fluoride concentrations. Fluoride data were analyzed using Wilcoxon Rank Sum tests, questionnaire data using Pearson chi-square tests. Plaque fluid fluoride did not change significantly pre- to post-treatment in the gummy group (mean \pm sd: $8.8 \pm$ $5.7 \mu \mathrm{mol} / \mathrm{l}$ vs. $10.0 \pm 6.3 \mu \mathrm{mol} / \mathrm{l} ; \mathrm{p}=0.265$ ) or in the no-gummy group $(8.1 \pm 4.4 \mu \mathrm{mol} / \mathrm{l}$ vs. $16.1 \pm 20.0 \mu \mathrm{mol} / \mathrm{l} ; \mathrm{p}=0.058)$. The two groups were not significantly different for plaque fluid fluoride pre-treatment $(\mathrm{p}=1.000)$, post-treatment $(\mathrm{p}=0.904)$, or change $(\mathrm{p}=0.904)$. Plaque solid fluoride did not change significantly preto post-treatment in the gummy group $(0.89 \pm 1.10 \mu \mathrm{mol} / \mathrm{g}$ vs. $1.37 \pm 1.77 \mu \mathrm{mol} / \mathrm{g} ; \mathrm{p}=0.073)$ or in the no-gummy group $(0.68 \pm$ $0.77 \mu \mathrm{mol} / \mathrm{g}$ vs. $2.01 \pm 5.00 \mu \mathrm{mol} / \mathrm{g} ; \mathrm{p}=0.190$ ). The two groups were not significantly different for plaque solid fluoride pre-treatment $(\mathrm{p}=1.000)$, post-treatment $(\mathrm{p}=0.466)$, or change $(\mathrm{p}=0.874)$. No significant differences were found between groups for questionnaire responses. Adherence to post-treatment instructions was good. The present study failed to demonstrate an effect of calciumcontaining gummies in enhancing plaque fluoride retention.

This work was funded by a faculty research grant from the Delta DentalFoundation.

\section{0 \\ Standardized Caries Risk Assessment Methods: A Comparative Pilot Study}

\author{
M.G. Cagetti*a,b , G. Bontàa, ${ }^{\mathrm{a}, \mathrm{b}}$, F. Cocco $^{\mathrm{b}, \mathrm{c}}$, P. Lingström ${ }^{\mathrm{b}, \mathrm{d}}$, \\ L. Strohmenger ${ }^{\mathrm{a}, \mathrm{b}}$, G. Campus ${ }^{\mathrm{b}, \mathrm{c}}$ \\ maria.cagetti@unimi.it \\ aDepartment of Biomedical, Surgical and Dental Sciences, \\ University of Milan, Milan, Italy; ${ }^{b}$ WHO Collaborating Centre of \\ Milan for Epidemiology and Community Dentistry, University \\ of Milan, Milan, Italy; ' Department of Surgery, Microsurgery \\ and Medical Sciences - School of Dentistry, University of

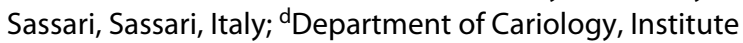 \\ of Odontology, The Sahlgrenska Academy, University of \\ Gothenburg, Gothenburg, Sweden
}

This pilot study aims to analyse the different caries risk levels measured using three standardized models of caries risk assessment, Cariogram, CaMBRA and Previser. A sample size calculation for pilot study was performed following Viechtbauer et al. (2015), with a minimum 65 subjects required. One hundred subjects attending the Dental School of the University of Milan (mean age $23 \pm 3.11 \mathrm{yy}$ ), were invited to participate. Sixty-eight subjects were enrolled and interviewed to record general health, oral hygiene practices, dietary habits and fluoride exposure. Caries experience (DMFT index) and amount of plaque (Silness and Loe plaque index) were measured. Salivary flow rate, salivary buffer capacity and salivary concentration of caries-associated bacteria, mutans streptococci and lactobacilli, were also recorded. Finally, for each subject the risk level was calculated using CaMBRA, PreViser and Cariogram models. Concordance correlation agreement (CCA) was calculated to determine how far the observed data deviate from the line of perfect concordance. The Bradley-Blackwood test $(\mathrm{BBt})$ was used for a simultaneous test of their means and variances. Data show a range of agreement from low to good between the risk level calculated using the three models. CCA was 0.37 (95\% CI 0.21-0.53) with a BBt $=21.22(\mathrm{p}<0.01)$ between Cariogram and Cambra, CCA $=0.43(95 \%$ CI 0.26-0.60) with a BBt $=$ 13.11 ( $\mathrm{p}<0.01$ ) between Cariogram and Previser, and finally $\mathrm{CCA}=0.65(95 \%$ CI $0.52-0.78)$ with a $\mathrm{BBt}=8.97(\mathrm{p}<0.01)$ between Cambra and Previser. Cariogram, CaMBRA and Previser do not produce concordant results. The findings of this pilot study underline how the calculation of the risk level even using standardized models is a very difficult goal not yet fully achieved. 
131

\section{Changes of Bisphenol-A Concentrations in Urine According to the Sealant Filling on Children}

T.J. Park ${ }^{* a}$, Y.H. Choi ${ }^{\mathrm{a}, \mathrm{b}}$

cyh1001@knu.ac.kr

aDepartment of Preventive Dentistry, School of Dentistry, Kyungpook National University, Daegu, Republic of Korea; ${ }^{b}$ Department of Preventive Dentistry, Osaka University Graduate School of Dentistry Suita, Osaka, Japan

The objectives of this study were to investigate the changes of Bisphenol-A (BPA) concentrations in urine before and after the sealant filling, and the relation of BPA and number of sealed tooth surfaces. Thirty-one children aged 6 and 7 years old who had no sealants or resin fillings from three elementary schools in Daegu city were selected. Urine sample were collected before and after sealant placement for up to 24 hours. Care givers gave informed consent. All urine samples were analyzed for the BPA and creatinine levels at Seegene Medical. Statistical analysis was performed using Friedman test, Scheirer-Ray-Hare test and Repeated measures generalized linear mixed model using SPSS version 22.0. The changes of BPA concentrations [expressed per $g$ of creatinine in order to standardize BPA concentrations between samples] were $3.49 \pm 3.11 \mu \mathrm{g} / \mathrm{g}$ creatinine before and increased to $4.91 \pm 4.09 \mu \mathrm{g} / \mathrm{g}$ creatinine after $2 \sim 3$ hours and $4.15 \pm 3.02 \mu \mathrm{g} / \mathrm{g}$ creatinine after 24 hours. The more sealed tooth surfaces, the higher BPA concentration in children, but this did not reach statistical significance $(\mathrm{p}>$ 0.05 ). In conclusion the concentrations of BPA were highest at 2-3 hours after sealant filling and decreased at 24 hours. Exposure to sealants contributes to BPA in urine of children.

This research was supported by Basic Science Research Program through the National Research Foundation of Korea funded by the Ministry of Education (NRF-2014025412).

\section{2 \\ Magnetic Resonance Characterization of the Radiated Parotid Gland Tissue Structure Using T2 Mapping Technique}

K. Cankar*a, J. Vidmar ${ }^{\mathrm{a}, \mathrm{c}}$, M. Groselj ${ }^{\mathrm{b}}$, I. Sersac , Z. Finderle ${ }^{\mathrm{a}}$

ksenija.cankar@mf.uni-lj.si

anstitute of Physiology, Faculty of Medicine, University of

Ljubljana, Ljubljana, Slovenia; 'bepartment of Dental Diseases, University Medical Centre Ljubljana, Ljubljana, Slovenia; ' Jozef Stefan Institute, Laboratory for Magnetic Resonance Imaging, Ljubljana, Slovenia

Radiation therapy with doses above 40 Gy irreversibly damages salivary glands, causing their atrophy and necrosis with hypofunction. Due to its high sensitivity and optimal tissue contrast, advanced magnetic resonance (MR) quantification techniques, such as T2 mapping, could detect post-radiation ultrastructural tissue changes in salivary glands. The aim of the present study was to assess ultrastructural tissue post-radiation changes in parotid glands in patients with head and neck tumours using advanced MR technic T2 mapping. Seven patients (age $56.8 \pm 11.1$ years) with head and neck tumours who had undergone radiation therapy with doses between 60 and 70 Gy were included in the study. MR imaging was performed at least 7 months after the end of the radiation therapy on 3T MRI System (Achieva, Philips, Netherlands) with dedicated imaging protocol which included multi-echo spinecho T2-weighted sequence (TR/TE ms/echo time; 2000/8, 16, 24, 32,40 and $47 \mathrm{~ms}$ ). T2 relaxation times were obtained from reconstructed T2 maps by using a pixel-wise; mono-exponential nonnegative least-squares fit analysis (ImageJ, NIH). All examinations were performed bilaterally on both parotid glands. Healthy volunteers with normal salivary function served as controls. In patients with head and neck tumours after radiation therapy, parotid glands T2 relaxation times $(113.8 \mathrm{~ms} \pm 20.7)$ were significantly higher compared to values obtained in parotid glands of healthy controls $(87.6 \mathrm{~ms} \pm 10.1)$ (t-test, $\mathrm{p}=0.001)$. In conclusion advanced MR technic T2 mapping is a highly feasible and reproducible technique, which can detect and even quantify tissue changes in salivary glands after the radiation therapy.

Funded by the Slovenian research agency project J3-7518.
133

\section{Calibration in Radiographical Diagnostics and Removal of Carious Tissue in Deep Lesions}
Azam Bakhshandeh*a, Mette Borum ${ }^{\mathrm{b}}$, Lars Høvenhoff, Hanne Gaard ', Helena Fransson ${ }^{\mathrm{e}}$, Lina Stangvaltaitef, Lars Bjørndalg
azamba@sund.ku.dk
aDepartment of Odontology, University of Copenhagen, Copenhagen, Denmark; 'bPublic Dental Health Care Service, Aarhus Municipality, Aarhus, Denmark; 'Public Dental Health Care Service, Randers Municipality, Høje-Taastrup, Denmark; ${ }^{d}$ Public Dental Health Care Service, Copenhagen

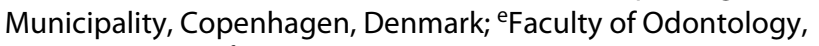 Malmö, Sweden; fDepartment of Clinical Dentistry, UiT The Arctic University of Norway, Oslo, Norway; '9Department of Odontology, University of Copenhagen, Copenhagen, Denmark

Sufficient evidence is lacking in literature supporting whether a 1 stage selective removal is better or worse compared to a 2 stage approach in permanent teeth with primary deep carious lesion. One objective for improving is to investigate the beneficial and harmful effects of selective carious removal versus stepwise removal by applying a randomized clinical trial (RCT). Prior to the initiation of a RCT we aimed to evaluate the investigator agreement in A) inclusion of deep lesions defined as being into the pulpal quarter of the dentine, and B) selective removal at central lesions sites following first stage. Case-based PowerPoint presentations were prepared including 25 radiographical and 13 clinical cases. The radiographical scorings were: not deep enough, deep enough, too deep. The clinical choices were: too little, optimal, too much carious removal. The radiographs were scored twice with 3 hours in between scorings while the clinical cases were scored once. Digital voting system (Clickers) was used for anonymous registration. 
52 dentists from 6 municipalities in Denmark and 2 dentists from Lithuania were included. Two of the authors $(A B+L B)$ scored the cases which were used as 'golden standard' for statistical analyses. Regarding the radiographical agreements the intra-examiner kappa for the 54 dentists varied from poor to good agreement $(\mathrm{k}=$ $0-0.7)$. Inter-examiner kappa, calculated between the 'golden standard' and the 54 dentists, varied from poor to almost perfect agreement $(\mathrm{k}=0.1-0.9)$. Regarding clinical agreements the inter-examiner kappa, calculated between the 'golden standard' and the 54 dentists, varied from poor to almost perfect agreement $(\mathrm{k}=0-0.8)$. In conclusion, variations in inter- and intra-examiner kappa-values were found indicating that repeating monitoring centre visits are mandatory for the reporducible performance of carious removal multicentre trials.

\section{4 \\ Cambodia Smile - Caries Prevention by Primary Health Care Providers}

\author{
B. Turton*a, C. Durward ${ }^{\text {b }}$, S. Sopharith ${ }^{\text {b }}$, F. Crombie ${ }^{\text {a }}$, \\ D.J. Manton ${ }^{\text {a }}$
}

Bethy.turton@gmail.com

${ }^{a}$ Oral Health Cooperative Research Centre, Melbourne Dental School, University of Melbourne, Melbourne, VIC Australia;

bental Department, University of Puthisastra, Phnom Penh, Cambodia

Young children in Cambodia suffer from a severe burden of carious lesions which are initiated early in life. The value of Fluoride Varnish (FV) interventions for Early Childhood Caries (ECC) has been questioned recently; however, evidence suggests that prevention in the early years of life can have long-term benefits. 262 Mother-child dyads were recruited at birth and followed for two years (intervention group) and a contemporaneous sample of 184 participants from ages 18 to 30 months were recruited from surrounding districts (control group). Intervention group participants had Six opportunities for oral health information (OHI), toothbrushes and fluoride toothpaste, fluoride varnish (NFlor protect; Ivoclar Vivadent), and dietary advice integrated with existing vaccinations, de-worming, and vitamin A schedule. At follow-up, intraoral examinations were conducted to detect carious lesions including white spot lesions using the South East Asian recommendations for epidemiological surveys of ECC. 185/262 (70.2\%) of the intervention group were followed-up and there was no significant difference in age (in months) between the control group $(\mathrm{N}=184 ; 22.2 \pm 2.4)$ and the intervention group $(21.9 \pm 2.6)$. There was no difference in caries experience by sex. A significant ( $p$ $<0.001$ ) difference existed in the number and prevalence of carious lesions between the intervention group $(2.9 \pm 3.0$ and $68.1 \%)$ and control group $(8.7 \pm 4.9$ and $94.0 \%)$ respectively. When cavitated lesions (alone) were considered, those children who had only $\mathrm{OHI}$ during the first 6 -months of life had a $25.0 \%$ preventive increment while those who received FV in addition had a $62.5 \%$ preventive increment when compared to the control group. In conclusion FV in combination with $\mathrm{OHI}$ was more effective at preventing cavitated lesions than $\mathrm{OHI}$ alone.

\section{5 \\ Selective Carious Removal in Medium and Deep Occlusal Cavitated Lesions with Preoperative defined Lesion Activity - Preliminary results}

E. Garcia ${ }^{a}$, L. Bjørndal ${ }^{\text {b }}$, S.C. Leal*a

sorayaodt@yahoo.com

aDepartment of Dentistry, Faculty of Health Science, University of Brasília, Brasília, Brazil; b Department of Odontology,

University of Copenhagen, Copenhagen, Denmark

Will carious lesion activity matter in cavitated lesions of different depths following selective carious removal? The aim of this clinical trial was to test if the lesion activity in deep and medium sized occlusal carious lesions in primary molars influence the clinical outcome, following selective carious removal. A power calculation showed that 60 teeth were needed. Forty children aged 3-6 yrs-old presenting cavitated occlusal carious lesions in primary molars were included. The depth of the le-sions was assessed by an independent examiner through bitewing X-rays. One calibrated examiner using a pre-tested lesion activity scoring system $(\mathrm{k}=$ $0.90)$ assessed cavities preoperative status. Treatments were performed using selective carious removal to soft or firm dentine, to the extent a restora-tion could be placed. Cavities were restored using high-viscosity glass ionomer (Equia Forte, GC America). Optimal restoration and absence of pulp complications at 6 months follow-up were con-sidered a successful outcome. Restorations and pulp vitality were assessed by an independent exam-iner blinded to the cavity depth/carious activity $(\mathrm{k}=1)$. Preliminary results on the basis of the assess-ment of 20 cavities ( 8 deep/12 medium) of which 15 were of slow carious progression and 5 highly carious active showed that all teeth could be treated without pulp complications, independently of the lesion depth/activity. After 6 months, 1 failure was observed (fistula) in a deep cavity that received the maximum score for carious activity. A long follow-up period is needed to determine whether the activity of cavitated dentine lesions is a factor that influence on restoration and tooth vitality follow-ing selective carious removal.

This study was partially supported by National Council for the Scientific and Technological Development from the Brazilian Government - CNPq Grant 306852/2016. 


\section{Session 11 Diagnostics: Clinical Studies Pulp-Dentinal Reactions}

\author{
136 \\ Initial Caries Lesion Registered During Operative \\ Treatment on the Neighboring Tooth: What Would \\ You Do? \\ A. Cartagena*a , A. Bakshandeh ${ }^{\mathrm{b}}$, I. Floriano ${ }^{\mathrm{c}}$, K.R. Ekstrand ${ }^{\mathrm{b}}$ \\ acartagenag@gmail.com \\ aDepartment of Pulpal Biology and Endodonthics, \\ Dental School at Finnis Terrae University, Santiago, Chile; \\ ${ }^{b}$ Department of Cariology and Endodonthics, Dental School \\ at Copenhague University, Copenhagen, Denmark; ' Dentistry \\ Course, Uninovafapi University Centre, Piauí, Brazil
}

The aim was to determine what is the treatment choice for a white spot lesion (WSL) corresponding to an ICDAS 2-lesion clinically found on an approximal surface of a posterior tooth, during operative treatment on the approximal surface of neighboring tooth. A simple case-based questionnaire was performed and sent by email to a group of teachers at the Finis Terrae University in Santiago, Chile. The file was a digital presentation with the description of the study; personal questions about age, gender, clinical experience and research experience. One image was enclosed showing a WSL in the distal surface of a posterior tooth neighboring a tooth receiving operative treatment. Patient's characteristics was given in the presentation and illustrated that the patient had high caries risk. The participants were asked two questions: (Q1) if they had seen the situation before, and (Q2) what would be their treatment choice. Six treatment categories were presented varying from no-treatment to non- or operative treatments. Fisher's exact test was performed in the statistical data analysis $(\mathrm{p}<0.05)$. All the 36 dentists had seen the clinical situation before. None of the dentists scored 'no treatment' while $58 \%$ chose fluoride varnish, $25 \%$ chose infiltration, $14 \%$ chose instruction in dental floss/use of tooth picks, and $3 \%$ chose operative treatment. Age, gender, clinical experience, and participation in previous research studies did not influence the results ( $\mathrm{p}$-values $>0.05$ ). All the dentists agreed that the lesion needed treatment and most frequently non-operatively. However, it is necessary to establish a treatment protocol to assess the best treatment for WSL with an open cavity on the neighboring tooth.

\author{
137 \\ Pulse Oximetry of Dental Pulp in Permanent Teeth \\ with Various Stages of Dental Caries \\ M. Groselj*a , K. Cankar b. L. Topolovec ${ }^{c}$, L. Nemeth ${ }^{\mathrm{a}}$ \\ maja.groselj@mf.uni-lj.si \\ aDepartment of Dental Diseases and Normal Dental \\ Morphology, Faculty of Medicine, University of Ljubljana,

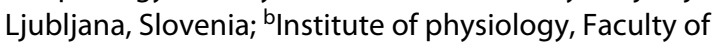 \\ Medicine, University of Ljubljana, Ljubljana, Slovenia; ${ }^{\circ}$ Faculty \\ of Medicine, University of Ljubljana, Ljubljana, Slovenia
}

The assessment of pulp vitality is based mainly on sensitivity testing measuring the sensitivity of pulpal nerves but not pulpal circulation. A more accurate assessment of pulp vitality would be by determining the presence of a functioning blood supply.

The aim of our study was to measure pulp tissue oxygenation in permanent teeth with various stages of dental caries with pulse oximetry, a well-established and noninvasive method for measuring vascular health. Ninety teeth (47 incisors and canines, 27 premolars, 16 molars) were examined by one calibrated examiner. Carious lesions were assessed according to the ICDAS. Pulp vitality was tested with conventional cold test and electric pulp test. Hemoglobin oxygen saturation level of pulp tissue was measured using pulse oximeter (SpectrO2, Smiths Medical). Intact teeth of the same tooth type served as positive controls and endodontically treated teeth as negative controls. Hemoglobin oxygen saturation levels in permanent teeth of different tooth types and with various stages of dental caries were compared using t-test. In endodontically treated teeth no hemoglobin oxygen saturation was detected, other examined teeth had positive clinical sensitivity test recordings. In incisors and premolars lower hemoglobin oxygen saturation levels were found in carious compared to intact teeth $(82.5 \pm 7.6 \%$ vs. $84.1 \pm 8.0 \%, p=0.503)$. Lower hemoglobin oxygen saturation levels were also found in carious premolars compared to intact premolars $(82.4 \pm 7.7 \%$ vs. $86.4 \pm 6.3 \%, \mathrm{p}=0.165)$. In contrast, higher hemoglobin oxygen saturation levels were found in carious molars compared to intact molars $(92.2 \pm 2.8 \%$ vs. $88.4 \pm$ $6.2 \%, p=0.186)$. In conclusion, consistent pulse oximeter readings 
confirm that pulp circulation and pulp tissue oxygenation can be detected by the pulse oximeter. Pulse oximetry is an objective and accurate method of assessing pulp vitality.

Funded by the Slovenian research agency programme P3-0374 Investigation of orofacial health and P3-0019 Applicative and basic physiology and pathophysiology in medicine.

\section{8 \\ What is the Best Method for Detecting Caries Lesions? Systematic Review and Meta-Analysis}

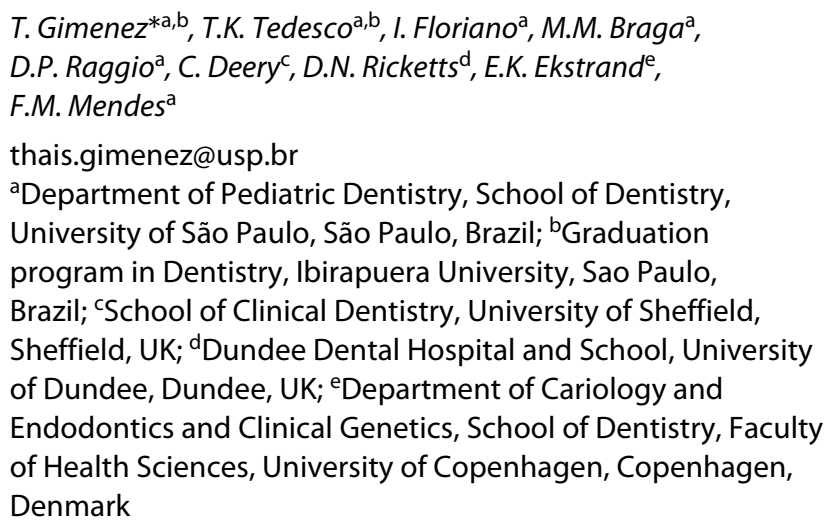

We performed a systematic review and meta-analysis to compare visual, radiographic and fluorescence based methods for detecting carious lesions. Two reviewers searched PubMed, Embase, Scopus and other sources through August 2017 to identify published and non-published studies in English. Studies of visual inspection, radiographic and fluorescence based methods (1) which have assessed accuracy of the method in detecting caries lesions; (2) were performed on occlusal, proximal or free smooth surfaces in primary or permanent teeth; (3) with a reference standard; and (4) which have reported sufficient data about sample size and accuracy of methods were included. The data were used to perform bivariate Summary Receiver-Operating Characteristics curve (SROCs). Meta-regressions and comparisons of SROCs were performed to evaluate differences among caries detection methods. Three hundred and fifty manuscripts from 12,863 articles initially identified and 1 abstract met the inclusion criteria. Analysis demonstrated that the visual inspection showed better results compared to radiographic methods on occlusal surfaces of permanent and primary teeth at initial caries lesions threshold and occlusal surfaces of permanent teeth at advanced caries lesions threshold. In other subgroups and comparisons analyses, there were no statistical significant differences. In conclusion, visual caries detection method is adequate to be used alone in daily clinical practice independently of type of tooth or surface.

Funded by Conselho Nacional de Desenvolvimento Científico e Tecnológico (CNPq), Fundação de Amparo à Pesquisa do Estado de São Paulo (FAPESP - Process 2012/17888-1) and CAPES.

\section{9 \\ Assessment of Caries Status According to CAST Index in Children Living in a High Fluoride Area}

E. Haznedaroğlu*a, Ş. Güner Onur ${ }^{\mathrm{b}}$, B.I. Sezgin ${ }^{\mathrm{a}}$, A.E. Okutan ${ }^{\mathrm{a}}$, G.Ç. Ildeşa ${ }^{\mathrm{a}}$ A. Menteş ${ }^{\mathrm{a}}$

ehaznedaroglu@marmara.edu.tr

aDepartment of Paediatric Dentistry, Marmara University,

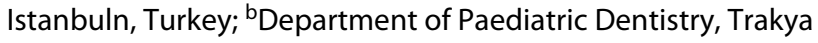

University, Edirne, Turkey

The aim of the study was to evaluate dental fluorosis and caries experience of children living in a village with high fluoride level and compare with a control group living in the same area with low fluoride level. Previously we identified a small village (Hanliyenice, 257 residents) that has a water fluoride level of $2.35 \mathrm{ppm}$, we screened all 30 resident children (17 girls, 13 boys; aged 7 to 12) who attended to the local primary and junior high schools. In the main town (Lalapaşa) and we chose 30 children with age and sex matched as a control group who were exposed to a fluoride level of $<0.3 \mathrm{ppm}$. TF and CAST indices were used to classify dental fluorosis and dental caries status of the children by three calibrated paediatric dentists. The two groups were compared statistically using Mann Whitney U test in SPSS program. The kappa values for the inter-examiners reliability were 0.89 for the TF and 0.92 for the CAST indices. Out of 474 permanent teeth of the children living in Hanliyenice TF0, TF1-2, TF3-4, TF5-8 scores were 155 (32.7\%), $129(27.2 \%), 88(18.5 \%), 102(21.5 \%)$ respectively. The children in the control group had no dental fluorosis (TF0). The CAST results of children from Hanliyenice were statistically higher for CAST 0 (Out of 719 teeth, Fluorosis group 624 teeth (86.8\%); out of 710 teeth, Control group 558 teeth $(78.6 \%) \mathrm{p}=0.034)$ and lower for CAST 4 (Fluorosis group 18 teeth (2.5\%), Control group 43 teeth $(6.1 \%) \mathrm{p}=0.046)$, CAST 6 (Fluorosis group 3 teeth $(0.4 \%)$, Control group 19 teeth $(2.7 \%) \mathrm{p}=0.035)$, CAST $3+4$ (Fluorosis group 59 $(8.2 \%)$, Control group 96 teeth $(13.5 \%) \mathrm{p}=0.025)$ and CAST $5-8$ (Fluorosis group 18 teeth $(2.5 \%)$, Control group 52 teeth $(7.3 \%)$ $\mathrm{p}=0.032$ ). Although there was no statistically difference for total DMFT + dft (Fluorosis group $1.20 \pm 1.82$, Control group $1.83 \pm$ $2.38 ; \mathrm{p}=0.265$ ), when non-cavitated lesions were included (CAST $3+4)$ we found a statistically difference between groups (Fluorosis group $3.17 \pm 2.69$, Control group $5.03 \pm 3.52 ; \mathrm{p}=0.035$ ). In conclusion the results showed that a level higher than optimal fluoride seemed to reduce caries progression in our small sample of children and CAST index exhibited such difference more accurately. 


\section{0 \\ The Utility of Adjunct Methods in Detecting Non-Cavitated Approximal Caries Lesions in Primary Molars}

B.L.P. Moro*a, T.F. Novaes ${ }^{\mathrm{b}}$, R. Matos ${ }^{\mathrm{c}}$, M.M. Braga ${ }^{\mathrm{a}}$, K.R. Ekstrand $^{\text {d }}$,F.M. Mendes ${ }^{\text {a }}$

bruna.moro@usp.br

aDepartment of Pediatric Dentistry, School of Dentistry,

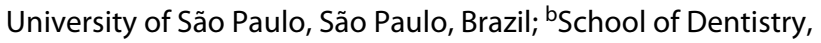
University Cruzeiro do Sul, São Paulo, Brazil; 'School of Dentistry, Uniararas, Araras, Brazil; dSection of Cariology, Endodontics, University of Copenhagen, Copenhagen, Denmark

This study aimed to evaluate the utility of radiographic and pen-type laser fluorescence device (LFpen) as adjunct methods of visual inspection in detecting non-cavitated approximal caries lesions in primary molars. Two calibrated examiners separately performed the evaluations using visual inspection (ICDAS), radiographic and LFpen (DIAGNOdent pen ${ }^{\circledR}$ ) methods. A total of 1,213 approximal surfaces were evaluated in a random sample 126 children (4 to 12 years old) who sought dental treatment. As reference standard, two other examiners checked the surfaces by direct visual inspection after the temporary separation with orthodontic rubbers. Sensitivity, specificity, accuracy and utility 95\% confidence intervals $(95 \% \mathrm{CI})$ of the combination of the methods used for the detection of non-cavitated approximal caries lesions were analysed and compared with visual inspection alone. Sensitivity and specificity values of radiographic and LFpen methods combined with visual inspection simultaneously and sequentially presented similar values than visual inspection alone. The same trend was observed considered accuracy and utility (respectively, 0.656 and 86 for visual inspection, and 0.691 and 87 for the simultaneous combination of visual and radiographic method). The other combinations also presented similar values. The prevalence of noncavitated approximal caries lesions in the sample was $60.5 \%$. When we extrapolated our results for populations with other prevalence values, in any condition the methods combined presented similar accuracy or utility when compared with visual inspection alone. In conclusion, although the adjunct methods have been used to improve the caries diagnostic in the daily clinical practice, its association with the visual inspection did not present advantages in comparison to the clinical examination performed alone for the detection of initial approximal caries lesions in primary molars.

This study was supported by Conselho Nacional de Desenvolvimento Científico e Tecnológico $(\mathrm{CNPq})$ and Fundação de Amparo à Pesquisa do Estado de São Paulo (FAPESP).

\section{1 \\ Pulp Polyp Formation is Linked with Low Carious Lesion Activity}

S. Demant*, L. Bjørndal

sdnp@sund.ku.dk

Department of Cariology and Endodontics, University of

Copenhagen, Copenhagen, Denmark

The aim of this study was to investigate the macroscopic and histological features of pulp polyps related to the progression of deep and extreme deep carious lesions. The material consisted of 68 teeth with progressing stages of deep and extreme deep carious lesions. The teeth were extracted as part of general treatment. The material was scored according to penetration and lesion activity. This was done by evaluating penetration depth radiographically, and activity based on number of surfaces involved in the lesion (macroscopically (1-5) and radiographically (0-3)), as well as discoloration of the demineralized dentine both centrally and in the periphery of the lesions (light/dark). Pulp polyps were scored on sectioned tooth halves of each tooth. In addition, specimens were processed for histology. The association between variables was carried out using Goodman and Kruskal $\gamma$ coefficients. The frequency of pulp polyps was high $(32.4 \%, \mathrm{n}=22)$. When a pulp polyp was present the discoloration of the demineralized dentine in the peripheral lesion area was darker $(\gamma=0.612 ; \mathrm{p}=0.004)$ as well as when the pulp was exposed $(\gamma=0.614, p=0.001)$. Initial stages of polyps could be seen penetrating the roof of the pulp. In later stages the pulp was completely exposed with the lateral borders of surrounding dentine following the dentinal tubules, showing neither signs of bacteria nor yellowish demineralized dentine. In conclusion, our study confirms that penetration to the pulp is a prerequisite for pulp polyp development. Furthermore pulp polyp development seems to be related to more inactive stages of caries lesion activity. It remains unknown why extremely deep caries lesions sometimes lead to pulp polyp formation and in other cases pulpal necrosis.

Funded by the University of Copenhagen Danish Dental Association.

\section{2 \\ Pulpal Laser Doppler Flux in Permanent Teeth with Various Stages of Dental Caries}

L. Nemeth*a, M. Groselja , L. Birk' ${ }^{\mathrm{b}}$, K. Cankar

lidija.nemeth@mf.uni-lj.si

aDepartment of Dental Diseases and Normal Dental

Morphology, Faculty of Medicine, University of Ljubljana,

Ljubljana, Slovenia; ${ }^{b}$ Faculty of Medicine, University of

Ljubljana, Ljubljana, Slovenia; Institute of physiology, Faculty

of Medicine, University of Ljubljana, Ljubljana, Slovenia

Diagnosing dental pulp vitality is based mainly on clinical sensitivity testing. Laser Doppler (LD) fluxmetry represents a method for assessing tissue microvascular function. The method might enable detecting changes in pulpal laser Doppler flux as a part of tis- 
sue response to noxious stimuli, such as dental caries. The aim of this preliminary study was to measure dental pulp tissue LD flux of teeth with various stages of caries progression. Adults with carious lesions and without previous endodontic treatment or prosthetic restoration of permanent teeth were examined. Carious lesions were assessed according to ICDAS criteria by one calibrated examiner. Intact teeth of the same tooth type served as controls. An alginate impression of the tested jaw was made. An individual silicone LD fluxmetry probe holder was designed on the working cast for each patient. Pulpal blood flow of included teeth was was measured using Periflux P4001 Master/4002 Satellite LD monitor (Perimed, Sweden). Laser-Doppler flux values obtained in teeth with different caries stages according to ICDAS system $(\mathrm{N}=12)$ were compared to values of teeth without caries $(\mathrm{N}=11)$ by one way ANOVA (Bonferroni post-hoc test).

There were no statistically significant differences in LD flux values among intact teeth $(\mathrm{N}=16)(8.71 \pm 4.4 .0 \mathrm{PU})$, carious teeth with ICDAS score $2(\mathrm{~N}=3)(7.90 \pm 2.4 \mathrm{PU})$, and teeth with ICDAS score $3(\mathrm{~N}=11)(6.54 \pm 4.5 \mathrm{PU})$. In contrast, there was a significantly increased LD flux in carious teeth with ICDAS score $4(\mathrm{~N}=$ 9) $(12.57 \pm 3.4 \mathrm{PU})$ compared to values obtained in teeth with ICDAS score 3 (Bonferroni test, $\mathrm{p}=0.007$ ) possibly as a consequence of hyperaemia after transitory vasoconstriction.

Funded by the Slovenian research agency programme P3-0374 Investigation of orofacial health and P3-0019 Applicative and basic physiology and pathophysiology in medicine.

\section{3 \\ Molar Incisor Hypomineralization and Dental Caries in Asthmatic Children in Northern Iran}

\author{
K. Salem*, G. Lotfi ${ }^{b}$, T. Cheraqic \\ katayoun.salem@gmail.com \\ aDepartment of Pediatric Dentistry, Azad University of Medical \\ Sciences, Dental School, Tehran, Iran; ${ }^{\text {b}}$ Dentist, Ardabil, \\ Iran; 'Department of Pediatric Asthma and Allergy, Guilan \\ University of Medical Sciences, Rasht, Iran
}

Molar incisor hypomineralization (MIH) is a kind of enamel defect that describes the clinical appearance of enamel hypomineralization of systemic origin. The etiology is unclear. Many risk factors such as respiratory diseases and asthma during the first three years of child's life have been reported. The purpose of this study was to investigate the association between asthma and molar incisor hypomineralization in 6-12 years old children in Rasht city (Guilan province) in north of IRAN. Children (73 asthma patients and 73 controls), 6-12 years of age were enrolled in the study. The diagnosis of asthma was performed by asthma specialist using standard asthma guidelines. The onset of asthma in all asthma patients had been before the age of 3. Medical records was collected using structured questionnaires. Dental examinations were conducted at school and pediatric asthma and allergy clinic on wet teeth using disposable mirror, explorer and head light using DMFS criteria. Statistical analysis was performed using the SPSS21. Chi square test, Fisher's exact test, Independent t-test, Mann-Whitney Test, Multivariate Logistic Regression were used for statistical analysis. The kappa value for inter-examiner reliability and intra- examiner reliability were 0.99 and 0.91 respectively. $29.3 \%$ of asthmatic patients had MIH defects, whereas $17.1 \%$ of control group had this defects. Compared with control group, $\mathrm{MIH}$ was more prevalent in asthmatic children at tooth level [OR $=2.7$ (95\% CI: 1.97-3.68); $\mathrm{P}<0.0001)$ ]. Presence of deciduous molar hypomineralization $(\mathrm{DMH})$ was a risk factor of developing $\mathrm{MIH}(\mathrm{OR}=1.35)$. The Odds Ratio for MIH based on yellow/brown color DMH was 0.42 [(95\% CI: 0.26-0.74); P < 0.01] and on white/creamy color DMH was 0.74 [(95\% CI: 0.52-1.05). $\mathrm{P}=0.05]$. The mean DMFS in asthmatics and controls were $0.34 \pm 0.74$ and $0.45 \pm 1.1$ respectively, $\mathrm{P}=0.9$. In conclusion, pediatric patients with asthma are at increased risk of $\mathrm{MIH}$ and therefore require early dental care. Presence of deciduous molar hypomineralization (DMH) could be an early predictor of MIH.

\section{4 \\ CRA Grid: Preliminary Development and Calibration of a Paper Based Objectivization of Caries Risk Assessment}

U. Sathyanarayanan*, S. Ramarao

candeusha@gmail.com

Department of Conservative Dentistry and Endodontics, Indira Gandhi Institute of Dental Sciences, Sri Balaji Vidyapeeth, Puducherry, India

Caries Risk Assessment (CRA) varies between students and faculty due to subjectivity. The aim was to develop an objectivised paper based, grid system and to assess the rating agreement with the critical thinking process of the teachers. Method: Based on the currently available evidence, specific weightages were assigned to the factors in the existing CRA form. A $10 \times 10$ paper grid was created incorporating these weightages. Shading and summing up the respective squares, provided a numerical score. The range of score indicating the risk status was ascertained by calibrated authors using previous clinical data. After training, the students did CRA for 57 patients by using the Grid. Six faculty were blinded to these grid scores and assessed the risk by routine critical thinking process. Cohen weighted kappa was done using Graph Pad QuickCalcs. Mean percentage of agreement of the six faculty was $79.6 \%$ and with caries grid, was $80.5 \%$. Cohen weighted kappa for agreement at $95 \%$ confidence interval, between the caries grid and faculty were : 0.76 (CI 0.468 to 0.815 ), 0.70 (CI 0.319 to 0.691 ), 0.88 (CI 0.680 to 0.969 ), 0.65 (CI 0.334 to 0.713 ), 0.80 (CI 0.519 to 0.880 ) and 0.63 (CI 0.271 to 0.697 . In this pilot study it can be concluded that there was a good to very good agreement between the risk status assigned by the students using the CRA grid and critical thinking process of the faculty members. 


\section{5}

Caries Risk Assessment: Volatile Organic Compound Analysis

\author{
A. Slimani, E. Terrer, C. Thonat, F. Chaubron, B. Pelissier, \\ I. Panayotov, A. Desoutter, F. Cuisinier, H. Tassery* \\ herve.tassery@univ-amu.fr \\ LBN, University of Montpellier, Montpellier, France
}

The aim of this in vivo study was to analyze the salivary volatile organic compounds (VOC) of two specific populations: one with a low risk of caries (G1) and one with a very high risk of caries (G2) selected using CAMBRA. The positive hypothesis stated that VOC analysis is able to discriminate the caries risk. Stimulated saliva of 30 subjects for each group was collected using $4 \mathrm{ml}$ of tartrazin solution before stabilization with azide sodium and ammonium sulfate. $3 \mathrm{ml}$ of pooled saliva from each subject were analyzed by solid phase microextraction linked to gas chromatography-mass spectrometry (SPME-GC-MS). 109 individual volatile compounds were identified in total in the saliva samples and the concentrations of these in the two groups were compared using ANOVA according to the factor "caries risk" with Statistica 13 software. Eleven VOCs (1,2,4-trimethyl cyclopentane; ethanol; 2-ethyl furane; hexane; 2-methyl pentane; toluene; 3-methyl pentane; 4,4-dimethyl2-pentene; methylcyclopentane; 2,3-butanedione; cyclohexane) were significantly different between the groups and these were used in Principal Component Analysis (PCA) in order to was made on these 11 VOCs, the two first axes accounted $86 \%$ of the information and PCA profiles generated two visually distinct clusters. A subset discriminant analysis was performed to select the best combination of three VOCs leading to the greatest discrimination of the two studied groups. The most discriminating VOCs were cyclohexane, ethanol and methylcyclopentane.

\section{6 \\ Clinical Guideline for Tooth Separation to Improve Assessment of Approximal Caries Lesions: An Educational Report}

\author{
M.M. Nascimento*a, M.E. Ottenga ${ }^{\mathrm{b}}$, A.J. Delgado ${ }^{\mathrm{a}}$, \\ M.G.D. Caraballo , A.P. Ribeiro ${ }^{\text {a }}$, L. Cassiano a, J.F. Roulet ${ }^{\text {, }}$ \\ S. Geraldelia, P.N.R. Pereira ${ }^{\mathrm{a}}$, D.A. Dilbone \\ mnascimento@dental.ufl.edu \\ aDepartment of Restorative Dental Sciences, Division of \\ Operative Dentistry; College of Dentistry, University of Florida, \\ Gainesville, FL, USA; bepartment of Restorative Dentistry, \\ LECOM School of Dental Medicine, Bradenton, FI, USA
}

In the era of tooth-preserving dentistry, restorative intervention of caries lesions should only be considered after non-invasive efforts are proven ineffective at controlling caries activity. In particular, placement of restorations on cavitated approximal lesions is deemed appropriate as cavitation on approximal tooth surfaces allows accumulation and metabolic activity of oral biofilms, thus allowing lesion progression. However, visual-tactile assessment of cavitation on approximal surfaces can be challenging due to the restricted access to contacting surfaces, and bitewing radiographs may not reveal the surfaces' integrity with accuracy. Consequently, radiographic lesion depth or progression into dentin has become the main threshold criterion for restorative intervention of approximal lesions. Yet, earlier studies using tooth separation showed that nearly two-thirds of lesions detected radiographically at the outer half of dentin are not cavitated. Inaccurate assessment of cavitation status resulting in unnecessary restorations is of great concern. This report describes the implementation of a clinical guideline for tooth separation to improve visual-tactile assessment of approximal lesions at the UF College of Dentistry. Implementation stages included faculty engagement, calibration, and testing of technique feasibility, potential diagnostic value, and patient acceptance. Importantly, a rigorous protocol was developed for careful documentation and monitoring of non-cavitated lesions using electronic dental records. The guideline mandates the use of orthodontic bands for temporary tooth separation of approximal lesions presenting no evident cavitation and extending radiographically to the outer third of dentin (D1). Preliminary data revealed that $100 \%$ of E1 $(n=25), 89.7 \%$ of E2 $(n=39), 57.1 \%$ of D1 $(n=$ 28 ), and $36.4 \%$ of D2 lesions were non-cavitated after separation, and these lesions are being managed by non-invasive measures. This clinical and electronic-based guideline supports the use of tooth separation as an effective diagnostic aid for assessment and appropriate management of approximal caries lesions.
147
The Development of a Theory-Based Mobile-Health Intervention to Improve Oral Hygiene During Orthodontic Treatment
J.F.M. Scheerman ${ }^{\mathrm{a}-\mathrm{c}}$, P. Van Empelen ${ }^{\mathrm{c}}$, B. Van Meijela , J. Lamme ${ }^{\mathrm{a}}$, C. Van Loveren*b
c.van.loveren@acta.nl
anholland University of Applied Sciences, Amsterdam, The
Netherlands; ${ }^{b}$ Academic Centre for Dentistry Amsterdam
(ACTA), Amsterdam, The Netherlands; ' ${ }^{C}$ etherlands
Organization for Applied Scientific Research (TNO), Leiden,
The Netherlands

The aim of this study was to describe the systematic development of a theory-based mobile-health (mHealth) intervention aimed to prevent dental caries among adolescents with fixed orthodontic appliances. The intervention mapping protocol was used to develop the mHealth intervention. In this study, five steps of intervention mapping (1) problem analysis, (2) program objectives and outcomes, (3) program design (4) program production and (5) program implementation. On the basis of our problem analysis (step 1), we identified fluoride use and the control of dental plaque were identified as target behaviours. The Health Action Process Approach (HAPA) theory showed to be applicable in explaining differences in oral-health behaviours in our target group. After defining program objectives and outcomes (step 2), we selected behaviour change methods targeted at psychosocial factors of HAPA including: self-monitoring of dental plaque and behaviour (by using disclosing tablets and taking selfies), goal-setting and implementation intentions (if-then planning), sending re- 
minders (step 3). This resulted in the WhiteTeeth app (in the appstore 'Witgebit') (step 4). The app was piloted for acceptability and usability in a group of 28 peers. The mean system usability scale (SUS) was 77, which means an acceptable score. Meetings with the target group and dental professionals were organized to prepare for the implementation (step 5). The most important finding was that they liked and appreciated the app. This comprehensive description of the content and systematic development of the app responds to recent calls for improved reporting on the process of intervention development.

\section{8 \\ The Effectiveness of the WhiteTeeth App on Oral Hygiene - A Randomized Controlled Trial \\ J.F.M. Scheerman*a-c, M.C.T. Van den Braak ${ }^{\text {b }}$, G.H.W. Verrips', P. Van Empelen ${ }^{c}$, B. Van Meijel', L. Somi ${ }^{\mathrm{a}}$, M. Boezewinkela, C. Van Loveren ${ }^{\text {b }}$, G. Kramer \\ Janneke.scheerman@inholland.nl \\ anholland University of Applied Sciences, Amsterdam, The Netherlands; ${ }^{b}$ Academic Centre for Dentistry Amsterdam (ACTA), Amsterdam, The Netherlands; ' Netherlands Organization for Applied Scientific Research (TNO), Leiden, The Netherlands}

The objective was to evaluate the effect of the WhiteTeeth app, an evidence- and theory-based mobile health (mHealth) intervention, on oral hygiene. The app provided personal oral-health advice and an automatic-coaching program. In this parallel randomized controlled trial, 132 eligible Dutch adolescents with fixed orthodontic appliances were recruited from two orthodontic clinics. Data were collected during three orthodontic check-ups: baseline, 6-weeks follow-up, and 12-weeks follow-up. Adolescents were randomly allocated to two groups: (1) the intervention group was given access to the WhiteTeeth app in addition to usual care and (2) the control group only received usual care. The degree of oral hygiene was determined by the amount of dental plaque (measured with a modified Silness and Loë Plaque Index) and the presence of gingival bleeding (measured with the Bleeding on Marginal Probing Index). Linear mixed models were used to measure the effectiveness of the app. After 12-weeks follow-up, the participants who used the WhiteTeeth app additionally to usual care showed a significant improvement in the amount of dental plaque (Crude $\beta=-10.73, p=0.004$; Adjusted $\beta=-16.68, p=0.019$ ) and in the presence of gingival bleeding (Crude $\beta=-2.39, p=0.057$; Adjusted $\beta=-5.42, p=0.031$ ) compared to those who received usual care only. In conclusion the results support that offering personal oral-health advice and automatic coaching through an mHealth intervention is an effective means to support adolescents with fixed orthodontic appliances improving their oral hygiene.

The study was financed by ACTA and Inholland University.

\section{9}

Are Standardised Caries Risk Assessment Models Effective in Assessing Caries Increment? A Systematic Review

\author{
M.G. Cagetti ${ }^{\mathrm{a}, \mathrm{b}}$, G. Bontà ${ }^{\mathrm{a}}$, F. Cocco ${ }^{\mathrm{b}, \mathrm{c}}$, C. Ricciardi*a, \\ L. Strohmenger ${ }^{\mathrm{a}, \mathrm{b}}$, P. Lingström ${ }^{\mathrm{b}, \mathrm{d}}, \mathrm{G} \mathrm{Campus}^{\mathrm{b}, \mathrm{c}}$ \\ camilla@camillaricciardi.it \\ aDepartment of Biomedical, Surgical and Dental Sciences,

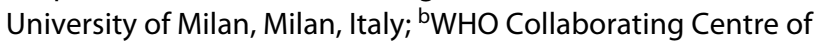 \\ Milan for Epidemiology and Community Dentistry, University \\ of Milan, Milan, Italy; ' Department of Surgery, Microsurgery \\ and Medical Sciences, School of Dentistry, University of Sassari, \\ Viale San Pietro 43/C, I-07100 Sassari, Italy; ${ }^{\text {d Department of }}$ \\ Cariology, Institute of Odontology, Sahlgrenska Academy, \\ University of Gothenburg, Gothenburg, Sweden
}

A systematic review was performed to evaluate whether standardised caries risk assessment (CRA) models are able to judge the risk according to the actual caries status and/or the future caries increment. PubMed, Scopus and Embase were searched from 2000 to 2016. A search filter was developed. All the papers meeting the inclusion criteria were subjected to a quality assessment, based on customised quality assessment tools developed by National Heart, Lung, and Blood Institute and Research Triangle Institute International. Results. 1,900 papers were identified and, after revision, 32 were included. In all but one, the Cariogram was used as the only model or in comparison with other models. All the papers on children $(\mathrm{N}=16)$ found a statistically significant association between the risk levels and the actual caries status and/or the future caries increment. Eleven papers, all of them including the Cariogram, were classified as being of good quality. The same positive association was reported in papers on adults $(\mathrm{N}=12)$. Eight papers were classified as being of good quality and all except one used the Cariogram. Three of four papers comprising children and adults found a positive association. Six of the included papers, five on children and one on adults, report data on sensibility and specificity of the CRA models; data show that Cariogram reliability is fair and differs in the different caries risk levels. The scientific evidence relating to standardised CRA models is still limited. Only the Cariogram has been tested to conclude that the method might be effective in caries assessment and prediction. 


\section{0 \\ Quality of Saliva in Diabetes Patients Either with or without Xerostomia}

\author{
A. Saeed, A.L. Kahtani ${ }^{\text {, }}$ A. Sleibi ${ }^{\mathrm{a}}$, G. Carpenter ${ }^{\mathrm{b}}$, P. Anderson ${ }^{\mathrm{a}}$, \\ A. Baysan*a \\ a.baysan@qmul.ac.uk \\ 'Barts and the London, School of Dentistry, QMUL, London,

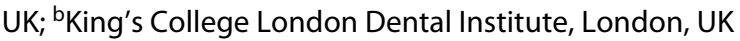

Diabetes mellitus is known to affect the salivary glands and causes various changes in salivary chemical and physical properties. Xerostomia is frequently associated amongst diabetes and cause several side effects to oral and general health. The aim of this study was to assess the physical and chemical properties of saliva collected from diabetic patients. There were three groups involved in this study; patients with diabetes and xerostomia (DX, $\mathrm{n}=5$ ); patients with diabetes without xerostomia $(\mathrm{DN}, \mathrm{n}=5)$; and healthy participants $(\mathrm{H}, \mathrm{n}=5)$ without diabetes and xerostomia. Participants were initially screened according to their unstimulated whole saliva (USWS) which was $<0.2 \mathrm{ml} / \mathrm{min}$ for DX, whilst USWS $>0.2 \mathrm{ml} / \mathrm{min}$ for both $\mathrm{DN}$ and $\mathrm{H}$. A total of 15 unstimulated and stimulated whole mouth saliva samples was collected. The matched-age ranged from 35 to 58 years. For each sample, $\mathrm{pH}$ and buffer capacity were measured using saliva kit (GC, Japan), and saliva Spinnbarkeit using NEVA METER (IMI-021, Ishikawa Ironworks Co., Ltd., Japan). The results showed that USWS pH was: DX, $6.52 \pm 0.65 ; \mathrm{DN}, 7.00 \pm 0.9 ; \mathrm{H}: 7.24 \pm 0.35$, whilst stimulated whole saliva $\mathrm{pH}$ was: $\mathrm{DX}, 7.80 \pm 0.26 ; \mathrm{DN}, 7.7 \pm 0.08$; and $\mathrm{H}$, $7.7 \pm 0.8$. (ANOVA, $p=0.036$ ). The USWS buffer capacity was for DX: $5.20 \pm 1.48$, DN: $7.00 \pm 2.78$ and $\mathrm{H}: 7.60 \pm 1.50$. In addition, SWS buffer capacity was DX: $10.00 \pm 1.80, \mathrm{DN}: 10.40 \pm 1.30, \mathrm{H}$ : $11.20 \pm 1.78)$. Saliva Spinnbarkeit results were from DX: $2.99 \pm$ $0.17, \mathrm{DN}: 2.79 \pm 0.02$ and for $\mathrm{H}: 4.3 \pm 3.450 .035$ ). This study showed that participants with xerostomia tended to have lower USWS $\mathrm{pH}$, since the $\mathrm{pH}$ level is associated with salivary flow rate. The saliva Spinnbarkeit had also lower values in diabetes patients when compared to the healthy participants.

Part of the research project for MSc in Oral Biology.

\section{1 \\ Immunohistochemical Study on T and B Limphocytes in Human Dental Pulp}

\author{
A. Sotirovska Ivkovska*a, E. Zabokova-Blbilova a , Z. Georgieva, \\ V. Ambarkova ${ }^{\text {, }}$ N. Stavreva ${ }^{\text {b }}$, A. Mijoska ${ }^{\text {b }}$ \\ anasotirovska@yahoo.com \\ aDepartment of Pediatric and Preventive Dentistry, Faculty \\ of Dentistry, Skopje, Republic of Macedonia; ${ }^{b}$ Department \\ of Prosthodontics, Faculty of Dentistry, Skopje, Republic of \\ Macedonia
}

This study was undertaken to show the presence of immune components in the dental pulp and to determine how and which immunocompetent cells infiltrate the pulp in association with the development of carious lesions. In this study we have analysed 150 teeth with different stages of progression of the carious lesion. The condition of the pulp was classified into five groups according to the progression of the carious lesions from stages S0 (non-carious teeth) to S4 (exposed pulp). Teeth were extracted and immediately cut longitudinally; pulp tissue was extirpated and fixed in formalin for 24 hours at $4{ }^{\circ} \mathrm{C}$. The specimens were embedded in paraffin, according to standardized laboratory procedure. Sections were cut at $5 \mu \mathrm{m}$ thicknesses and stained by the streptavidin - biotin complex immunoperoxidase method. Cells were identified with indirect immunoperoxidase technique using monoclonal antibodies reactive to pan-T lymphocytes, helper (CD4), suppressor (CD8) lymphocytes and B-lymphocytes. T lymphocytes were observed in unaffected pulp with suppressor (CD8) lymphocytes being predominant. In S1 and S2 a focal accumulation of mononuclear inflammatory infiltrate was detected, with the ratio of CD4/ CD8 0.22 and 0.23 , respectively. The ratio of $\mathrm{B} / \mathrm{T}$ lymphocytes was 0.21 and 0.24 . Higher numbers of T, CD4, CD8 and B-lymphocytes were observed in S3 with a CD4/CD8 ratio of 0.84 and $\mathrm{B} / \mathrm{T}$ lymphocytes ratio was 0.57 . In the group with exposed pulp a high ratio of $\mathrm{CD} 4 / \mathrm{CD} 8$ and $\mathrm{B} / \mathrm{T}$ lymphocytes was observed (1.22 and 1.81 ), showing signs of irreversible pulp pathosis. This study suggest that helper (CD4) and suppressor (CD8) T lymphocytes have regulatory functions and the interaction of $\mathrm{T}$ and $\mathrm{B}$ lymphocytes and their products have crucial role in the pathogenesis of pulpal disease.

The study was financial supported by Macedonian Ministry of Education. 


\section{2}

\section{Pulp Chamber Temperature Development in a Natural Tooth When Using Different LED Light Curing Units}

M. Mouhat ${ }^{\mathrm{a}}$, L. Stangvaltaite ${ }^{\mathrm{a}}$, J.B. Mercer ${ }^{\mathrm{b}}$, U. Örtengren*a,c

mathieu.mouhat@uit.no

${ }^{a}$ Department of Clinical Dentistry, Faculty of Health

Sciences, UiT The Arctic University of Norway, Troms $\varnothing$, Norway; ${ }^{b}$ Department of Medical Biology, Faculty of Health

Sciences, UiT The Arctic University of Norway, Troms $\varnothing$, Norway; 'Department of Cariology, Institute of Odontology/ Sahlgrenska Academy, Göteborg University, Göteborg, Sweden

Light-cured resin-based composites are widely used in management of carious lesions. Increased pulp chamber temperature (Tpc) by LED light curing units (LCU) may cause pulp damage. We investigated Tpc increment using LED-LCU with different irradiance, irradiance emission mode (IEM) and spectral emission profile (mono/polywave). Eight different LED-LCU were tested (Elipar $^{\mathrm{TM}}$ DeepCure and Elipar ${ }^{\mathrm{TM}}$ S10 (3 m ESPE, Seefeld, Germany), SmartLite ${ }^{\circledR}$ Focus (Dentsply Caulk, Milford, USA), DemitmUltra (Kerr Dental, Orange, USA), TransLux ${ }^{\circledR}$ Wave and Trans-
$\operatorname{lux}^{\circledR} 2$ Wave (Kulzer, Hanau, Germany), Bluephase Style ${ }^{\circledR}$ and Bluephase $\mathrm{G} 2^{\circledR}$ (IvoClar/Vivadent, Schaan, Lichtenstein). LEDLCU irradiance was controlled before each test (MARC Resin Calibrator, BlueLight Analytics ${ }^{\mathrm{TM}}$, Halifax, Canada). An extracted human molar with removed cusps was mounted in a holder suspended in a thermostatically controlled water bath $\left(36.8 \pm 0.2^{\circ} \mathrm{C}\right)$ with the root of the tooth submerged. Using a thermocouple, Tpc measurements were performed for 10 and 20 seconds at $0 \mathrm{~mm}, 2 \mathrm{~mm}$ and $4 \mathrm{~mm}$ distance. Multivariable linear regression analyses were used. Ethical permission was sought but not required. The highest Tpc increment $\left(6.1^{\circ} \mathrm{C}\right)$ was produced by Bluephase $\mathrm{G} 2^{\circledR}$ high mode at $2 \mathrm{~mm} 20 \mathrm{~s}$, while the lowest $\left(0.8^{\circ} \mathrm{C}\right)$ by Bluephase Style ${ }^{\circledR}$ at $4 \mathrm{~mm} 10 \mathrm{~s}$. Time ( $10 \mathrm{~s}$ vs $20 \mathrm{~s})$ was the strongest predictor and increased Tpc by $1.5^{\circ} \mathrm{C}(\mathrm{p}<0.0001)$, followed by irradiance $\left(0.3^{\circ} \mathrm{C}\right.$ Tpc increment every $\left.100 \mathrm{~mW} / \mathrm{cm}^{2}, \mathrm{p}<0.0001\right)$ using polywave LED-LCU. Monowave LED-LCU IEM was the second strongest predictor; pulsatile IEM decreased Tpc by $1^{\circ} \mathrm{C}$ compared to continuous IEM $(\mathrm{p}<0.0001)$. For polywave LED-LCU increased distance resulted in lower Tpc $(\mathrm{p}<0.0001)$, while monowave LEDLCU did not have a significant influence. In conclusion, when choosing LED-LCU clinicians should be aware that there might be other factors than irradiance, which increase Tpc, such as time, IEM and spectral emission profile of LED-LCU.

The study was financial supported by Norwegian Directorate of Health. 


\section{Session 12 Diagnostics: Lab-Based Studies Hard Tissues}

153

\section{Multiphoton Microscopy for Caries Detection}

A. Slimani*a, D. Tardivo b, I. Panayotova , B. Levallois , , C. Gergelyc, F. Cuisinier ${ }^{\mathrm{a}}$, H. Tassery ${ }^{\mathrm{a}, \mathrm{b}}$, T. Cloitre $^{\mathrm{c}}$, E. Terrer $^{\mathrm{a}, \mathrm{b}}$

amel.slimani@umontpellier.fr

aLBN, University of Montpellier, Montpellier, France;

bUniversity of Aix-Marseille, Marseille, France ${ }^{c}$ L2C UMR 5221

CNRS, University of Montpellier, Montpellier, France

Multiphoton microscopy (MPM) is a nonlinear high-resolution technique that can be applied to collagen studies such as collagen type I denaturation during caries process. The MPM lies in its simultaneous image creation based on the fluorescence signals produced by a multiphoton excitation (2-photon excited fluorescence $[2 \mathrm{PEF}]$ ) and a coherent nonlinear phenomenon, second harmonic generation (SHG). This study aims to investigate the structural modifications during the dentin caries process by monitoring the variations of the SHG and the SHG/2PEF ratio recorded by nonlinear optical spectroscopy (NLOS). The International Caries Detection and Assessment System (ICDAS) was used as a clinical reference in vitro. To this end, teeth $(n=35)$ for each ICDAS score were selected and longitudinal sections through the caries lesion were performed. SHG and $2 \mathrm{PEF}$ images and spectra were recorded for each ICDAS score (0 to 6). $2 \mathrm{PEF}$ and SHG images highlighted the microarchitecture and optical properties of the collagen denaturation in dentin during the caries process. The SHG images of carious dentin demonstrated a destruction of the collagen matrix. The variation of intensity of the $2 \mathrm{PEF}, \mathrm{SHG}$ and the ratio SHG/2PEF allowed the merge of ICDAS scores: ICDAS 0/1; ICDAS 2/3; ICDAS $4 / 5 / 6$. The new groups tend to be similar to the International Caries Classification and Management System (ICCMS). The $2 \mathrm{PEF}$ and SHG intensities and the ratio SHG/2PEF are highly altered during the caries process. The ANOVA-parametric test was used to compare the average ratios of these three new groups $(\mathrm{p}=$ $\left.8.88 \times 10^{-9}\right)$ and a significant difference was found $(\mathrm{p}<0.05)$. In conclusion, the ratio $\mathrm{SHG} / 2 \mathrm{PEF}$ is a relevant parameter to monitor the caries evolution and it defined three stages: initial lesion (ICDAS 0/1), moderate lesion (ICDAS 2/3) and extensive lesion (IC-
DAS 4/5/6). The development of a clinical device based on the nonlinear optics can bring a valuable diagnostic tool for caries detection in an everyday practice.

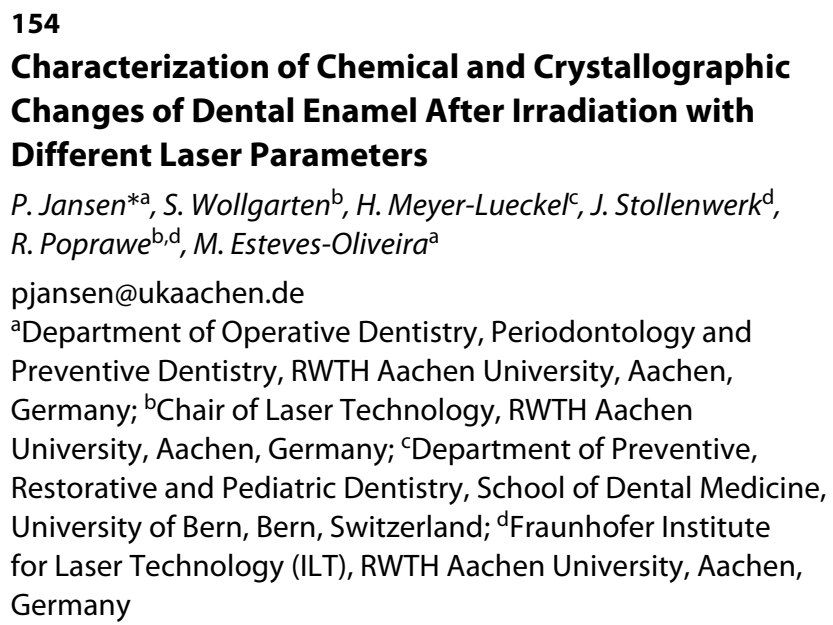

To protect dental enamel from erosion a new laser scanning strategy has been recently established. Although, a significant increase of enamel acid resistance was shown, it is currently yet unknown, which chemical and crystallographic changes of enamel are related to this effect. Therefore, the aim of this study was to identify the chemical and structural changes occuring in enamel after laser irradiation.

Ground polished bovine enamel blocks were randomly divided in six groups: one negative control $(\mathrm{C})$, receiving no treatment, and five laser groups irradiated with parameters causing surface temperatures from ca. 500 to $900{ }^{\circ} \mathrm{C}: 500^{\circ} \mathrm{C}$ [(L500), $33 \mu \mathrm{s} / 2 \mathrm{KHz}$, $600^{\circ} \mathrm{C}$ [(L600), $\left.20 \mu \mathrm{s} / 2 \mathrm{KHz}\right], 700^{\circ} \mathrm{C}$ [(L700), $\left.\left.20 \mu \mathrm{s} / 20 \mathrm{KHz}\right)\right]$, $800^{\circ} \mathrm{C}$ [(L800), $\left.490 \mu \mathrm{s} / 2 \mathrm{KHz}\right], 900^{\circ} \mathrm{C}$ [(L900), $\left.55 \mu \mathrm{s} / 2 \mathrm{KHz}\right](\mathrm{n}=$ 20). $\mathrm{CO}_{2}$ laser irradiation was perfomed at $10.6 \mu \mathrm{m}$ using a meander scanning pattern $(3-126 \mathrm{~mm} / \mathrm{s})$. After the treatments enamel was scraped off the blocks and pulverized until a grain size of $\leq 45 \mu \mathrm{m}$. Mineral phase changes were analysed by XRD ( $2 \theta$-range: 
5-70 $, \mathrm{CuKa}, 40 \mathrm{kV}, 40 \mathrm{~mA}$, step size: $0.01^{\circ}$, counting time: $96 \mathrm{~s}$, Bregg-Brentano geometry) and carbonate content and crystallinity by FTIR (max. resolution: $4 \mathrm{~cm}^{-1}$; range: $400-4000 \mathrm{~cm}^{-1}$; $\mathrm{KBr}-$ method; scan number: ca.500). The Infrared splitting factor (IRSF) was used as surrogate for crystallinity. FTIR data were statistically analysed using one-way ANOVA and post-hoc tests $(\alpha=0.05)$. XRD analysis showed no mineral phase change for L500 and L600, while for L800 and L900 $\alpha$-Tricalciumphosphate and Tetracalciumphosphate were detected. The FTIR analysis showed a significant reduction of carbonate content for all groups ( $p<0.05$ ). L500 and L600 additionally significantly increased enamel crystallinity (IRSF) means compared to non-treated enamel ( $\mathrm{p}<0.05) . \mathrm{CO}_{2}$ laser parameters L500 $\left(33 \mu \mathrm{s} / 2 \mathrm{KHz} / 1.6 \mathrm{~J} / \mathrm{mm}^{2}\right)$ and $\mathrm{L} 600$ $\left(20 \mu \mathrm{s} / 2 \mathrm{KHz} / 1.3 \mathrm{~J} / \mathrm{mm}^{2}\right)$ reduced carbonate content, improved crystallinity and caused no deleterious mineral phase changes, characteristics known to increase enamel acid resistance.

This study was funded by the DFG (German Research Foundation, Grants Nr.: ES 426/2-1, PO 591/40-1).

\section{5 \\ Determining the Efficacy of Potassium Oxalate Containing Strips to Reduce Dentinal Fluid Flow In Situ}

N. Chapman*a, S. Jones ${ }^{\mathrm{a}}$, P. Bahal ${ }^{\mathrm{a}}$, T. He ${ }^{\mathrm{b}}$, P. Drake ${ }^{\mathrm{b}}$, N. West $^{\mathrm{a}}$

nick.chapman@bristol.ac.uk

aClinical Trials Unit, Bristol Dental School, University of Bristol, Bristol, UK; ${ }^{b}$ Proctor and Gamble Company, Mason, OH, USA

It is believed that dentine hypersensitivity is caused by a stimulus being applied to exposed dentinal tubules resulting in a transient pressure change, inducing movement of fluid in the tubules and subsequent activation of mechanoreceptors with a correlated pain sensation. This study aimed to use a flow cell model to measure the efficacy of a potassium oxalate containing gel strip in occluding dentinal tubules before and after housing within the oral environment. Dentine discs $1 \mathrm{~mm}$ thick $(\mathrm{n}=40)$ were sectioned from whole human molars, and the hydrodynamic flow of fluid through the dentinal tubules in a $0.32 \mathrm{~cm}^{2}$ sealed window was recorded at $206 \mathrm{kPa}$. Flow rates were measured at baseline and post 10 minute treatment with a gel strip containing $3.14 \%$ potassium oxalate or no treatment. One treated and one un-treated dentine disc were separately housed in palatal appliances and worn by 20 healthy participants for 14 days, after which the flow rate of the samples was re-measured. Treatment with the potassium oxalate containing strip reduced flow rate from $68.0 \mu \mathrm{l} / \mathrm{min}$ at baseline to $29.3 \mu \mathrm{l} / \mathrm{min}$ which was highly significant (Wilcoxon Signed Rank Test, $\mathrm{p}<0.0001$ ), following housing in the oral environment the flow rate was further reduced to $3.7 \mu \mathrm{l} / \mathrm{min}$. The flow rate of the non-treated discs reduced from $103.2 \mu \mathrm{l} / \mathrm{min}$ at baseline to $16.0 \mu \mathrm{l} /$ min after oral housing which was also highly significant (Wilcoxon Signed Rank Test, $\mathrm{p}<0.0001)$. Following exposure to the oral environment, flow rates were compared using analysis of covariance for split mouth, placebo-controlled design. The discs pre-treated with the potassium oxalate containing strip provided significant reduction in flow rate when compared to the non-treated discs with estimated median of 3.6 and 10.5, respectively. This model was effective in showing the occluding potential of potassium oxalate containing strips in reducing fluid flow through dentinal tubules following treatment, as well as following 14 days of exposure to the oral environment.

The study was funded by Proctor and Gamble Company, USA.

\section{6 \\ Analyzing Direction for Non-Cavitated Approximal-Caries Detection by Optical Coherence Tomography (OCT)}

H. Xing ${ }^{\mathrm{a}, \mathrm{b}}$, G.J. Eckert ${ }^{\mathrm{c}}$, M. Ando*b

mando@iu.edu

aDepartment of General Dentistry, Peking University School and Hospital of Stomatology, Beijing, China; ${ }^{b}$ Department of Cariology, Operative Dentistry and Dental Public Health, Indiana University School of Dentistry, Indianapolis, IN, USA; 'Department of Biostatistics, Indiana University School of Medicine, Indianapolis, IN, USA

The objective was to assess the effect of analyzing the direction of OCT for detection of non-cavitated approximal caries. Thirty human extracted-premolars were selected based on micro-computed tomography [ $\mu-\mathrm{CT}$ : $\mu \mu-\mathrm{CT}=0$ : sound $(\mathrm{n}=12), \mu-\mathrm{CT}=1$ : lesions into outer-half of enamel $(n=6), \mu \mu$-CT $=2$ : lesions into inner-half of enamel $(n=6)$, and $\mu-C T=3$ : lesions into outer onethird of dentine $(n=6)]$. Teeth were mounted in a custom-made device to simulate approximal contact. Each tooth was scanned by OCT from occlusal (marginal-ridge above contact). OCT images were observed and analyzed deepest caries extension (sound, lesions into outer-half of enamel, lesions into inner-half of enamel, and lesions into outer one-third of dentine) using three third of dentine) using three-ways: Cross-section; Coronal-section; and Deepest (deepest of Cross-section and Coronal-section). All measurements were repeated 48-hrs later. Weighted-Kappa, sensitivity, specificity, area under ROC curve (Az) were analyzed. Correlation with Kappa, sensitivity, specificity, area under ROC curve (Az) were analyzed. Correlation with $\mu$-CT was also determined. Sensitivity was further evaluated based on lesion extension. Weighted-Kappas were: Cross-section (0.90), Coronal-section (0.95), and Deepest (0.95). Sensitivity/specificity/Az for Cross-section, Coronal section, Coronal-section, and Deepest were $94 \% / 58 \% / 0.76,81 \% / 100 \% / 0.90$, and $94 \% / 58 \% / 0.82$, respectively. Sensitivities by lesion extension $(\mu \mathrm{CT}=1 / \mu \mathrm{CT}=2 / \mu \mathrm{CT}=3)$ for Cross-section, Coronal section, Coronal-section, and Deepest were $100 \% / 83 \% / 100 \%, 58 \% / 83 \% / 100 \%$, and $100 \% / 83 \% / 100 \%$, respectively. For sensitivity, there were no significant differences in overall $/ \mu \mathrm{CT}=1 / \mu \mathrm{CT}=2 / \mu \mathrm{CT}=3(\mathrm{p} \geq 0.09 / \mathrm{p} \geq 0.06 / \mathrm{p}=1.00 / \mathrm{p}=$ $1.00)$, respectively. For specificity, Coronal-section was significantly higher than Cross section was significantly higher than Cross-section and Deepest $(\mathrm{p}=0.004)$; but Cross-section and Deepest were not significantly different from each other $(p=1.00)$. Cross-section had significantly lower Az than Deepest ( $\mathrm{p}=0.048$ ), but Coronal-section was not significantly different than Cross-section $(p=0.07)$ or Deepest $(p=0.20)$. Correlation coefficients with section $(p=0.07)$ or Deepest $(p=0.20)$. Correlation coefficients with $\mu$-CT were Cross-section $(0.53, \mathrm{p}<0.001)$, Coronal-section 
(0.84, $\mathrm{p}<0.001)$, and Deepest $(0.66, \mathrm{p}<0.001)$. Within the limitations of this study, sensitivity and $\mathrm{Az}$ indicated high values for both analyzing directions, except Coronal section $(0.84, \mathrm{p}<0.001)$, and Deepest $(0.66, \mathrm{p}<0.001)$. Sensitivity and $\mathrm{Az}$ indicated high values for both analyzing directions, except Coronal-section at section at $\mu \mathrm{CT}=1$.

\section{7}

\section{Antibacterial Efficacy of Erythrosine-Containing Caries Detector Dye}

Juhea Chang*a, Shin Hye Chung ${ }^{\text {b }}$

Juhchang@snu.ac.kr

aspecial Care Clinic, Seoul National University Dental Hospital,

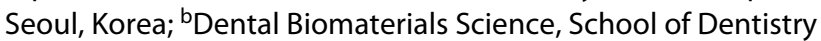
and Dental Research Institute, Seoul National University,

Seoul, Korea

This study aimed to evaluate the antibacterial efficacy of an experimental caries detector dye. The dye containing erythrosine (Merck KGaA, Darmstadt, Germany) [Group 1: erythrosine; Group 2: erythrosine with LED light curing (Elipar DeepCure-S, $1000 \mathrm{~mW}, 20 \mathrm{sec})]$ was compared with two conventional caries detectors (Group 3: Seek Sable, Ultradent, South Jordan, USA; Group 4: Seek, Ultradent). The antibacterial effect on Streptococcus mutans UA159 was evaluated by a bacterial diffusion test. Propylene glycol and $0.1 \%$ chlorhexidine solution were used as negative and positive controls, respectively. The contact angle of the dyes on the enamel surface was measured for the wetting capability. Bacterial growth inhibition was evaluated by CLSM (LSM700, Carl Zeiss Meditec, Jena, Germany) with LIVE/DEAD ${ }^{\circledR}$ BacLightTM Bacterial Viability Kit. Bacterial morphology was evaluated under SEM (S-4700, Hitachi, Tokyo, Japan). Groups 1 and 2 exhibited significantly larger bacterial inhibition zones compared to Groups 3 and $4(\mathrm{p}<0.05)$. The antibacterial activity was enhanced with increased concentrations of erythrosine $(0.001 \mathrm{M}, 0.01 \mathrm{M}$, $0.1 \mathrm{M}$, and $1 \mathrm{M}$ ) and with light curing. CLSM demonstrated noticeably denser fluorescence, indicating less viable cells in Groups 1 and 2 compared to others. SEM showed less bacterial adhesion on the dentinal surface in Group 2. The experimental dye had similar wetting behavior compared to the conventional dyes. Erythrosinecontaining caries detector dye had an antibacterial effect and this effect was increased with light activation.

This work was supported by Creative-Pioneering Researchers Program through Seoul National University.

\section{8 Validation of QLF Scoring System for Assessing the Non-Cavitated Occlusal Caries Lesion}

E.H. Jung*a, S.W. Park' ${ }^{\text {a }}$ H.S. Lee ${ }^{\text {a }}$, M.J. Chung ${ }^{\text {b }}$, J.Y. Na', H.K.KWon ${ }^{\text {b }}$ B.I. Kim ${ }^{\text {a }}$

drkbi@yuhs.ac

aDepartment of Preventive Dentistry and Public Oral Health, BK 21 PLUS Project, Yonsei University College of Dentistry, Seoul, Republic of Korea; ${ }^{b}$ Department of Preventive Dentistry and Public Oral Health, Yonsei University College of Dentistry, Seoul, Republic of Korea; ' Department of Oral and Maxillofacial Radiology, Yonsei University College of Dentistry, Seoul, Republic of Korea

The QLF Score for Occlusal caries (QS-Occlusal) reflecting fluorescence properties of non-cavitated occlusal caries. The score was divided into four levels in order to assess the non-cavitated occlusal caries intuitively. The aims of this study were to determine the validity and reliability of QS-Occlusal and to evaluate its use as a clinical criterion in occlusal caries detection. One hundred permanent premolar and molar teeth (ICDAS code $0-4$ ) were selected and QLF images were taken using Quantitative Light-induced Fluorescence-Digital. Three examiners assessed the QLF images using QS-Occlusal $(0=$ sound, $1=$ fluorescence changes within pits and fissures, 2 = extension of fluorescence changes, 3 = red fluorescence glow and dark shadow from dentin). Histological characteristics were revealed from polarized light microscopy images and they were scored according to the lesion severity $(0=$ sound, $1=$ enamel outer half, 2 = enamel inner half, 3 = dentin). Validity of QS-Occlusal was calculated at three thresholds (D1, histological score 0 vs. 1-3; D2, 0-1 vs. 2-3; D3, 0-2 vs. 3). Additionally, interand intra-examiner agreements were assessed using the intra-class correlation coefficient (ICC). There was a high correlation observed between histological evaluation and QS-Occlusal assessment results $(\mathrm{r}=0.78 ; \mathrm{p}<0.001)$. At the D1 threshold, QS-Occlusal had high sensitivity (0.95) with AUROC of 0.92 [95\% CI $=0.87-$ 0.98 ] and moderate specificity (0.67). At the D2 threshold, AUROC was 0.87 [0.81-0.94] with good validity (sensitivity $=0.87$, specificity $=0.77)$. At the D3 threshold, QS-Occlusal also exhibited excellent sensitivity (0.83), specificity (0.90), and AUROC of 0.90 [0.83$0.98]$. The intra- and inter-examiner agreements showed high reliability with ICC values of $0.85-0.97$ and $0.86-0.91$, respectively. In conclusion, the QS-Occlusal reflecting tooth fluorescence properties may be used as an evaluation criterion for assessing non-cavitated lesions with an excellent validity and reliability.

This work was supported by Basic Science Research Program through the National Research Foundation of Korea (NRF) funded by the Ministry of Education under grant (2016R1D1A1A09916934). 
159

\section{Residual Volumes After Cavity Preparation of Teeth Classified According to ICDAS II}

F. Yanikoglu*a, C. Cetinbay ${ }^{\mathrm{b}}$, K. Erkan $^{\mathrm{b}}$, B. Korkut $^{\mathrm{a}}$, Z.C. Pehlivan-Celik ${ }^{\text {a }}$ A. Kocaman', D. Tagtekin ${ }^{\text {a }}$

fyanikoglu@marmara.edu.tr

aDepartment of Restorative Dentistry, Faculty of Dentistry of Marmara University, Istanbul, Turkey; ${ }^{\mathrm{b}}$ Faculty of Dentistry of Marmara University, Istanbul, Turkey; ' Department of Metalurgy and Material Engineering, Faculty of Engineering, Istanbul Technical University, Istanbul, Turkey

The International Caries Detection and Assessment System (ICDAS II) is a clinical scoring method which defines the stage of caries lesions. ICDAS 3,4 and 5 are the scores of teeth which need to be restored mostly by direct approach. This study aimed to determine the relationship between ICDAS II scores and volumetric tissue loss of teeth after cavities were prepared by the adhesive principle. A total of 42 extracted premolar teeth with ICDAS 3 ( $\mathrm{n}=$ $11)$, ICDAS $4(n=14)$, ICDAS $5(n=17)$ were included to study after informed consent taken from patients before extraction. Digital photographs and radiographic images were taken following disinfection and polishing procedures of all teeth. A single experienced examiner diagnosed all teeth in day light with naked eye by using ICDAS criteria. Cavities were prepared using the adhesive principle and scanned by CAD/CAM (CEREC Omnicam, Dentsply-Sirona). Coronal volumes were measured after cavities were built-up using wax. The volumes of these $3 \mathrm{D}$ images were calculated in Meshmixer 3.3. Data were analyzed by using regression and correlation analysis. The mean volume losses were: ICDAS $3=$ $12.3 \%$ and $41.5 \mathrm{~mm}^{3}$, ICDAS $4=14 \%$ and $67.9 \mathrm{~mm}^{3}$ and for ICDAS $5=30.4 \%$ and $121.2 \mathrm{~mm}^{3}$. There was a significant positive relationship between ICDAS II scores and the volumetric tissue loss $(\mathrm{r}(42)=0.68, \mathrm{p}<.0001)$. Lowest standard deviation (LSD) was detected in ICDAS 4 teeth $(\mathrm{SD}=0.034)$ which indicates similar cavity dimensions after preparation. The greater variation in the other ICDAS classes may be due to the clinical difficulties in preparing standard cavities in these extensive lesions. In conclusion, caries assessments according to ICDAS scores were proportional to volumetic dental tissue losses.

We would like to thank to Cigdem Kumarki and DentsplySirona for their helps.

\section{0}

Soprolife ${ }^{\circledR}$ Camera: Agreement Among Examiners for Carious Lesion Activity Assessment

S. Doméjean*a,b ${ }^{*}$ N. Drancourt ${ }^{\mathrm{a}, \mathrm{b}}$, B. Pereira ${ }^{\mathrm{c}}$, V. Roger-Leroi ${ }^{\mathrm{a}, \mathrm{b}}$

sophie.domejean@uca.fr

aUFR d'Odontologie de Clermont-Ferrand, Centre de

Recherche en Odontologie Clinique EA 4847, Université

Clermont Auvergne, Clermont-Ferrand, France; ${ }^{\mathrm{b}} \mathrm{CHU}$ Estaing

Clermont-Ferrand, Service d'Odontologie, F-63000 Clermont-

Ferrand, France; ' ${ }^{C} \mathrm{CHU}$ Clermont-Ferrand, Direction de la

Recherche Clinique et des Innovations, F-63000 Clermont-

Ferrand, France

It is now commonly accepted that caries diagnosis at the lesion level encompasses, beside the detection (presence/absence) and the severity assessment (depth and cavitation), the activity assessment (active/inactive). Several concepts/tools have been described in the literature and/or by manufacturers for the lesion activity assessment (LAA): systems based on visual and tactile criteria, devices based on $\mathrm{pH}$ assessment or based on optics/light such as the Soprolife ${ }^{\circledR}$ camera. This study aims to investigate, ex-vivo, the agreement, inter- and intra-examiner, related to LAA using the Soprolife ${ }^{\circledR}$ camera. Pictures of 121 occlusal surfaces of permanent molars were captured, ex-vivo, using the Soprolife ${ }^{\circledR}$ camera (detection mode) within 48 hours after extraction. The pictures were shown twice (T0 and T0+15 days) to 3 examiners (Ex1, Ex2, Ex3) who were graduated dentists trained to the manufacturer recommendations for LAA (active/inactive according to the fluorescence color). The 3 examiners were asked to record independently the activity status of each of the 121 occlusal surfaces. Statistical analysis (Cohen Kappa; 95\% confidence interval (CI)) was run using Stata ${ }^{\circledR}$. Soprolife ${ }^{\circledR}$ camera showed moderate to substantial agreements between examiners with Kappa values from 0.48 [0.34-0.60] (Ex1 versus Ex2 at T0) to 0.70 [0.57-0.83] (Ex1 versus Ex3 at T0+15 days). Similar agreements (moderate to substantial) were found for the intra-examiner reproducibility: from 0.51 [0.36-0.67] (Ex3) to 0.74 [0.620.86] (Ex1). This survey, the first of its kind, showed that Soprolife ${ }^{\circledR}$ camera may be a promising tool for LAA in clinical practice. Nevertheless, further investigations are needed to fully validate its use.

\section{1 \\ OCT Imaging of Carious Lesions and Dental Structures - Center Wavelengths $1310 \mathrm{~nm}$ vs. $1550 \mathrm{~nm}$ \\ H. Schneider*a, M. Pues ${ }^{\mathrm{b}}$, C. Rüger ${ }^{\mathrm{a}}$, R. Haak ${ }^{\mathrm{a}}$ \\ hartmut.schneider@medizin.uni-leipzig.de \\ aDepartment of Cariology, Endodontology and \\ Periodontology, University of Leipzig, Leipzig, Germany; \\ 'Thorlabs GmbH, Lübeck, Germany}

Light scattering and absorption are determining factors in the imaging of intraoral hard and soft tissues using optical coherence tomography (OCT). The performance of OCT in the approaches 
spectral domain (SD-OCT) or swept source (SS-OCT) with center wavelengths of $1310 \mathrm{~nm}$ and $1550 \mathrm{~nm}$ were assessed for the detection of carious lesions and dental hard tissue structures. 17 extracted carious human teeth were visually selected (ICDAS-II code 0 -2). Three of that were restored with composite or GIZ (Class I, $\mathrm{V})$. One region of interest (ROI) of each tooth was marked and imaged using SD-OCT (Telesto-II; $1310 \pm 107 \mathrm{~nm}$, power on sample $3 \mathrm{~mW}$ ), SD-OCT (protoype, $1550 \pm 110 \mathrm{~nm}, 4.5 \mathrm{~mW}$ ) and SS-OCT (VEGA110; $1310 \pm 50 \mathrm{~nm}, 10 \mathrm{~mW}$ ) (all Thorlabs GmbH, Dachau, G). Two calibrated examiners classified the "penetration depth" (PD) and "spatial resolution" (SR) at 17 ROI into the following categories: 1 (excellent), 2 (good), 3 (satisfactory). OCT signals were validated using light microscopy. The examiners and the OCT systems were statistically compared (Wilcoxon-test, $\alpha=$ $0.05)$. In vivo two restored premolars (composite, Class $\mathrm{V}$ ) and two incisors (ICDAS-II code 2) were imaged using SD-OCT. 1) For both parameters the ratings of examiners were not significantly different (p: 0.125-0.707). 2) The PD increased up to 93\% (in vitro/ in vivo). $\mathrm{PD}$ values were rated significantly higher using $1550-\mathrm{SD}$ compared to $1310-\mathrm{SD}$ ( $\mathrm{p}: \leq 0.003$, in vitro) or with enhanced power of the 1310-SS vs. 1310-SD $(<0.001)$. No significant difference was seen between 1310 -SS vs. $1500-S D(\geq 0.682)$. 3) The SR was rated higher using 1310-SD compared to $1550-\mathrm{SD}(\leq 0.034)$ or 1310 -SS $(<0.001)$, but not with 1310 -SS vs. 1550 -SD $(\geq 0.170)$. SDOCT centered at $1550 \mathrm{~nm}$ enables deep and satisfactory resolved images of dental hard and soft tissues also with lower power on sample.

Supported: EFRE/SMWK (OCT-100175024).

\section{2 \\ Effects of Different Light Sources on QLF Analysis of Caries-Like Lesions In Vitro}

E.A. Zawia, M. Malinowski*, S.M. Strafford, K.J. Toumba

M.Malinowski@leeds.ac.uk

Department of Paediatric Dentistry, School of Dentistry, University of Leeds, Leeds, UK

The aim was to assess of the effect of different light sources on Quantitative Light-Induced Fluorescence (QLF) analysis during demineralisation and remineralisation of enamel caries-like subsurface lesions in vitro. Enamel slabs $(n=60)$ with artificial carieslike lesions were randomly allocated to two treatment groups 1) $1450 \mathrm{ppm} F$ toothpaste group and 2) $0 \mathrm{ppm} \mathrm{F}$ toothpaste control group. Each slab was analysed using QLF under dark conditions (control) (3-lux), office lighting (20-lux), portable lamp light (540lux) and dental chair lighting (680-lux). For each light source the following parameters: $\Delta \mathrm{F}, \Delta \mathrm{Q}$ and area were recorded at baseline and after 28 days of a $\mathrm{pH}$ cycling regime. Wilcoxon test showed significant $(\mathrm{p}<0.001)$ differences in the parameters were recorded between the four light groups at baseline and after treatment. However, significant differences $(\mathrm{p}<0.001)$ between $\Delta \mathrm{F}$ and the four light conditions, Following $\mathrm{pH}$ cycling, the differences in all parameters were significant $(\mathrm{p}<0.05)$ between: fluoride-free and $1450 \mathrm{ppm} F$ toothpastes for all light sources (Mann Whitney test). Values recorded using dental chair lighting (brightest) showed a significant $(\mathrm{p}<0.001)$ underestimation of lesion size compared to the values obtained with the under dark conditions (Wilcoxon test). In conclusion, the differences in QLF parameters recorded under different light sources at baseline and after fluoride treatment were statistically significant when compared to the values recorded under dark conditions. The differences before and after treatment as well as the differences between the treatment and control groups could be detected under standardised light conditions. Dental chair lighting should not be used for QLF assessment of early enamel lesions, as there is wide variation and inconsistency with dental chair lighting.

\section{3 \\ Optical Magnification in Detecting Occlusal Caries Lesions in Permanent Molars Using Visual Scoring Systems}

M.B. Diniz ${ }^{* a}$, S. Wilde ${ }^{\text {b }, ~ P . H . ~ C a m p o s ~}{ }^{\mathrm{a}}$, A.P.M. Marcondes ${ }^{\mathrm{a}}$, C. Moura-Nettoc, T.F. Novaes ${ }^{\mathrm{a}}$, A. Lussid

mibdiniz@hotmail.com

aSchool of Dentistry, Cruzeiro do Sul University, São PauloSP, Brazil; ' School of Dentistry, Rio Grande do Sul Federal University, Porto Alegre-RS, Brazil; 'School of Dentistry, Paulista University, São Paulo-SP, Brazil; ${ }^{d}$ Department of Preventive, Restorative and Pediatric Dentistry, School of Dental Medicine, University of Bern, Bern, Switzerland

The aim of this study was to evaluate, in vitro, the influence of different levels of optical magnification in detecting occlusal caries lesions in permanent molars using three visual scoring systems. One occlusal site per tooth was analyzed in 120 freshly extracted human permanent molars. Two trained examiners inspected the teeth using the ICDAS (International Caries Detection and Assessment System), CAST (Caries Assessment Spectrum and Treatment), and ADA-CCS (American Dental Association-Caries Classification System) criteria. The study was conducted in three methods: (A) without optical magnification, (B) using a binocular lens with $3.5 \times$ magnification, and (C) using an operating microscope with $16 \times$ magnification. During each phase, the teeth were evaluated twice using each visual scoring system, with a one-week interval between examinations. Then, the teeth were sectioned for histological analysis. Kappa values for intra- and inter-examiner reproducibility were good to excellent for all three visual scoring systems at each study phase. Histological results showed that seven sites $(5.8 \%)$ had a score of $0,28(23.3 \%)$ had a score of $1,43(35.9 \%)$ had a score of $2,18(15.0 \%)$ had a score of 3 , and $24(20.0 \%)$ had a score of 4 . For the diagnostic $\mathrm{D}_{1}$ threshold (lesions in enamel and dentine), sensitivity (0.86-0.95), accuracy (0.86-0.93), and area under the ROC curve (Az) (0.754-0.865) were high for each scoring system in all study phases. CAST presented statistically higher specificity values (0.64-0.82) compared to the other scoring system $(\mathrm{p}<0.05)$. Decreased specificity was observed with ICDAS (0.43), CAST (0.64), and ADA-CCS (0.39) when evaluating with the operating microscope $(\mathrm{p}<0.05)$. For the diagnostic $\mathrm{D}_{3}$ threshold (dentine lesions), there was no statistically significant difference between the visual scoring systems during the three study methods $(p>0.05)$. It can be concluded that magnification negatively influenced the visual detection of occlusal caries lesions in 
permanent molars, overestimating the number of those lesions. The use of ICDAS, CAST and ADA-CCS criteria provided similar results without magnification or using a binocular lens with $3.5 \times$ magnification.

Funded by Coordenação de Aperfeiçoamento de Pessoal de Nível Superior - CAPES.

\section{4 \\ Reproducibility of Fluorescence-Based Cameras with and without Autofocus for Detection of Caries in Primary Teeth}

\author{
A. Jablonski-Momeni*a ${ }^{\text {, H. Korbmacher-Steiner }}{ }^{\mathrm{a}}$, D. Kappert ${ }^{\mathrm{b}}$ \\ momeni@staff.uni-marburg.de \\ ${ }^{a}$ Department of Orthodontics, Philipps-University Marburg,

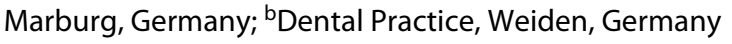

Fluorescence-based intraoral camera systems can be used as a supportive tool for caries detection. This study aimed to evaluate the reproducibility of two fluorescence-based cameras for detection of caries in primary teeth: The newly introduced VistaCam iX HD (FC1) with an autofocus system and HD image quality, and the VistaCam iX (FC2, without autofocus). Furthermore, measurements between FC1 and FC2 should be compared. Investigation sites on 55 extracted primary teeth were cleaned and assessed visually (ICDAS). Two examiners (A, B) performed fluorescence measurements independently, using FC1 and FC2. All sites were re-assessed after five days. Statistical analyses were performed with MedCalc (Version 16.8.4). Correlation between the methods was analyzed using Spearman's rank correlation coefficient (rs). Intraand inter-examiner reproducibility values were calculated using the intraclass correlation coefficient (ICC). Wilcoxon-test was used to compare the readings between FC1 and FC2 $(\alpha=0.05)$. Significant correlation was found between visual and fluorescence findings $(\mathrm{p}<0.001)$ : FC1 (A/B): rs = 0.655/0.675; FC2 (A/B): rs = $0.431 / 0.626$. ICC for intra-examiner reproducibility were: $\mathrm{FC} 1$ $(\mathrm{A} / \mathrm{B})=0.957 / 0.986$ and $\mathrm{FC} 2(\mathrm{~A} / \mathrm{B})=0.883 / 0.787$, respectively. ICC for inter-examiner reproducibility were: $\mathrm{FC} 1=0.961$ and FC2 $=0.786$, respectively. Significant differences were calculated between the readings of FC1 and FC2 for both examiners ( $\mathrm{p}<$ 0.0001 ). Intra- and inter-reproducibility values of each fluorescence-based camera were in a high range. Nevertheless, significant differences between the performance of the camera systems were found for the detection of caries in-vitro.

Acknowledgement: The intraoral camera systems were provided by Dürr Dental, Bietigheim-Bissingen, Germany.

\section{5 \\ Correlation Between the DMFT Score of the 12-Years Old Children and the Concentration of Fluoride in Drinking Water from Macedonia}

\author{
V. Ambarkova*a, M. Jankulovska ${ }^{\mathrm{a}}$, M. Kochubovski ${ }^{\mathrm{b}}$, \\ E. Gjorgievska ${ }^{\mathrm{a}}$, E. Zabokova-Bilbilova ${ }^{\mathrm{a}}$, A. Sotirovska-lvkovska ${ }^{\mathrm{a}}$, \\ N. Stavrevac
}

vesna.ambarkova@gmail.com

aDepartment of Preventive and Pediatric Dentistry, Faculty of Dentistry, University Ss Cyril \& Methodius-Skopje, Republic of Macedonia; ${ }^{\text {b}}$ The Institute of Public Health in the Republic of Macedonia; 'Department of Fixed Prosthodontics, Faculty of Dentistry, University Ss Cyril \& Methodius-Skopje, Republic of Macedonia

The aim of the study was to determine the correlation between the DMFT score of 12-years old children and the concentration of fluorine in drinking water from the settlements from the Eastern region of the Republic of Macedonia. This research involved 396 students from 9 Central and 13 Regional Primary Schools and the DMFT score was recorded. The students live in four different cities and fifteen different villages. The nineteen water samples were taken in the study area to determine their fluoride concentration by electrochemical method using a special ion Analyser ( $\mathrm{pH} / \mathrm{ISE}$ meter-Thermo-Orion) with a special F-electrode (Thermo Orion Ion Plus Fluoride Electrode) at the Institute of Public Health. The Spearman Rank Order Correlations was used for statistical analysis. Results The total number of children in the sample was 396, comprising 201 (50.8\%) females and 195 (49.2\%) males. The mean DMFT was 3.47, with standard deviation (SD) of 2.9. The maximum concentration of fluorine was determined in drinking water from suburban settlement Tri Češmi and Štip city $(0.47 \mathrm{ppmF})$ and the minimum concentration $(0.26 \mathrm{ppmF})$ from Sveti Nikole city and $(0.08 \mathrm{ppmF})$ from Šašavarlija village. The correlation between the value of the DMFT score of the 12 years old children from the Eastern region, and the concentration of fluoride in drinking water was negative, indirect with the value of the coefficient $r=-0.03$. The correlation between the value of the DMFT score and the concentration of fluoride in drinking water was not significant probably due to the sub-optimal F concentrations in all water samples investigated. 


\section{6 \\ Where Do the US Pediatric Dental Practitioners Stand regarding Molar Incisor Hypomineralization (MIH)?}

\author{
A. Tagelsir*a, J.A. Dean ${ }^{\text {b }}$, G.J. Eckert ${ }^{\mathrm{c}}$, A.E. Martinez Mier ${ }^{\mathrm{a}}$ \\ azzahmed@iupui.edu \\ aDepartment of Cariology, Operative Dentistry and Dental \\ Public Health, Indiana University School of Dentistry,

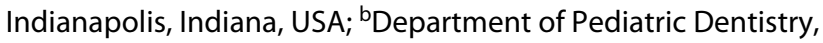 \\ Indiana University School of Dentistry, Indianapolis, Indiana, \\ USA; 'Department of Biostatistics, Indiana University School of \\ Medicine, Indianapolis, Indiana, USA
}

With the lack of data from the US investigating dentists' level of perception regarding Molar Incisor Hypomineralization (MIH), this survey-based study targeted US pediatric dentists to determine their knowledge, perception and clinical management strategies of MIH. After obtaining AAPD and IRB authorizations, pediatric dentists identified from the AAPD's 2016-2017 membership directory in the Midwest were sent email invitations to participate in the study. The questionnaire, adapted from pervious studies, had two main sections; demographics, educational and clinical practice information and $\mathrm{MIH}$-focused questions including knowledge and perceptions of MIH's estimated prevalence, diagnosis, clinical challenges and restorative management options. Descriptive statistics and Chi-square tests were used for analysis. Alpha level < 0.05 was considered statistically significant. A total of 251 out of 975 surveys were completed (response rate $26 \%$ ). Nearly all participants were familiar with MIH. Most respondents observed $\mathrm{MIH}$ in either less than $10 \%$ or around $10-25 \%$ in their clinical practice (62\% and 35\%, respectively). The majority were aware of the lack of MIH prevalence data from the USA and believed that $\mathrm{MIH}$ is a significant clinical problem that demands further investigation ( $90 \%$ and $85 \%$, respectively). Most respondents were very confident (65\%) or confident (34\%) in diagnosing teeth with MIH. The most cited clinical challenge in managing MIH teeth were "long-term success of restorations" (79\%). Stainless steel crowns and composite resins were the most used dental material by respondents (32\% and 29\%, respectively). When analyzed individually, perceived MIH prevalence, diagnosis, clinical challenges, and restorative options differed significantly per certain demographics, educational and clinical practice characteristics $(\mathrm{p}<0.05)$. $\mathrm{MIH}$ is generally well acknowledged by pediatric dentists in the US Midwest, with some discrepancy related to its diagnosis, management challenges, and restorative options.

The study received no external funding.

\section{7 \\ Tooth-Coloured GICs Containing Discrete Silver Nanoparticles for Lasting Prevention Against Microbial Biofilm Formation}

G.C. Cotton ${ }^{\mathrm{a}, \mathrm{b}}$, D. Siddhantac, D.R. Schwass*b,c, G. Tompkins ${ }^{\mathrm{b}}$, C.J. Meledandri ${ }^{\mathrm{a}, \mathrm{b}}$

aDepartment of Chemistry, University of Otago, Otago, New Zealand; 'bir John Walsh Research Institute, University of Otago, Otago, New Zealand; 'Faculty of Dentistry, University of Otago, Otago, New Zealand

Toward the goal of creating an effective strategy for the prevention of secondary caries we have developed silver nanoparticlemodified GICs (AgNP-modified GICs), with an established antibiofilm surface effect and a restoration colour consistent with tooth shade guides. Monoculture biofilms of Streptococcus mutans, S. mitis, S. sanguinis, and Psuedomonas aeruginosa were cultured (72 h) in brain heart infusion broth (1\% sucrose) on AgNP-modified $(6.4 \mu \mathrm{g} \mathrm{Ag})$ and non-modified GC Fuji IX GIC specimens $(n=3)$. The biofilm/GIC specimens were treated with Live/dead ${ }^{\circledR} \mathrm{Ba}$ clight $^{\mathrm{TM}}$. Stained biofilms were examined with a Zeiss LSM 710 confocal laser-scanning microscope. Biofilm/GIC specimens were also observed using scanning electron microscopy. Additionally, S. mutans biofilms were cultured ( $72 \mathrm{~h}$ ) on GIC discs at weekly intervals between artificial aging of the GICs in MQ H2O. Flexural strength testing was performed according to ISO protocol 9917:2:2010 ( $\mathrm{n}=35)$. Significant biofilm reduction on AgNPmodified Fuji IX GICs of greater than $90 \%$ was observed in both CLSM, and SEM imaging, when compared to non-modified Fuji IX GICs. Flexural strength testing of AgNP-modified GICs demonstrated increased strength values with AgNP incorporation (17.44 MPa, $\mathrm{p}=0.0149)$ compared to the Fuji IX control GIC (14.6 $\mathrm{MPa}$ ). Comparison of AgNP-modified GICs to the Vita shade guide indicated tooth shades consistent with the material (i.e. A3.5 to $\mathrm{C} 4$ dependent on AgNP concentration in material). The incorporation of AgNPs within GICs may aid the reduction of secondary caries due to the prevention of biofilm growth at the GIC surface. The AgNPs have been observed to provide the GICs with an antibiofilm effect over a period of at least 3 months. AgNP-modified GICs demonstrate increased flexural strength when compared to the non-modified Fuji IX GIC and were consistent with tooth shade colouration.

This work was funded through Silventum Ltd., Otago Innovation Ltd. and supported through a University of Otago PhD scholarship. 
168

MIH Prevalence in 12-Year-Old Children in Germany

U. Schiffner*a, R. Jordan ${ }^{b}$

schiffner@uke.uni-hamburg.de

aDepartment of Conservative and Preventive Dentistry,

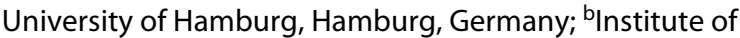
German Dentists, Cologne, Germany

Molar-Incisor-Hypomineralisation ( $\mathrm{MIH})$ is increasingly attracting attention from patients, parents and researchers. Thus, the aim of the evaluation was to determine MIH prevalence and severity in 12-year-old children in a representative sample in Germany. The data collection was part of the nationwide Fifth German Oral Health Study (DMS V) which was conducted in 2014. In this epidemiologic survey, 1,468 12-year-old children were examined for MIH and caries on first permanent molars and incisors by calibrated examiners. MIH was scored in accordance with the criteria of the European Academy of Paediatric Dentistry (EAPD) as opacity and minor or major enamel breakdown, atypical restoration or $\mathrm{MIH}$-caused extraction. The statistical evaluation included the evaluation of relations between MIH prevalence and gender, social class (education level of the parents), and caries. $28.7 \%$ of the children exhibited at least one MIH tooth. The prevalence of the most severe MIH condition was $23.3 \%$ for opacities and $5.4 \%$ for enamel breakdown (4.9\% minor breakdown or atypical restorations, $0.5 \%$ major breakdown or extraction). There was no gender nor social class related difference in $\mathrm{MIH}$ prevalence ( $\mathrm{p}=0.453$ and 0.607 , resp., $\mathrm{X}^{2}$-test). The caries prevalence was $18.7 \%$. There was a statistical relation between MIH prevalence and caries: In caries free children the MIH prevalence was $27.0 \%$, while it was $36.5 \%$ in children with caries $(p=0.002)$. Although the severity of most MIH defects was low, it has to be noted that the overall $\mathrm{MIH}$ prevalence is higher than the caries prevalence. In particular, the percentage of $5.4 \%$ of the 12 -year-olds with at least one tooth exhibiting MIH-mediated enamel breakdown requires attention. 


\section{Author Index}

Abdallaoui, F. 78

Abouqal, R. 78

Abrahamsson, T. 29

Abreu Placeres, N. 91

Aimee, N.R. 55

Aimée, N.R. 59

Al Dehailan, L. 129

Al-Ahmad, A. 30

Al-Ani, A. 72, 73, 74, 75, 92

Alev-Tuzuner, B. 114

Alfaro, L. 121

Algarni, A.A. 2

Alkilzy, M. 73, 74, 92, 125

Altenburger, M.J. 30

Alvarado, A.B. 108

Alves, L.S. 86, 87

Ambarkova, V. 11, 79, 151, 165

Americano, G.C.A. 77

Anandakrishna, L. 82

Anderson, A.C. 30

Anderson, P. 14, 150

Ando, M. 156

Aragão, M.G.B. 108

Arango-De la Cruz, M.C. 121

Aranha, A.C.C. 7

Araujo, M.P. 57

Aresti, M. 26

Argi, Q. 17

Arrica, M. 123

Arruda, I.S. 127,128

Artacho, A. 29

Arthur, R.A. 21

Ascenso, C. 17

Assunção, C.M. 4

Atkinson, G. 115

Avila, V. 66

Ávila, V. 91

Azevedo, C.B. 58

Bælum, V. 37

Baghlaf, K. 70

Baginska, J. 84, 88

Bagnato, V.S. 25

Bahal, P. 155

Bakhshandeh, A. 133

Bakshandeh, A. 136

Bandeira, A.V.L. 108

Basner, R. 72

Basta, A. 72, 75

Basu, N. 102
Baumann, T. 7, 8, 9, 10

Baysan, A. 96, 150

Beighton, D. 22, 31

Benetti, A.R. 3

Benito, A. 102

Berg, T. 48

Bernardino, B.A. 116

Beyeler, B. 10

Bezerra, D.S. 34

Bezerra, G.L. 127, 128

Birk, L. 142

Bittencourt, P.F. 55

Bjørndal, L. 52, 133, 135, 141

Boezewinkel, M. 148

Boix-Garcia, A. 117

Bonifacio, C.C. 118

Bonifácio, C.C. 57, 120

Bontà, G. 130, 149

Bortone, A. 40

Borum, M. 133

Boteva, E. 49

Bottenberg, P. 81, 97

Bour, A. 78

Bradshaw, D.J. 18

Braga, M.M. 47, 116, 138, 140

Brown, C. 36

Brusius, C.D. 86,87

Buchalla, W. 110, 113

Buckley, C. 102, 129

Buenger, L. 48

Burnside, G. 70

Buzalaf, M. 103

Cabrera, D.F. 66

Caddeo, C. 40

Cagetti, M.G. 123, 126, 130, 149

Camoni, N. 126

Campos, P.H. 163

Campus, G. 123, 126, 130, 149

Cankar, K. 132, 137, 142

Cantoral, A. 102

Capobianco, G. 123

Capocci, M. 61

Caraballo, M.G.D. 146

CARDEC Collaborative Group 47

Cardoso, A.C. 31

Carmo, J. 17

Carpenter, G. 150

Carpenter, L. 33

Cartagena, A. 136
Carvalho, J.C. 55, 59

Carvalho, P. 17

Carvalho, T.S. 7, 8, 9, 10

Casagrande, L. 58

Cassiano, L. 146

Castelo-Branco, C.M. 116

Castiblanco, G.A. 66, 102, 129

Catmull, D. 33

Cetinbay, C. 159

Chałas, R. 41

Chagas, F.O. 127, 128

Chala, S. 78

Chang, J. 157

Chapman, N. 155

Chaubron, F. 145

Chen, J. 23

Cheng, L. 23

Cheraqi, T. 143

Choi, Y.H. 131

Chung, M.J. 158

Chung, S.H. 157

Cieplik, F. 110

Cifuentes-Aguirre, O.L. 121

Cloitre, T. 153

Cocco, F. 61, 123, 126, 130, 149

Coelho, C.S.S. 99, 108

Collado, M.C. 29

Columbano, M. 123

Conceição, J.P. 54

Corridore, D. 61

Cortes, A. 121

Cortés, A. 66

Costa-Oliveira, B.E. 111

Cotton, G.C. 167

Coulot, C. 53

Crielaard, W. 35

Crombie, F. 134

Cuisinier, F. 145, 153

Curnow, M. 70

Cury, J.A. 27, 98, 99, 100, 101, 107, 108, 111

da Mata, C. 122

Dame-Teixeira, N. 32

Damé-Teixeira, N. 22, 31

Dashper, S.G. 33

Davi, J. 123

de Aguiar, M.H.R. 127, 128

de Andrade, C.G. 21

de Assis, A.C.M. 111 
de Jong, E.de.J. 24

de Josselin de Jong, E. 19, 65

de Oliveira, K.T. 25

de Soet, J.J. 90

de Souza Rastelli, A.N. 25

Dean, J.A. 166

Deery, C. 138

Del Bel Cury, A.A. 27, 101, 108

Delgado, A.J. 146

Demant, S. 141

Demarchi, K.M. 45

Deng, D.M. 23, 25, 35

Desoutter, A. 145

Dessole, S. 123

Devine, D.A. 22, 31, 32

D'hallewin, G. 40

Díaz-Garrido, N. 109

Dige, I. 28, 37

Dilbone, D.A. 146

Diniz, M.B. 163

Do, T. 22, 31, 32

Dolenec, E. 80

Doméjean, S. 53, 160

Döpp, S. 90

Drake, P. 155

Drancourt, N. 160

Duerrschnabel, A. 5, 6

Durward, C. 134

Dzidic, M. 29, 117

Ebensberger, H. 113

Eckert, G.E. 2

Eckert, G.J. 102, 103, 129, 156, 166

Eidt, G. 21

Ekstrand, E.K. 138

Ekstrand, K.R. 3, 66, 89, 91, 136, 140

Enax, J. 110

Erkan, K. 159

Esteves-Oliveira, M. 16, 43, 154

Ev, L.D. 22

Fechine, F.V. 127, 128

Federlin, M. 113

Fernandez, C.E. 101

Fernando, J.R. 20

Ferreira, R.S. 12

Ferrer, M. 119

Ferrer, M.D. 117

Ferstl, G. 113

Finderle, Z. 132

Floriano, I. 47, 54, 136, 138

Fonseca, S.G.C. 127, 128

Fonteles, M.M.F. 127, 128

Formann, B. 125

Fortich-Mesa, N. 121

Fransson, H. 133

Frasconi, G. 123

Furrer, S. 8, 9

Furtado Jr, J.H.C. 127, 128
Gaard, H. 133

Gamboa, L.F. 66, 93, 121

Ganss, C. 1, 42, 62, 64

Garcia, E. 52, 135

Garcia, J.E. 38

Ge, Y. 23

Georgiev, Z. 151

Geraldeli, S. 146

Gergely, C. 153

Giacaman, R.A. 109

Giese-Kraft, K. 42

Gimenez, T. 54, 138

Gjorgievska, E. 165

Glökler, J. 43

Gomes, R.A. 47

Gonçalves, G.S.A. 77

González-Cabezas, C. 2

Göstemeyer, G. 122, 124

Gouvêa, D.B. 58

Groselj, M. 132, 137, 142

Guedes, S.F.F. 34

Guerra, F. 61, 63

Guimarães, T.T. 77

Güner Onur, Ş. 139

Gussy, M. 33

Haak, R. 48, 95, 161

Hamama, H.H. 46

Hara, A.T. 2

Harth-Chúc, E. 34

Hassanali, L. 14

Hayran, O. 114

Haznedaroğlu, E. 139

He, T. 155

Heins, M. 64

Hellwig, E. 30

Herlofson, B.B. 60

Hernandez-Avila, M. 102

Hesse, D. 57, 120

Higham, S.M. 18, 36

Hilgert, L.A. 45

Hill, R. 96

Hiller, K.-A. 110, 113

Hirsch, C. 95

Homayouni, A. 60

Hope, C.K. 36

Hove, L.H. 60

Høvenhoff, L. 133

Hu, H. 102

Imparato, J.C. 116

Innes, N.P. 57

Iordanova, G. 49

Italian study group on ECC 123

Jablonski-Momeni, A. 164

Jacquet, W. 81

Jamiolkowski, J. 84, 88

Jan, J. 80

Jankulovska, M. 165
Jansen, P. 154

Jenmalm, M.C. 29

Jensen, J.L. 60

João-Souza, S.H. 7

Jones, S. 155

Jordan, R. 168

Juliano, C. 40

Jung, E.H. 158

Kahharova, D.A. 65

Kahtani, A.L. 150

Kaminska, I. 84, 88

Kaminski, K. 84, 88

Kamp, A. 38

Kang, S.M. 24

Kappert, D. 164

Kapusevska, B. 79

Kara, E. 110

Kargul, B. 13

Karpukhina, N. 96

Karygianni, L. 30

Kato, K. 106

Kemnitz, E. 16

Khudanov, B. 97

Khudanov, B.O. 65

Kierklo, A. 84

Kiess, W. 95

Kim, A.O. 112

Kim, B.I. 19, 24, 65, 158

Kim, H.J. 112

Kim, S.K. 19

Kocaman, A. 159

Kochubovski, M. 165

Korbmacher-Steiner, H. 164

Korkut, B. 159

Kouzmina, E. 85

Kramer, G. 148

Krapež, J. 80

Krause, F. 48, 95

Kreuz, M. 95

Krohn, S. 48

Krois, J. 124

Kubik-Komar, A. 41

Kuzmina, I. 85, 89

Kuznetsov, P. 85

Kwon, H.K. 19, 158

Ladewig, N.M. 47

Lamers, M.L. 21

Lamme, J. 147

Lapinska, M. 88

Łapińska, M. 84

Lausch, J. 16

İldeş, G.Ç. 139

Le Cao, K.-A. 33

Leal, A.M.C. 108

Leal, F.L. 87

Leal, J.P. 12

Leal, S.C. 45, 52, 58, 69, 135

Lee, E.S. $19,24,65$ 
Lee, H.S. 19, 158

Levallois, B. 153

Lia, E.N. 69

Ligtenberg, A.J. 35

Lingström, P. 123, 126, 130, 149

Lippert, F. 2, 102, 129

Livesu, R. 126

Llena, C. 117, 119

Lobo, P.L.D. 127,128

Lock, N.C. 86

Löffler, M. 95

Lohinai, Z. 26

Lopez-Lopez, A. 117

López-López, A. 119

Lotfi, G. 143

Lotif, M.A.L. 127

Lourenço, J. 59

Luka, B. 5, 6

Lussi, A. 4, 7, 8, 9, 10, 163

Lynch, R.J. 14

Lynch, R.J.M. 18

Machado, G. 54

Machado, G.M. 47

Machado, J.A. 77

Maguire, A. 103, 115

Maioli, M. 40

Maisch, T. 110

Malinowski, M. 162

Maltz, M. 22, 31, 32, 86, 87

Manca, M. 40

Manconi, M. 40

Manso, A.G. 17

Manton, D.J. 134

Marcondes, A.P.M. 163

Marín, D.J. 93

Marques, L.A.S.P.F. 100

Marques, M.G. 45

Marro, F. 81

Martens, L. 81

Martignon, S. 66, 91, 92, 93, 121

Martinez Mier, A.E. 166

Martinez-Mier, A. 103

Martinez-Mier, E.A. 102

Martins Reis, A.C. 34

Maslak, E. 68

Masłowska, A. 84

Mastnak, M. 80

Matayoshi, S. 39

Matos, R. 140

Mazur, M. 63

McKenna, G. 122

Mejía, L. 121

Meledandri, C.J. 167

Mello-Moura, A.C.V. 54

Mendes, F.M. 47, 116, 120, 138, 140

Mendonça, J.F. 127, 128

Menteş, A. 139

Mercado-Garcia, A. 102

Mercer, J.B. 152
Mestrinho, H.D. 55, 59

Meyer-Lueckel, H. 16, 43, 154

Michou, S. 3

Midani, R. 67

Mijoska, A. 11, 56, 79, 151

Milia, E. 40

Mira, A. 29, 117, 119

Mitchell, H.L. 33

Moro, B.L.P. 140

Mouhat, M. 152

Mourad, M.S. 67, 72, 75, 92

Moura-Netto, C. 163

Muehler, D. 110

Muirhead, V. 70

Muller-Bolla, M. 53

Murbay, S. 46

Mustafa Ali, M. 67, 72, 75

Na, J.Y. 158

Nakano, K. 39

Nardi, G.M. 61, 63

Nascimento, M.M. 146

Naumova, E.A. 105

Ndokaj, A. 63

Negrini, T.C. 21

Nemeth, L. 137, 142

Neves, B.G. 34

Newton, J.T. 91

Nicolescu, T. 117, 119

Nie, M. 25

Nieddu, S. 123

Nikolovska, J. 56

Nobrega, D.F. 101

Nomura, R. 39

Novaes, T.F. 140, 163

Nyvad, B. 28, 51

Oglakcioglu, S. 50

Okutan, A.E. 139

Olegário, I.C. 57, 120

Örtengren, U. 152

Osokina, A. 68

Otsugu, M. 39

Ottenga, M.E. 146

Ottolenghi, L. 61, 63

Paes, A.B. 59

Pamir, T. 50

Panayotov, I. 145, 153

Park, H.R. 112

Park, K.J. 48

Park, K.-J. 95

Park, S.W. 19, 158

Park, T.J. 131

Parolo, C.C.F. 22, 31, 32

Paul, W. 36

Pehlivan-Celik, Z.C. 114,159

Peixoto, A. 17

Peker, S. 114

Pelissier, B. 145
Peng, X. 23

Pereira, B. 160

Pereira, P.N.R. 146

Pereira, R.A. 47

Perez-Vilaplana, S. 117

Peterson, K.E. 102

Petretto, G. 40

Pine, C. 70

Pinna, R. 40, 123

Pion, L.A. 116

Pitts, N. 91, 92

Pitts, N.B. 66

Platt, J.A. 2

Plett, W. 104

Poprawe, R. 43, 154

Pues, M. 161

Queiroz, A.C. 55

Rachana, S. 82

Raggio, D.P. 47, 54, 57, 116, 120, 138

Ramarao, S. 144

Ramos-Martínez, K. 121

Raposo, F. 69

Reda, S. 124

Reynolds, C. 20

Reynolds, E.C. 20, 33

Ribeiro, A.P. 146

Ribeiro, A.P.D. 45

Ricciardi, C. 149

Richards, A. 51

Ricketts, D.N. 138

Ricomini-Filho, A.P. 27, 107, 108, 111

Rinaldo, F. 61, 63

Roberts, J.M. 18

Rocha, E.S. 47

Rodakowska, E. 84,88

Rodrigues, A.C. 69

Rodrigues, J.A. 4, 58, 118

Rodrigues, L.K.A. 34

Roger-Leroi, V. 160

Rothballer, M. 30

Roulet, J.F. 146

Rüger, C. 161

Rupf, S. 1

Rusthen, S. 60

Rykke, M. 60

Rykwa, D. 41

Saeed, A. 150

Sah, O. 115

Said, D. 85,89

Sakae, L.O. 7

Salem, K. 143

Salobir, B. 80

Salvado, C. 17

Salvi, D. 63

Sampaio, A.A. 27

Sanchez, B. 102

Sanderson, R. 103 
Sanjuán-Acero, J.A. 121

Santamaria, R.M. 67, 92

Santos, N.M. 4, 58

Sarti, C.S. 58, 118

Sathyanarayanan, U. 144

Satta, L. 123

Scaramucci, T. 7

Scheerman, J.F.M. 147, 148

Schievelkamp, S. 16

Schiffner, U. 168

Schlafer, S. 37,38

Schlueter, N. 1, 5, 6, 42, 62, 64

Schmid, M. 30

Schmidt, J. 48, 95

Schmidt, P. 105

Schmidt, S.-C. 42,62

Schmoeckel, J. 67, 73, 74, 92

Schnarr, M. 1

Schneider, H. 48, 161

Scholz, K.J. 113

Schulte, A.G. 97, 104, 105

Schwass, D.R. 167

Schwendicke, F. 45, 122, 124

Sen Yavuz, B. 13

Sersa, I. 132

Sezgin, B.I. 139

Sharma, C.P. 36

Shen, P. 20

Shimazaki, Y. 106

Shwetha, G. 82

Siddhanta, D. 167

Silva, G.S.Q. 54

Silva, R.P. 4

Simoes, T.C. 127,128

Singh, P.B. 60

Sleibi, A. 96, 150

Slimani, A. 145, 153

Soares-Yoshikawa, A.L. 98

Solange, P. 33

Somi, L. 148

Sonda, N.C. 4

Sopharith, S. 134

Sotirovska Ivkovska, A. 11, 56, 79, 151

Sotirovska-Ivkovska, A. 165

Souza, S.E. 27

Soviero, V.M. 77

Splieth, C. 125

Splieth, C.H. 67, 72, 73, 74, 75, 92

Stachurska, Z. 84,88
Stangvaltaite, L. 133, 152

Staun Larsen, L. 51

Stavreva, N. 11, 56, 79, 151, 165

Steinberg, D. 26

Stensson, M. 29

Stollenwerk, J. 43, 154

Strafford, S.M. 162

Strittmatter, E. 5

Strohmenger, L. 130, 149

Sugiyama, S. 44

Susin, C. 86,87

Szpakowicz, M. 84, 88

Tabchoury, C.P.M. 12, 98, 99, 100, 107, 108

Tagelsir, A. 129, 166

Tagtekin, D. 15, 159

Takriti, M. 73, 74

Tamura, K. 106

Tang, D.Y. 71

Tappuni, A.R. 96

Tarcin, B. 114

Tardivo, D. 153

Tashbayev, B. 60

Tassery, H. 145, 153

Tedesco, T.K. 47, 54, 138

Tellez-Rojo, M. 102

Tenuta, L.M.A. 101

Terčelj, M. 80

Terrer, E. 145,153

Thonat, C. 145

Tompkins, G. 167

Toniolo, J. 58

Topolovec, L. 137

Toth, Z. 26

Toumba, K.J. 162

Tse, K.Y. 46

Türkmen, E. 15

Turton, B. 134

Ungar, P. 2

Usai, P. 40

Usuga-Vacca, M.V. 93, 121

Valadas, L.A.R. 127, 128

Valappil, S.P. 18, 36

Vale, G.C. 12

Valenzuela-Verdugo, M.J. 109

Van den Braak, M.C.T. 148 van der Veen, M.H. 71, 90

Van Empelen, P. 147, 148

Van Loveren, C. 147,148

Van Meijel, B. 147, 148

van Wijk, A.J. 59

Vannahme, C. 3

Varjão, M.M. 59

Vera-Bueno, H. 109

Vercruysse, C. 97

Verrips, G.H.W. 148

Vidmar, J. 132

Vizzotto, M.B. 118

Volgenant, C.M.C. 90

Wagner, M.B. 87

Walker, G.D. 20

Waters, E. 33

Wertheimer, A. 6

West, N. 155

Wierichs, R.J. 16

Wilde, S. 163

Witecy, C. 64

Woelber, J.P. 30

Wollgarten, S. 43, 154

Wong, F.S.L. 14

Wróbel-Dudzińska, D. 41

Wu, Y. 25

Wu, Y.F. 35

Xing, H. 156

Yang, J.M. 35

Yanikoglu, F. 15, 159

Yarat, A. 114

Yoshioka, L. 47

Young, A. 60

Yuan, Y. 20

Zabokova Bilbilova, E. 79

Zabokova-Bilbilova, E. 11, 56, 151, 165

Zaks, B. 26

Żarnowski, T. 41

Zawia, E.A. 162

Zenkner, J.E.A. 87

Zhou, X.D. 23

Ziebolz, D. 48, 95

Zohoori, F.V. 103, 115 